\title{
WestVirginiaUniversity
}

THE RESEARCH REPOSITORY @ WVU

Graduate Theses, Dissertations, and Problem Reports

2003

\section{Geological mapping of entry roof in mines}

Quanzhong Gu

West Virginia University

Follow this and additional works at: https://researchrepository.wvu.edu/etd

\section{Recommended Citation}

Gu, Quanzhong, "Geological mapping of entry roof in mines" (2003). Graduate Theses, Dissertations, and Problem Reports. 2479.

https://researchrepository.wvu.edu/etd/2479

This Dissertation is protected by copyright and/or related rights. It has been brought to you by the The Research Repository @ WVU with permission from the rights-holder(s). You are free to use this Dissertation in any way that is permitted by the copyright and related rights legislation that applies to your use. For other uses you must obtain permission from the rights-holder(s) directly, unless additional rights are indicated by a Creative Commons license in the record and/ or on the work itself. This Dissertation has been accepted for inclusion in WVU Graduate Theses, Dissertations, and Problem Reports collection by an authorized administrator of The Research Repository @ WVU.

For more information, please contact researchrepository@mail.wvu.edu. 


\title{
Geological Mapping of Entry Roof in Mines
}

\author{
Quanzhong Gu \\ Dissertation Submitted to the \\ College of Engineering and Mineral Resources \\ at West Virginia University \\ in partial fulfillment of the requirements for the degree of \\ Doctor of Philosophy \\ in \\ Mining Engineering
}

\author{
Syd S. Peng, Ph.D., Chair \\ Yi Luo, Ph.D. \\ Keith A. Heasley, Ph.D. \\ Department of Mining Engineering \\ E. James Harner, Ph.D. \\ Department of Statistics \\ Bruce Kang, Ph.D. \\ Mechanical and Aerospace Engineering \\ Morgantown, West Virginia
}

2003

Key words: Geological Mapping, Drilling Parameters, Drilling Mechanical Model, Drilling Hardness, Energy Equilibrium, Discriminant Analysis, Rock Type Classification

Copyright 2003 Quanzhong Gu 


\section{Abstract \\ Geological Mapping of Entry Roof in Mines \\ Quanzhong Gu}

Although strata types and approximate thickness of strata can be obtained from the geological exploration materials of the mine, these do not meet the requirements for roof bolting design because of the ever-changing roof geological properties, including rock strength, discontinuity distribution, and thickness of rock strata. In order to obtain such geological information in a timely manner, the technology of geological mapping of the roof in the mine, which uses the drilling parameters acquired during the drilling process, has been proposed. The study in this dissertation attempts to map the roof geology in real time by developing a new drilling parameter, drilling hardness, to detect the locations of interfaces between rock layers and discontinuities, and to classify the rock types. The derivation of the drilling hardness in this research involves the geometry of the drill bit/and contact area between the drill bit and rock, the friction between the drill bit and rock, and the energy lost in kinetic energy, potential and torsion energies.

In conjunction with the laboratory test, the drilling mechanical model (DMM) and the drilling hardness were successfully developed based on the principle of energy equilibrium. It was found that the values of the monitored drilling parameters were affected by multiple factors, such as rock properties, friction between the drill bit and the rock, energy lost and adjustment of the hydraulic system of the drilling machine. By considering the energy lost, the contact area and friction between the drill bit and rock, the derived drilling parameter, drilling hardness and its slope can be used to effectively detect the locations of interfaces between rock layers. Also, the criteria for using the slope to determine whether it is an interface or a discontinuity were developed. On average, the errors in interface location between the predicted ones and these shown by borehole camera scope and actual core logs are 1.37 inches at Mine A and 1.22 inches at Mine B, respectively. Although the accuracy in determining the locations of discontinuities within one rock layer is not conclusive, the analysis results from the Mine A and Mine B show that the error for the discontinuity location identification is very small, and the average error is less than 1.481 inches. 
In addition, the algorithm for classifying/predicting rock type using discriminant analysis was also determined. However, the requirement of obtaining a core log first in order to build the training data for each rock type and updating the group data if roof geology changes significantly limits the applicability of this technology and makes it unfeasible in applying this discriminant analysis in the field. 


\section{Acknowledgements}

I would like to express my sincere appreciation to those who have contributed to the preparation and completion of this dissertation. My deepest thanks to those who helped, supported, and encouraged me over the duration of my Ph.D studies. With respect and gratitude, I am deeply indebted to all members of my advisory and examination committee: Dr. Syd. Peng, Dr. E. James Harner, Dr. Keith A. Heasley, Dr. Yi Luo, and Dr. Bruce Kang for their supports and invaluable suggestions during the research work.

I like to thank my fellow students and staff of College of Engineering and Mineral Resource for their help. I will never forget the discussions and debates made with those who shared the same interests in some topics, such as Roof Bolting, Ground Control, Neural Network, Multivariate Analysis, and Pattern Classification.

I also would like to thank Gene Wilson and Craig Collins from J.H. Fletcher Inc. and Ben Mirabile and Jisheng Han from the Dept. of Mining Engineering, WVU, for their field testing work.

I sincerely appreciate the support from the U.S. Dept. of Energy Industry of the future (Mining Sector) Program under contract No. DE-FC26-01NT41056 and Coal and Energy Research Bureau (CERB), State of West Virginia.

I sincerely thank my family especially my wife Shuhong $\mathrm{Xu}$ for ker suggestions and patience, my parents, my brother, and my sister for their limitless support, encouragement, and believing in me through my life to aim high and achieve success.

I am dedicating this work to my son, Evan Gu. 


\section{Table of Contents}

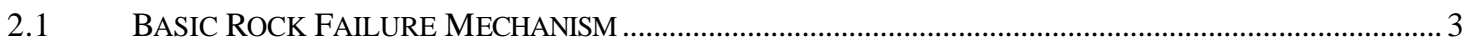

2.2 FREQUENTLY DRILLED STRATA ASSOCIATED WITH COAL SEAMS IN THE US ................................... 3

2.3 PARAMETERS COMMONLY USED IN SPECIFYING DRILLING............................................................

2.4 TYPICAL REAL-TIME DATA ACQUISITION SYSTEM FOR ROCK BOLT DRILLING............................... 9

2.4.1 System Developed by Parvus Corporation ${ }^{[3]}$........................................................................ 9

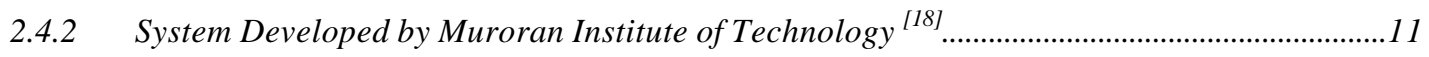

2.4.3 Feedback Control System Developed by Structured Mining System, Inc., J. H. Fletcher \&

Company and West Virginia University..........................................................................................12

$2.5 \quad$ ANALYSIS OF DRILLING PARAMETERS IN THE PAST .................................................................... 14

2.5.1 The Analysis of Drilling Parameters Implemented by Spokane Research Center...................21

2.5.2 Drilling Parameters Implemented by Itakura and his Colleagues ${ }^{[14][15][16] \ldots \ldots \ldots \ldots \ldots \ldots \ldots \ldots \ldots \ldots \ldots . . .25}$

2.5.3 Preliminary Analysis of Drilling Parameters Implemented by West Virginia University......29

2.5.3.1 Variations in Drilling Parameters While Drilling.................................................................. 32

2.5.3.2 Relationship between Specific Energy and Rock Strength ..................................................... 37

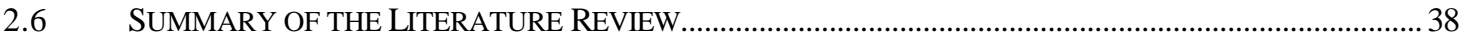

3 RESEARCH OBJECTIVES AND SCOPE AND METHODOLOGY ....................................................40

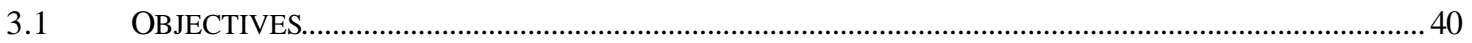

3.2 SCOPE OF RESEARCH .............................................................................................................. 40

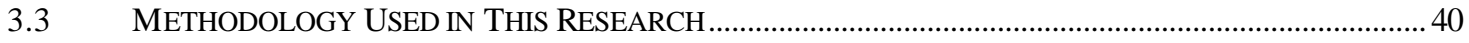

3.3.1 Data Filtering and Data Smoothing ……………................................................................

3.3.2 Determination of Rock Properties......................................................................................

3.4 DATA FILTER AND SMOOTHING AND SAMPLING....................................................................... 41

3.4.1 Filtering of Data Sets Sharing the Same Position ………………………………………….........43

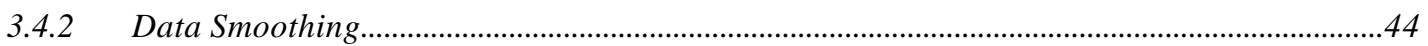

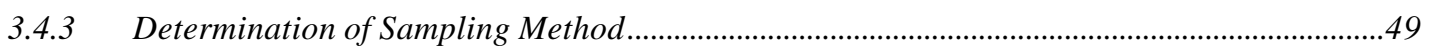

3.4.4 Summary of Data Filter and Data Smoothing …………………………….............................52

4 DEVELOPMENT OF ALGORITHMS FOR DETERMINATION OF ROCK PROPERTIES 53

4.1 DETERMINATION OF A NEW DRILLING PARAMETER - DRILLING HARDNESS (DH) ........................56

4.1.1 Determination of the Frictional Coefficient ………………………………………………........

4.1.2 Determination of Contact Area in Axial Direction ……………………………........................5 
4.1.3 Drilling Mechanical Model (DMM) and Determination of the Drilling Hardness.................59

4.2 DETERMINATION OF THE LOCATIONS OF INTERFACES AND DISCONTINUITIES..............................63

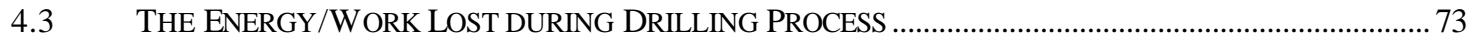

4.3.1 Energy Lost in Rotational Direction.....................................................................................

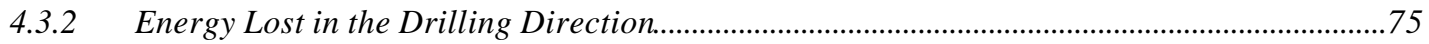

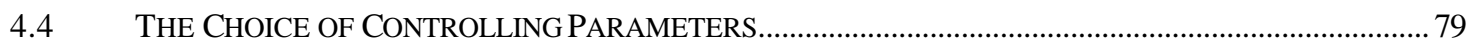

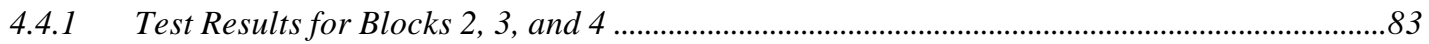

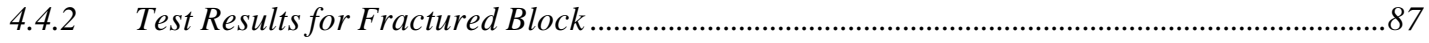

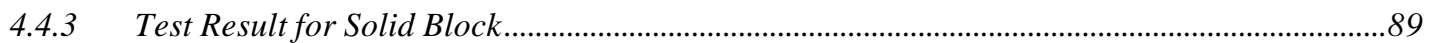

4.4.4 Test Result for Block 6 (Layered Block)....................................................................................89

4.4.5 Summary of the Influences of the Pre-settings before Drilling ..................................................89

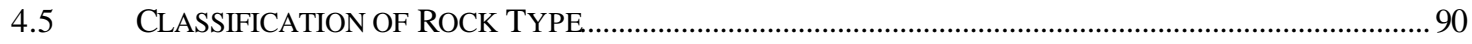

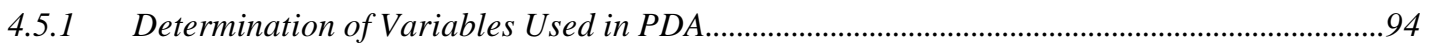

4.5.2 Determination of Group Data and Covariance Matrix in PDA...............................................99

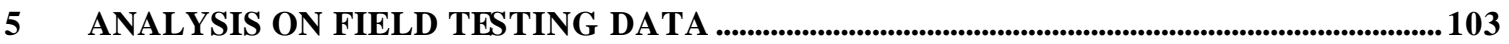

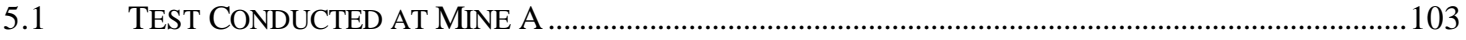

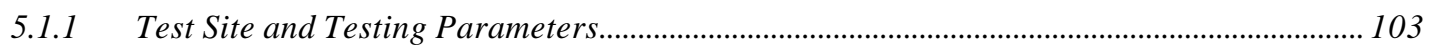

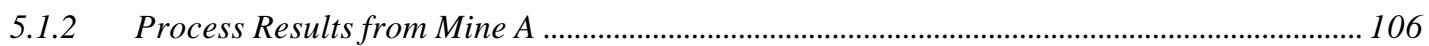

5.1.3 Rock Type Classification Using Supervised Discriminant Analysis................................... 114

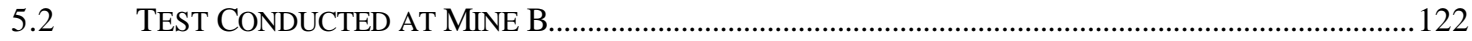

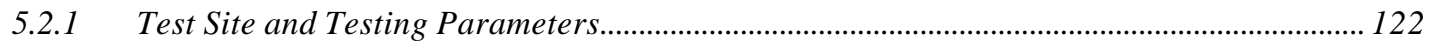

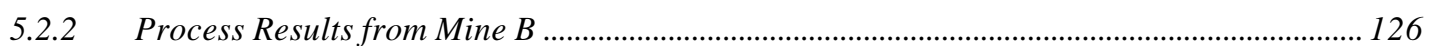

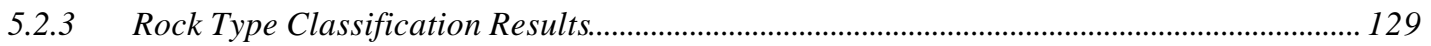

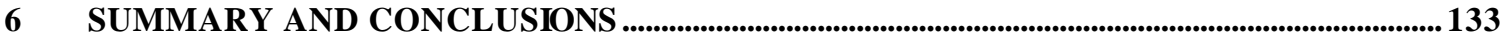

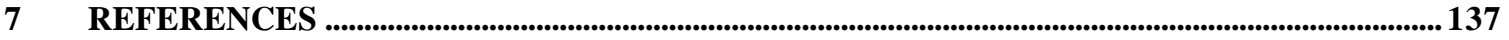

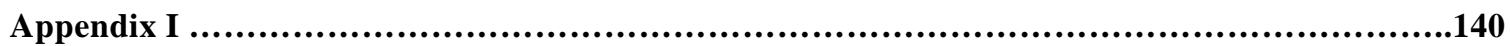

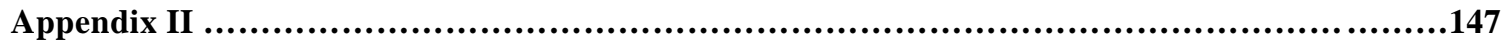

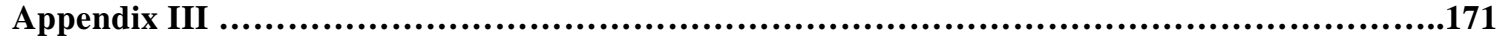

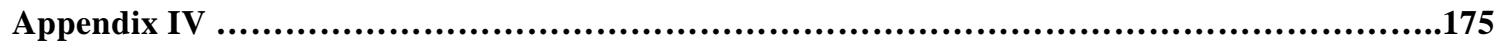




\section{List of Figures}

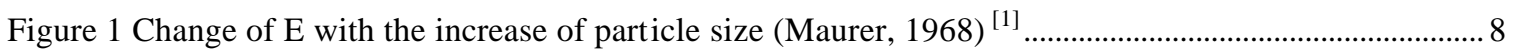

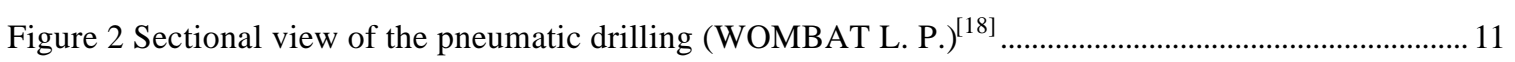

Figure 3 J.H. Fletcher's Bolter and Feedback Control Station ${ }^{[21]}$................................................................ 13

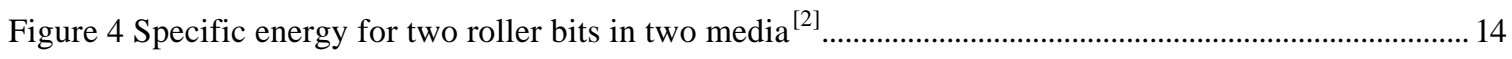

Figure 5 Specific energy and penetration per revolution for percussive-rotary and rotary drilling in Pennant

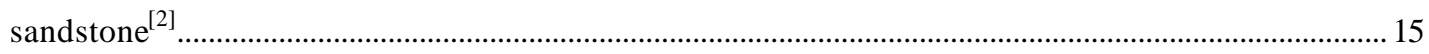

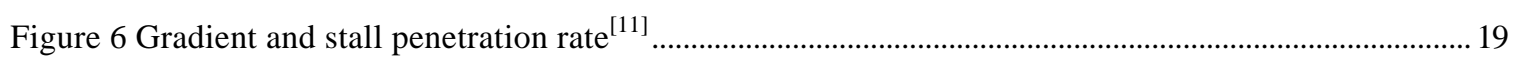

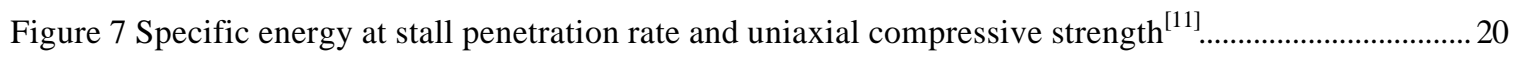

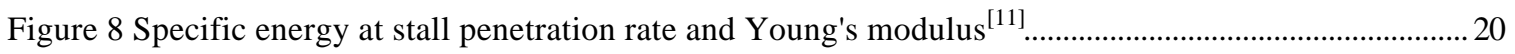

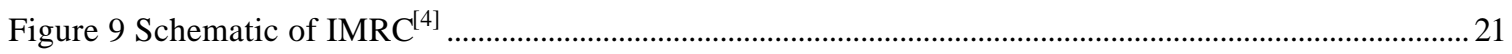

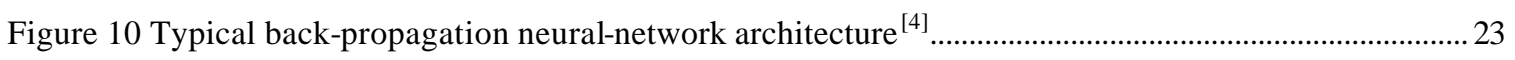

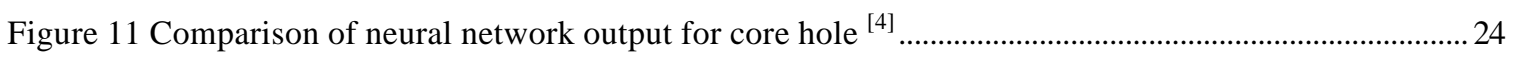

Figure 12 Theoretical patterns of mechanical data for various types of discontinuities ${ }^{[16]}$............................ 26

Figure 13 Simplified variation learning patterns of discontinuities for back-propagation neural network ${ }^{[16]}$

……

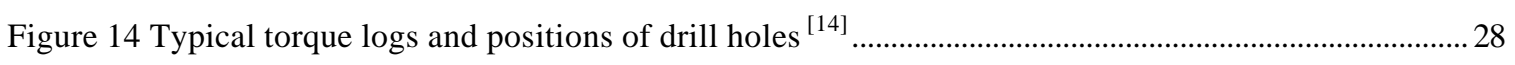

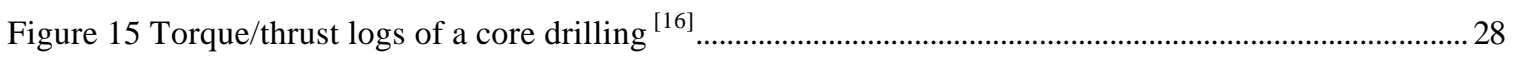

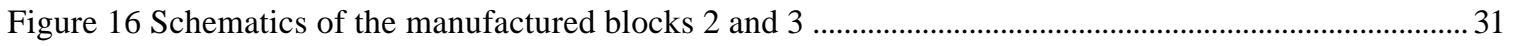

Figure 17 Schematics of the manufactured rock layer block 4 and block 5 with fractures .............................. 32

Figure 18 An example of the drilling parameters for manufactured block with rock layers ${ }^{[10]}$....................... 33

Figure 19 An example of the drilling parameters for manufactured blocks with simulated fractures ${ }^{[10]} \ldots . . .35$

Figure 20 Correlation between SED and UCS of rock layers in blocks 2, 3, and 4......................................... 38

Figure 21 An example of the data sets without filtering (under stall condition) ................................................ 42

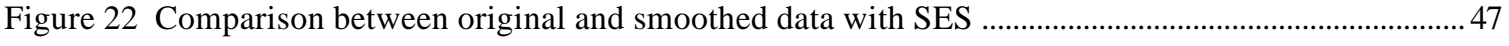

Figure 23 Comparison between original and smoothed data with DES .............................................................. 47

Figure 24 Comparison between original and smoothed data with NWS .......................................................... 48

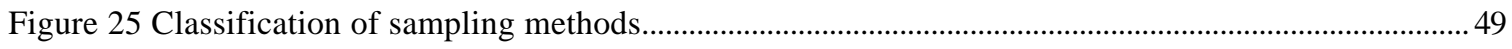

Figure 26 Comparison between SD (smoothed data) and SSD (smoothed and sampled data), .......................51

Figure 27 The layered block (Block 6) drilled in laboratory ............................................................................5

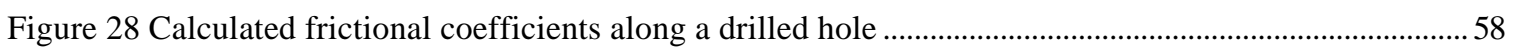

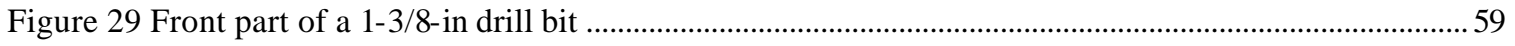

Figure 30 Schematic of the force components in drilling operation ................................................................ 60

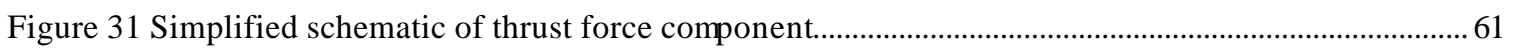

Figure 32 An example of the drilling hardness (Hole 4, Block 6) .................................................................... 62

Figure 33 An example of slope distribution along a drilled hole (Hole 4, Block 6) ...........................................66 
Figure 34 Drilling hardness and its slope distribution along a drill hole (Hole 1, Block 6) 69

Figure 35 Drilling hardness and its slope distribution along a drill hole (Hole 2, Block 6) ............................70

Figure 36 Drilling hardness and its slope distribution along a drill hole (Hole 3, Block 6) ........................... 70

Figure 37 Drilling hardness and its slope distribution along a drill hole (Hole 5, Block 6) ........................... 71

Figure 38 Drilling hardness and its slope distribution along a drill hole (Hole 6, Block 6) ............................ 71

Figure 39 Drilling hardness and its slope distribution along a drill hole (Hole 7, Block 6) .............................72

Figure 40 Drilling hardness and its slope distribution along a drill hole (Hole 8, Block 6) ...........................72

Figure 41 An example of energy variation in the rotational direction (Hole 4, Blcok 6) .............................. 74

Figure 42 Percentage of torsion energy in the work done by torque for all 8 holes in Block $6 \ldots \ldots \ldots \ldots \ldots \ldots \ldots . . . . . . .74$

Figure 43 Percentage of kinetic energy in the work done by torque for all 8 holes in Block $6 \ldots \ldots \ldots \ldots \ldots \ldots \ldots . . . . . . .75$

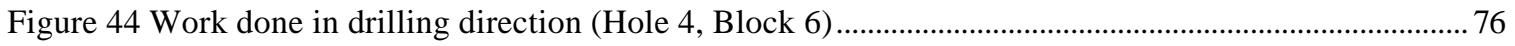

Figure 45 Percentage of the total energy lost in the work done by thrust for all 8 holes in Block $6 \ldots \ldots \ldots \ldots . . . . .77$

Figure 46 Percentage of kinetic energy in the work done by thrust for all 8 holes in Block 6 ..................... 77

Figure 47 Percentage of potential energy in the work done by thrust for all 8 holes in Block $6 \ldots \ldots \ldots \ldots \ldots \ldots . . . . .77$

Figure 48 Percentage of work done to overcome friction in the work done by thrust for all 8 holes in Block

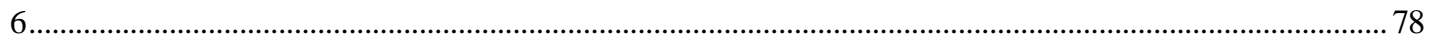

Figure 49 Ideal transition of a drilling parameter near the interface between rock layers ............................. 80

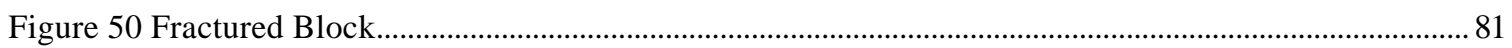

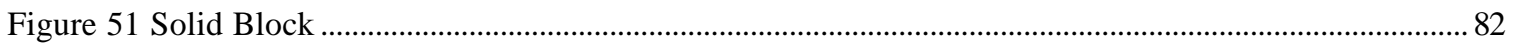

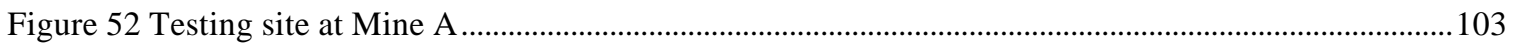

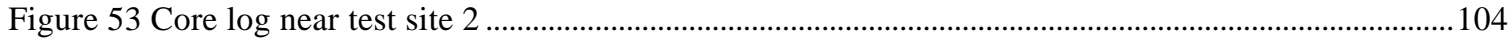

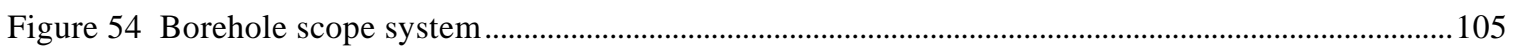

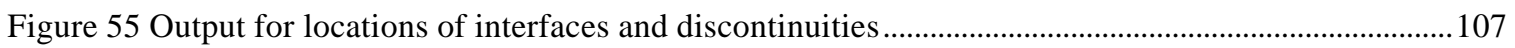

Figure 56 Drilling hardness and slope for Hole 13, Mine A .....................................................................108

Figure 57 Drilling hardness and slope for Hole 14, Mine A ......................................................................108

Figure 58 Drilling hardness and slope for Hole 15, Mine A ........................................................................109

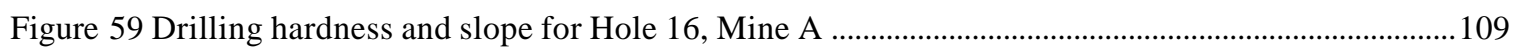

Figure 60 Drilling hardness and slope for Hole 17, Mine A …..................................................................110

Figure 61 Drilling hardness and slope for Hole 18, Mine A ......................................................................110

Figure 62 Drilling hardness and slope for Hole 19, Mine A .......................................................................111

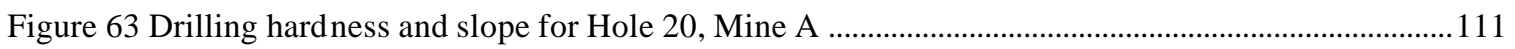

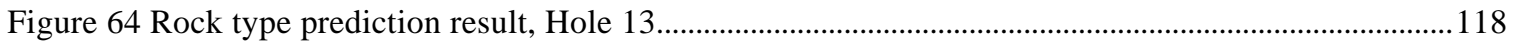

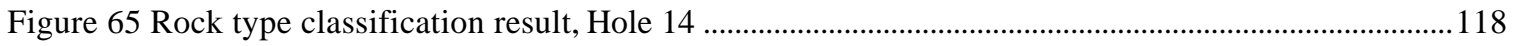

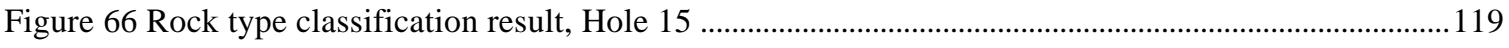

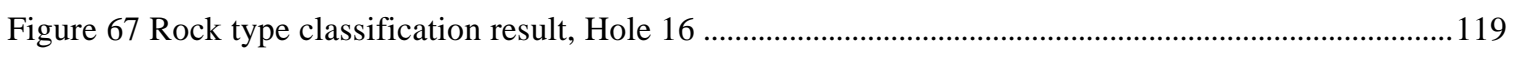

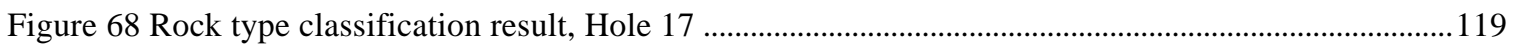

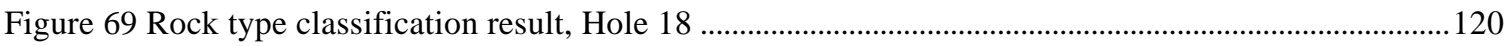


Figure 70 Rock type classification result, Hole 19 120

Figure 71 Rock type prediction result, Hole 20 .. 120

Figure 72 Testing sites at Mine B .. .123

Figure 73 Core log in Area-1 and Area-2L

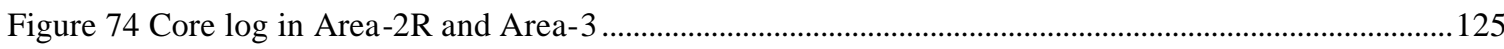

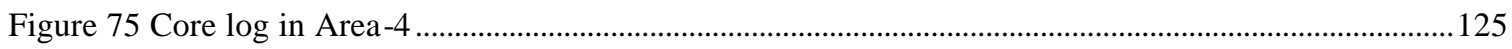

Figure 76 Comparison of the interface location in different areas .............................................................. 128 


\section{List of Tables}

Table 1 Typical Specific Energy Requirements for Conventional Crushing (Joules/ $\mathrm{cm}^{3}$ ) $\left(\right.$ Maurer, 1968) ${ }^{[1]} .7$

Table 2 Calculated specific energy $\mathrm{SE}_{\mathrm{v}}(\mathrm{T})$ and predicted specific energy $\mathrm{SE}_{\mathrm{v}}(\mathrm{T})$ from strength properties ${ }^{[12]}$

Table 3 Calculated specific energy $\mathrm{SE}_{\mathrm{v}}(\mathrm{w})$ and predicted specific energy $\mathrm{SE}_{\mathrm{v}}(w)$ from strength

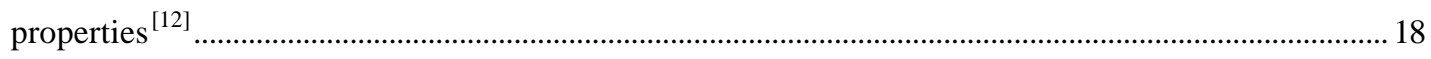

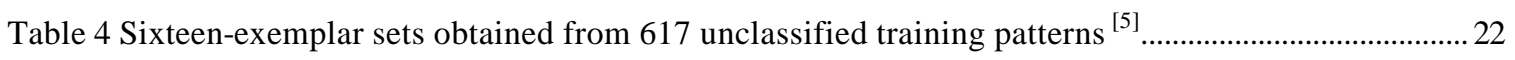

Table 5 A verage rock mechanics properties of rock units in the test blocks ${ }^{[10]}$.............................................. 29

Table 6 Variation trends of drilling parameters while drilling in manufactured blocks with embedded rock

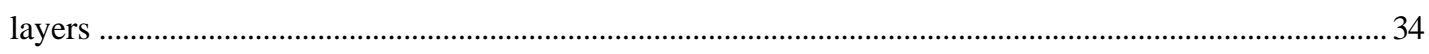

Table 7 Variation trends of drilling parameters while drilling in manufactured blocks with simulated

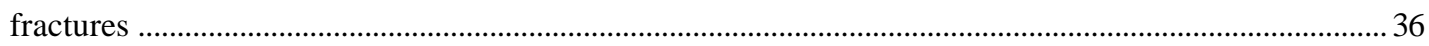

Table 8 Range of specific energy for rock layers in blocks 2 - 4 ..................................................................... 37

Table 9 An example of the comparison with different data smoothing methods (thrust)................................. 46

Table 10 The average number of recorded data sets within one-inch horizon of rock layers, Block 6

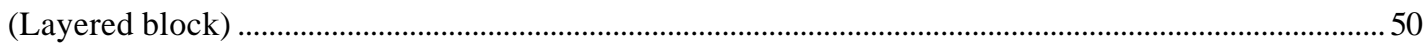

Table 11 Comparison of standard deviation before and after sampling (thrust, for smoothed data), Block 6

Table 12 Reduction percentage of data sets after data filtering and smoothing and sampling, Block 6 ........ 52

Table 13 Parameters used in laboratory testing on Block 6 ............................................................................5

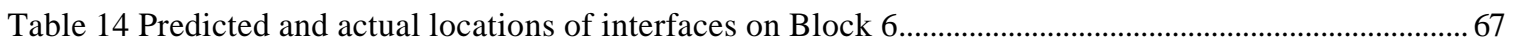

Table 15 Laboratory testing control parameters for Fractured Block................................................................ 82

Table 16 Laboratory testing control parameters for Solid Block ...................................................................... 83

Table 17 Predicted and actual locations of interfaces on Block 2.................................................................. 84

Table 18 Predicted and actual locations of interfaces on Block 3 ………………………............................. 85

Table 19 Predicted and actual locations of interfaces on Block 4................................................................. 86

Table 20 Predicted and actual locations of fractures on Fractured Block.......................................................... 88

Table 21 Presumed choices of variable determination ..................................................................................... 95

Table 22 Comparison of classification and/or prediction with the five variable-selections .............................. 97

Table 23 Hit rate comparison among three rock types with the five variable-selections.................................. 98

Table 24 Comparison of the prediction results using different covariance matrixes .........................................102

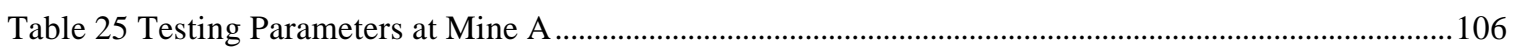

Table 26 Locations of interfaces and discontinuities determined from the field data using the scope data and

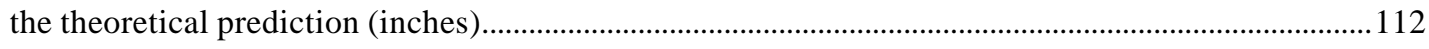

Table 27 An example of rock classification (Hole 17, Mine A).....................................................................115 
Table 28 Rock type classification/prediction results from Mine A (Holes 13 to 20) ....................................121

Table 29 Hit rates of the predicted and classified rock types............................................................................. 122

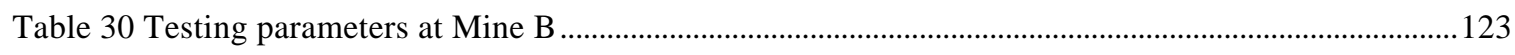

Table 31 Locations of interfaces and discontinuities determined from the field data using the core log and

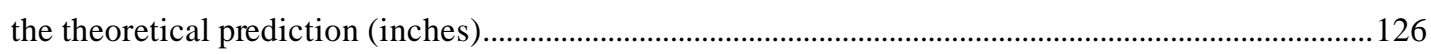

Table 32 Hit rates of the predicted and classified rock types (Group data obtained from Area-4) ..............130

Table 33 Hit rates of the predicted and classified rock types (Group data obtained from Area-1, 2, 3 and 4) 


\section{Introduction}

\section{Introduction}

Rock bolts have been the most dominant type of support in underground construction since the 1980s because of their easy installation, light weight, convenient transportation and relatively high anchorage capacity. Bolts can be employed to solve ground control problems that involve direct tension, sliding, and dynamic load. Because of the advantages of using rock bolting, more and more countries are actively developing the rock bolting system for entry support.

Variation in rock properties between mines complicates rock bolting design and the application of bolt-based support systems. Choosing a bolt type and designing bolting parameters that are economical and meet the support needs of ever-changing geological conditions and various support requirements is a difficult task and a real challenge for mining engineers. Regardless of the bolting design method used, the major factors that the designer needs to be concerned are strata types, uniaxial compressive strength (UCS) of the strata, thickness of the strata, and distribution of joints or fractures.

In the field, geological conditions vary considerably even within the same rock layer; the thickness and locations of layer interfaces, fracture distribution and rock strength change from one location to the next. If the roof condition suddenly deteriorates and the bolting design is not adjusted, accidents related to roof falls might consequently happen. In order for roof bolts to perform reliable, detailed information about geological and geomechanical conditions around the entry must be determined, such as the interfaces or separations of rock layers, especially the positions and thickness of weak planes, and thickness variation in the roof strata.

In an effort to determine rock properties of the entry roof in real time by using drilling parameters such as thrust, penetration rate, torque, rotation rate, and bit position, and to make roof bolting design meet the needs of ever-changing geological conditions, 


\section{Introduction}

extensive research has been conducted. Based on laboratory tests, Teale ${ }^{[2]}$ found that the minimum specific energy matches the corresponding rock's uniaxial compressive strength. This relationship is so profound that it has guided researchers to try to use those mechanical drilling parameters to reflect rock properties. In the early 1990's, Itakura, et $\mathrm{al}^{[14][15][16]}$, studied the use of recorded drilling parameters and developed a methodology to determine the locations of bed separation and fractures. Since June 1999, West Virginia University has been cooperating with J. H. Fletcher \& Company in the development and application of the roof bolter feedback system. The feedback system is a real-time data logging system, which can directly monitor and control the drilling parameters such as thrust, torque, rotation rate (Revolutions per minute), and bit position while drilling.

The major objective of this work is to develop a methodology and algorithm that uses drilling parameters to define and map the entry roof's geological properties such as the locations of interfaces between rock layers and discontinuities within the same rock layer; thus improve the understanding and prognosis of the geological structures. 


\section{$\underline{2 \text { Literature Review }}$}

\section{Literature Review}

\subsection{Basic Rock Failure Mechanism}

Rock drilling can be achieved by one of four basic mechanisms ${ }^{[1]}$ : (1) mechanically induced stress by impact, abrasion, and erosion, (2) thermally induced stress created by heat, (3) fusion and vaporization, and (4) chemical reactions.

The latter three mechanisms are not typically used in drilling a hole for roof bolting. Universally the first mechanism, mechanically-induced stress, is used with the roof bolter. Drills produce mechanical stresses induced by impact and abrasion. When these stresses exceed a rock's tensile or shear strength, brittle fracture and/or plastic yielding takes place ${ }^{[1]}$. During the drilling process, thrust continuously pushes the bit into rock, while torque continuously gives the bit a rotary movement to break out fragments. When thrust is applied on a rock surface, a crushed zone or finely powdered rock, is formed beneath the bit. Meanwhile, toque makes the bit abrade and break the fragments out.

\subsection{Frequently Drilled Strata Associated with Coal Seams in the US}

Rock types may vary with different regions. For instance, a frequent succession of strata found in the coal-bearing formation of the Appalachian region is (from floor to roof): sandstone, limestone, clay, coal, shale and sandstone ${ }^{[13]}$. However, in a specific coal mine in the Pittsburgh coal seam, the succession of strata from floor to roof is: shale, sandstone, coal, shale with sandstone, shale, siltstone ${ }^{[13]}$. Although there is some difference of strata associated with different coal-beds, several types of sedimentary strata are very common, such as shale and sandstone. Roof strata commonly associated with coal seams include shale, claystone, siltstone, sandstone, limestone, and mudstone. 


\section{$\underline{2 \text { Literature Review }}$}

\subsection{Parameters Commonly Used in Specifying Drilling}

There are three typical methods of mechanical drilling: rotary, percussive, and rotarypercussive. Rotary is the commonly-used drilling method of the roof bolter in the coal industry. For this drilling method, drilling parameters can be grouped into two classes, original drilling parameters and derived drilling parameters. The original drilling parameters are directly monitored and recorded from the data acquisition system, while the derived parameters are derived from the original parameters based on certain relationships. The original drilling parameters include: thrust, torque, rotation rate, penetration rate, and bit position. The derived drilling parameters include specific energy, penetration per revolution, horsepower, angular velocity and angular acceleration, etc.

The original drilling parameters can be defined as follows:

(1) Thrust (lbs). Produced by motor and applied on the drilling rod \& bit. The bit could be continuously pushed into the rock and induce initial compressive folia of the rock to be drilled.

(2) Torque (in-lbs). Produced by motor and applied on the drilling rod \& bit. The bit is given a lateral/rotary movement to break out fragments of rock.

(3) Rotation rate (rev/min). Revolutions of drilling rod per minute.

(4) Penetration rate $(\mathrm{in} / \mathrm{sec})$. Distance that a drilling bit penetrates into rock per second. Usually, under a certain thrust, the penetration rate increases with torque $^{[2]}$.

(5) Bit position (in). It is the distance from the hole-mouth to where the bit is while drilling. 


\section{Literature Review}

The derived drilling parameters can be defined as follows:

(1) Penetration per revolution, ppr. It is the ratio of penetration rate to rotation rate.

$$
p p r=\frac{60 \cdot u}{N}, \mathrm{in} / \mathrm{rev}
$$

where $\mathrm{u}$ - penetration rate, $\mathrm{in} / \mathrm{sec}$.

$$
\mathrm{N} \text { - rotation speed, RPM }
$$

(2) Angular velocity, $\omega$. Rate of change of its angular position, rad/sec

$$
\omega=\frac{N \cdot 2 \pi}{60}, \mathrm{rad} / \mathrm{sec}
$$

(3) Angular/rotation acceleration, a. Rate of change in angular velocity, $\mathrm{rad} / \mathrm{sec}^{2}$

$$
a_{i}=\frac{\omega_{i}-\omega_{i-1}}{t_{i}}, \mathrm{rad} / \mathrm{sec}^{2}
$$

where $a_{i}-$ rotation acceleration at time $i$;

$$
t_{i}-\text { time spent from } \omega_{i-1} \text { to } \omega_{i} \text {. }
$$

(4) Work, w: Work is the product of an applied force and the distance through which it moves the body to which it is applied. In rotary drilling

$$
w=F \cdot u+T \cdot \theta=F \cdot u+\frac{2 \pi \cdot N \cdot T}{60}, i n-l b / \mathrm{sec}
$$

where F - thrust, lbs

$\mathrm{T}$ - torque, in-lbs 


\section{Literature Review}

$\theta$ - angular distance, $2 \pi \times$ rotation rate $/ 60$.

In Eq. 4, the term $F \cdot u$ is the work done by thrust, and the term $\frac{2 \pi \cdot N \cdot T}{60}$ is the work done by torque.

(5) Horsepower. Power is the rate at which work is done. In other words, power is the amount of work done in one minute or one second. Usually, the unit of power is horsepower, which is the work at the rate of $550 \mathrm{ft}-\mathrm{lbs} / \mathrm{sec}$ or $6600 \mathrm{in}-\mathrm{lbs} / \mathrm{sec}$ :

$$
H P=\frac{\text { power }}{550}=\frac{1}{550} \cdot \frac{\text { Work or Energy in } \mathrm{ft}-\mathrm{lb}}{\sec \text { onds }}=\frac{\text { Work or Energy in in }-\mathrm{lb}}{6600 * \sec \text { onds }}
$$

In some rotary drilling systems, such as the roof bolter developed by J. H. Fletcher \& Company, the power supplied by the hydraulic system can be divided into two parts. One is for torque, and the other is for thrust. The actual output power of the drilling hydraulic system is the sum of power for torque and thrust. The power distributed to torque and thrust can be calculated from the following equations:

$$
\begin{aligned}
& H P_{-} \text {thrust }=\frac{F \cdot u}{6600} \\
& H P_{-} \text {torque }=\frac{T \cdot \theta}{6600}=\frac{T * 2 \pi * N}{6600 * 60}
\end{aligned}
$$

The total power for drilling is then:

$$
\mathrm{HP}=\mathrm{HP}_{-} \text {thrust }+\mathrm{HP} \_ \text {torque }
$$

(6) Specific Energy of drilling, SE. Specific energy of drilling is defined as the energy required to excavate one unit volume of rock per unit time. According to Teale ${ }^{[2]}$, as the efficiency of the drilling operation increases (when appropriate thrust and rotational 


\section{Literature Review}

speeds are reached) the specific energy decreases until it approaches, or is roughly equal to, the compressive strength of the rock being drilled.

In the past, the specific energy was expressed by Maurer ${ }^{[1]}$ :

$$
E=10 E_{i}\left(\frac{1}{\sqrt{p}}-\frac{1}{\sqrt{f}}\right), \text { Joules } / \mathrm{cm}^{3}
$$

where E - specific energy

$$
\begin{aligned}
& \mathrm{E}_{\mathrm{i}} \text { - specific energy required to crush rock from infinite size to } \\
& \quad 0.1 \mathrm{~mm}, \text { Joules } / \mathrm{cm}^{3} \\
& \text { p - final particle size, microns } \\
& \text { f - initial particle size, microns }
\end{aligned}
$$

Table 1 and Figure 1 give typical values of specific energy for crushing various rocks.

Table 1 Typical Specific Energy Requirements for Conventional Crushing $\left(\right.$ Joules $\left./ \mathrm{cm}^{3}\right)\left(\right.$ Maurer, 1968) ${ }^{[1]}$

\begin{tabular}{|c|c|c|c|}
\hline \multirow{2}{*}{ Rock } & \multicolumn{3}{|c|}{ Crushed particle size } \\
\cline { 2 - 4 } & $0.1 \mathrm{~mm}$ & $1 \mathrm{~mm}$ & $10 \mathrm{~mm}$ \\
\hline Glass & 30 & 10 & 3 \\
\hline Sandstone & 110 & 35 & 11 \\
\hline Limestone & 110 & 35 & 11 \\
\hline Dolomite & 110 & 35 & 11 \\
\hline Quartzite & 120 & 38 & 12 \\
\hline Quartz & 120 & 38 & 14 \\
\hline Granite & 140 & 45 & 15 \\
\hline Shale & 150 & 48 & 18 \\
\hline Taconite & 180 & 57 & 21 \\
\hline Basalt & 210 & 67 & \\
\hline
\end{tabular}




\section{$\underline{2 \text { Literature Review }}$}

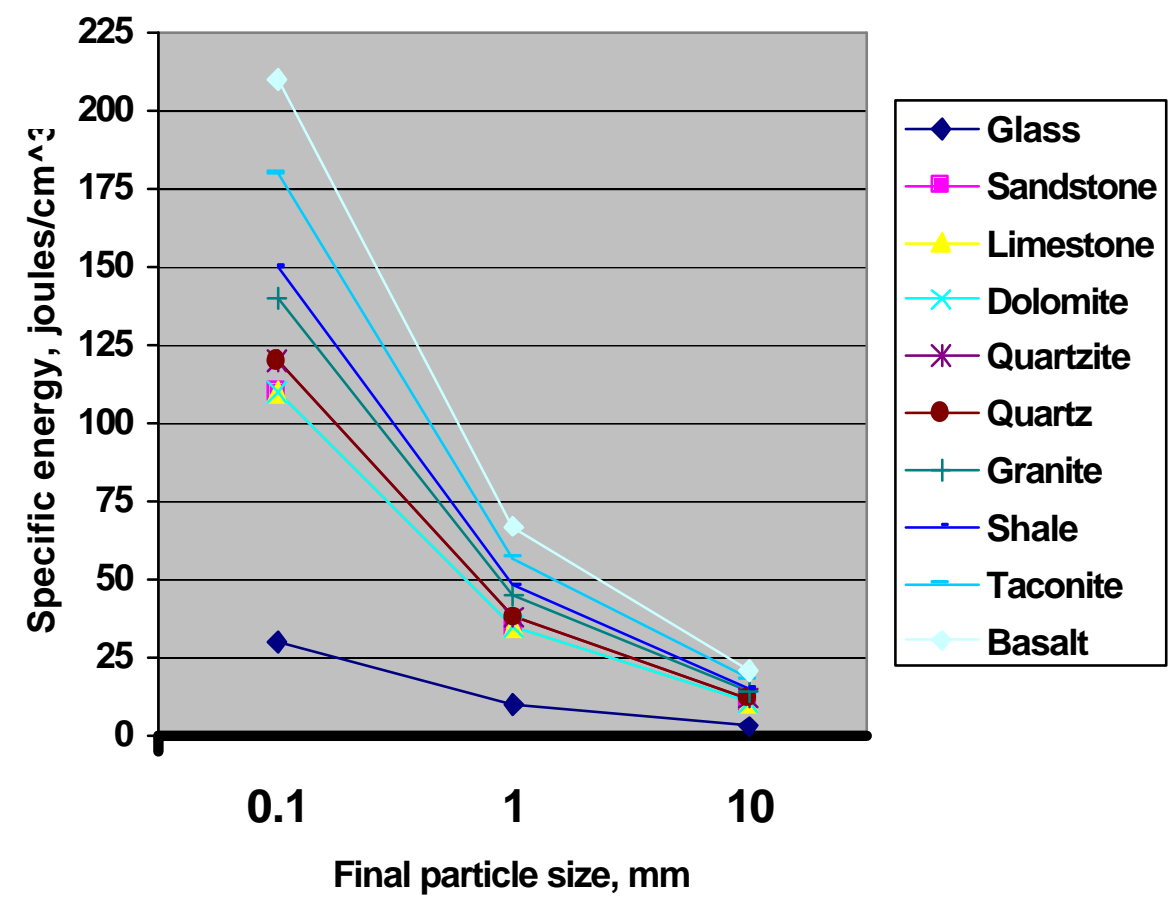

Figure 1 Change of $\mathrm{E}$ with the increase of particle size (Maurer, 1968) ${ }^{[1]}$

It can be seen from Table 1 and Figure 1 that the required specific energy becomes smaller with the increase of crushed particle size, but the rate of reduction correspondingly gets smaller. There has been no specific technology to determine particle size while drilling, which leads to the difficulty in calculating specific energy with Eq. 9 in real time.

From Teale's definition of specific energy and the drilling parameters that can be measured, specific energy of drilling can be derived from Eq.4. With right items in Eq.4 divided by A.u (unit volume of rock), the specific energy can be expressed as

$$
S E=\frac{F}{A}+\frac{2 \pi \cdot N \cdot T}{A \cdot u \cdot 60}, \mathrm{psi}
$$

where A - cross-sectional area of the drilled hole, in ${ }^{2}$. 


\section{Literature Review}

The specific energy required to excavate one unit volume of rock varies among different strata types because all the drilling parameters change with the variations of

rock types. The specific energy of drilling is influenced by a number of parameters ${ }^{[11]}$, such as sharpness of the drill bit, rock strength, rock stiffness, presence of structural discontinuities, abrasivity and hardness of the mineral constituents, nature of the rock matrix, mineral grain, and the adjustment of the hydraulic system. Theoretically, specific energy could be a potential index to determine other rock properties. However, it is influenced by so many factors that are not considered in its calculation, such as the sharpness of the drill bit and the adjustment of the hydraulic system.

\subsection{Typical Real-Time Data Acquisition System for Rock Bolt Drilling}

Drilling is a process that transfers mechanical energy in the form of thrust and/or torque to overcome resistance from rock. Development of a real-time data acquisition system is not only the first step but also a very important component in mapping geological properties of entry roof.

From the literatures, it appears that there have been mainly three realtime data acquisition systems used in rock bolting drilling in the coal industry. One was developed by Parvus Corporation (USA) in $1990^{[4]}$. The second one was developed by Muroran Institute of Technology (Japan) in $1993^{[15]}$, and the third one is a feedback control system, which has been under development since June 1999 by J. H. Fletcher \& Company and West Virginia University ${ }^{[7]}$.

\subsubsection{System Developed by Parvus Corporation ${ }^{[3]}$}

This system is a data acquisition and control (DAC) system. The hydraulic system for the model drill was designed by Rory McLaren and Associates, built by Fluidics, instrumented by the Parvus Corporation, and used by USBM, Spokane research center. 


\section{Literature Review}

The model drill has the capability of both manual and computer controls. Two proportional flow control valves were used to control the thrust and rotation of the drill. A directional flow control valve allowed the pull-down cylinder to be raised and lowered. The system was equipped with emergency shutdown and manual override capabilities with handles for manual control of rotation and thrust. The following parameters could be monitored: thrust, torque, rotation rate, penetration rate, and bit position in a drilled hole.

In this system, the hydraulic pressure differential across the motor is used to calculate drill torque. The torque applied to the drill head is calculated differentially across the input and output of the hydraulic spin unit using model EA PSIG pressure transducers manufactured by Data Instruments, Inc. The pressure transducers produce a proportional output voltage that is scaled to a psig (pounds per square inch, gauge) reading. By subtracting the output from the input pressure and multiplying by the flow rate $\left(\mathrm{ft}^{3} / \mathrm{sec}\right)$, the mechanical power $\mathrm{P}$ in $\mathrm{ft}-\mathrm{lb} / \mathrm{sec}$ can be obtained. Flow rate is derived from the pressure differentials and known constants. Torque can be obtained from the following expression: ${ }^{[3]}$

$$
T=\frac{P \cdot 30}{R P M \cdot \pi}
$$

where $\mathrm{T}$ - torque applied to drill bit, ft-lb

$\mathrm{P}$ - mechanical power, $\mathrm{ft}-\mathrm{lb} / \mathrm{sec}$

rotation rate - revolution per minute

$\pi-3.14$

The hydraulic pressure differential across the pull-down cylinder is used to calculate the drill thrust. The thrust of the drill head is measured using the same type of pressure transducers used for torque. By subtracting the output from the input, the upward thrust of the drill bit can be determined. 


\section{Literature Review}

Two valve driver modules control rotation rate (rotation rate) and thrust on the drill bit. Currently, commands from the PC operator can hold these parameters constant. For example, the proportional flow control valves can be controlled by feedback using an rotation rate sensor to maintain constant rotation rate, or by a thrust sensor to maintain constant thrust for the system.

In brief, this data acquisition and control (DAC) system has the following functions:

(1) It can monitor rotation rate of the drill bit, height of the drill head (bit position), and the hydraulic pressure used to rotate (torque) and raise the drill head (thrust).

(2) The rotation rate and thrust can be controlled and held constant. Torque and penetration rate would change in terms of the variation of rotation rate and/or thrust. Every two milliseconds, the machine checks the control node one time.

\subsubsection{System Developed by Muroran Institute of Technology ${ }^{[18]}$}

The measurement while drilling (MWD) system was developed in 1993 for smalldiameter drilling. It can monitor mechanical data (machine torque, thrust, revolution and stroke) of the drilling machine, WOMBAT L.P. Figure 2 shows the sensor arrangement for the roofbolter.

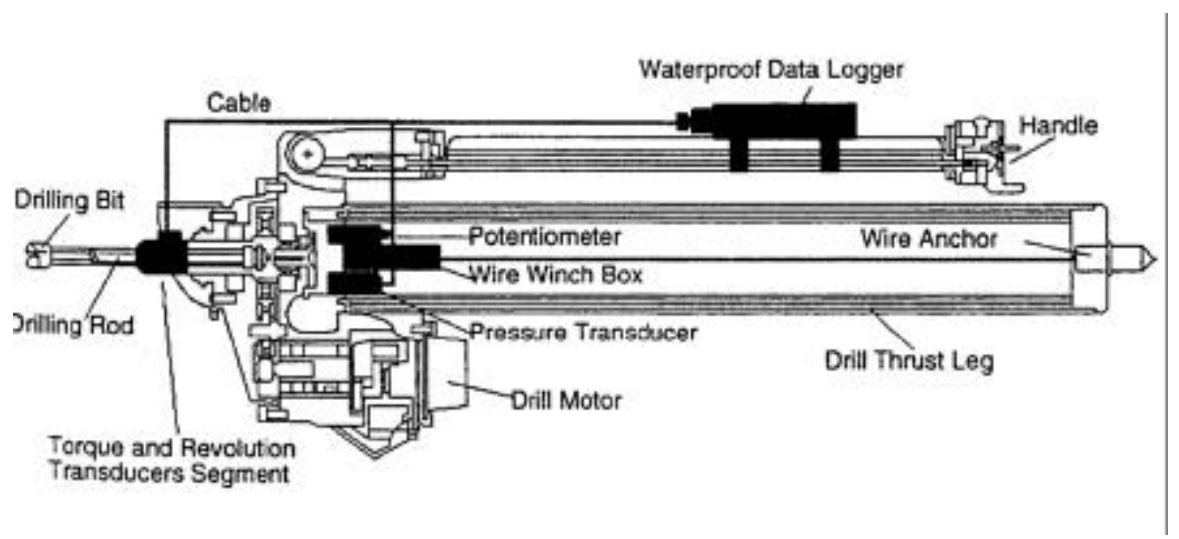

Figure 2 Sectional view of the pneumatic drilling (WOMBAT L. P.) ${ }^{[18]}$ 


\section{Literature Review}

Figure 2 shows a potentiometer and wire displacement transducer attached to the leg for stroke detection. A pressure transducer was also attached to the machine body to detect thrust. Strain gauges and a proximity switch, installed into the segment between the drilling rod and chuck, are used to detect the torque and the revolution rate of the machine, respectively. Electromagnetic coupling transmits the power of the bridge circuit and the strain data, and is proportional to the torque. All data are recorded on a removable memory card installed in a digital data logger set in the machine handle.

Briefly, the MWD system has the following capabilities:

(1) It can monitor penetration rate, rotation rate, torque, thrust and drilling bit position in real-time.

(2) The approximate sampling interval is $0.1-0.2$ seconds.

(3) The bolter is driven by air pressure rather than hydraulic pressure.

\subsubsection{Feedback Control System Developed by Structured Mining System, Inc., J. H. Fletcher \& Company and West Virginia University}

The feedback control system was initially developed by Structured Mining System, Inc. and J. H. Fletcher \& Co. in 1998. This system can be controlled automatically after inputting the pre-set drilling parameters. The major reason for developing this system is to improve drilling consistency and bit life ${ }^{[7]}$. In order to improve the performance of the data monitoring system and meet the requirements of the research project, Evaluation of Roof Bolting Requirements Based on In- mine Roof Bolter (sponsored by U.S.DOE under its IOF-Mining program ${ }^{[17]}$ ), West Virginia University has been cooperating with J. H. Fletcher \& Co. since 1999. Efforts have been made to improve the performance of the monitoring system, including the accuracy of measuring the bit position and torque.

The improved control is a result of the closed control loops for the feed and rotation. The sensors (such as thrust, torque, and position sensors) input information to the controller where the condition statements and subroutines act on the information to regulate the output to the control valve solenoids. The control system on the mast feed 


\section{Literature Review}

machines accepts information from a feed force cell located in the drill head trunnion, a rotation rate counter located in the drill head, and a vacuum transducer located on the valve mount tray.

The Feedback Control Station (Figure 3) has the following capabilities:
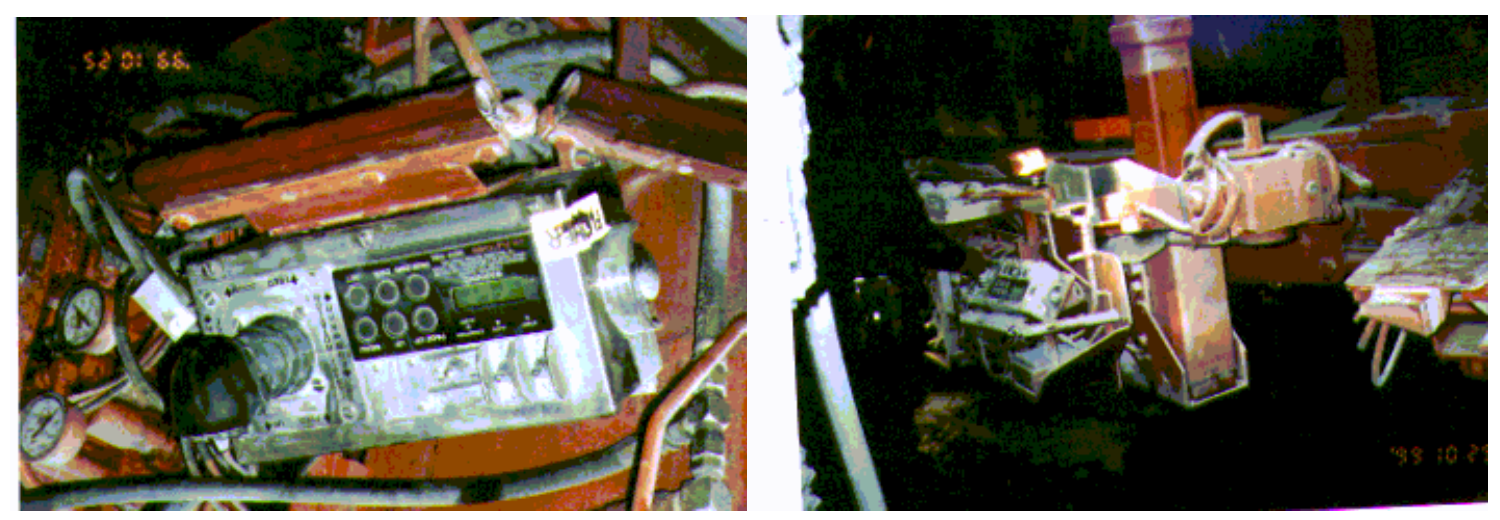

Figure 3 J.H. Fletcher's Bolter and Feedback Control Station ${ }^{[21]}$

(1) It can directly monitor the drilling parameters, such as feed force, penetration rate, rotation rate, drill bit position, and vacuum condition.

(2) All the parameters (i.e. thrust cap, penetration rate, and rotation rate) can be preset.

(3) The approximate sampling interval is 0.1 seconds.

Compared to the previous two realtime data acquisition systems developed by Parvus Corporation and Muroran Institute of Technology, the data acquisition system developed by J. H. Fletcher \& Co. and West Virginia University has its own features by considering the requirements of the project. There are three pre-setting modes, which are in conjunction with the pre-set thrust cap, can be chosen: Penetration rate is controlled; rotation rate is controlled; Penetration rate and rotation rate are controlled. 


\section{Literature Review}

\subsection{Analysis of Drilling Parameters in the Past}

In an attempt to find the relationship between drilling parameters and rock properties, there has been growing interest in the analysis of drilling parameters. Based on the techniques used in the past, the types of analysis can be divided into two groups: monovariant analysis and neural network. The mono-variant analysis used for the initial research of the drilling parameters is used to find the correlation between rock strength and a single specific drilling parameter, such as specific energy. Neural network analysis, a classification schema, such as self-organizing map or back-propagation, is used to classify or identify rock properties such as the rock type and locations of discontinuities.

Teale $^{[2]}$ conducted experiments to test and check the relationships between drilling parameters. It could be generalized as:

(1) The minimum specific energy could be reached when the specific energy approaches or is roughly equal to the compressive strength of the rock (Figure 4). The crushing strengths of the rock and concrete used are indicated in Figure 4. Except for the M3 bit in Pennant sandstone, there is a close correspondence between crushing strengths and the minimum specific energies attained.

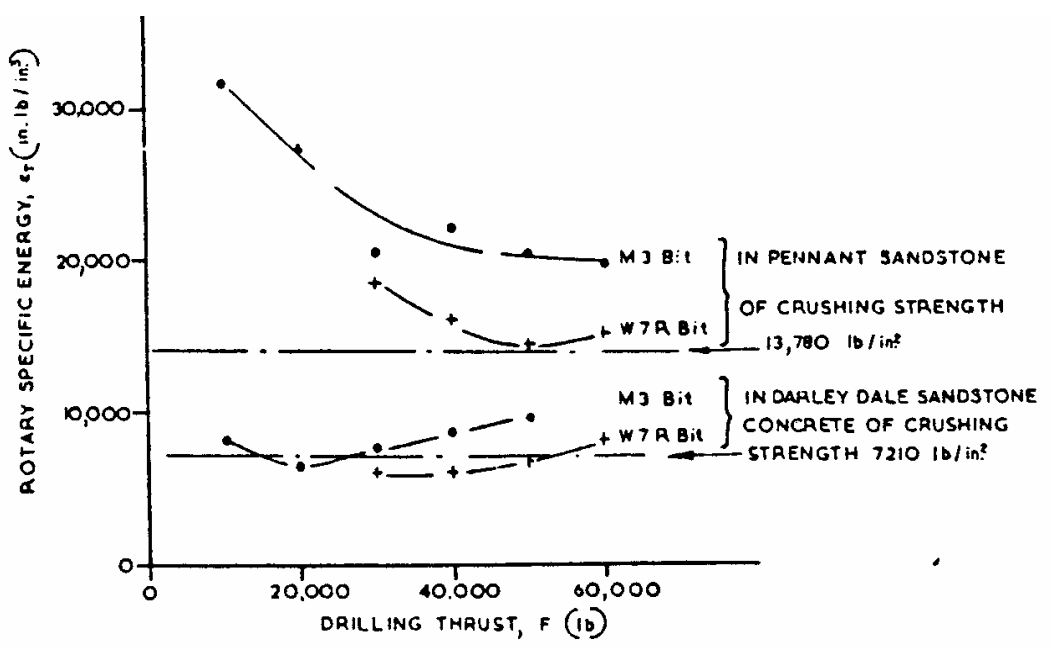

Figure 4 Specific energy for two roller bits in two media ${ }^{[2]}$ 


\section{Literature Review}

(2) While drilling one type of rock, the relationship between thrust and penetration rate is approximately linear at constant rotation rate.

(3) The minimum specific energy attained by a particular bit is determined by its geometry $^{[2]}$ (Figure 5). Figure 5 shows total specific energy plotted against penetration per revolution in Pennant sandstone. The percussive-rotary values shown represent three rotation speeds and two levels of percussive energy input. No significant differences can be detected for the different levels of these variables. This figure also shows a few points for rotary drilling in the same rock. Those representing pure rotary drilling with a percussive-rotary bit fall to a minimum which is on the same level as that obtained with this type of bit when percussion was used, whereas those representing rotary drilling with rotary bit are even lower ${ }^{[2]}$. This suggests that the minimum specific energy attained by a particular bit is determined by its geometry ${ }^{[2]}$.

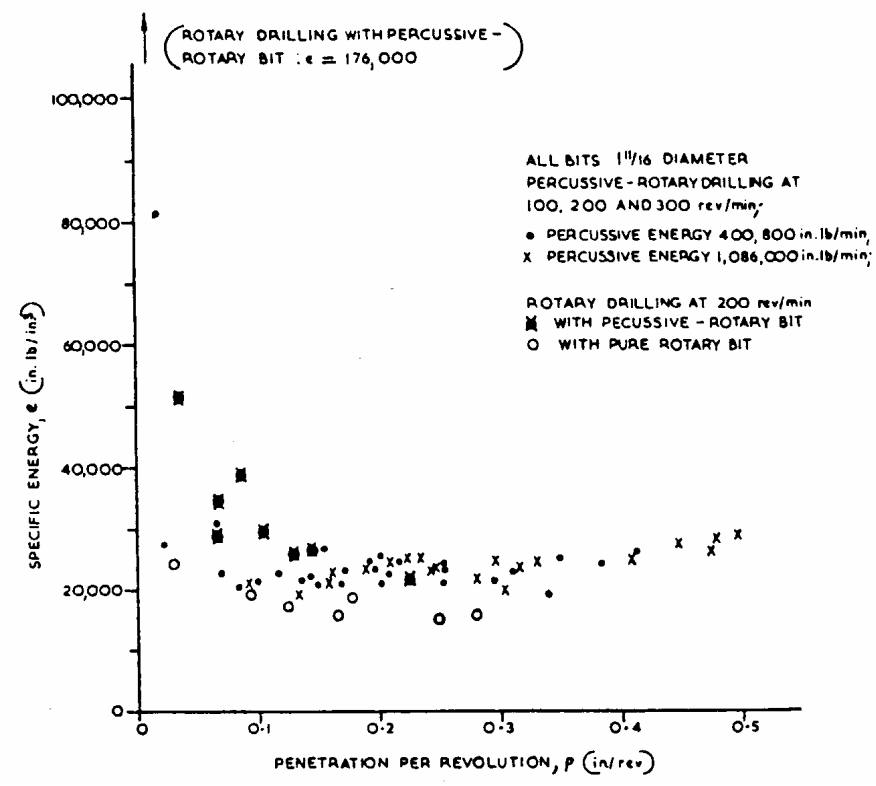

Figure 5 Specific energy and penetration per revolution for percussive-rotary and rotary drilling in Pennant sandstone ${ }^{[2]}$ 


\section{Literature Review}

(4) Rocks themselves vary so often that their strengths cannot be truly represented by a single number. Teale ${ }^{[2]}$ suggested that the ratio of specific energy to compressive strength might rise above 1.6 or fall below 0.8 .

The consideration made by Teale that specific energy was to be an intrinsic property such that the parameters of the breakage mechanism is negligible, is controversy. Rabia (1982) discussed specific energy criteria and stated that specific energy determined for rotary drills is considerably larger than that determined for percussive drills for the same $\operatorname{rock}^{[12]}$. Moreover, specific energy of rocks is sensitive to bit types. Specific energy determined in the laboratory and values determined in the field show percentage differences as much as $83 \%$. Therefore, it is considered that specific energy is not a fundamental intrinsic property of rock. However, in 1993, Jain, and Singh ${ }^{[12]}$ supported Teale's consideration that specific energy is an intrinsic property of rock and a reliable parameter for predicting rock drillability through their laboratory study by using drag-bit and micro-bit. The correlation results of laboratory drilling indicated that the specific energy varies exponentially with rock strength properties.

The expressions used for the calculated specific energy are shown in the following ${ }^{[12]}$ :

$$
\begin{aligned}
& S E_{v}(T)=\frac{2 \pi \cdot N \cdot T}{A \cdot P R} \\
& S E_{v}(W)=\frac{2.35 \cdot 60 \cdot W \cdot N}{D \cdot P R}
\end{aligned}
$$

where

$\mathrm{SE}_{\mathrm{v}}(\mathrm{T})$ - specific energy required to break the cementing bond of unit volume of rock material. It corresponds to the intrinsic property of the material.

$\mathrm{SE}_{\mathrm{v}}(\mathrm{W})$ - energy needed to compensate the thermal energy dissipated as a 


\section{Literature Review}

result of friction between bit surface and hole surface and vibration energy which denotes the total energy that depends on external factors like bit type, bit geometry, and flushing conditions.

$\mathrm{N}$ - bit rotational speed, rotation rate

$\mathrm{T}$ - torque, kg-meter

$\mathrm{PR}$ - penetration rate, $\mathrm{cm} / \mathrm{min}$ and

A - cross-sectional area, $\mathrm{cm}^{2}$

$\mathrm{W}$ - thrust, $\mathrm{kg}$

$\mathrm{D}$ - diameter of the bit, $\mathrm{mm}$

Based on the correlations between specific energy and compressive strength, shear

strength, tensile strength, and Schmidt hardness, Jain and Singh ${ }^{[12]}$ obtained the following multiple regression equations used to calculate the predicted specific energy

$S E_{v}(T)=69.958 \cdot \exp \left(0.0528 S c h .+0.0854 \sigma_{\tau}-0.0164 \sigma_{c}\right)$

Correlation coefficient $\mathrm{R}=0.9996$

$S E_{v}(W)=23.535 \cdot \exp \left(0.0082 S c h .+0.0051 \sigma_{\tau}-0.0066 \sigma_{t}\right)$

Correlation coefficient $\mathrm{R}=0.9968$

where

Sch. - Schmidt hardness number,

$\sigma_{\mathrm{c}}$ - Compressive strength, $\mathrm{kg} / \mathrm{cm}^{2}$,

$\sigma_{\tau}$ - Shear strength, $\mathrm{kg} / \mathrm{cm}^{2}$ and

$\sigma_{\mathrm{t}^{-}}$Tensile strength, $\mathrm{kg} / \mathrm{cm}^{2}$.

The calculated and predicted specific energy values are shown in Tables 2 and 3. 


\section{$\underline{2 \text { Literature Review }}$}

Table 2 Calculated specific energy $\mathrm{SE}_{\mathrm{V}}(\mathrm{T})$ and predicted specific energy $\mathrm{SE}_{\mathrm{v}}(\mathrm{T})$ from strength properties ${ }^{[12]}$

\begin{tabular}{|c|c|c|}
\hline Rock type & $\begin{array}{c}\text { Calculated specific energy } \\
\text { (MJ/cu.m) (Using equation 12) }\end{array}$ & $\begin{array}{c}\text { Predicted specific energy (MJ/cu.m) from } \\
\text { strength properties (Using equation 14) }\end{array}$ \\
\hline Makrana marble & 379.3 & 378.4 \\
\hline Chunar sandstone & 278.9 & 278.4 \\
\hline Damodar sandstone & 146.6 & 148.8 \\
\hline Kusunda sandstone & 117.8 & 116.6 \\
\hline Coal & 31.2 & 31.2 \\
\hline
\end{tabular}

Table 3 Calculated specific energy $\mathrm{SE}_{\mathrm{v}}(\mathrm{w})$ and predicted specific energy $\mathrm{SE}_{\mathrm{v}}(\mathrm{w})$ from strength properties ${ }^{[12]}$

\begin{tabular}{|l|c|c|}
\hline \multirow{2}{*}{ Rock type } & $\begin{array}{c}\text { Calculated specific energy } \\
\text { (MJ/cu.m) } \\
\text { (Using equation 13) }\end{array}$ & $\begin{array}{c}\text { Predicted specific energy (MJ/cu.m) from } \\
\text { strength properties } \\
\text { (Using equation 15) }\end{array}$ \\
\hline Makrana marble & 789.9 & 800.3 \\
\hline Chunar sandstone & 665.7 & 686.1 \\
\hline Damodar sandstone & 276.3 & 245.4 \\
\hline Kusunda sandstone & 148.5 & 162.5 \\
\hline Coal & 86.2 & 85.8 \\
\hline
\end{tabular}

The results obtained from Jain and Singh's experiments were excellent, but the reliability of equations 14 and 15 for predicting specific energy has never been validated in the field. Another problem with Jain and Singh's equation 13 in calculating the total energy required is that the unit of specific energy is $\mathrm{kg}$-revolution/ $\mathrm{m}^{2}$ rather than $\mathrm{kg} / \mathrm{m}^{2}$. In addition, rock strength, including compressive, tensile and shear strengths varies. By choosing a different number within the strength range, a different predicted specific energy could be reached. Although, Jain and Singh's work verified that specific energy varies exponentially with rock strength properties, it did not show how to determine compressive strength or shear strength 


\section{Literature Review}

Based on the analysis of specific energy, Reddish and Yasar ${ }^{[11]}$ tried to use a new strength index, gradient and/or stall penetration rate (See Figure 6), to correlate with rock strength while the operator could control thrust approaching a stall condition when thrust is excessive due to power limitations. The correlations are shown in Figures 7 and 8. It can be seen that with an increase in uniaxial compressive strength or Young's modulus, the corresponding specific energy at stall penetration rate increases. The regression coefficients for these correlations are very high.

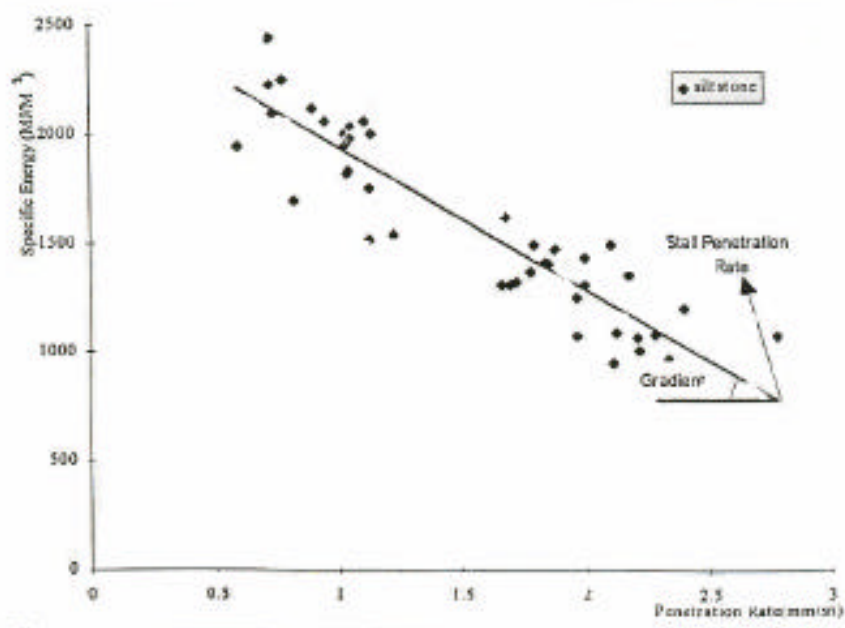

Figure 6 Gradient and stall penetration rate $\mathrm{e}^{[11]}$

Reddish and Yasar ${ }^{[11]}$ verified that specific energy could be one of the indices to identify rock properties. However, there are three things that make it difficult to apply the result in the field. First, the drill used for the laboratory test, was a modified industrial rotary hammer drill utilizing rechargeable $12 \mathrm{~V}$ Nicad batteries with a $10 \mathrm{~mm}$ masonry bit. However, the power and bit geometry used in the field are fairly different from the roof bolter with a drill bit at least $25 \mathrm{~mm}$ in diameter. This could result in a large difference while calculating specific energy, because a larger bit size can produce a smaller specific energy ${ }^{[12]}$. Secondly, it lacks the test drill speed or torque control. As a result, the 


\section{Literature Review}

attainment of peak penetration rate really depends on the operator who needs to apply judgment to determine the maximum thrust to avoid stall, but yet to ensure peak penetration. Thirdly, it is hard to operate in the field because of the judgment problem of the maximum thrust or peak penetration.

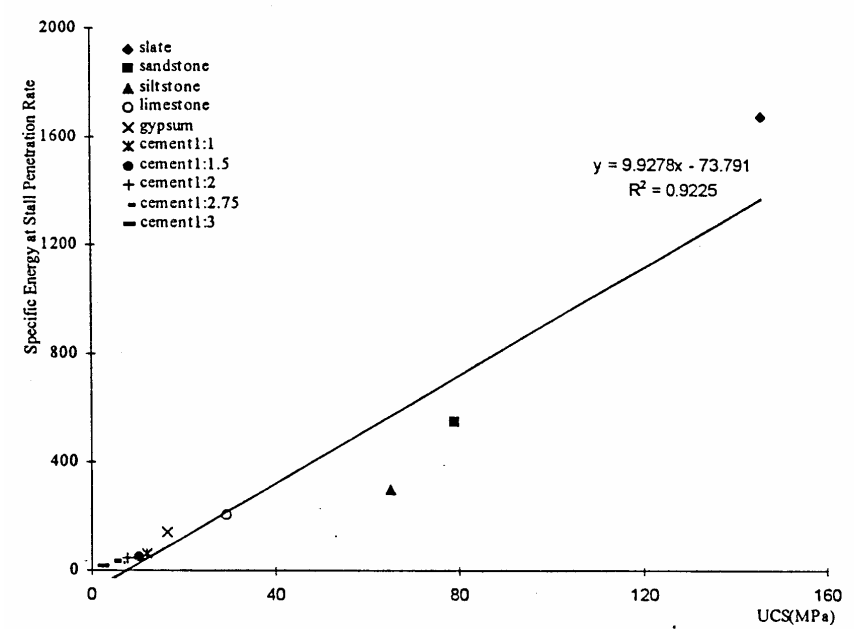

Figure 7 Specific energy at stall penetration rate and uniaxial compressive strength ${ }^{[11]}$

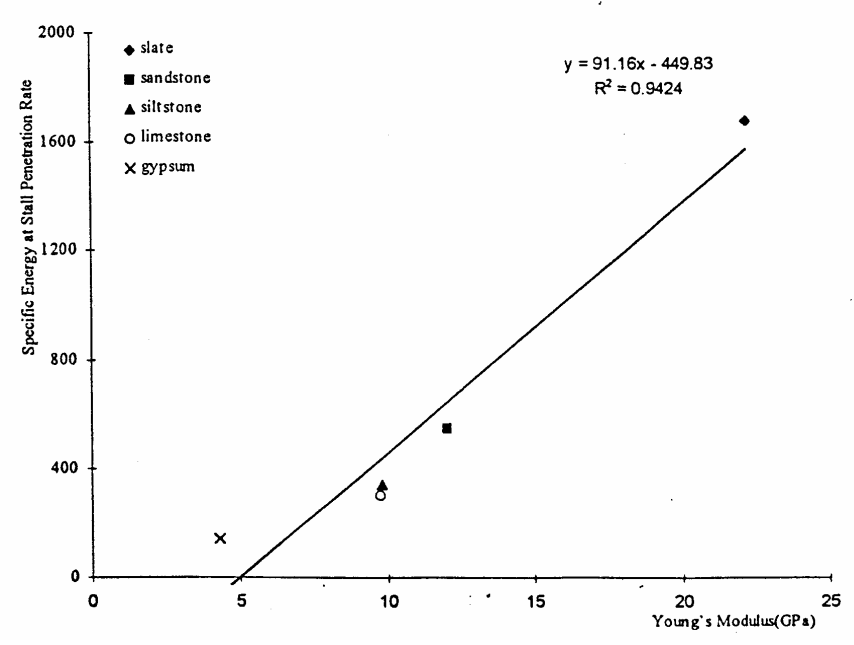

Figure 8 Specific energy at stall penetration rate and Young's modulus ${ }^{[11]}$

Briefly, all the above analyses tried to determine the relationship between rock properties and corresponding specific energy. However, most of the calculation in specific energy is achieved with the directly monitored mechanical drilling parameters 


\section{Literature Review}

that consist of a lot of uncontrolled noise components due to the adjustments of the hydraulic system and the discrepancies of the data log system. Therefore, the monovariant analyses that focus on calculating specific energy could not fully reflect rock properties, although specific energy could do it in theory. Artificial neural network, which involves multivariate analysis, has been widely used in the engineering field, such as pattern recognition, signal process, and so on In this research the simultaneous variations in thrust, torque, penetration and rotation rate make it possible in theory to do rock type classification and roof geology mapping. As a result, there has been growing interest in neural network or multivariate analysis of statistics. The following sections present some of the analysis methods used and some analysis results.

\subsubsection{The Analysis of Drilling Parameters Implemented by Spokane Research Center}

The Spokane Research Center developed an intelligent mine roof classifier (IMRC) that assists mining engineers in selecting roof bolts for ground control in coal mines. The schematic of the architecture of the IMRC is shown in Figure 9.

The capabilities of this expert system include: analysis of pull tests conducted on roof bolter, determination of anchorage capacity, evaluation of user input on support type and engineering characteristics, and development of geotechnical information of an entry cross-section based on input from the neural network and information from users.

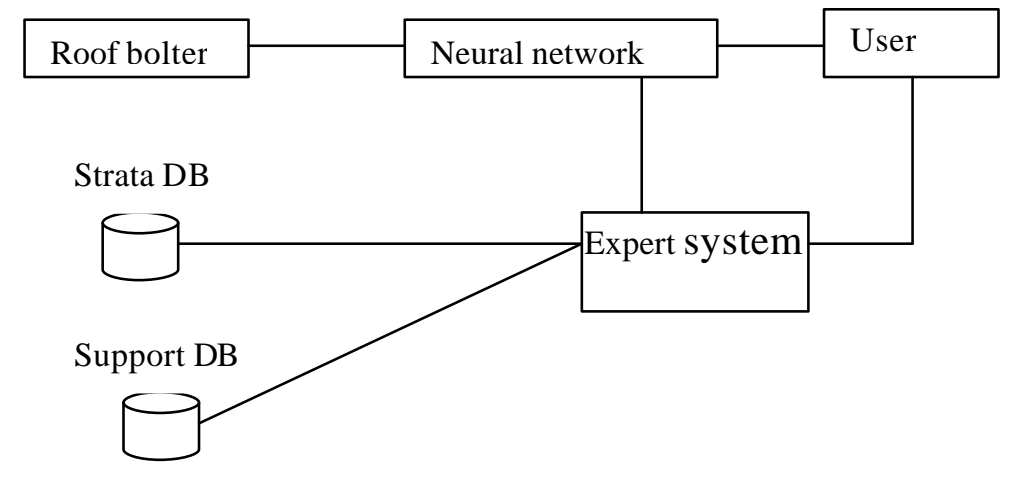

Figure 9 Schematic of IMRC ${ }^{[4]}$ 


\section{Literature Review}

The neural network used in this system is Self-organizing and Back-propagation, i.e., using the Self-organizing neural network to cluster drilling data vectors into reasonable patterns/classes, which can then be used in the back-propagation neural network in the development of supervised learning classification or prediction network,.

The self-organizing mapping (SOM) neural network, which is one type of unsupervised learnings, was used to train and classify drilling parameters by using the Euclidean distance to cluster ${ }^{[5]}$. By this neural network, 16 exemplar sets were obtained from 617 unclassified training patterns and specific energy was used to order the cluster numbers from the lowest value (0) to the highest (15) (see Table $4^{[5]}$ ).

Those 16 classes can be used in the development of the back-propagation network that is used in classification or prediction. The cluster numbers serve as relative strength index. In other words, the geological feature represented by cluster 3 was easier to drill through than the geological feature represented by cluster 5 . The higher the cluster number is, the harder to drill through the rock.

The back-propagation network consists of an input layer of torque, thrust, rotation rate, and penetration rate, two hidden layers, and an output layer that represents the mine roof feature space developed from the unsupervised neural work. Nonlinear learning was used to connect the input layer to the hidden layers. Linear learning was used to connect the second hidden layer to the output layer and the input vector to the output vector. The schematic of the typical neural network architecture is shown in Figure 10.

Table 4 Sixteen-exemplar sets obtained from 617 unclassified training patterns ${ }^{[5]}$

\begin{tabular}{|c|c|c|c|c|}
\hline Cluster No. & Penetration rate (mm/s) & Thrust (N) & $\begin{array}{c}\text { Drill speed } \\
\text { (rotation rate) }\end{array}$ & Torque (J) \\
\hline 0 & 2.6 & 1879 & 1.2 & 0.5 \\
\hline 1 & 2.8 & 622 & 47.3 & 7.1 \\
\hline 2 & 14.4 & 3776 & 82.8 & 20.8 \\
\hline 3 & 13.6 & 5088 & 171.3 & 27.9 \\
\hline
\end{tabular}




\section{Literature Review}

\begin{tabular}{|c|c|c|c|c|}
\hline 4 & 18.6 & 9230 & 434.2 & 46.3 \\
\hline 5 & 18.8 & 10,537 & 438.3 & 54.5 \\
\hline 6 & 15.3 & 4926 & 395.2 & 57.0 \\
\hline 7 & 9.3 & 2603 & 438.1 & 33.6 \\
\hline 8 & 19.6 & 11,652 & 434.8 & 69.6 \\
\hline 9 & 14.0 & 7759 & 394.8 & 63.3 \\
\hline 10 & 12.8 & 6559 & 407.4 & 56.6 \\
\hline 11 & 10.9 & 3765 & 447.6 & 45.4 \\
\hline 12 & 8.8 & 1443 & 409.5 & 43.8 \\
\hline 13 & 15.8 & 12,648 & 425.7 & 92.5 \\
\hline 14 & 15.2 & 14,018 & 436.3 & 91.8 \\
\hline 15 & 4.8 & 289 & 414.3 & 34.5 \\
\hline
\end{tabular}

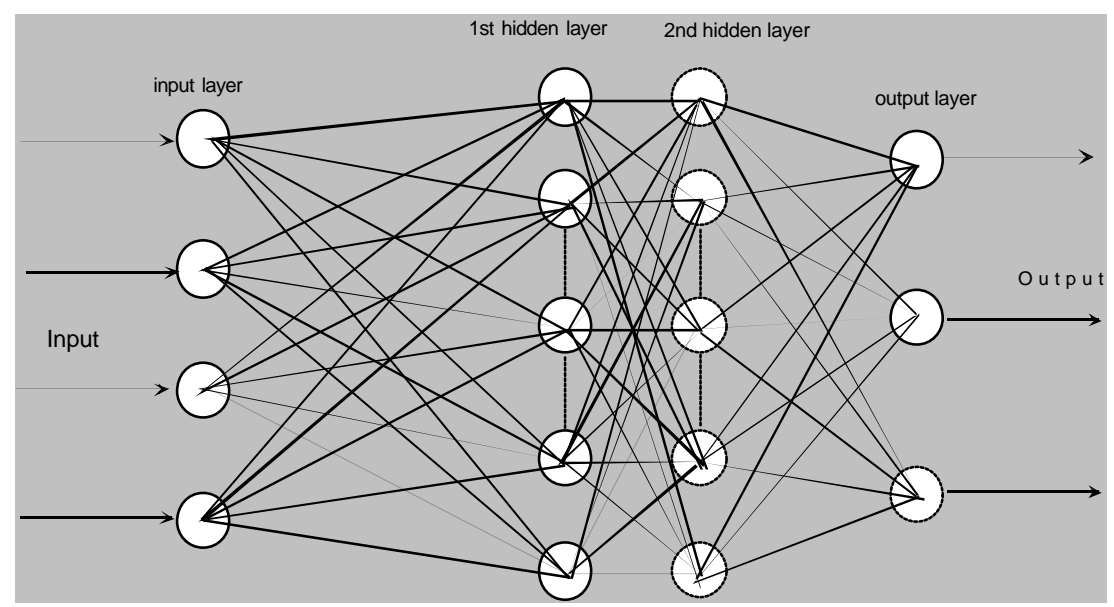

Figure 10 Typical back-propagation neuralnetwork architecture ${ }^{[4]}$

Figure 11 shows the output results from the back-propagation neural network and core logs ( $\mathrm{x}$ axis represents the specific energy of drilling (SED) whose unit is MPa and y axis represents the drilled hole length, about $0.95 \mathrm{~m}$ ). From this figure we can see that the output from the SOM neural network matched the core logs well except for scattered carbonaceous laminations that had two different strengths around the fracture. 


\section{Literature Review}

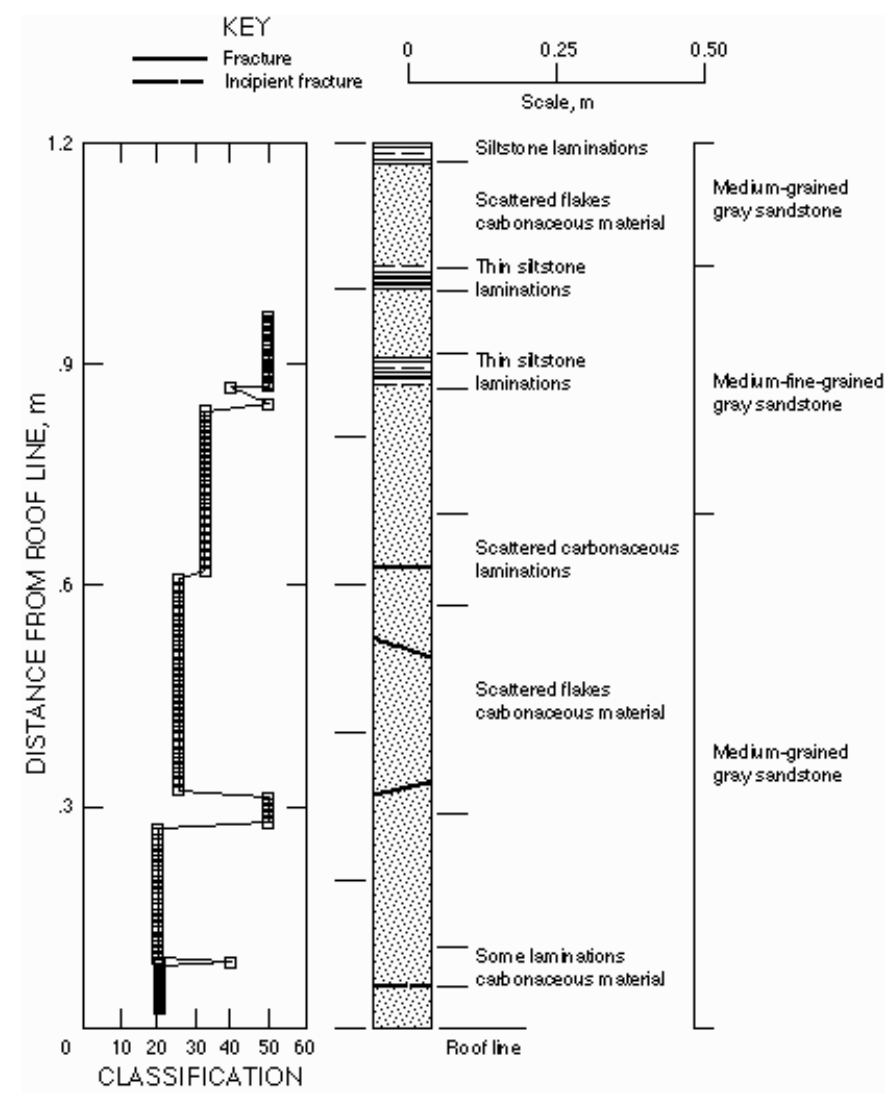

Figure 11 Comparison of neural network output for core hole ${ }^{[4]}$

This analysis system is a promising way to comprehensively analyze drilling parameters and predict rock properties. However, there are several problems with what the Spokane Research Center did:

(1) For self-organizing neural network, it is not best to use Euclidean distance to cluster, because the drilling parameters are dependent variables rather than independent variables. Instead, Mahalanobis distance, which considers the correlation among the data, should be employed.

(2) Although the cluster number served as a relative strength index, from neural network point of view, it does not supply the exemplars for discontinuities. Therefore, it is impossible to detect crack and layer interfaces using those 16 exemplars. 


\section{Literature Review}

(3) It does not supply a method by which the drillability of rock matches rock strength. Therefore it cannot be used to determine an effective parameter which could reflect rock properties for rock bolting design.

\subsubsection{Drilling Parameters Implemented by Itakura and his Colleagues $^{[14][15][16]}$}

Based on a series of laboratory tests, Itakua et al. ${ }^{[15]}$ developed 9 learning patterns for this neural network to classify and predict discontinuities. Meanwhile, rock types were classified using the average ratio of torque to thrust. The data analysis and geostructure mapping were divided into the following three steps ${ }^{[14]}[16]$.

The first step was the preparation of mechanical logging data. Time-series mechanical data are occasionally smoothed to eliminate noise from eccentric rotation by moving average and digital filter techniques. Next, the time-series data used only during rock cutting are extracted and transformed into log data based on the stroke changes. In this step, adaptive resonance theory $2^{[18]}$ (ART2) algorithm of neural network was applied for rough pattern classification. However, it was found that this algorithm was too flexible to extract the discontinuity pattern precisely from the mechanical data log. Therefore, ART2 was only used for the initial data from the site to roughly classify the patterns by setting the vigilance parameter of ART2 to a high value. After the classification by ART2, the patterns corresponding to the discontinuities in rock were selected for the learning data in the second step.

From the experimental results, torque and/or thrust was determined for use in classifying rock type by the average value of torque or thrust, or a combination of both. Six variation patterns, which show how torque or thrust varies with the variation of discontinuities, and of torque or thrust obtained from experimental data are summarized in Figure 12. If the variation pattern in Figure 13 is extracted from the mechanical data $\log$, the location of discontinuities can be found. 


\section{Literature Review}

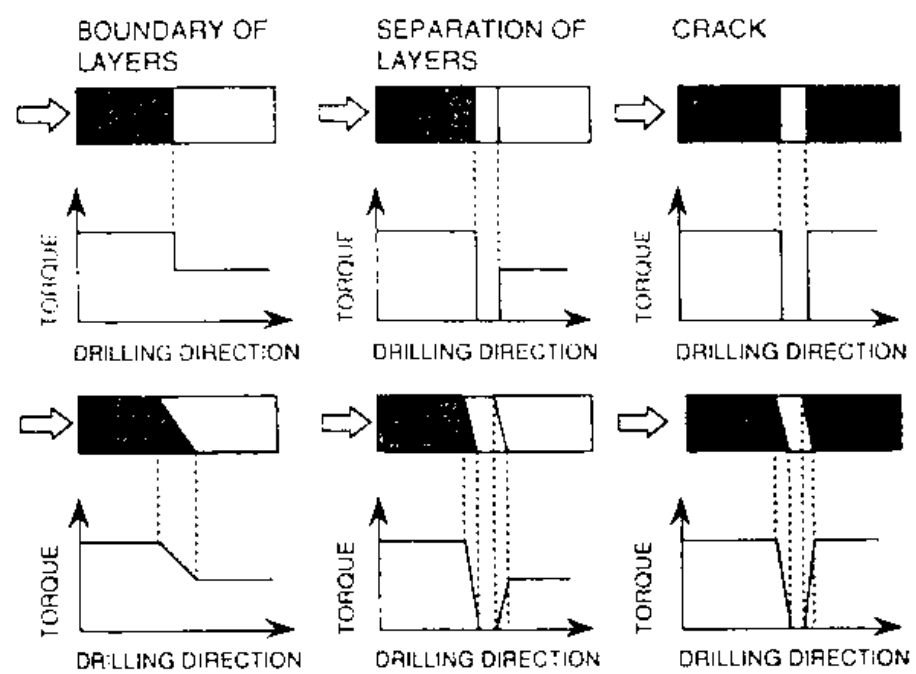

Figure 12 Theoretical patterns of mechanical data for various types of discontinuities ${ }^{[16]}$

The extracted variation patterns from a series of laboratory experiments were just typical variation patterns corresponding to discontinuities. For the field drilling, there would be more similar pattern. But, all the patterns shown in Figure 12 could be simplified into the nine training sets shown in Figure $13^{[18]}$ based on the variation trends of the drilling parameters while drilling in various discontinuities.

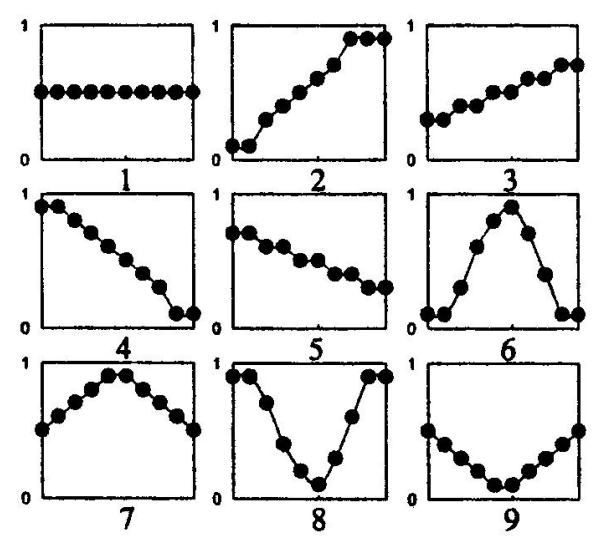

Figure 13 Simplified variation learning patterns of discontinuities for back-propagation neural network $^{[16]}$ 


\section{Literature Review}

In the second step, the neural network was used to extract the locations of discontinuities from the torque log. In this study, the simple back-propagation network (BP) was used. Data of the torque $\log$ at $15 \mathrm{~mm}$ intervals are categorized by the BP, and the location of the discontinuity is assumed to be at the middle point of each interval. In addition, nine simple patterns corresponding to the discontinuities were prepared for learning data of BP (see Figure 13). After detecting the locations of discontinuities, the discontinuities were classified into interfaces of rock layers, and fractures/cracks in rocks. This classification was carried out by comparing the average torque/thrust values before and after the discontinuity point and variation patterns of torque data were shown in Figure 12. If the difference between the average values of the ratio of torque to thrust before and after a discontinuity point was less than a certain threshold level, the point is judged to be the location of a crack in the rock. Furthermore, if a location that was judged to be an interface of layers that includes a pattern corresponding to a crack, it is regarded as a separation of layers (no examples shown in Itakua's papers).

Rock types were classified in terms of the difference in the average ratio of torque to thrust values, with consideration given to lithological data obtained from past core drilling. This classification is based on the fact that the harder the rock is, the higher the average ratio of torque to thrust becomes.

In the third step, a two- or three-dimensional geo-structure is recommended based on the average torque/thrust logs and the locations of discontinuities. Strata interface curves (planes in a 3-D geo-structure) are estimated by linear or spline interpolation by integrating all the data at the site, such as core drilling, non-core drilling and other exploration data. If the geo-structure is simple and the strata lie approximately horizontal, it is possible to automatically estimate the curves or planes by computer ${ }^{[15]}$. After the interface curves of the strata have been determined, the 2- or 3-D geo-structure is displayed using commercial software for image processing (Slicer 3D, FORTRAN Research LLC). 


\section{Literature Review}

Figure 14 shows typical torque logs during drilling at points along a line from the No. 1 hole to the No. 23 hole $^{[14]}$. The horizontal axis represents the distance along the entry and the vertical axis represents the hole depth. The unit of torque is represented by hydraulic pressure. Figure 15 shows torque/thrust $\operatorname{logs}$ of a core drilling ${ }^{[16]}$. Crack distribution of a core sample and a core sample image are also included in this figure. The dots represent the estimated discontinuities. Solid circles in the division of flat torque/thrust value imply cracks. Comparing the estimated estimated crack locations to core sample, some show good agreement. Furthermore, the ratio of torque to thrust can be used to reflect physical properties of rocks ${ }^{[16]}$.

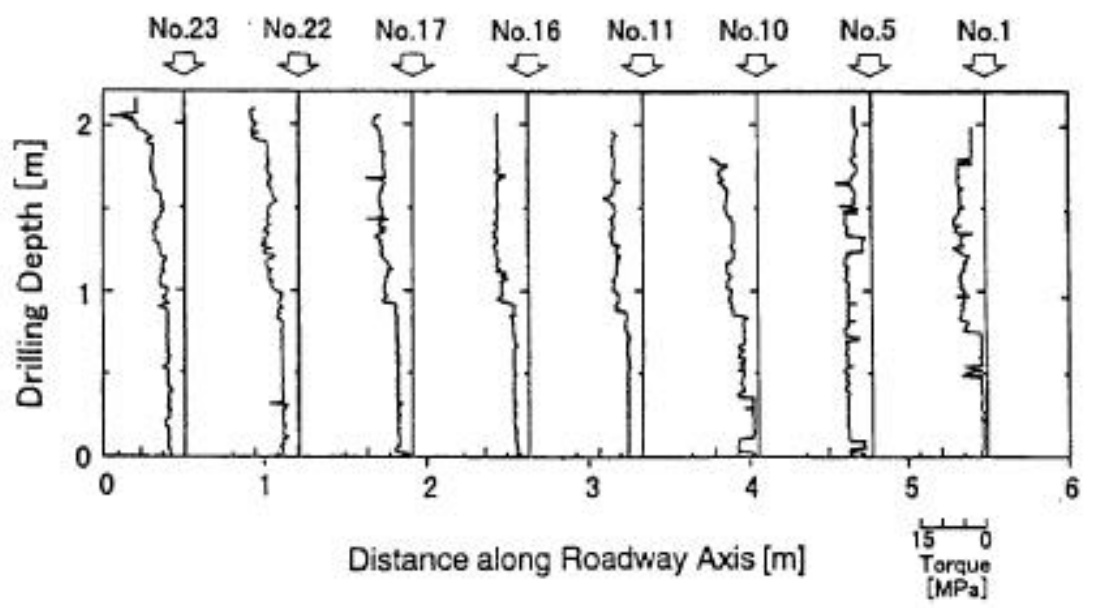

Figure 14 Typical torque logs and positions of drill holes ${ }^{[14]}$

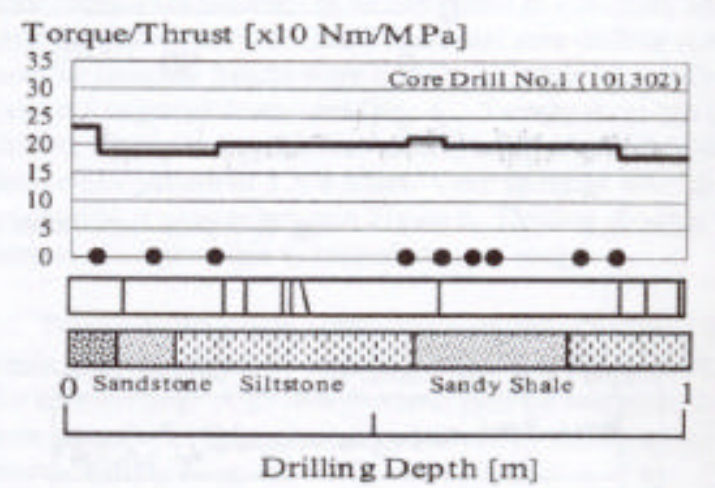

Figure 15 Torque/thrust logs of a core drilling ${ }^{[16]}$ 


\section{Literature Review}

In summary, in this study, the ratio of torque to thrust was considered as the major index to reflect geo-structure, including the distribution of discontinuities and rock types. The threshold or the difference of torque/thrust, however, used to determine rock type or discontinuities is hard to choose if there is no core drilling. However, the ideas of how to determine the locations of discontinuities and rock types are valuable and meaningful.

\subsubsection{Preliminary Analysis of Drilling Parameters Implemented by West Virginia University}

The major aim of the preliminary analysis was to determine how the data acquisition system would react while drilling from hard to soft rock and from soft to hard rock, and to verify the relationship between specific energy and the rock's compressive strength.

The drilling data used in this preliminary analysis was obtained from the experimental tests conducted in the spring of 2000 at J.H.Fletcher \& Company, Huntington, WV. Three blocks with embedded rock layers and one block with embedded horizontal fractures were drilled ${ }^{[10]}$. The manufactured roof rock blocks were designed and constructed by researchers at the Spokane Research Laboratory and are shown in Figures 16 and 17. Block 5 was designed to see how drilling parameters vary while drilling into simulated fractures constructed by embedding heavy weight illustration board (a dense cardboard). The simulated fractures covered the range of 1/8, 1/4, 3/8, 3/4, and 1 inch thick. The properties of rock and concrete of the drilled blocks are shown in Table 5.

Table 5 Average rock mechanics properties of rock units in the test blocks ${ }^{[10]}$

\begin{tabular}{|c|c|c|c|c|}
\hline & Unconfined & Brazilian tensile & Density, & Young's \\
& compressive strength & strength & $1 \mathrm{bs} / \mathrm{ft}^{3}$ & Modulus, \\
& $(\mathrm{psi})$ & $\left(10^{3} \mathrm{psi}\right)$ & & $\left(10^{6} \mathrm{psi}\right)$ \\
\hline Unit & 6,896 & 1.05 & 149 & 2.77 \\
\hline
\end{tabular}




\section{Literature Review}

\begin{tabular}{|c|c|c|c|c|}
\hline Light brown sandstone & 27,359 & 1.93 & 158 & 2.34 \\
\hline Brown sandstone & 9,995 & 0.93 & 160 & 1.94 \\
\hline White marble & 17,418 & 1.37 & 171 & 2.48 \\
\hline Argillite & 20,445 & 1.04 & 182 & 4.24 \\
\hline Hi-strength concrete & 2,830 & N/A & 159 & 20.7 \\
\hline Embedded concrete & 4,020 & 0.47 & 132 & 0.65 \\
\hline Embedded concrete in \\
blocks with fractures
\end{tabular}

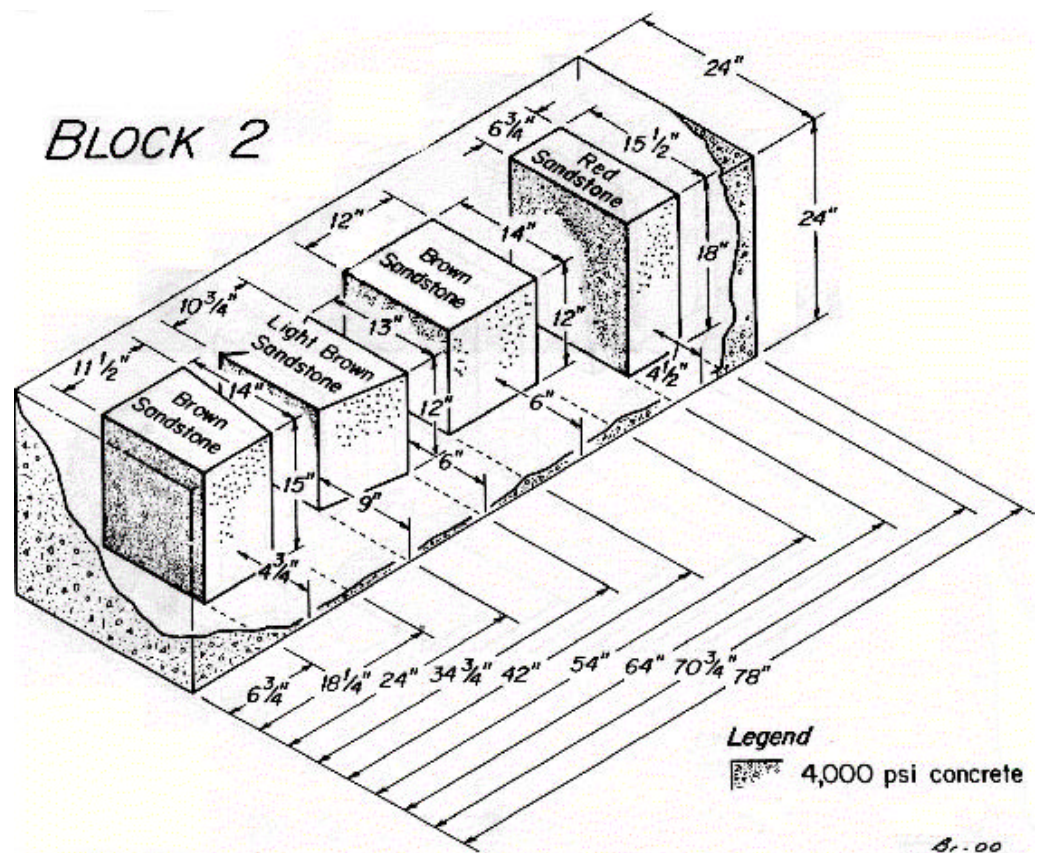




\section{Literature Review}

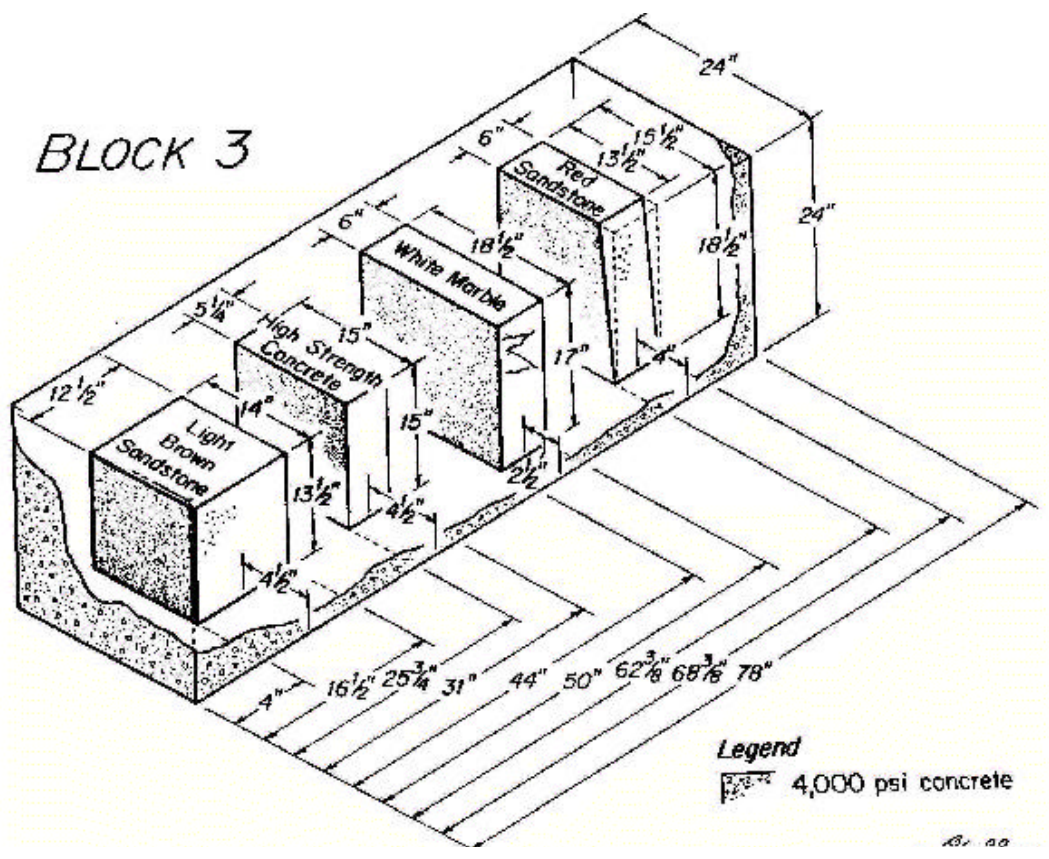

Figure 16 Schematics of the manufactured blocks 2 and 3

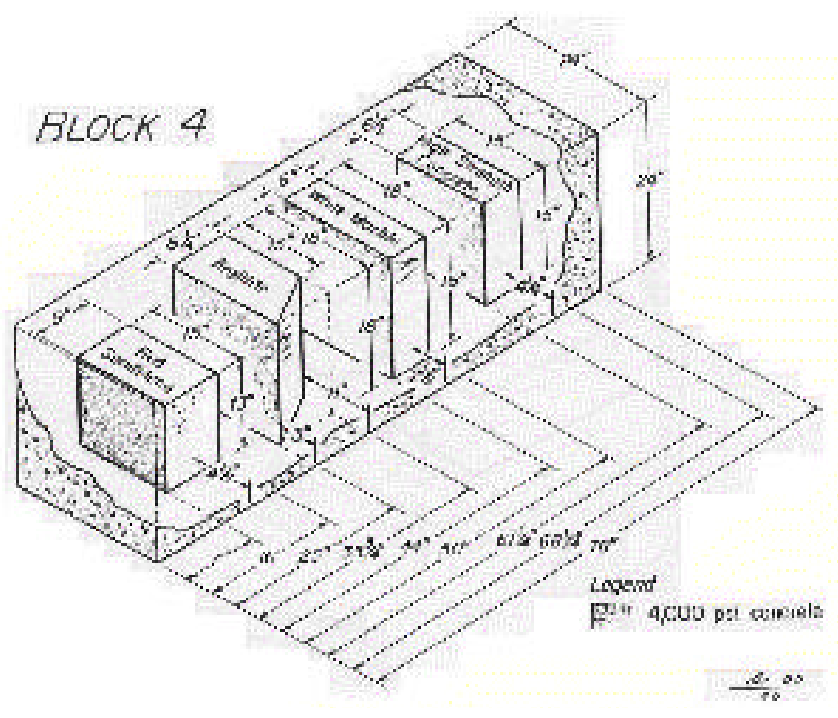




\section{Literature Review}

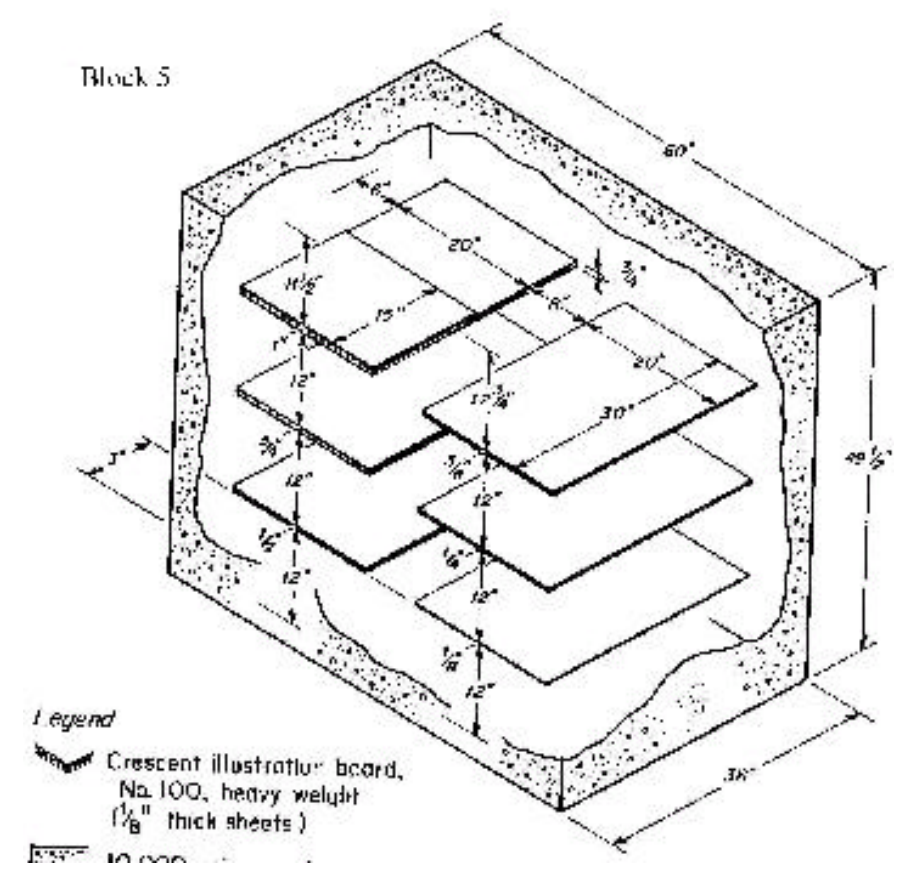

Figure 17 Schematics of the manufactured rock layer block 4 and block 5 with fractures

\subsubsection{Variations in Drilling Parameters While Drilling}

It was important to know how drilling parameters vary while drilling in different rocks. Experimental results showed that drilling parameters would change while drilling in different rocks. Consequently, drilling parameters could be used to reflect rock properties, such as the locations of interfaces, discontinuities, and the rock strength.

Figure 18 shows how the drilling parameters: thrust, penetration rate, torque, rotation rate, and specific energy (SED) vary while drilling rock layers. This figure was obtained from one of the drill holes from block 2 , and the penetration rate for this drilling hole was 


\section{Literature Review}

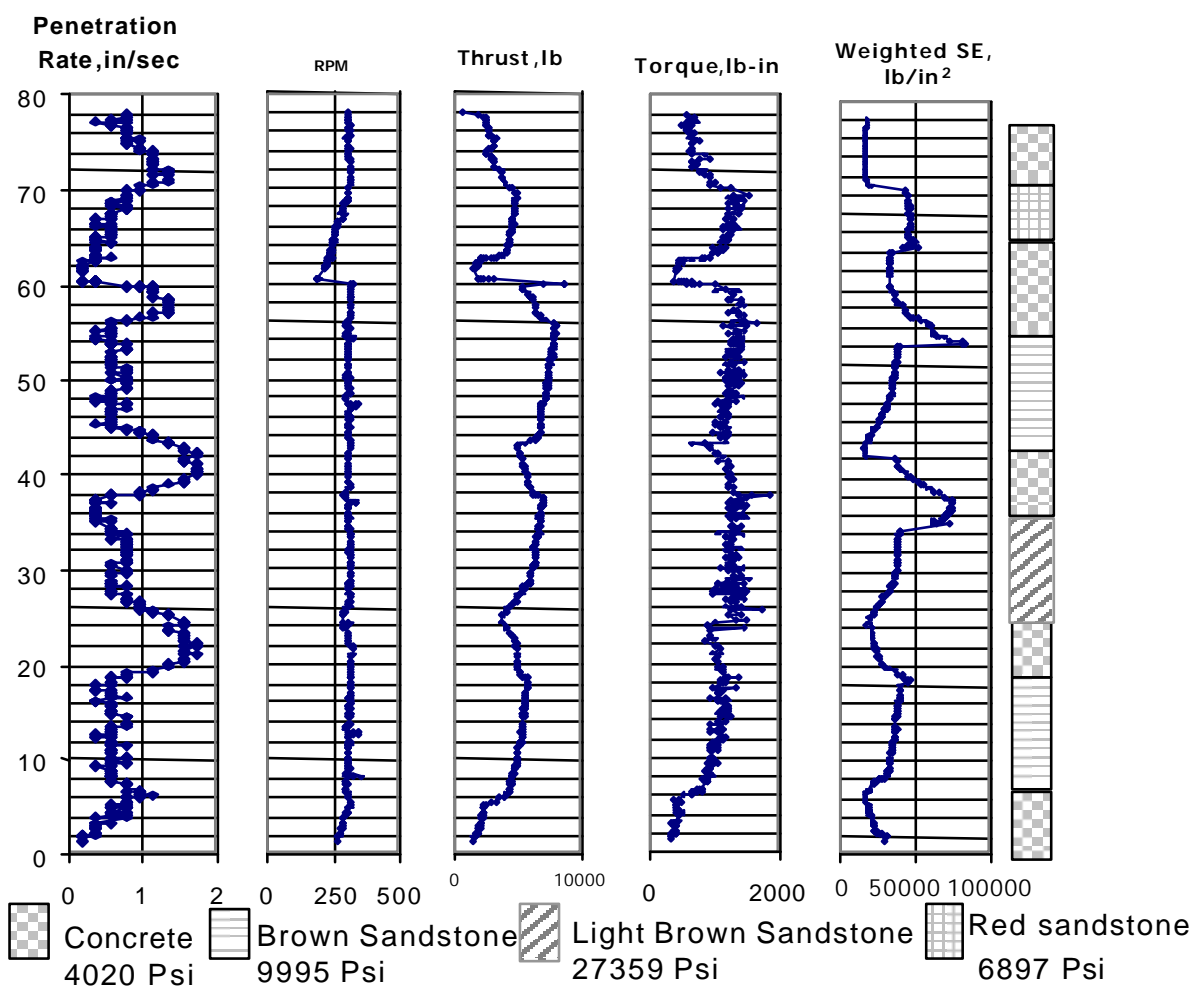

Figure 18 An example of the drilling parameters for manufactured block with rock layers ${ }^{[10]}$

set at $0.6 \mathrm{in} / \mathrm{sec}$ and the rotation rate was set at 300 . The variation of the penetration rate was similar to what was measured during all the laboratory experiments as it fluctuates considerably due to the varied rock properties ${ }^{[10]}$, although it was pre-set as constant. Comparatively, rotation rate remains fairly constant except for small fluctuations while drilling near the edge of rock layers. All five drilling parameters shown in Figure 18 varied, either increasing or decreasing while drilling from hard to soft rock or drilling from soft to hard rock. After extracting the variation patterns by studying the collected data for each hole (blocks 2, 3, and 4, a total of 15 holes), the frequency for each variation pattern was counted. The results are shown in Table 6. Eight different patterns were noted while drilling from hard to soft rock. Pattern number 8 was the most dominant variation trend 


\section{Literature Review}

Table 6 Variation trends of drilling parameters while drilling in manufactured blocks with embedded rock layers

\begin{tabular}{|c|c|c|c|c|c|c|c|c|}
\hline $\begin{array}{l}\text { Direc- } \\
\text { tion } \\
\end{array}$ & Pattern & Thrust & Torque & $\begin{array}{c}\text { Penetration } \\
\text { Rate } \\
\end{array}$ & $\begin{array}{c}\text { rotation } \\
\text { rate }\end{array}$ & SED & $\begin{array}{c}\text { Fre- } \\
\text { Quency }\end{array}$ & $\begin{array}{l}\text { Percen- } \\
\text { tage (\%) }\end{array}$ \\
\hline \multirow{3}{*}{ From } & 1 & $1^{\text {st } \downarrow \text { then } \uparrow}$ & $1^{\text {st }} \downarrow$ then $\uparrow$ & $\uparrow \uparrow$ & $\mathrm{NC}$ & $1^{\text {st }} \downarrow$ then $\uparrow$ & $\overline{1}$ & 2.38 \\
\hline & 2 & $\uparrow$ & $\downarrow$ & $1^{\text {st } \uparrow}$ then $\downarrow$ & $\mathrm{NC}$ & $\mathrm{NC}$ & 2 & 4.76 \\
\hline & 3 & $\downarrow$ & $\downarrow$ & $\downarrow$ & $\mathrm{NC}$ & $\downarrow$ & 2 & 4.76 \\
\hline \multirow{2}{*}{ Hard } & 4 & 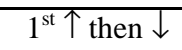 & $1^{\text {st }} \uparrow$ then $\downarrow$ & $\mathrm{NC}$ & $\mathrm{NC}$ & 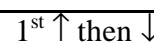 & 3 & 7.14 \\
\hline & 5 & $\uparrow$ & $\uparrow$ & $1^{\text {st }} \uparrow$ then $\downarrow$ & $\mathrm{NC}$ & $\downarrow$ & 3 & 7.14 \\
\hline to Soft & 6 & $\uparrow$ & $\uparrow$ & $\uparrow$ & $\mathrm{NC}$ & $\uparrow$ & 4 & 9.52 \\
\hline \multirow{2}{*}{ Rock } & 7 & $\uparrow$ & $\uparrow$ & $\uparrow$ & $\mathrm{NC}$ & $\downarrow$ & 4 & 9.52 \\
\hline & 8 & $\downarrow$ & $\downarrow$ & $1^{\text {st } \uparrow \text { then } \downarrow}$ & $\mathrm{NC}$ & $\downarrow$ & 23 & 54.76 \\
\hline From & 1 & $\uparrow$ & $\mathrm{NC}$ & $1^{\text {st }} \downarrow$ then $\mathrm{NC}$ & $\mathrm{NC}$ & $\uparrow$ & 1 & 3.23 \\
\hline \multirow{2}{*}{ Soft to } & 2 & $\uparrow$ & $\uparrow$ & $\mathrm{NC}$ & $\uparrow$ & $\uparrow$ & 1 & 3.23 \\
\hline & 3 & $\uparrow$ & $*$ & $*$ & $\mathrm{NC}$ & $*$ & 1 & 3.23 \\
\hline \multirow[t]{2}{*}{ Hard } & 4 & $\uparrow$ & $\downarrow$ & $\downarrow$ & $\mathrm{NC}$ & $*$ & 1 & 3.23 \\
\hline & 5 & $\uparrow$ & $\uparrow$ & $\downarrow$ & $\downarrow$ & * & 1 & 3.23 \\
\hline \multirow[t]{4}{*}{ Rock } & 6 & $\uparrow$ & $\uparrow$ & $\uparrow$ & $\uparrow$ & $\mathrm{NC}$ & 2 & 6.45 \\
\hline & 7 & $1^{\mathrm{st}} \uparrow$ then $\mathrm{NC}$ & $\uparrow$ & 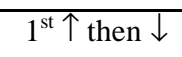 & $\mathrm{NC}$ & $1^{\text {st }} \downarrow$ then $\uparrow$ & 3 & 9.68 \\
\hline & 8 & $\uparrow$ & $\uparrow$ & $\mathrm{NC}$ & $\mathrm{NC}$ & $\uparrow$ & 4 & 12.90 \\
\hline & 9 & $\uparrow$ & $\uparrow$ & $\downarrow$ & $\mathrm{NC}$ & $\uparrow$ & 17 & 54.84 \\
\hline
\end{tabular}

Legend: $\uparrow=$ increase, $\downarrow=$ decrease, $\mathrm{NC}=$ no change, ${ }^{*}=$ no dominant trend

with 23 occurrences, representing $54.76 \%$ of the cases. This pattern showed that there was a decrease in thrust, torque and specific energy of drilling (SED), an increase then decrease in penetration rate and (almost) no change in the rotation rate. Of the remaining 7 patterns, none had a percentage of more than $10 \%$. For those variations from soft to hard rocks, 9 patterns were identified. The dominant pattern, accounting for $54.84 \%$ of the occurrences, is characterized by increase in thrust, torque and SED, a decrease in the penetration rate and no change in the rotation rate. Comparing pattern number 8 (drilling form hard to soft rock) with pattern 9 (drilling from soft to hard rock) shows that the variation trends reversed.

Another important feature of the transition phase of the drilling parameters either from hard to soft rock or from soft to hard rock was that the starting point was not right at the 


\section{Literature Review}

interface of the rock layers but near the edges. In other words, the drilling parameters start to vary at a certain distance from the interfaces of rock layers (Figure 18).

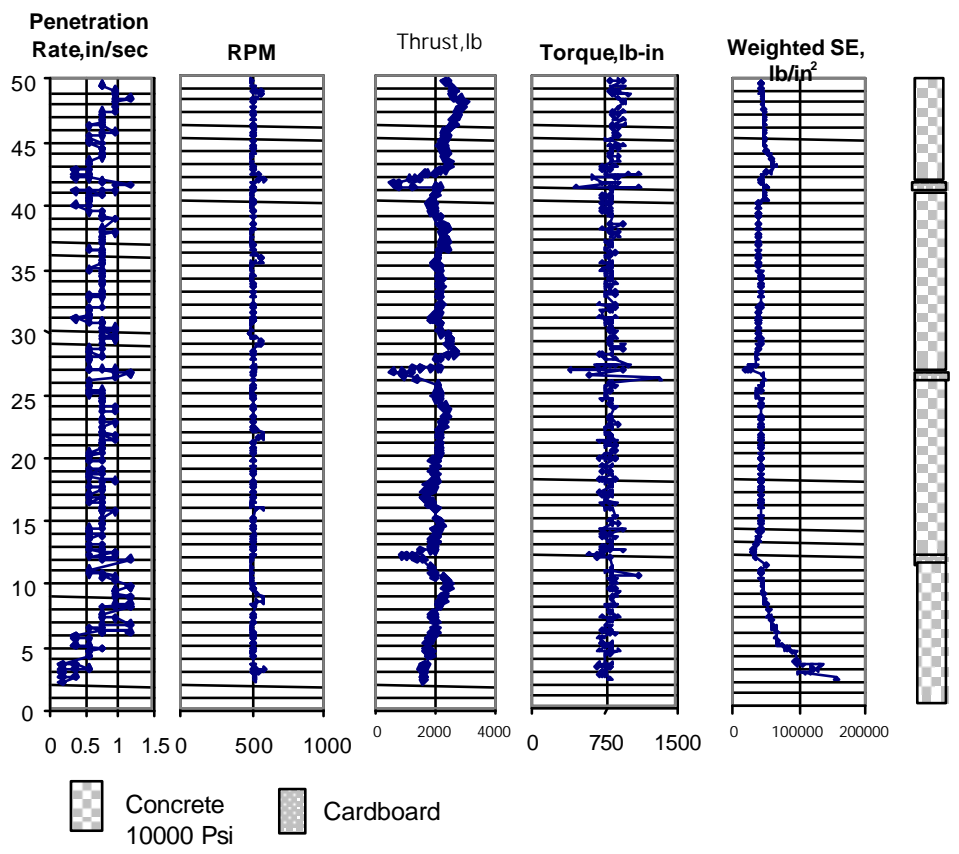

Figure 19 An example of the drilling parameters for manufactured blocks with simulated

$$
\text { fractures }{ }^{[10]}
$$

Figure 19 shows how the drilling parameters: thrust, penetration rate, torque, rotation rate, and specific energy of drilling (SED) vary while drilling the manufactured block with simulated fractures. This data was obtained from one of the drilling holes in block 5 . The penetration rate for drilling this hole was set at $0.6 \mathrm{in} / \mathrm{sec}$ and the rotational speed was set at 500 rotation rate. While the penetration rate fluctuated during the drilling process, it was more consistent than what was observed while drilling in the blocks with the rock layers. The transition zones around the edges of concrete layers were smaller than that of rock layers since the strength of the concrete layers between the cardboard layers was same.

Using the same process as with the rock layer blocks, the variation patterns in transition zones were identified and counted (Table 7). For those cases in which drilling 


\section{Literature Review}

transitioned from rock into a fracture, six patterns were identified. Pattern number 6 was the dominant pattern, accounting for about $65 \%$ of the cases. Pattern number 6 is characterized by decreases in thrust, torque and SED, an increase in the penetration rate and no change in the rotation rate, similar to pattern number 8 while drilling from hard to soft rock. For drilling transitions from fractures back into concrete, eight patterns were identified. Pattern number 8 was the dominant pattern, accounting for $61.77 \%$ of the occurrences. It was characterized by increase in thrust, torque and SED, a decrease in the penetration rate and no change in the rotation rate, similar to pattern number 9 while drilling from soft to hard rock.

The previous analyses show that, while drilling from hard to soft rock, the dominant variation pattern, which accounted for more than half of the occurrences, is a decrease in thrust, torque and specific energy, and an increase in the penetration rate. As would be reasonably expected, while drilling from soft to hard rock, the dominant variation pattern, which was also accounted for more than half of the occurrences, is an increase in thrust, torque and specific energy, and a decrease in the penetration rate. These results show that the feedback control system could effectively adjust itself in terms of change of rock types or geological conditions and that the drilling parameters will vary as observed above. In addition, it can be observed from the above drilling analysis that rock properties have critical influence on drilling parameters such as thrust, torque, penetration rate and rotation rate. These variations in drilling parameters makes possible the use of drilling parameters to map rock properties while drilling.

Table 7 Variation trends of drilling parameters while drilling in manufactured blocks with simulated fractures

\begin{tabular}{|c|c|c|c|c|c|c|c|c|}
\hline $\begin{array}{c}\text { Direc- } \\
\text { tion }\end{array}$ & Pattern & Thrust & Torque & Rate & rate & SED & Frequency & $(\%)$ \\
\hline \multirow{3}{*}{ From } & 1 & $\downarrow$ & $\downarrow$ & $*$ & $*$ & $*$ & 1 & 2.94 \\
\cline { 2 - 9 } Rock to & 2 & $\mathrm{NC}$ & $\mathrm{NC}$ & $*$ & $*$ & $*$ & 1 & 2.94 \\
\cline { 2 - 9 } Crack & 3 & $\downarrow$ & $1^{\text {st } \uparrow \text { then } \downarrow}$ & $\uparrow$ & $\mathrm{NC}$ & $\downarrow$ & 1 & 2.94 \\
\cline { 2 - 9 } & 4 & $\downarrow$ & $\mathrm{NC}$ & $\uparrow$ & $\mathrm{NC}$ & $\downarrow$ & 4 & 11.77 \\
\cline { 2 - 10 } & 5 & $*$ & $*$ & $*$ & $*$ & $*$ & 5 & 14.71 \\
\hline
\end{tabular}




\section{Literature Review}

\begin{tabular}{|c|c|c|c|c|c|c|c|c|}
\hline & 6 & $\downarrow$ & $\downarrow$ & $\uparrow$ & $\mathrm{NC}$ & $\downarrow$ & 22 & 64.71 \\
\hline \multirow{5}{*}{ From } & 1 & $\uparrow$ & $\uparrow$ & $*$ & $*$ & $*$ & 1 & 2.94 \\
\cline { 2 - 10 } to Rock & 2 & $\uparrow$ & $\downarrow$ & $\downarrow$ & $\mathrm{NC}$ & $*$ & 1 & 2.94 \\
\cline { 2 - 10 } & 3 & $\downarrow$ & $\uparrow$ & $\uparrow$ & & $\uparrow$ & 1 & 2.94 \\
\cline { 2 - 10 } & 4 & $\uparrow$ & $\uparrow$ & $\uparrow$ & & $\uparrow$ & 1 & 2.94 \\
\cline { 2 - 10 } & 5 & $\uparrow$ & $\mathrm{NC}$ & $*$ & $\mathrm{NC}$ & $\uparrow$ & 2 & 5.88 \\
\cline { 2 - 10 } & 6 & $\mathrm{NC}$ & $\mathrm{NC}$ & $*$ & $*$ & $*$ & 2 & 5.88 \\
\cline { 2 - 10 } & 7 & $*$ & $*$ & $*$ & $*$ & $*$ & 5 & 14.71 \\
\hline
\end{tabular}

Legend: $\uparrow=$ increase, $\downarrow=$ decrease, $\mathrm{NC}=$ no change, ${ }^{*}=$ no dominant trend

\subsubsection{Relationship between Specific Energy and Rock Strength}

The specific energy of drilling (SED) as defined by Teale ${ }^{[2]}$ was correlated with rock strength from the drilled blocks 2, 3 and 4. Table 8 shows the range of specific energy for different rock types. The range for each rock type was determined from the 15 holes drilled in blocks 2,3 , and $4^{[10]}$.

Table 8 Range of specific energy for rock layers in blocks 2 - 4

\begin{tabular}{|c|c|c|c|c|c|c|}
\hline Rock & Concrete & Red sandstone & Brown & White marble & Argillite & Light brown \\
type & & & sandstone & & & sandstone \\
\hline SED & $15943 \sim 84762$ & $15265 \sim 57428$ & $20197 \sim 87815$ & $20983 \sim 86104$ & $13489 \sim 87968$ & $15934 \sim 92964$ \\
\hline
\end{tabular}

Table 8 shows that the ranges of SED for different rock types overlap each other. The reason why overlap occurs is due to the anisotropic and the varied particle size, and the drilling inefficiencies ${ }^{[10]}$. In addition, the specific energy of drilling, or the calculated specific energy, using the monitored mechanical parameters, was not completely applied to drilling the rock. Part of it was consumed in the drill to rock and in moving the drill head and rod up in the form of kinetic and potential energies. These extra consumptions of energy made the calculated value of SED varied considerably, even when drilling within one type of rock. These factors cause all the drilling parameters, such as specific energy, to vary up and down even in one type of rock. 


\section{Literature Review}

The correlated relationship between the average SED and the average uniaxial compressive strength (UCS) of rocks is shown in Figure 20. In order to get the average SED of each rock type, the corresponding data sets for a certain rock type in each hole were extracted and grouped them together. A linear relationship was obtained with a correlation coefficient of 0.7393 .

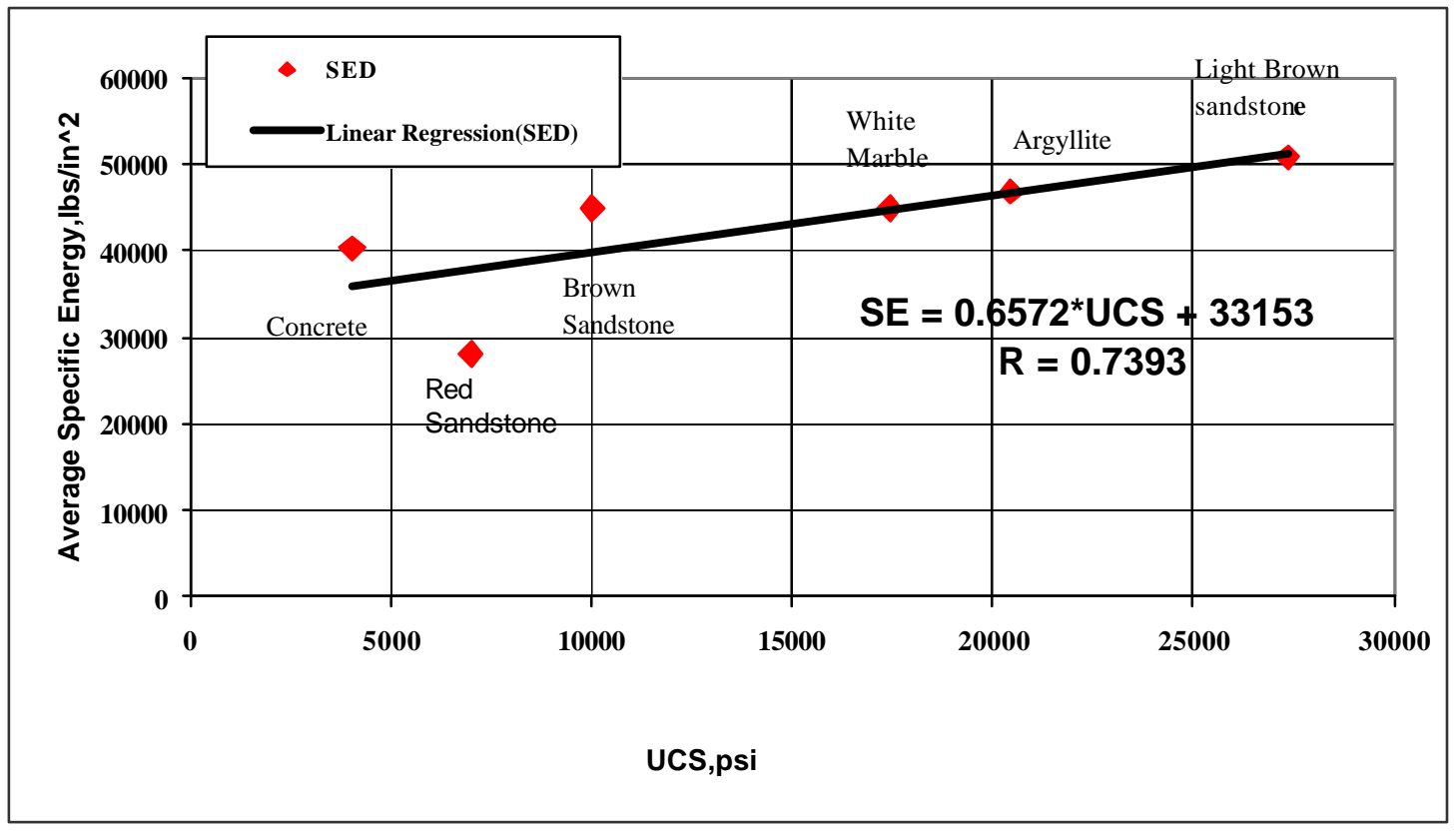

Figure 20 Correlation between SED and UCS of rock layers in blocks 2, 3, and 4

\subsection{Summary of the Literature Review}

It can be concluded from the literature review that

(1) The relationship between specific energy of drilling and rock properties was established and specific energy can be used as one of the rock indices to reflect rock properties. However, the specific energy of drilling, or the calculated specific energy, using the monitored mechanical parameters, was not completely applied to drilling the rock. Part of it was consumed in the drill to rock and in 


\section{Literature Review}

moving the drill head and rod up in the form of kinetic and potential energies. These extra consumptions of energy made the calculated value of SED varied considerably, even when drilling within one type of rock.

(2) The research method, developed by Spoken Research Center, combining a selforganizing map and back-propagation neural network, is very promising and instructional. However, it could not provide detailed rock properties, such as the locations of discontinuities and the rock strength, except for a relatively vague measure of drillability.

(3) The method developed by Itakura and his colleagues ${ }^{[14][15][16]}$ simplified the variation trends of torque into 9 patterns (Figure 13) to prepare training data for the back-propagation neural network to detect discontinuities, which is very meaningful and valuable. However, it is still difficult to apply to a roof bolter for the following reasons: (1) The hydraulic systems in the Wombat L.P. roof bolter (Figure 2) and Fletcher's roof bolter (Figure 3) are different, including the way in which thrust and torque are controlled, (2) The variation learning patterns of discontinuities are influenced by the hydraulic system of roof bolters which vary from roof bolter to roof bolter and manufacture to manufacture, and (3) The problem of how to determine the threshold of thrust/torque has not been solved with confidence.

(4) None of the past analysis considered how to eliminate, alleviate or avoid the effects of the energy lost and the adjustment of hydraulic system, even though these are very important parameters for detecting the locations of discontinuities and for determining the training patterns used for neural network analysis. 


\section{Research Objectives and Scope and Methodology}

\subsection{Objectives}

The major objective of this research is to map the geo-mechanical properties of an entry roof for roof bolting design. These rock properties include:

(1) Locations of interfaces between rock layers and discontinuities within one rock layer;

(2) Possible rock type.

\subsection{Scope of Research}

This research will concern the following areas:

(1) Determine and/or develop the most suitable drilling parameter(s) and develop algorithms to detect the locations of discontinuities.

(2) Analyzing the energy lost during the drilling process, such as drill-to-rock energy loss, work done on the drill head and rod in the forms of kinetic and potential energies, and thermal energy caused by the friction between the drill bit and the rock.

(3) Classify rock types by using supervised discriminant analysis.

\subsection{Methodology Used in This Research}

In order to develop an effective method to identify rock properties, laboratory and underground testing, data filtering and smoothing, and supervised discriminant analysis will be employed. 


\section{Research Objectives and Scope and Methodology}

\subsubsection{Data Filtering and Data Smoothing}

Data filtering and smoothing and sampling will be performed in the following three steps:

(1) Filtering of data sets sharing the same position in the drilling holes. If more than two monitored data sets share the same position, the values of the corresponding monitored drilling parameters (thrust, penetration, torque, and rotation rate) of the first two monitored data sets will be averaged, and the remaining data sets sharing the same position will be filtered out. Usually, for a specific drilling case, the number of data sets can be reduced to half of the original size after data filtering.

(2) Data smoothing. In this research, three data smoothing methods, simple exponential smoothing, double exponential smoothing, and Nadaraya-Watson with Gaussion kernel smoothing will be employed and compared.

(3) Sampling. The definition of sampling is the process by which inference is made to the whole by examining a part ${ }^{[25]}$. From a statistical point of view, the purpose of sampling is to provide various types of statistical information of a qualitative or quantitative nature about the whole by examining a few selected units ${ }^{[25]}$.

The detailed algorithms for data smoothing and sampling techniques follow in this chapter.

\subsubsection{Determination of Rock Properties}

After determining the data filtering and smoothing algorithms, the appropriate drilling parameters and an algorithm by which the locations of interfaces, discontinuities and classification of rock types will be determined and validated in the field. Detailed description of these techniques is in Chapter 4.

\subsection{Data Filter and Smoothing and Sampling}

During the drilling process, the following cases typically happen: 


\section{Research Objectives and Scope and Methodology}

(1) Drilling reaches stall condition (Figure 21). Under this condition, at least two recorded data sets share the same position but other drilling parameters, such as thrust, torque, and rotation rate, may keep changing. The stall condition leads to regrinding and most of the energy will be consumed in friction between the drill bit and rock particles. These resultant, redundant data sets that share the same bit position can seriously misguide the analysis.

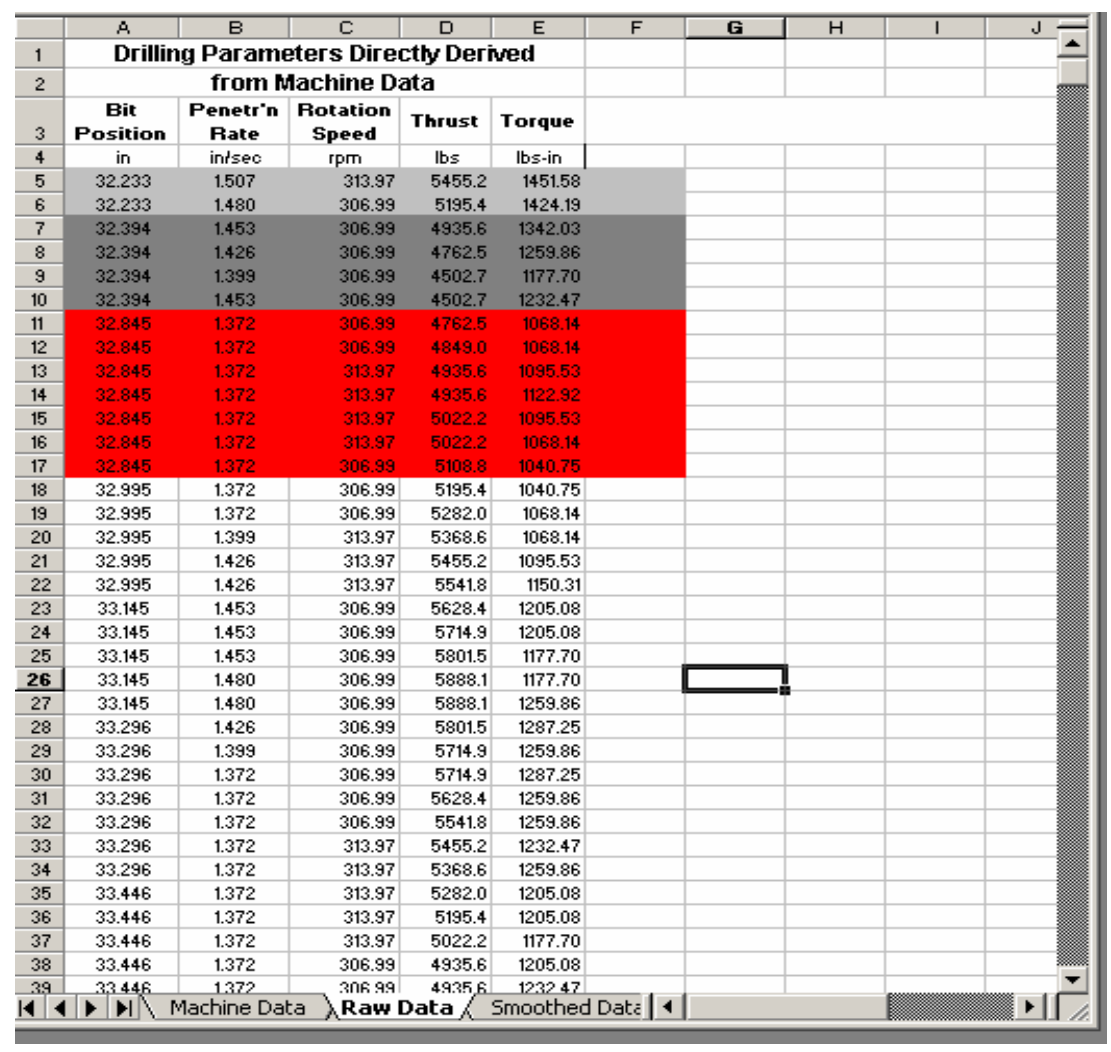

Figure 21 An example of the data sets without filtering (under stall condition)

(2) The physical properties of rock vary since natural rock is generally heterogeneous and anisotropic. Moreover, within the transition zone (the drilling horizon near the interface of two adjacent rock layers), some drilling parameters, such as thrust, may vary considerably. All these phenomena could cause significant variations or unexplainable values for some of the drilling parameters. Therefore, data smoothing technology needs to be employed. 


\section{$\underline{3 \text { Research Objectives and Scope and Methodology }}$}

(3) The fluctuations in drilling parameters often occur when drilling in the same rock layer. Although the major reason for this is assumed to be the ever-changing rock properties, other factors, such as too many data sets in one rock layer, may also cause problems. From a statistical point of view, using fewer data sets that can still accurately reflect the geological information can make the analysis easier and more understandable. For this reason, sampling technology will also be employed to minimize the redundant data sets.

In addition, there are several terms that need to be defined here: (1) Machine data (MD): refers to the data directly recorded by the data acquisition system without any processing. (2) Raw data (RD): refers to the data converted from the machine data in terms of predetermined calibration coefficients. (3) Smoothed data (SD): refers to the data smoothed with certain data smoothing technique. (4) Sampled (smoothed) data (SSD): refers to the data extracted from smoothed data with sampling techniques.

\subsubsection{Filtering of Data Sets Sharing the Same Position}

During the drilling process, the drill will stall if the rock is too strong or excessive thrust is applied. As a result, some data sets can share the same bit position but the corresponding thrust, penetration, torque, and rotation rate are different. In an attempt to reduce and condense the redundant data sets, a data filtering technique is performed on the raw data. This data filtering technique is only applied to the raw data converted from the machine data.

If the number of monitored data sets that share the same position is greater than two, the first two monitored data sets will be averaged, and the remaining data sets sharing the same position will be filtered out. Usually, for a specific drilling case, the number of data sets can be reduced to half of the original size after data filtering. 


\section{Research Objectives and Scope and Methodology}

\subsubsection{Data Smoothing}

For data smoothing, three conservative but very widely accepted data smoothing methods, simple exponential smoothing, double exponential smoothing and NadarayaWatson with Gaussion kernel smoothing were employed and compared.

Single Exponential Smoothing (SES). This smoothing scheme begins by setting $\mathrm{S}_{0}$ to $\mathrm{Y}_{1}$, where $\mathrm{S}$ stands for the smoothed observation, , $\mathrm{Y}$ for the original observation, and the subscripts refer to the data points, $1,2, \ldots, n$. For any data point $t$, the smoothed value $S_{t}$ is found by computing ${ }^{[26]}$

$$
\begin{aligned}
& S_{t}=\alpha \cdot Y_{t}+(1-\alpha) \cdot S_{t-1} \text { or } \\
& S_{t}=\alpha \cdot \sum_{i=0}^{t-1}(1-\alpha)^{i} \cdot Y_{t-i}+(1-\alpha)^{t} \cdot S_{0}
\end{aligned}
$$

Eq.16 was developed due to Roberts (1959) and is called the basic equation of exponential smoothing and the constant $\alpha$ is called the smoothing constant.

The second form of the Eq.16 illustrates the exponential behavior. The weights, $\alpha(1-\alpha)^{t}$ decrease geometrically, and their sum is unity as shown below, using a property of geometric series ${ }^{[26]}$ :

$$
\alpha \sum_{i=0}^{t-1}(1-\alpha)^{i}=\alpha \cdot \frac{1-(1-\alpha)^{t}}{1-(1-\alpha)}=1-(1-\alpha)^{t}
$$

Eq.17 shows that the contribution to the smoothed value becomes less at each consecutive data points. This is the reason why it is called exponential smoothing.

For the single exponential smoothing method, there are two important things to consider. First is the method of setting for $S_{0}$. Usually, $S_{0}$ can be initialized either by $Y_{1}$, setting it equal to the first data point or the average value of the first four or five 


\section{$\underline{3 \text { Research Objectives and Scope and Methodology }}$}

observations. The second item to consider is the determination of the constant $\alpha$. The best value for $\alpha$ is the value which results in the smallest mean square error (MSE) ${ }^{[26]}$. This can be determined using a trial and error approach.

Double Exponential Smoothing(DES). The algorithm for DES can be expressed by Eq.18 ${ }^{[26]}$.

$$
\begin{aligned}
& S_{t}=\alpha \cdot Y_{t}+(1-\alpha)\left(S_{t-1}+b_{t-1}\right) \quad 0<\alpha<1 \\
& \mathrm{~b}_{\mathrm{t}}=\gamma \cdot\left(S_{t}-S_{t-1}\right)+(1-\gamma) \cdot b_{t-1} \quad 0<\gamma<1
\end{aligned}
$$

where $\gamma$ - the trend smoothing constant

There are a variety of schemes used to set initial values for $S_{1}$ and $b_{1}$ in double exponential smoothing. $S_{1}$ is, in general, set to $Y_{1}$ and $b_{1}$ is set to $Y_{2}-Y_{1}$. The best values for $\alpha$ and $\gamma$ are that the values that result in the smallest mean square error (MSE) [26] Again, this can be accomplished using the trial and error approach.

Nadaraya-Watson smoothing Method (NWS). This data smoothing method was originally developed by Nadaraya and Watson in $1964^{[9]}$. The basic algorithm can be expressed as $^{[9]}$

$$
Y_{\text {Smooth }}(j)=\frac{\sum_{i=j-2}^{i=j+2} y_{\text {ori }}(i) \cdot K((D(j)-D(i)) / h)}{\sum_{i=j-2}^{i=j+2} K\left(\frac{D(j)-D(i)}{h}\right)}
$$

where $\mathrm{K}-\mathrm{a}$ function called the kernel. A popular choice of kernel is the Gaussian kernel,

$$
K(x)=\frac{1}{\sqrt{2 \pi}} \exp \left(-\frac{x^{2}}{2}\right)
$$




\section{$\underline{3 \text { Research Objectives and Scope and Methodology }}$}

$\mathrm{h}$ - the bandwidth, $h=1.059 \cdot \sigma \cdot n^{-\frac{1}{5}}$

$\sigma$ - the standard deviation of drilling distance for a specific drilled hole

$\mathrm{n}$ - the number of data sets obtained in one drilled hole

$\mathrm{j}$ - refers to the smoothed data set

$\mathrm{i}$ - refers to the original data set

$\mathrm{D}(\mathrm{i})$ - bit position at point i.

Table 9 and Figures 22, 23 and 24 show the results of an example (using the values of thrust with 340 data sets) comparing the three data smoothing methods. For the single exponential smoothing (SES) method, the best value of $\alpha$ was determined to be 0.95 . The best values of $\alpha$ and $\gamma$ are 0.95 and 0.05 in the double exponential smoothing (DES) method. The Naradaya-Watson method does not need a constant. It can be seen that the SES obtained the minimum mean squared error. Thus the SES method produced the best fit, which means there is much less difference between the smoothed and original data and these points with extremely large or small values can not be extracted from the original data.

Table 9 An example of the comparison with different data smoothing methods (thrust)

\begin{tabular}{|l|c|c|}
\hline Smoothing methods & Constants & MSE* \\
\hline Single exponential (SES) & $\alpha=0.95$ & 506.7 \\
\hline Double exponential (DES) & $\alpha=0.95, \gamma=0.05$ & 99817.5 \\
\hline Naradaya-Watson (NWS) & No constant & 8327.8 \\
\hline
\end{tabular}

*MSE means mean squared error 


\section{Research Objectives and Scope and Methodology}

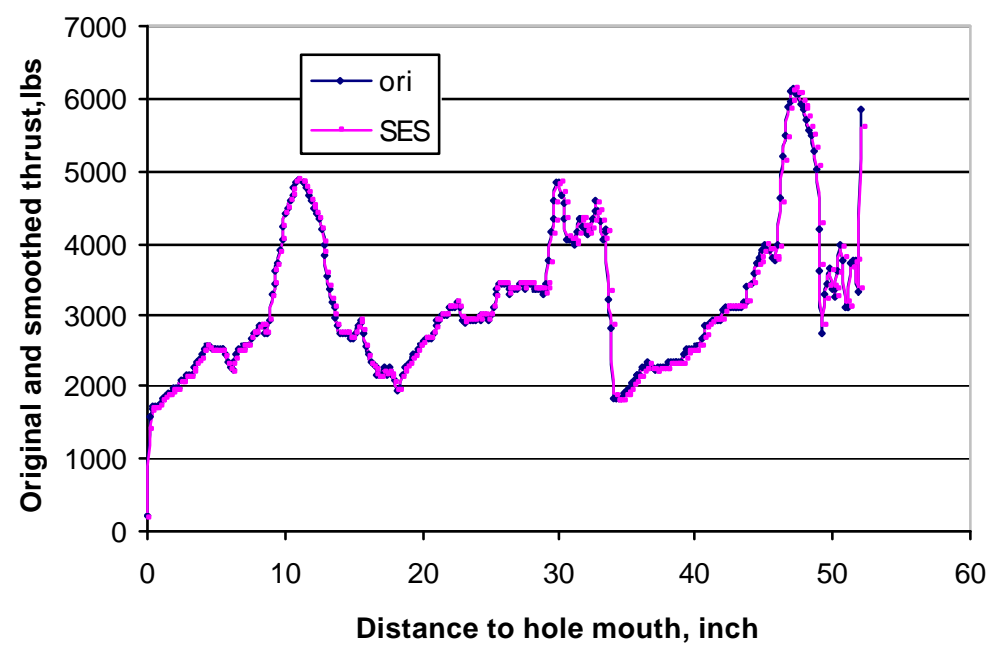

Figure 22 Comparison between original and smoothed data with SES

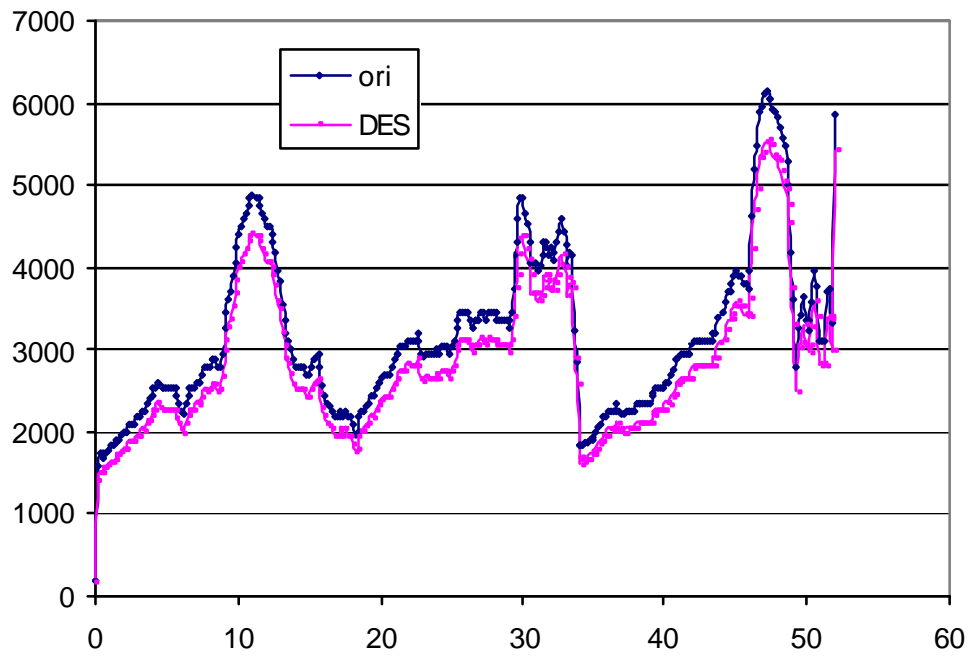

Figure 23 Comparison between original and smoothed data with DES 


\section{Research Objectives and Scope and Methodology}

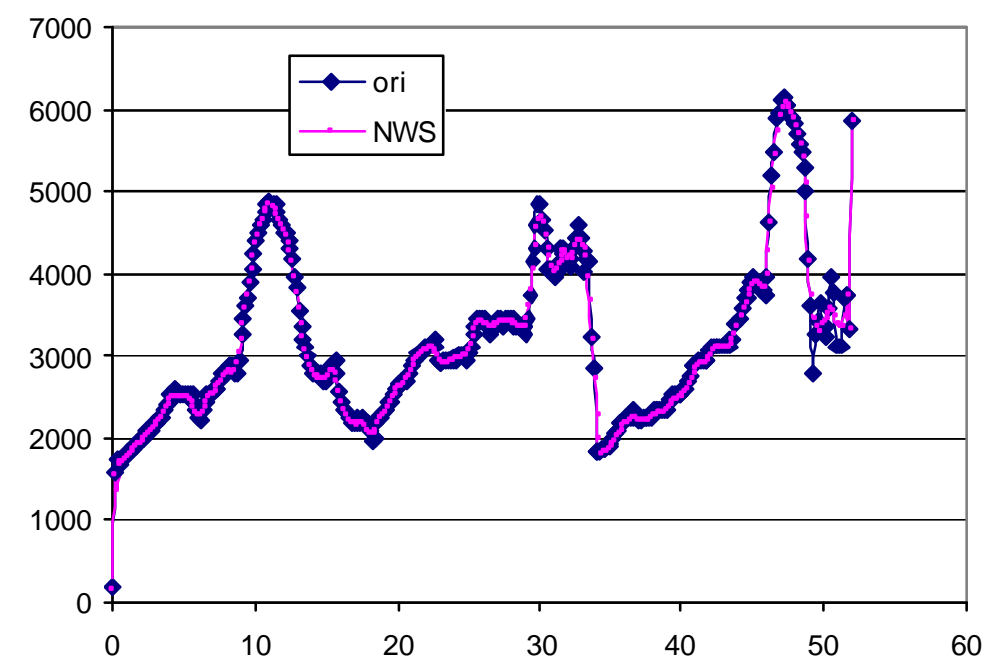

Figure 24 Comparison between original and smoothed data with NWS

The major goal of the data smoothing technique for this specific research is to obtain smoother data and eliminate the magnitude of noise data. In Figure 22, the smoothed data points almost perfectly fit each of the original data points. Thus the SES method did not fit the requirement to reduce noise because it cannot make the original data smoother and eliminate the extremely large values. Despite using the best values of $\alpha$ and $\gamma$, the values of the smoothed data obtained from the DES were smaller than these of the original data (Figure 23), which was the reason why the DES produced the largest MSE. However, SES and DES can be used with sub-optional $\alpha$ and $\gamma$ to smooth better, but it would take more time and judgment on the smoothed data.

Compared with smoothed data obtained from SES and DES, the smoothed data obtained from the NWS seemed smoother and consistent. In addition, it does not need optimal constants $\alpha$ and $\gamma$ for each drilling parameters thereby reduce the complexity of the algorithm of the data smoothing technique.

Based on the analysis above, Naradaya-Watson method (NWS) was chosen as the data smoothing technique used in this specific research. 


\section{Research Objectives and Scope and Methodology}

\subsubsection{Determination of Sampling Method}

From a statistical points of view, sampling is the process by which inference is made of the whole by examining a part ${ }^{[25]}$. Figure 25 shows the basic classification of sampling method $^{[27]}$. In probability samples, each member of the population has a known non-zero probability of being selected. In nonprobability sampling, members are selected from the population in some nonrandom manner. The advantage of probability sampling is that sampling error can be calculated. Sampling error is the degree to which a sample might differ from the population. When inferring to the population, results are reported plus or minus the sampling error. In nonprobability sampling, the degree to which the sample differs from the population remains unknown ${ }^{[27]}$.

$$
\text { Sampling method } \begin{cases}\text { Pr obability }\left\{\begin{array}{l}
\text { Random sampling } \\
\text { Systematic sampling } \\
\text { Stratified sampling }
\end{array}\right. \\
\text { Nonprobabilty }\left\{\begin{array}{l}
\text { Convinence sampling } \\
\text { Judgment sampling } \\
\text { Quota sampling } \\
\text { Snowball sampling }
\end{array}\right.\end{cases}
$$

Figure 25 Classification of sampling methods

In this research the data sets can be sampled periodically, using either the odd sets or the even sets from the hole-data. This periodical sampling method is a type of systematic sampling.

Systematic sampling is often used instead of random sampling. It is also called a Nth name selection technique. After the required sample size has been calculated, every Nth record is selected from a list of population members. As long as the list does not contain any hidden order, this sampling method is as good as the random sampling method. Its 


\section{$\underline{3 \text { Research Objectives and Scope and Methodology }}$}

only advantage over the random sampling technique is simplicity. Systematic sampling is frequently used to select a specified number of records from a computer file.

Table 10 shows the average number of recorded data sets within a one-inch horizon for four drilled rock layers (Block 6, layered block). The laboratory test was performed in the spring of 2002. The numerator in each table cell is the total recorded number of data sets for the corresponding rock layer in each hole, and the denominator is the thickness of the corresponding rock layer in each hole. Theoretically, the number of data sets per unit distance should decrease with the increase of penetration rate, but the anisotropy and the delayed adjustment of the hydraulic system complicate the drilling process, which brings the data sets to a various number even with the same controlled drilling parameters.

Table 10 The average number of recorded data sets within one-inch horizon of rock layers, Block 6 (Layered block)

\begin{tabular}{|c|c|c|c|c|}
\hline Hole No. and pre-setting & $\begin{array}{l}\text { Light brown } \\
\text { sandstone }\end{array}$ & Argillite & White marble & Concrete \\
\hline $1, \mathrm{pr}^{*}=1.1$, rotation rate $=300$ & $44 / 7=6.29$ & $42 / 6.5=6.46$ & $31 / 5=6.2$ & $74 / 11=6.73$ \\
\hline $2, \mathrm{pr}=1.5$, rotation rate $=300$ & $45 / 7=6.43$ & $45 / 6.5=6.92$ & $31 / 5=6.2$ & $68 / 11=6.18$ \\
\hline $3, \mathrm{pr}=1.5$, rotation rate $=300$ & $40 / 7=5.71$ & $39 / 6.5=6$ & $12 / 2=6$ & $69 / 11=6.27$ \\
\hline $4, \mathrm{pr}=1.5$, rotation rate $=300$ & $40 / 7=5.71$ & $38 / 6.5=5.85$ & $14 / 2.2=6.36$ & $59 / 11=5.36$ \\
\hline $5, \mathrm{pr}=1.1$, rotation rate free & $38 / 7=5.29$ & $35 / 6.5=5.39$ & $32 / 5=6.4$ & $63 / 11=5.73$ \\
\hline $6, \mathrm{pr}=0.6$, rotation rate free & $38 / 7=5.42$ & $40 / 6.5=6.15$ & $28 / 4.5=6.22$ & $73 / 11=6.64$ \\
\hline $7, \mathrm{pr}$ free, rotation rate $=400$ & $39 / 7=5.57$ & $42 / 6.5=6.46$ & $33 / 5.5=5$ & $41 / 11=3.73$ \\
\hline $8, \mathrm{pr}$ free, rotation rate $=500$ & $38 / 7=5.43$ & $37 / 6.5=5.69$ & $24 / 4=6$ & $33 / 11=3$ \\
\hline Average & 5.73 & 6.115 & 6.05 & 5.46 \\
\hline
\end{tabular}

Notes: $p r=$ penetration rate

Table 10 shows that there is a consistent number of recorded data sets within each oneinch horizon of each rock type in the eight drilled holes. On average, the number of recorded data sets is six per one-inch section, which means, there are about 6 data sets which can be recorded and the distance between two data sets is about 0.1667 inch (4.233 


\section{Research Objectives and Scope and Methodology}

$\mathrm{mm})$. If three data sets are chosen periodically within a one-inch horizon, the distance between samples is about 0.3333 inch $(8.4667 \mathrm{~mm})$, which is acceptable because it can still ensure that few data points were missed. However, in an attempt to make the determination of locations of discontinuities more accurate, the SD (smoothed data) other than the SDD (smoothed and sampled) is suggested to use. The sampled data is only used in discriminant analysis.

Figure 26 shows an example of the data sets (Hole No.3, thrust) before and after sampling. It can be seen that the SSD fitted the SD very well and did not change the variation trend. In addition, there is no significant difference (except that the variance after sampling is a little bit larger than that before sampling) between the standard deviation before and after sampling (less than 2.85\%) (Table 11), which implies the sampled data are reliable.

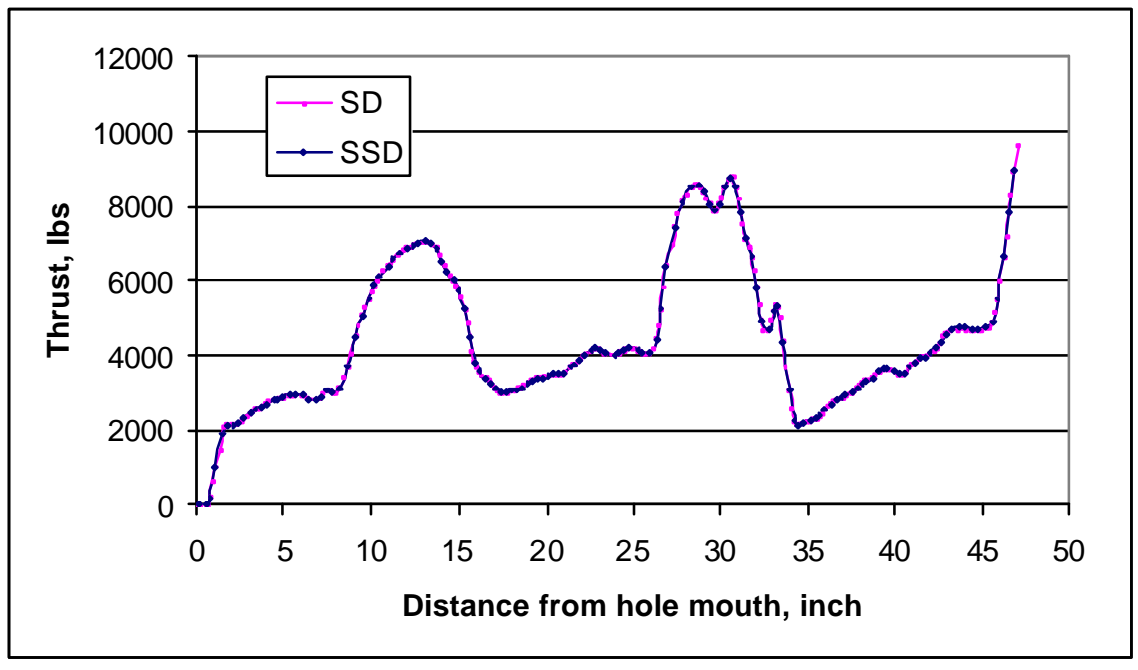

Figure 26 Comparison between SD (smoothed data) and SSD (smoothed and sampled data), Hole No. 3, Block 6 


\section{$\underline{3 \text { Research Objectives and Scope and Methodology }}$}

Table 11 Comparison of standard deviation before and after sampling (thrust, for smoothed data), Block 6

\begin{tabular}{|c|c|c|c|c|c|c|c|c|}
\hline Hole No. & 1 & 2 & 3 & 4 & 5 & 6 & 7 & 8 \\
\hline Before sampling & 1075.4 & 981 & 1855.3 & 1594 & 2007 & 1440 & 1067.5 & 1213 \\
\hline After sampling & 1067.8 & 959 & 1802.4 & 1590 & 2005.4 & 1437 & 1055 & 1202 \\
\hline Difference & 7.6 & 22 & 52.9 & 4 & 1.6 & -3 & 12.5 & 11 \\
\hline
\end{tabular}

It must be pointed out that the smoothed and sampled data (SSD) are only used to classify the rock type, but the smoothed data without samples are used to determine the locations of discontinuities.

\subsubsection{Summary of Data Filter and Data Smoothing}

Data filtering and smoothing are indispensable in this research. From Table 12 it can be seen that, after data filtering, smoothing and sampling, the reduction percentage, which is the ratio of the reduced number to raw number, is very high up to $79.9 \%$, and most of them are larger than $60 \%$. This is a significant improvement to the raw data and makes the analysis of the drilling data much easier.

Table 12 Reduction percentage of data sets after data filtering and smoothing and sampling, Block 6

\begin{tabular}{|c|c|c|c|c|c|c|c|c|}
\hline Hole No. & 1 & 2 & 3 & 4 & 5 & 6 & 7 & 8 \\
\hline Hole length, in & 52 & 52 & 47 & 47.2 & 50 & 49.5 & 50.5 & 49 \\
\hline Number of RD & 521 & 836 & 440 & 366 & 425 & 676 & 438 & 302 \\
\hline Number of SD & 337 & 337 & 289 & 274 & 297 & 311 & 264 & 221 \\
\hline Number of SSD & 168 & 168 & 144 & 137 & 148 & 156 & 232 & 111 \\
\hline $\begin{array}{c}\text { Reduction } \\
\text { percentage (\%) }\end{array}$ & $\mathbf{6 7 . 7 6}$ & $\mathbf{7 9 . 9}$ & $\mathbf{6 7 . 2 7}$ & $\mathbf{6 2 . 5 7}$ & $\mathbf{6 5 . 1 8}$ & $\mathbf{7 6 . 9}$ & $\mathbf{4 7 . 0 3}$ & $\mathbf{6 3 . 2 5}$ \\
\hline
\end{tabular}




\section{Development of Algorithms for Determination of Rock Properties}

Theoretically, drilling is a process that transfers mechanical energy to rock in the form of thrust and/or torque to break and overcome resistance from the drilled rock. The energy required to excavate a given volume of rock is called specific energy as defined

by Teale ${ }^{[2]}$, which should entirely depend on the properties of the drilled rock. However, the calculation of specific energy is achieved by using the directly monitored mechanical drilling parameters (Eq.10) that content a lot of uncontrolled noise components due to the adjustment of the hydraulic system and the discrepancies of the data logging system. When drilling near the discontinuities, the variation in thrust and torque will be reflected directly in the readings of penetration rate and rotation rate even when the penetration rate or rotation rate are tried to be controlled as constants. All the factors during the drilling process will inevitably produce fluctuations in the calculated specific energy. This indicates that not all the fluctuations are caused by the variation of rock properties but also some of them come from the adjustment of the hydraulic system. As a result, the amount of the required energy to excavate a volume of rock is different from the mechanical energy obtained from the calculation, although researchers try to use the latter to represent the former. Some of the difference between the calculated mechanical energy and the actual energy consumed in drilling the rock is energy losses in dissipations in friction, grinding, and torsion of drill rod, etc. during the interaction of rock-drill.

During the drilling process, some of the applied mechanical energy is used to move the roof bolter head up with a certain speed (in the form of kinetic and potential energies), and some of the energy is expended on rod-to-rock transmission in the form of torsion energy. The remaining energy is needed to overcome frictional resistance between the front of the bit and rock, and break the cementing bond of the drilled rock. These energy losses that do not contribute to rock, such as kinetic, potential, torsion, and thermal energies, will influence the values of the recorded drilling parameters and make thrust, 


\section{Development of Algorithms for Determination of Rock Properties}

torque and penetration rate either higher or lower, especially in the transition zone where the drill bit is near the interface of two adjacent rock layers. The algorithms for determining rock properties were developed in this chapter by considering those factors above.

In order to determine rock properties using the recorded drilling parameters, a new drilling parameter called drilling hardness $(\mathbf{D H})$ was derived. The drilling hardness demonstrates the relative rock strength and resistance to indentation to the drill bit under an applied stress. While drilling through a weak area or discontinuities or interface, the value of drilling hardness should be lower, and while drilling in harder rock, it should be higher. This parameter is proposed to determine the locations of discontinuities such as the interfaces of rock layers, and fractures in the rock.

In order to be useful for roof mapping, a derived drilling parameter should meet the following requirements: (1) It should be derived from the five original drilling parameters, thrust, penetration rate, torque, rotation rate, and bit position. (2) It should consider the energy lost to the environment during the drilling process. (3) It should, to a certain degree, reflect the differences while drilling different rock layers and maintain consistency while drilling within the same rock layer.

In this chapter, drilling hardness was derived from the drilling mechanical model (DMM) and the basic algorithms were developed mainly based on the laboratory tests conducted in Jan., 2002 on Block 6 (Figure 27) and the laboratory testing parameters are shown in Table 13. To alleviate concerns of decreasing bit sharpness during drilling, each hole was drilled by using a new bit. The validation of utilizing drilling hardness for the determination of rock properties is accomplished by the field tests. 


\section{Development of Algorithms for Determination of Rock Properties}

Table 13 Parameters used in laboratory testing on Block 6

\begin{tabular}{|c|c|c|c|c|c|}
\hline Hole No. & Bit size & $\begin{array}{c}\text { P.R } \\
\text { in/sec }\end{array}$ & $\begin{array}{c}\text { rotation rate } \\
\text { Rev./min. }\end{array}$ & $\begin{array}{c}\text { Thrust cap } \\
\text { psi }\end{array}$ & $\begin{array}{c}\text { Hole Length } \\
\text { in }\end{array}$ \\
\hline 1 & $1-1 / 32$-in & 1.1 & 300 & 707 & 52.0 \\
\hline 2 & $1-1 / 32$-in & 1.5 & 300 & 707 & 52.0 \\
\hline 3 & $1-3 / 8$-in & 1.5 & 300 & 1,132 & 48.5 \\
\hline 4 & $1-3 / 8$-in & 1.5 & 300 & 1,132 & 47.0 \\
\hline 5 & $1-3 / 8$-in & 1.1 & Free & 1,000 & 49.5 \\
\hline 6 & $1-3 / 8$-in & 0.6 & Free & 1,000 & 49.5 \\
\hline 7 & $1-3 / 8$-in & free & 400 & 1,000 & 50.0 \\
\hline 8 & $1-3 / 8$-in & free & 500 & 1,000 & 49.0 \\
\hline
\end{tabular}
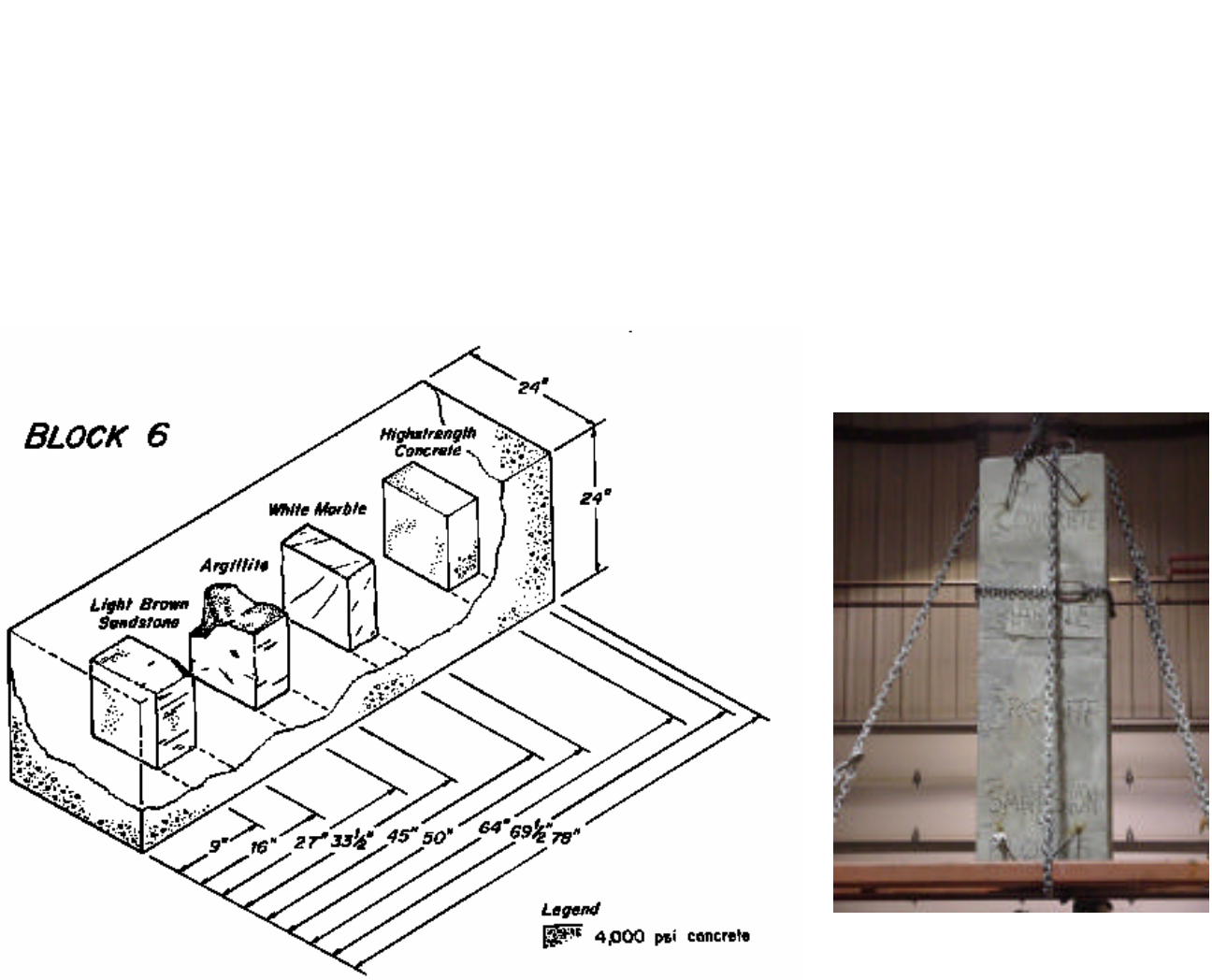

Figure 27 The layered block (Block 6) drilled in laboratory 


\section{Development of Algorithms for Determination of Rock Properties}

\subsection{Determination of a New Drilling Parameter - Drilling Hardness (DH)}

The determination of the new drilling parameter (DH) includes three parts, determination of the frictional coefficient between the drill bit and rock, determination of the contact area between the front part of the drill bit and rock, and determination of the new drilling parameter. The algorithms, including data filtering and smoothing, calculation of frictional coefficient, contact area, drilling hardness, and slope, are presented in Appendix I.

\subsubsection{Determination of the Frictional Coefficient}

During the drilling process, as discussed above, the friction between the drill bit and rock plays an important role in energy consumption. However, the calculated friction coefficients at different drilling depths in a single drill hole will be different because the abrasion and comminution of the rock surface result in variable resistance due to the irregular shearing of asperities and granular structure of rock (see Section 4.3). Therefore, the calculation of the frictional coefficient should be applied to each data set.

The determination of the frictional coefficient between the drill bit and rock is based on such an assumption that the physical properties of the drill bit do not change during the drilling process. Therefore the calculated frictional coefficient in the axial and rotation directions should be identical. The calculated frictional coefficient was used as one of the parameters in determining the drilling hardness.

Since the drilling is a dynamic process which relates to movements in axial and rotational directions, the principle of energy equilibrium can be applied. Equation 20 was developed based on the principle of energy equilibrium and the assumption that the (residual) shear strength of the rock is not taken into account since the rock underneath the drill bit with certain depth will be yielded or broken after the drill bit indents into the rock. The work done by the torque mainly comprises of three parts. The first part is the 


\section{Development of Algorithms for Determination of Rock Properties}

change in the kinetic energy $\left(I \omega_{i}^{2} / 2-I \omega_{i-1}{ }^{2} / 2\right)$, the second part is the energy to overcome the friction resistance of rock in the tangential direction $\left(\mathrm{Fi} \cdot \mathrm{f}_{\mathrm{i}} \cdot \mathrm{R} \cdot \theta_{\mathrm{i}}\right)$, and the third part is the torsion energy $\left(\tau_{\mathrm{i}}^{2} \cdot \mathrm{L} / \mathrm{G} \cdot \mathrm{I}_{\mathrm{p}}\right)$. In this model, $\mathrm{r}_{1}$ and $\mathrm{r}_{2}$ denote the inner and outer radii respectively since both of them are used in calculating the torsion energy consumed in the drill rod. $\mathrm{R}$ denotes the outer radius of the drill bit since friction occurs between the bit and rock. This energy relationship can be expressed by

$$
\left\{\begin{array}{l}
\tau_{i} \cdot \theta_{i}=\frac{1}{2} I \cdot \omega_{i}{ }^{2}-\frac{1}{2} I \cdot \omega_{i-1}{ }^{2}+F_{i} \cdot f_{i} \cdot R \cdot \theta_{i}+\frac{\tau_{i}{ }^{2} \cdot L}{G \cdot I_{p}} \\
\Rightarrow f_{i}=\frac{\tau_{i} \cdot \theta_{i}-\frac{1}{2} I \cdot \omega_{i}{ }^{2}+\frac{1}{2} I \cdot \omega_{i-1}{ }^{2}-\frac{\tau_{i}{ }^{2} \cdot L}{G \cdot I_{p}}}{F_{i} \cdot R \cdot \theta_{i}}
\end{array}\right.
$$

where $f_{i}-$ the frictional coefficient at point $i$, derived from Eq.20

$\theta_{\mathrm{i}}$ - rotational angle from point $\mathrm{i} 1$ to i, $\theta=\omega_{i-1} \cdot t+\frac{1}{2} a_{i} \cdot t^{2}$

$\mathrm{L}$ - length of the drill rod, 62 inch

$\mathrm{G}$ - shear modulus of the rod, $11.5 \times 10^{6}, \mathrm{lb} / \mathrm{in}^{2}$

I - mass moment of inertia of drill rod and bit along the rotation axis

$$
I=0.083 \cdot m\left(r_{1}^{2}+r_{2}^{2}\right), \mathrm{lb}-\mathrm{in}^{2}
$$

$\mathrm{m}$ - mass of the rod, $\mathrm{lbm}$

Ip - moment of inertia of drill rod along the rotation axis

$$
I_{p}=1.9246\left(r_{2}^{4}-r_{1}^{4}\right)
$$

$\mathrm{r}_{1}, \mathrm{r}_{2}-$ the inner and outer radii of the drill rod, inch

$\mathrm{R}$ - the outer radius of the drill bit, inch

$\tau_{\mathrm{i}}$ - torque measured at point I, lb-in

The mass moment of inertia of the drill rod with hexagon cross-section is derived based on the definition of mass moment of inertia. The density of the rod is 


\section{Development of Algorithms for Determination of Rock Properties}

$$
\rho=\frac{m}{3.4641\left(r_{2}^{2}-r_{1}^{2}\right) \cdot L}
$$

The mass moment of inertia of the drill is derived as

$$
I=\iint x^{2} \cdot \rho \cdot d V=\iint x^{2} \cdot \rho \cdot \tan 30 \cdot x \cdot 2 \cdot d x \cdot d x=\int_{0}^{L} d z \int_{r_{1}}^{r_{2}} 1.1547 \cdot \rho \cdot d x^{4}=0.083 \cdot m\left(r_{1}^{2}+r_{2}^{2}\right)
$$

Figure 28 shows a typical example (Hole No.5, Block 6) of the calculated frictional coefficient derived by Eq.20. It can be seen that while drilling in different layers the frictional coefficients are little different. When drilling in concrete layer, the calculated frictional coefficient becomes smaller. The advantage of using Eq. 20 to calculate the frictional coefficient is that it does not need to consider the contact area between the drill bit and rock.

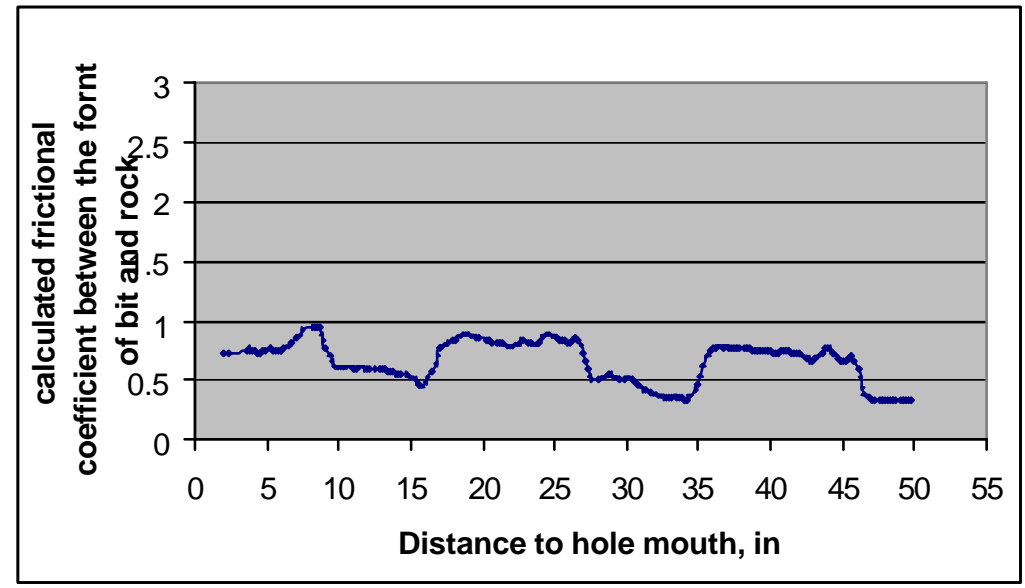

Figure 28 Calculated frictional coefficients along a drilled hole

\subsubsection{Determination of Contact Area in Axial Direction}

The contact area $A_{i}$ refers to the area between the front part of the bit and the corresponding rock while the drill bit is at point $\mathrm{i}$. It is a function of the bite-depth per 


\section{Development of Algorithms for Determination of Rock Properties}

revolution. According to the geometry of the drill bit (1-3/8-in drill bit) shown in Figure 29 , the contact area can be estimated by measuring the dimension of the contacts between the drill bit and rock (the contact part is a combination of triangles and squares). The estimated contact area can be expressed by

$A_{i}=\left\{\begin{array}{l}1.1518 \cdot p_{-} r_{i}, \text { if } p_{-} r_{i} \leq 0.125^{\prime \prime} \\ 1.1518 \cdot p_{-} r_{i}+18.04762 \cdot\left(p_{-} r_{i}-0.125\right)^{2}, \text { if } 0.28125^{\prime \prime} \geq p_{-} r_{i} \geq 0.125^{\prime \prime}\end{array}\right.$

where, $p_{-} r_{i}-$ bite depth per revolution, in/rev.

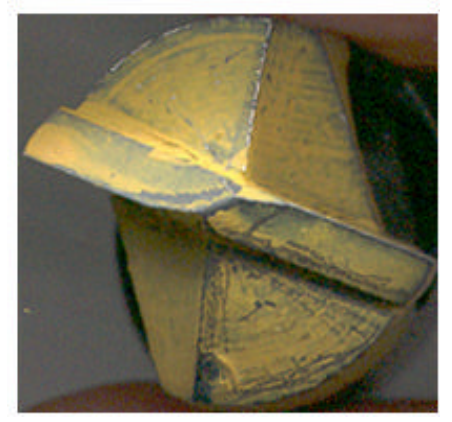

Figure 29 Front part of a 1-3/8-in drill bit

For the 1-1/32-in drill bit, the contact area can be estimated by

$$
A_{i}=\left\{\begin{array}{l}
1.0607 \cdot p_{-} r_{i}, \text { if } \quad p_{-} r_{i} \leq 0.125^{\prime \prime} \\
0.13258+1.32583 \cdot\left(p_{-} r_{i}-0.125\right), \text { if } 0.28125^{\prime \prime} \geq p_{-} r_{i} \geq 0.125^{\prime \prime}
\end{array}\right.
$$

\subsubsection{Drilling Mechanical Model (DMM) and Determination of the Drilling Hardness}

The drilling mechanical model (DMM) (Figure 30), adopted to determine the drilling hardness, is based on the following conditions:

(1) During the period of drilling from point i-1 to i, the work done by thrust includes four parts: kinetic energy, potential energy, work done to overcome friction 


\section{Development of Algorithms for Determination of Rock Properties}

resistance in the axial directions, and work done to overcome the resistance from rock by breaking the cementing bond of or penetrating into the drilled rock (Here, the stress used to break the cementing bond of or penetrate into the drilled rock is named drilling hardness, which reflects the resistance to indentation to the drill bit).

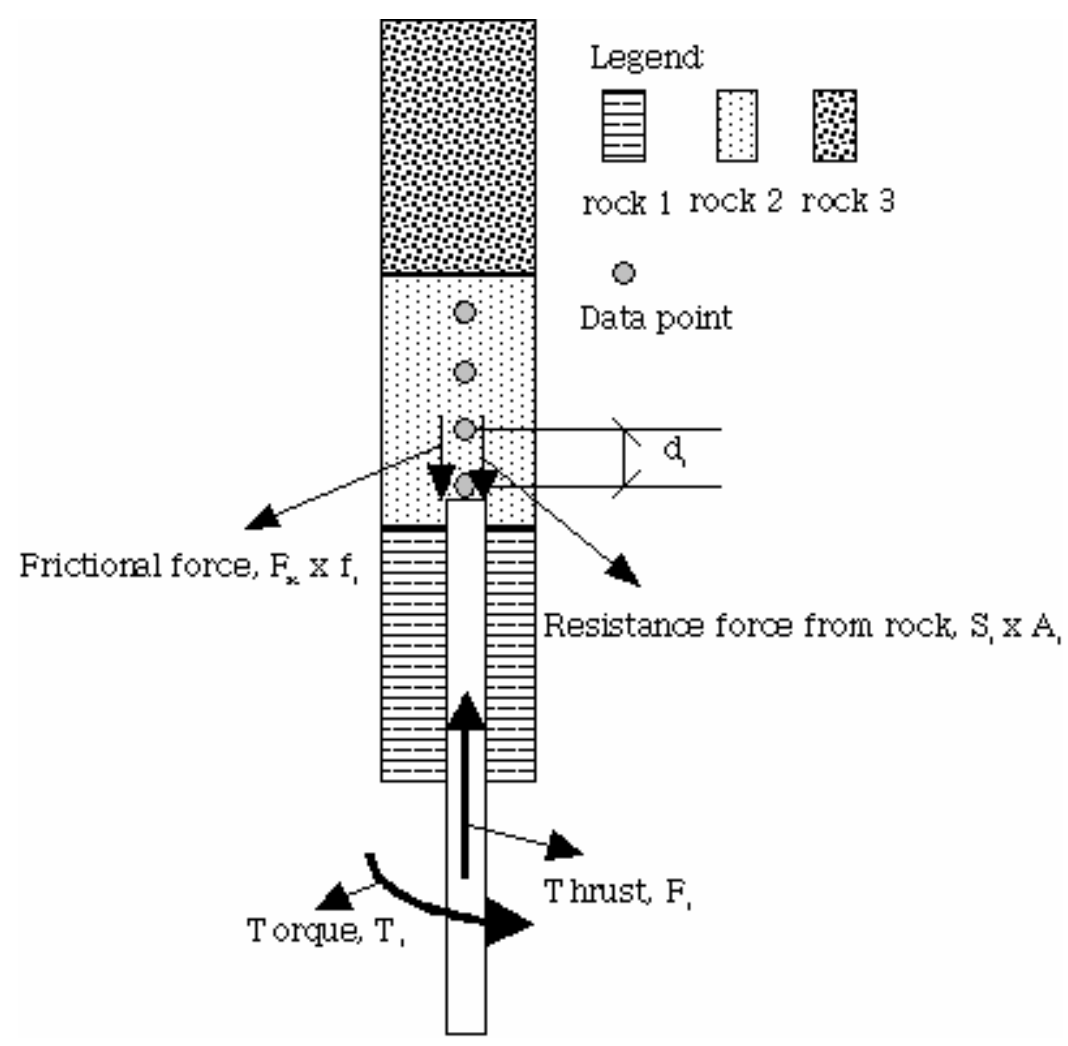

Figure 30 Schematic of the force components in drilling operation

(2) During the period of drilling, the bit keeps rotating so that bit position keeps changing, and the required thrust and torque and frictional coefficient $f_{i}$ and the contact area $A_{i}$ vary, because the strength of the cementing bond and/or particles in contact with the drill bit varies even within one type of rock. The frictional coefficient between the bit and the drilled rock was calculated by Eq. 20 and the contact area was calculated by Eq.21 for a 1-3/8-in bit. The contact area $A_{i}$ is assumed no-change while drilling from point $\mathrm{i}-1$ to $\mathrm{i}$. 


\section{Development of Algorithms for Determination of Rock Properties}

(3) The frictional force between the front of the bit and rock in the axial direction was calculated by using the simplified load disintegration analysis (Figure 31). This model is used to determine the force $\left(\mathrm{F}_{\mathrm{x}}\right)$ perpendicular to the drilling direction. Here $\mathrm{c}$ is a factor by considering the geometry of the bit (Figure 31) and values $71^{\circ}$ and $19^{\circ}$ were measured based on the geometry of the drill bit $\left(\mathrm{F}_{\mathrm{x}}=\mathrm{c} \times \mathrm{F}\right.$, and $\left.\left.\mathrm{c}=\cos 19 \times \sin 19=0.3078\right)\right)$.

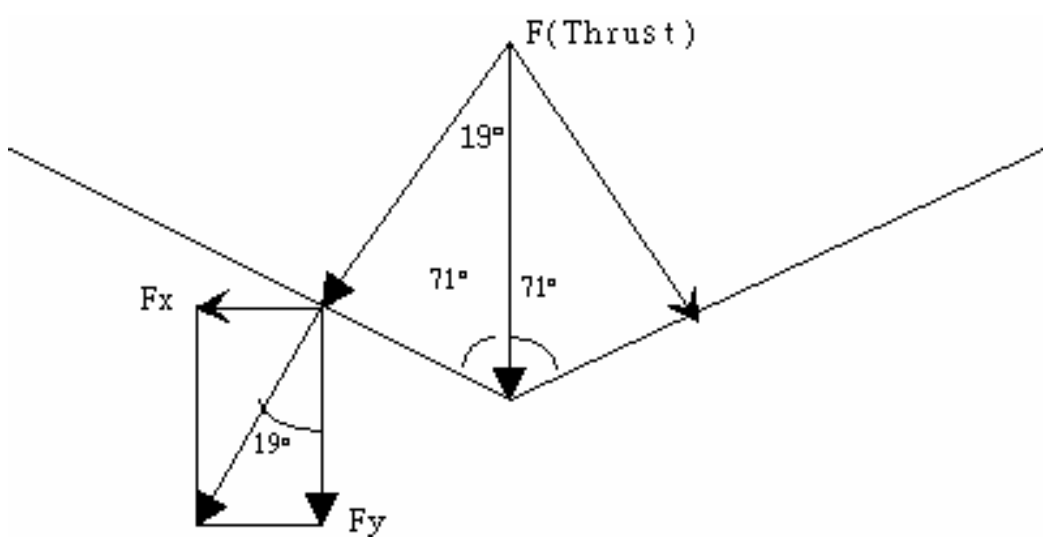

Figure 31 Simplified schematic of thrust force component

Based on the principle of energy equilibrium, the drilling mechanical model (DMM) and expression reflecting the relationship ${ }^{[28]}$ between the work done by thrust and the energy losses in the drilling direction is written as

$$
F_{i} \cdot d_{i}=\frac{1}{2} m \cdot\left(\left(p r_{i}\right)^{2}-\left(p r_{i-1}\right)^{2}\right)+c \cdot F_{i} \cdot f_{i} \cdot d_{i}+m g \cdot d_{i}+S_{i} \cdot A_{i} \cdot d_{i}
$$

where $\mathrm{S}_{\mathrm{i}}-$ calculated drilling hardness (the new drilling parameter) at position $\mathrm{i}$, psi

$F_{i}-$ measured thrust at point $\mathrm{i}, \mathrm{lbs}$

$\mathrm{m}$ - mass of the drill bit, rod, mast and roof header

$\mathrm{pr}_{\mathrm{i}}-$ measured penetration rate at position $\mathrm{i}, \mathrm{in} / \mathrm{sec}$

$\mathrm{d}_{\mathrm{i}}-$ distance drilled from point $\mathrm{i}-1$ to $\mathrm{i}$

$A_{i}-$ contact area between the bit and the drilled rock, in ${ }^{2}$ 


\section{Development of Algorithms for Determination of Rock Properties}

$f_{i}-$ frictional coefficient between the front part of the drill bit and rock at position $\mathrm{i}$

$F_{i} \cdot d_{i}$ - work done by thrust from point $\mathrm{i}-1$ to $\mathrm{i}$

$\frac{1}{2} m \cdot\left(\left(p r_{i}\right)^{2}-\left(p r_{i-1}\right)^{2}\right)-$ change of kinetic energy from point $\mathrm{i}-1$ to point $\mathrm{i}$

$c \cdot F_{i} \cdot f_{i} \cdot d_{i}$ - work used to overcome the frictional resistance in drilling (axial) direction

$m g \cdot d_{i}$ - potential energy from point $\mathrm{i} 1$ to $\mathrm{i}$

$S_{i} \cdot A_{i} \cdot d_{i}$ - work used to overcome the resistance of rock

Thus, the drilling hardness $S_{\mathrm{i}}$ is

$$
S_{i}=\frac{F_{i} \cdot d_{i}\left(1-c \cdot f_{i}\right)-0.5 \cdot m \cdot\left(\left(p r_{i}\right)^{2}-\left(p r_{i-1}\right)^{2}\right)-m g \cdot d_{i}}{d_{i} \cdot A_{i}}
$$

Figure 32 shows an example (Hole 4, Block 6) of the calculated drilling hardness (DH) along one drilled hole using Eqs. 20, 21, and 24

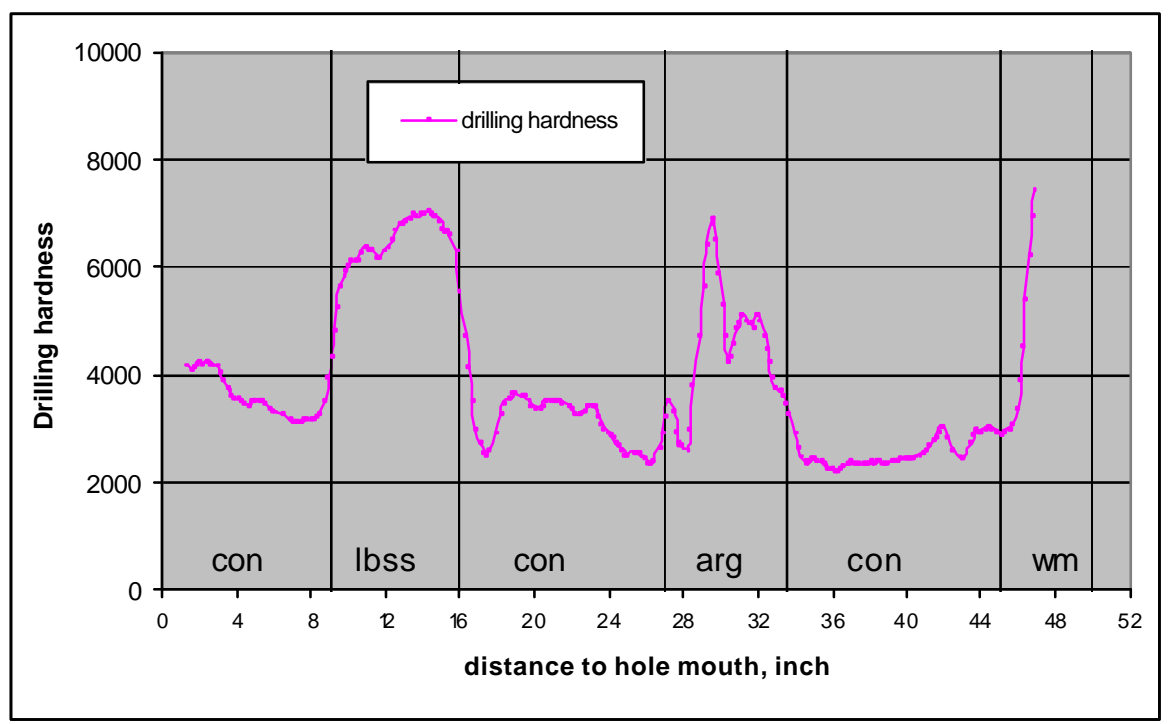

Figure 32 An example of the drilling hardness (Hole 4, Block 6) 


\section{Development of Algorithms for Determination of Rock Properties}

It can be seen that near the interfaces between rock layers, there is a large jump or drop in the drilling hardness. This parameter, DH, satisfies the requirements of a derived drilling parameter mentioned previously. It is derived from the original drilling parameters by considering the energy lost in the drilling and rotational directions, and reflects the differences when drilling in different rock layers, although there are still some fluctuations within the same rock layer. In addition, it can been inferred from the distributions of the drilling hardness and its slope that while drilling from soft to hard rock (e.g. drilling from concrete to light brown sandstone) the slope varies upward and while drilling from hard to soft rock (e.g. drilling from argillite to concrete) the slope varies downward. Basically, drilling hardness shows some consistency of rock resistance while drilling within one rock layer and more pronounced variation of rock resistance between rock layers. The variation of DH while drilling in different rocks can be used to determine the locations of the discontinuities. In general, the stronger the drilled rock, the higher the value of $\mathrm{DH}$.

\subsection{Determination of the Locations of Interfaces and Discontinuities}

Although the drilling hardness shows the differences in magnitude for each type of rock, it is still hard to determine the locations of interfaces between rock layers. There are still a lot of fluctuations for some rock layers, although those differences in magnitude within the same rock layer are not as large as those near the interfaces between rock layers. In order to make it easier to detect the variation features in drilling hardness and determine the locations of interfaces, the slope of the drilling hardness is determined. The definition of the slope of the curve for the drilling hardness is the vertical distance divided by the horizontal distance between any two points on the curve, which is the rate of change along the regression line. The equation for the slope of the regression line is 


$$
\text { slope }(i)=\frac{n \cdot \sum_{j=1}^{n} x_{j} \cdot y_{j}-\left(\sum_{j=1}^{n} x_{j}\right)\left(\sum_{j=1}^{n} y_{j}\right)}{n \sum_{j=1}^{n} x_{j}{ }^{2}-\left(\sum_{j=1}^{n} x_{j}\right)^{2}}
$$

where slope(i) - the slope at point i

$\mathrm{n}$ - the number of data points used to calculate the slope at point $\mathrm{i}$

$\mathrm{x}_{\mathrm{j}}$ - the drilling distance from the hole mouth at point $\mathrm{i}-\mathrm{j}$ or $\mathrm{i}+\mathrm{j}$

$y_{j}-$ the value of drilling hardness at point $i-j$ or $i+j$

After determining the slope of the calculated drilling hardness, the locations of interfaces were determined by establishing a threshold value from the slope and finding the outliers of the slope. The procedure of determining the outliers is as follows

(1) Arrange the data sets of slope in order of magnitude;

(2) Pick the lower quartile, $Q_{1}$, which is the value of the slope in the position $0.25(\mathrm{i}+1)$. Then pick the upper quartile, $\mathrm{Q}_{3}$, which is the value of the slope in the position $0.75(\mathrm{i}+1)$. When these positions are not integers, using the average values in the two adjacent positions;

(3) Calculate the inter-quartile range (IQR). IQR is the difference between the upper and lower quartiles, that is, $\mathrm{IQR}=\mathrm{Q}_{3}-\mathrm{Q}_{1}$.

(4) Calculate the inner and outer fences as follows:

Inner fences: $\mathrm{Q}_{1}-1.5 \times(\mathrm{IQR})$ and $\mathrm{Q}_{3}+1.5 \times(\mathrm{IQR})$

Outer fences: $\mathrm{Q}_{1}-3 \times(\mathrm{IQR})$ and $\mathrm{Q}_{3}+3 \times(\mathrm{IQR})$

(5) Determine the outliers.

Suspect outliers: $\mathrm{Q}_{3}+1.5 \times(\mathrm{IQR}) \leq$ slope $\leq \mathrm{Q}_{3}+3 \times(\mathrm{IQR})$ or

$$
\mathrm{Q}_{1}-1.5 \times(\mathrm{IQR}) \geq \text { slope } \geq \mathrm{Q}_{1}-3 \times(\mathrm{IQR})
$$

Extreme outliers: slope $\geq \mathrm{Q}_{3}+3 \times(\mathrm{IQR})$ or

$$
\text { slope } \leq \mathrm{Q}_{1}-3 \times(\mathrm{IQR})
$$




\section{Development of Algorithms for Determination of Rock Properties}

The following set of data shows an example of how to determine the locations of interfaces of rock layers. By following the five steps for determining the outliers, all the required parameters are obtained:

$$
\begin{aligned}
& \mathrm{Q} 1=-300.09 \text { and } \mathrm{Q} 3=349.2604 \\
& \mathrm{IQR}=\mathrm{Q} 3-\mathrm{Q} 1=349.2604-(-300.09)=649.3504
\end{aligned}
$$

Inner fences: $\mathrm{Q}_{1}-1.5 \times(\mathrm{IQR})=-1274.1156$ and $\mathrm{Q}_{3}+1.5 \times(\mathrm{IQR})=1323.286$

Outer fences: $\mathrm{Q}_{1}-3 \times(\mathrm{IQR})=-2248.1412$ and $\mathrm{Q}_{3}+3 \times(\mathrm{IQR})=2297.3116$

Therefore in this case, it can be concluded that it is an extreme outlier if the slope is less than -2248.1412 or larger than 2297.3116. If the slope at the location $i$ is within the range [-2248.1412, -1274.1156] or [1323.286, 2297.3116], it can be considered as a suspect outlier. This method will be further improved by the following laboratory and field tests.

The basic idea of using these outliers either above the outer fences or between the outer and inner fences is to determine the locations of interfaces between rock layers and discontinuities within one rock layer. In the following sections, the term discontinuity is used to specify the lack of continuity within one rock layer, such as fractures, cracks, etc. The criteria used in identifying an interface and a discontinuity are determined in the following three steps:

(1) Apply the basic criteria to the slope of drilling hardness for a drilled hole. The basic criteria are that if the value of the slope at location y is larger than or equal to that of the outer fence, then y will be identified as the location of an interface. In addition, Figure 33 shows that there are at least one point near the interface whose value(s) of slope is (are) beyond the outer fence. Thus, the location of the peak value from these points whose values of slope are beyond the outer fence is determined as the location of the interface. If the peak value of the negative slope at location $\mathrm{y}$ is between the outer and inner fences, then $\mathrm{y}$ will be identified as the location of a discontinuity, regardless it is a fracture or a crack as observed from the scope or core log. The reason why only the negative slope is used in 


\section{Development of Algorithms for Determination of Rock Properties}

determining the location of discontinuities is that these discontinuities are weak in strength. When drilling through a discontinuity, usually the corresponding drilling hardness tends to decrease; thus the slope of the drilling hardness around that location is negative. The positive slope, at location y that is within the outer and inner fence, usually indicates that it is a harder sub-layer than its adjacent sublayers.

(2) If there is more than one interface detected within 4 inches (counting from the bottom to the top), the interface around that area can be determined by averaging the locations of the interfaces (Holes 2, 3, 4, 5, 6, 7, and 8 drilled on Block 6), but this averaged location can not compare with the next interface location.

(3) Ignore the interfaces and discontinuities derived from the first 5 inches (from the roof line) and the last 2 inches (hole end). The goal is to avoid the influence from the adjustment of the hydraulic system during startup and finish.

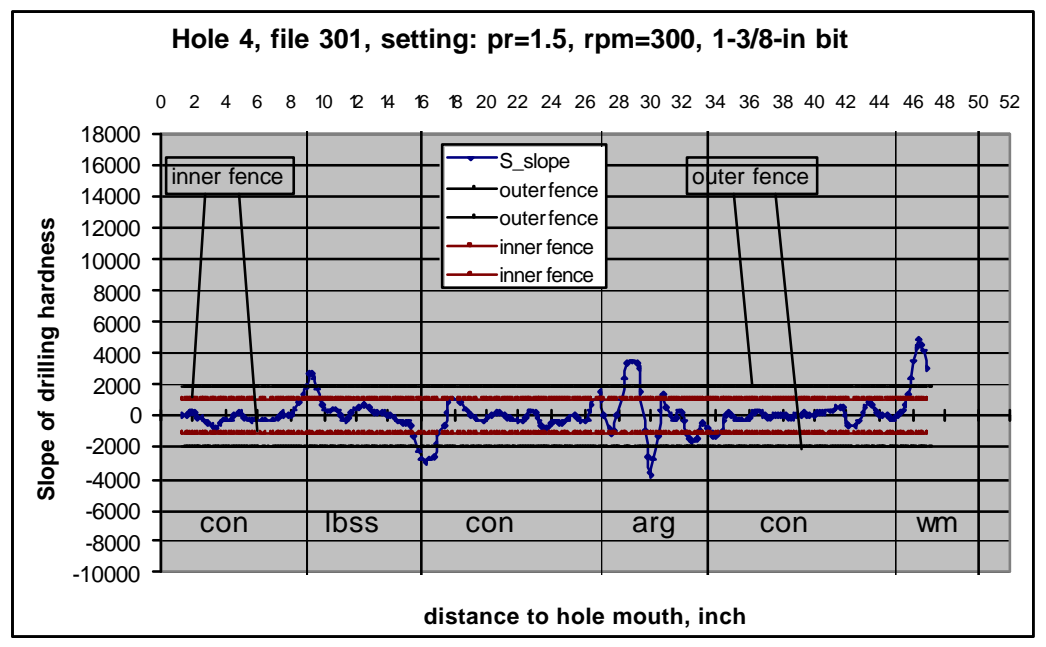

Figure 33 An example of slope distribution along a drilled hole (Hole 4, Block 6)

Figure 35 shows an example of the calculated slope along a drilled hole. The actual locations of the interfaces are 9, 16, 27, 33.5, 45 inches. These outliers shown in Figure 35 indicate the locations of the interfaces between rock layers. It can be seen that the locations of the interfaces derived from the slope of the drilling hardness fr Hole 4 (Figure 35) are 9.11, 16.28, 28.86, 30.09, and 46.39 inches. According to criteria in step 


\section{Development of Algorithms for Determination of Rock Properties}

2 , if there is more than one interface detected within 4 inches (counting from the bottom to the top), the interface around that area can be determined by averaging the locations of the interfaces. Thus interfaces located at 28.86 and 30.09 inches should be averaged. This is how the interface located at 29.475 inches obtained for Hole 4 (Table 14). Since the hole length of Hole 4 is 47.0 inches, the interface, located at 46.39 inches and derived from the drilling hardness should be ignored by applying the criteria in Step 3. It can be seen that around the interfaces between rock layers, the absolute values of slopes were much higher than those within the rock layers. The outliers between inner fence and outer fence are supposed to be indicators of rock discontinuities, which will be verified by the field data later. In this laboratory test, all the detected discontinuities are derived from light brown sandstone, argillite, and white marble rather than concrete. The reason is that the man-made concrete is more uniform in physical property than other rocks.

Table 14 Predicted and actual locations of interfaces on Block 6

\begin{tabular}{|c|c|c|c|c|c|c|}
\hline Hole No. & Location (inch) & if 1 & if 2 & if3 & if4 & if5 \\
\hline \multirow{3}{*}{1} & Actual & 9 & 16 & 27 & 33.5 & 45 \\
\hline & Predicted & N/D & 16.55 & $\mathrm{~N} / \mathrm{D}^{*}$ & 31.52 & 45.82 \\
\hline & Difference & N/D & 0.55 & N/D & 1.98 & 0.82 \\
\hline \multirow{3}{*}{2} & Actual & 9 & 16 & 27 & 33.5 & 45 \\
\hline & Predicted & N/D & $\mathrm{N} / \mathrm{D}$ & 29.51 & 33.88 & 47.57 \\
\hline & Difference & N/D & N/D & 2.51 & 0.38 & 2.57 \\
\hline \multirow{3}{*}{3} & Actual & 9 & 16 & 27 & 33.5 & 45 \\
\hline & Predicted & N/D & 15.47 & 28.355 & 32.29 & 46.03 \\
\hline & Difference & N/D & $\mathbf{0 . 5 3}$ & 1.355 & 1.21 & 1.03 \\
\hline \multirow{3}{*}{4} & Actual & 9 & 16 & 27 & 33.5 & 45 \\
\hline & Predicted & 9.11 & 16.28 & 29.475 & $\mathrm{~N} / \mathrm{D}$ & $\mathrm{N} / \mathrm{D}$ \\
\hline & Difference & 0.11 & 0.28 & 2.475 & N/D & N/D \\
\hline \multirow{3}{*}{5} & Actual & 9 & 16 & 27 & 33.5 & 45 \\
\hline & Predicted & 9.32 & 16.94 & 27.32 & 33.56 & 46.46 \\
\hline & Difference & 0.32 & 0.94 & 0.32 & 0.06 & 1.46 \\
\hline \multirow{3}{*}{6} & Actual & 9 & 16 & 27 & 33.5 & 45 \\
\hline & Predicted & N/D & 15.92 & 28.55 & 34.28 & 46.26 \\
\hline & Difference & N/D & 0.08 & 1.55 & 0.78 & 1.26 \\
\hline \multirow{3}{*}{7} & Actual & 9 & 16 & 27 & 33.5 & 45 \\
\hline & Predicted & $\mathrm{N} / \mathrm{D}$ & 17.32 & 27.67 & 34.38 & 47.2 \\
\hline & Difference & N/D & 1.32 & 0.67 & 0.88 & 2.2 \\
\hline
\end{tabular}




\section{Development of Algorithms for Determination of Rock Properties}

\begin{tabular}{|c|c|c|c|c|c|c|}
\hline \multirow{3}{*}{8} & Actual & 9 & 16 & 27 & 33.5 & 45 \\
\cline { 2 - 7 } & Predicted & N/D & 15.77 & 28.675 & 33.68 & 46.11 \\
\cline { 2 - 7 } & Difference & N/D & $\mathbf{0 . 2 3}$ & $\mathbf{1 . 6 7 5}$ & $\mathbf{0 . 1 8}$ & $\mathbf{1 . 1 1}$ \\
\hline
\end{tabular}

*N/D - not available

Table 14 and Figures 34 to 40 show the analysis results from the holes drilled in Block 6 , and the predicted and actual locations of the interfaces between rock layers are also presented. For this block, only 6 rock layers were drilled through from the bottom to the top. Thus there are 5 interfaces between the 6 layers, which are called in Table 14 if 1 (con-lbss), if2 (lbss-con), if3 (con-arg), if4(arg-con), if5 (con-wm). Here, con means concrete, lbss means light brown sandstone, arg means argillite, and wm means white marble. For the eight drilled holes, there are $40(8 \times 5)$ interfaces drilled through and 30 of them, up to $75 \%$, , are detectable. Most of the not-detectable interfaces are if 1 and if 2 . The maximum, minimum and average errors between the predicted and actual locations of detected interfaces are 2.57, 0.06 and 1.028 inches, respectively. Thirty predicted interfaces up to $75.0 \%$ have errors less than 3.0 inches.

Figures 34, 35, 36, 37, 38, 39 and 40 and Table 14 also show the prediction results from holes 1, 2, 3, 5, 6, 7, and 8. The variations in the drilling hardness in holes 1 and 2 (Figures 34 and 35) are not as clear as other 6 holes. The major reason may be the lower thrust cap and the controlled penetration rate and rotation rate set before drilling these two holes (see Table 13). From the distribution of the drilling hardness in each rock layer, it seems that the drilling hardness calculated from holes 5, 6, 7 and 8 are more consistent than holes 1 and 2 except for argillite. The distribution of drilling hardness and slope for Holes 3, 5, 6, 7, and 8 indicate that there are some interfaces detected by using the criteria (Step 1) within argillite. If looking at the distribution of the drilling hardness in argillite, it can be seen that the drilling hardness at the location where the slope shows it is an interface is either higher or lower than its adjacent rock. These either higher or lower drilling hardness, on the one hand, shows the complexity of rock properties that vary a lot even within one rock layer; On the other hand, these ever-changing rock properties complicate the identification. Another reason for the extra interfaces or discontinuities detected from the argillite is that while drilling in the argillite, the whole block started to 


\section{Development of Algorithms for Determination of Rock Properties}

move back and forth because argillite is the hardest rock layer on this block. These activities from the block would cause vibration on the block and drilling, thus yielded some noise data. However, the extra interfaces detected near the end of the hole are caused by the rock properties and the adjustment of the hydraulic system. The reason is that the data monitoring system is still working before the machine totally stops. In order to eliminate these noise points, the interfaces and discontinuities derived the last two inches should be ignored.

Since light brown sandstone, argillite, and white marble are stronger than concrete and concrete was embedded between the above rock layers, the transitions for the 40 interfaces can be divided into two types, drilling from hard to weak and drilling from weak to hard. Table 14 shows that, when drilling from soft to hard, there are 16 out of 24 interfaces were detected, up to $66.67 \%$; when drilling from hard to weak, there are 14 out of 16 interfaces were detected, up to $87.5 \%$.

It can be inferred from the above analysis and results that the variation in the drilling hardness can be used to indicate the presence of interfaces between rock layers, change of lithology, and even the competency of the rock.

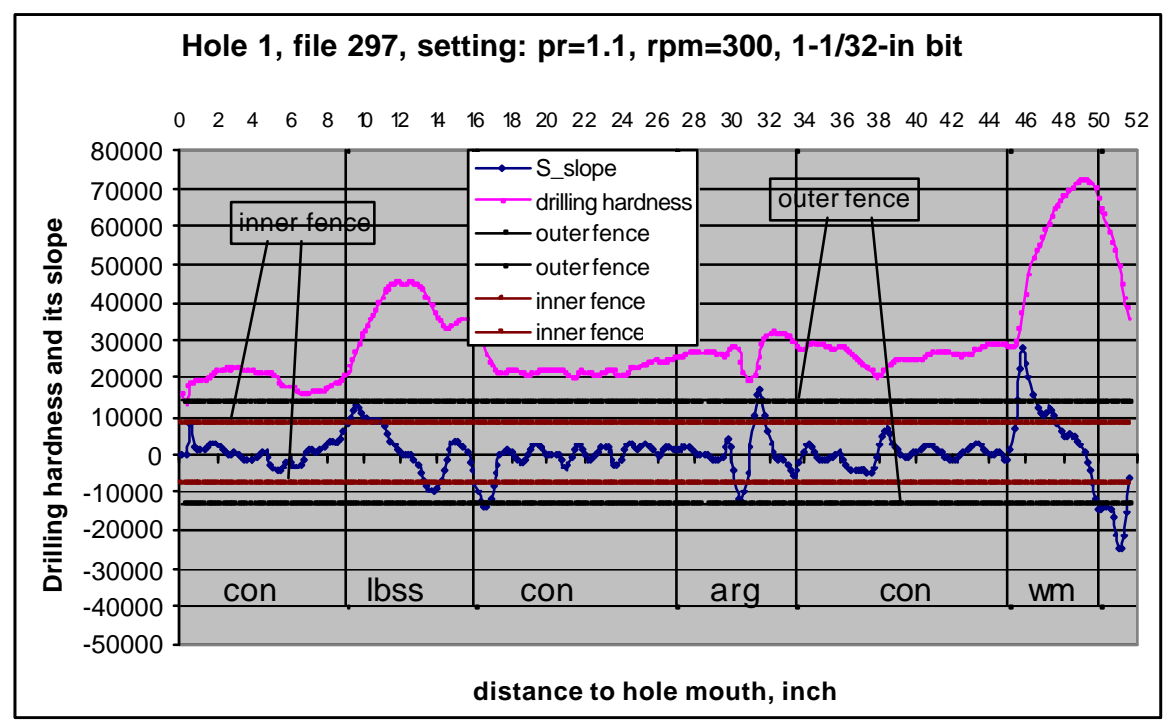

Figure 34 Drilling hardness and its slope distribution along a drill hole (Hole 1, Block 6) 


\section{Development of Algorithms for Determination of Rock Properties}

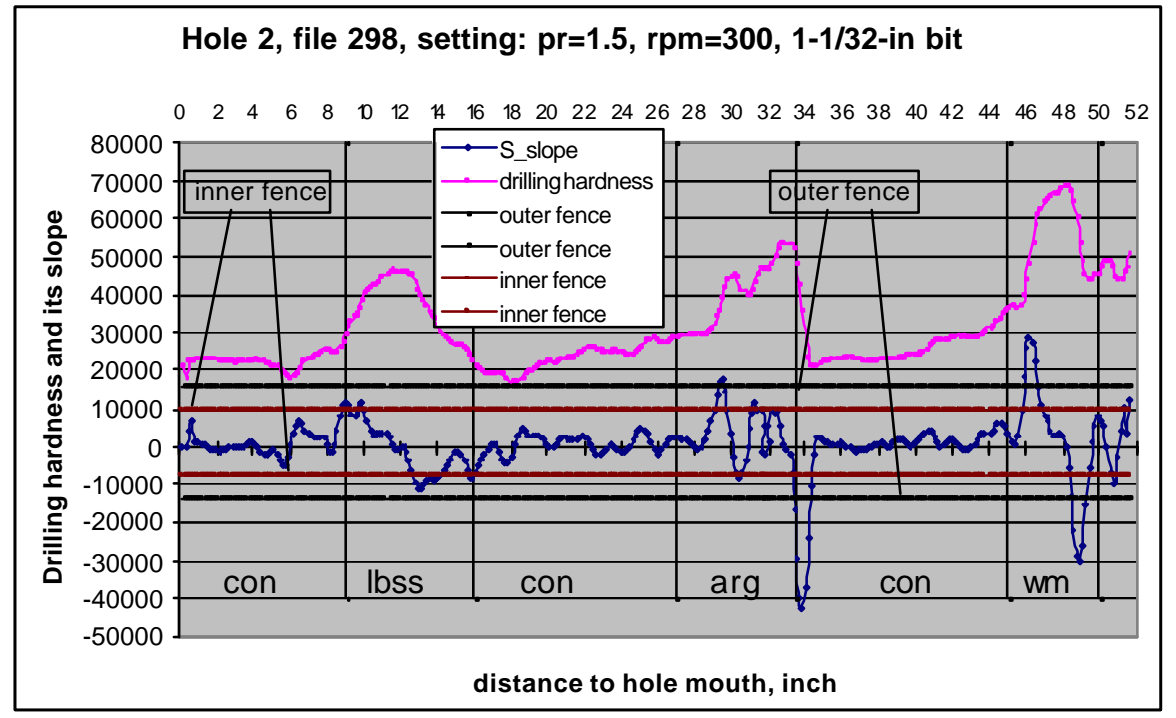

Figure 35 Drilling hardness and its slope distribution along a drill hole (Hole 2, Block 6)

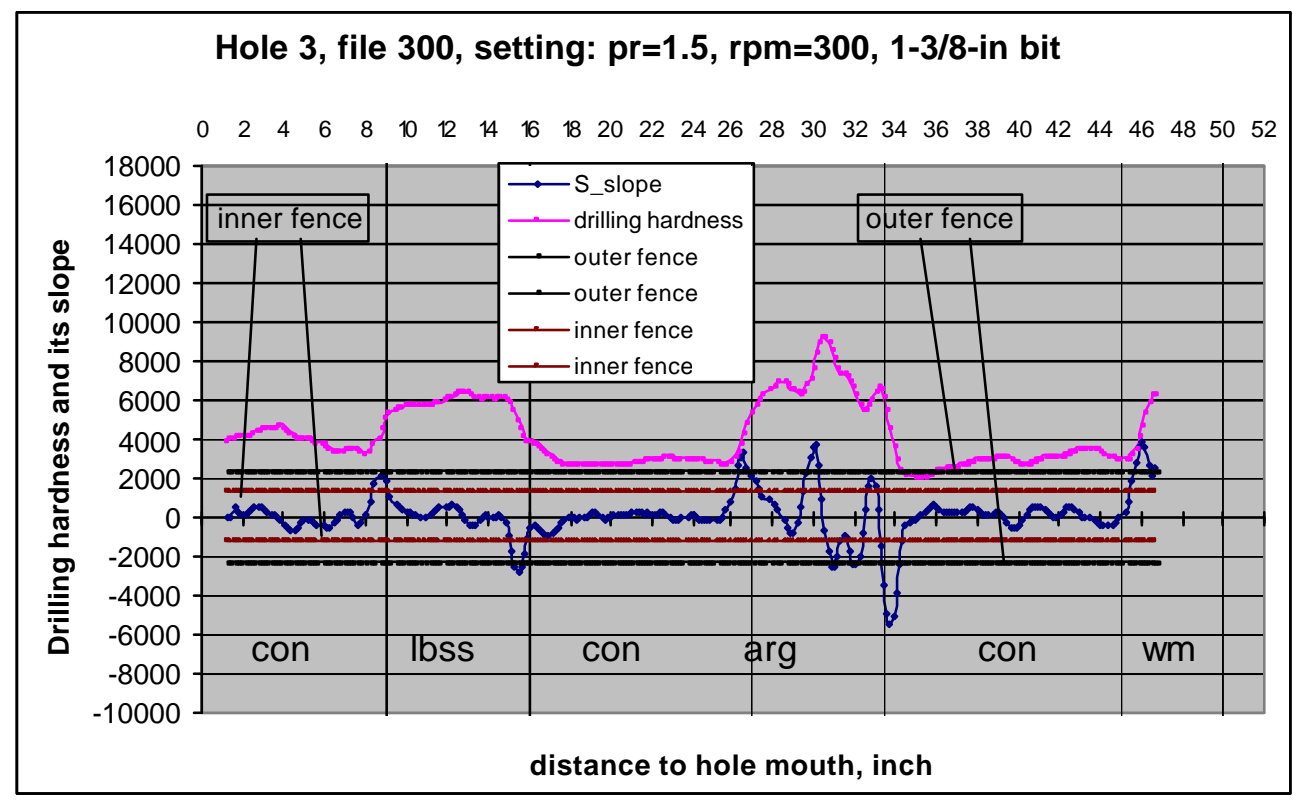

Figure 36 Drilling hardness and its slope distribution along a drill hole (Hole 3, Block 6) 


\section{Development of Algorithms for Determination of Rock Properties}

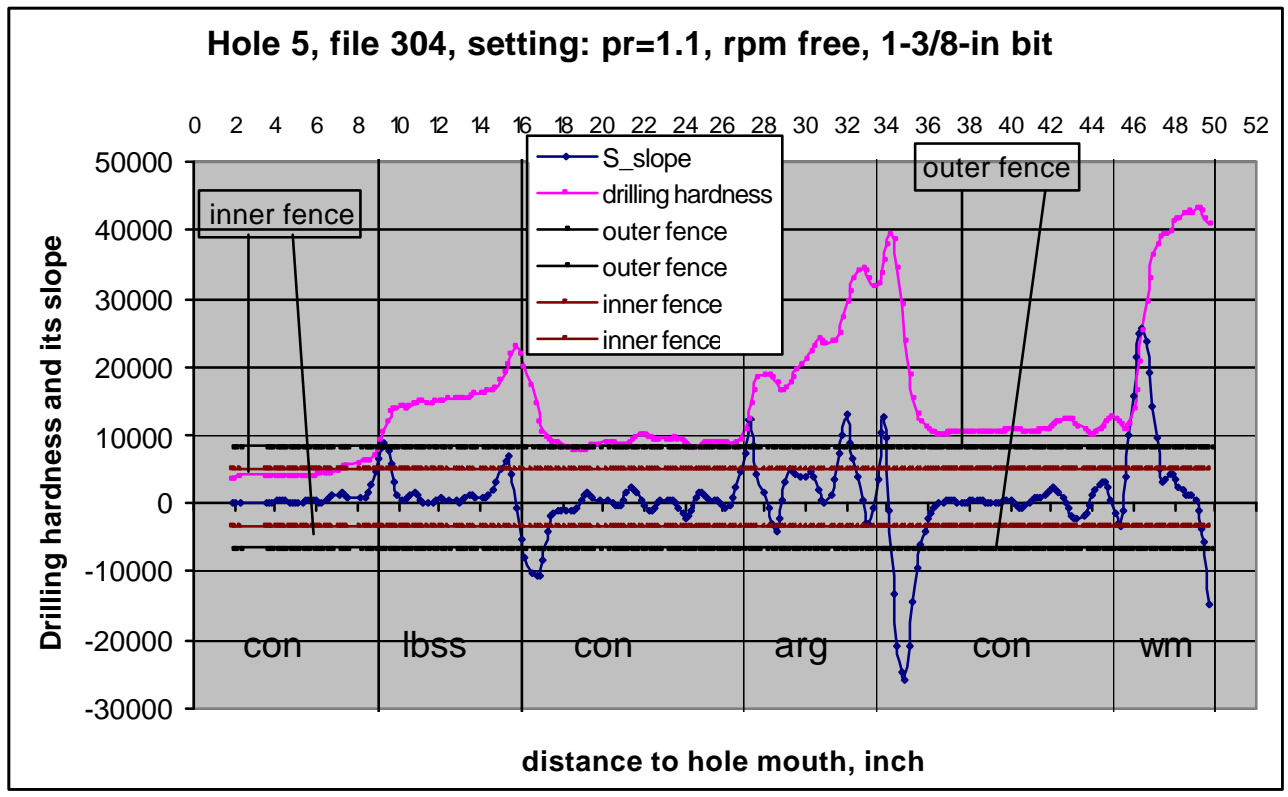

Figure 37 Drilling hardness and its slope distribution along a drill hole (Hole 5, Block 6)

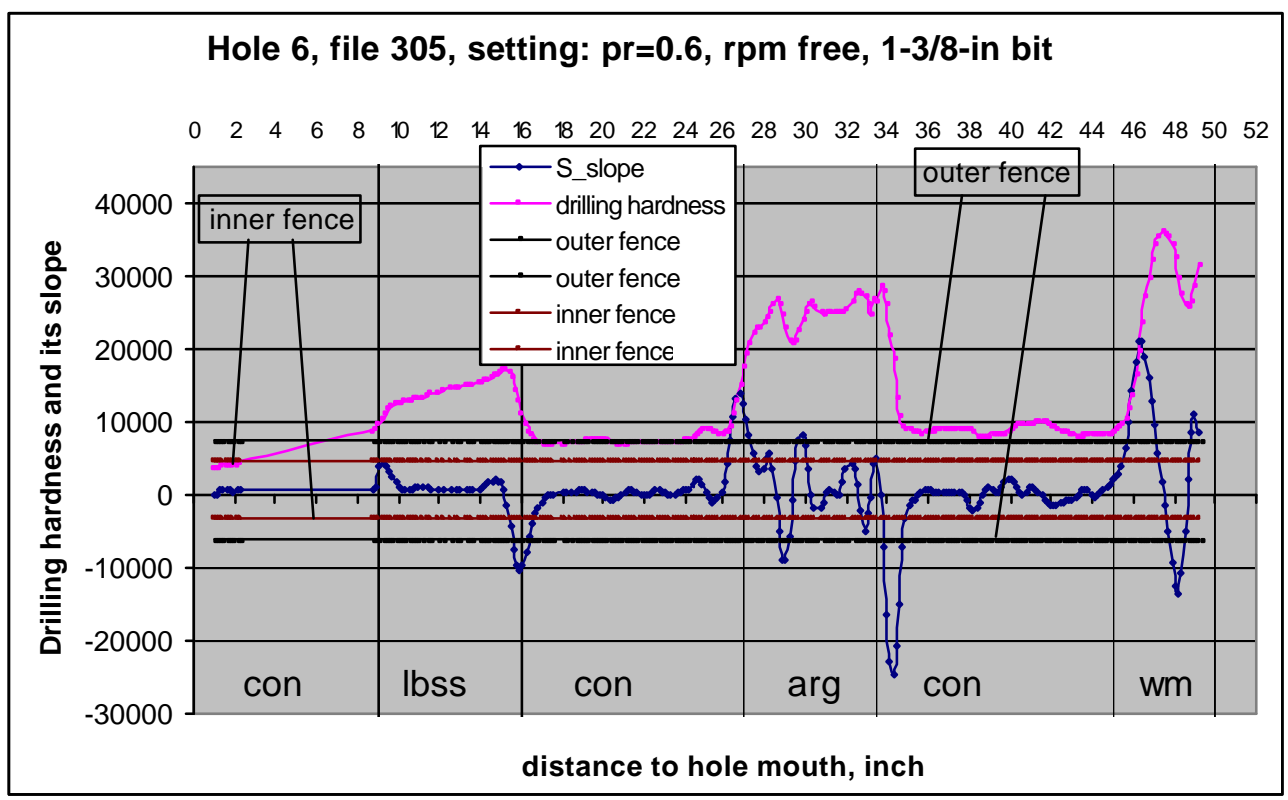

Figure 38 Drilling hardness and its slope distribution along a drill hole (Hole 6, Block 6) 


\section{Development of Algorithms for Determination of Rock Properties}

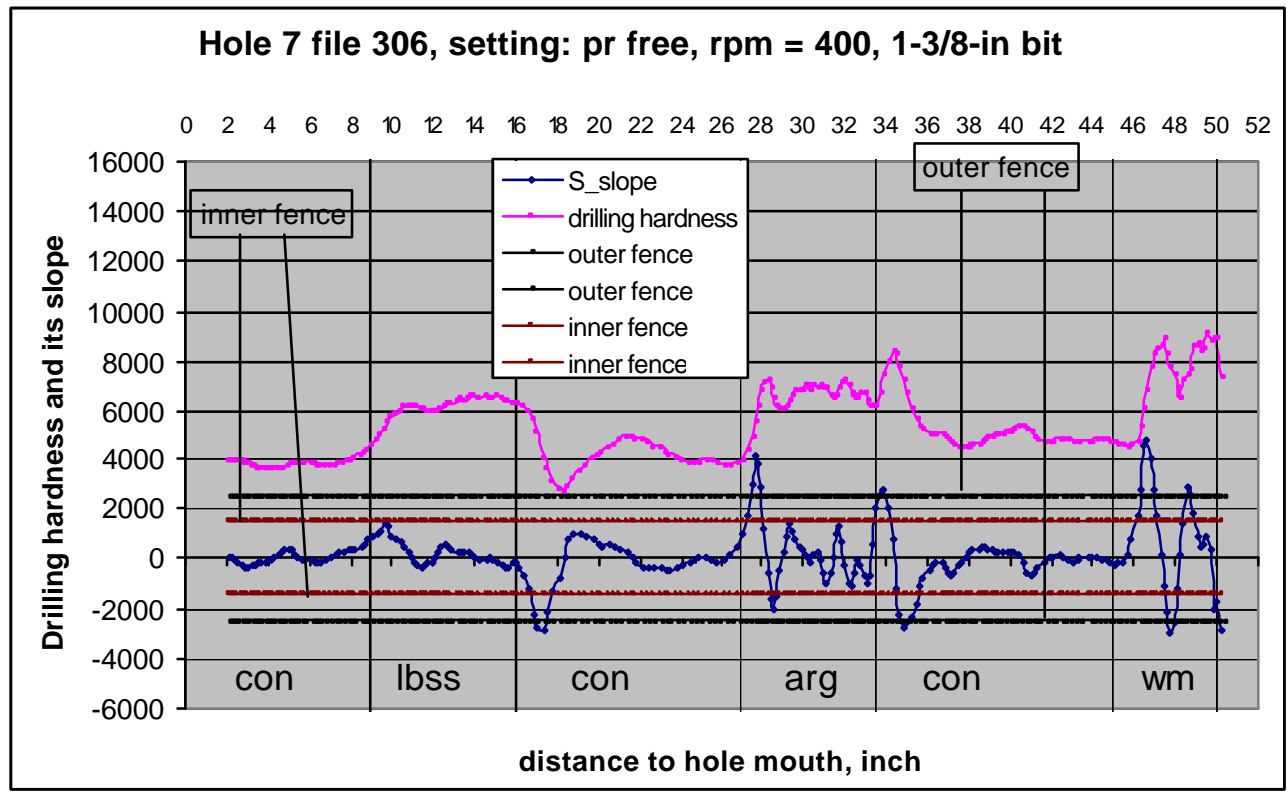

Figure 39 Drilling hardness and its slope distribution along a drill hole (Hole 7, Block 6)

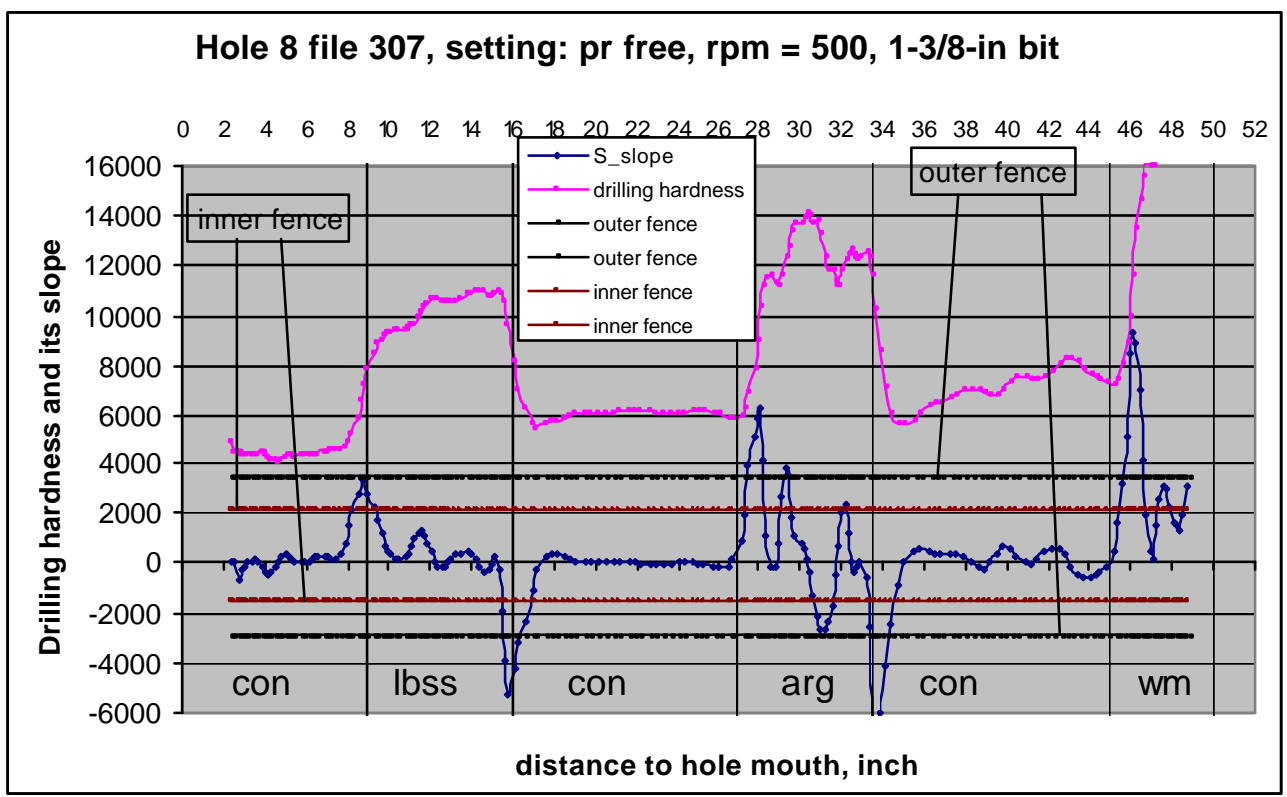

Figure 40 Drilling hardness and its slope distribution along a drill hole (Hole 8, Block 6) 


\section{Development of Algorithms for Determination of Rock Properties}

\subsection{The Energy/Work Lost during Drilling Process}

As previously depicted, drilling is a process that transfers mechanical energy (work) to the rock in the form of thrust and/or torque to overcome resistance from the drilled rock. During the drilling process, some of the energy is spent on moving roof bolter drill head up with a certain speed (in the form of kinetic energy and potential energy), some of the energy is expended on rod-to-rock transition in the form of torsion energy, and some of the energy is spent on the friction between the rock and the drill bit. In this section, these energy losses are analyzed.

\subsubsection{Energy Lost in Rotational Direction}

In the rotational direction, the work done by torque from point $i$ to $i+1$ is mainly composed of three parts (Eq.20), kinetic energy, torsion energy and thermal energy caused by the friction between the drill bit and rock in the rotational direction. Among the three parts, the heat energy contributes to rock grinding and breaking the rock out, while the kinetic and torsion energies are spent on the drill rod and bit.

Figure 41 shows how the total work done by torque, torsion energy and kinetic energy varied along a drilled hole (Hole 4, Block 6). Torsion energy is proportional to the torque and length of the drill rod (see Eq.20): the higher torque and the longer the drill rod are, the higher the torsion energy is. In this drilling case, the length of the rod did not change. Therefore, the variation in the torsion energy depended solely on changes in torque. Comparatively, the kinetic energy is the smallest one among the three kinds of energies in the rotational direction, and the torsion energy is larger than the kinetic energy but it only accounts for a very small portion of the total work done by the torque. This result also shows that most of the work done by torque is consumed in breaking and overcoming the frictional resistance of rock.

By combining the total data sets (2212) obtained from the eight holes drilled in the Block 6, the percentages of torsion energy and kinetic energy in the drilling and rotational directions were calculated. The histograms in Figures 42 and 43 show that, in 


\section{Development of Algorithms for Determination of Rock Properties}

most cases, the energy expended on torsion ranges from $2 \%$ to $8 \%$, and on kinetic energy, 0 to $0.8 \%$. These results show that in the rotational direction, at least $92 \%$ of the work done by toque is consumed in breaking and overcoming the frictional resistance of rock in the form of grinding and removing rock fragments and producing a certain amount of heat.

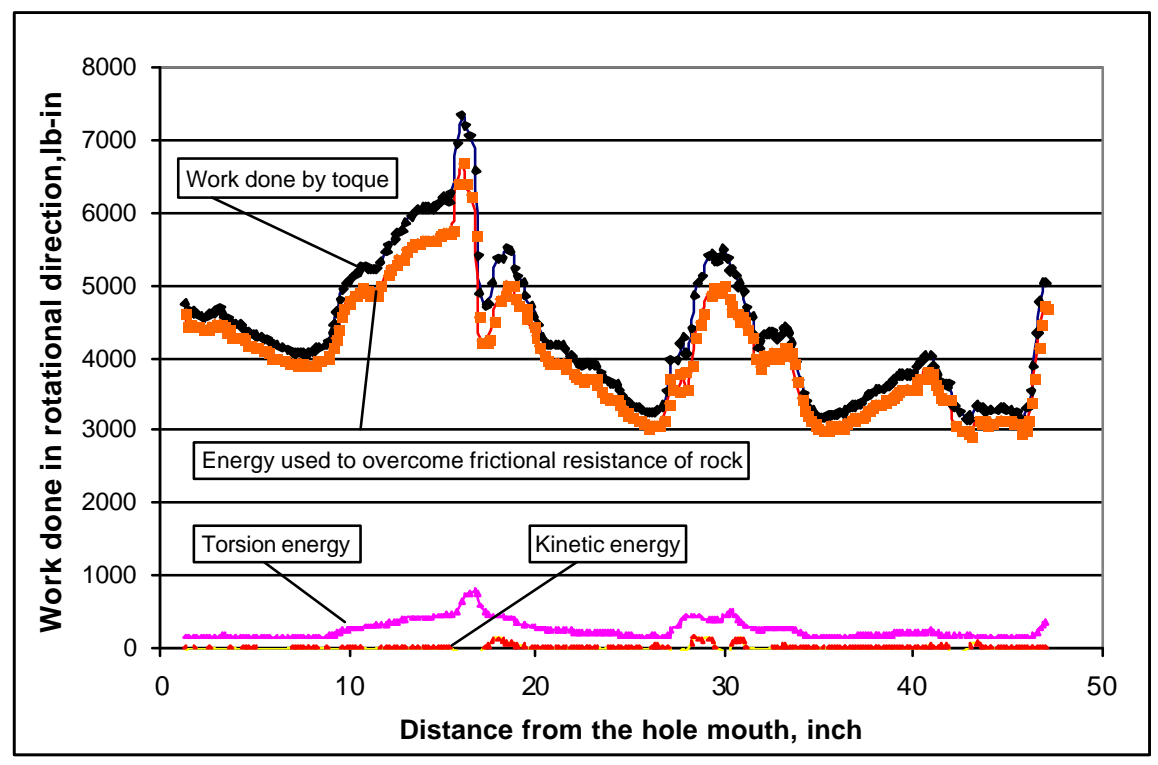

Figure 41 An example of energy variation in the rotational direction (Hole 4, Blcok 6)

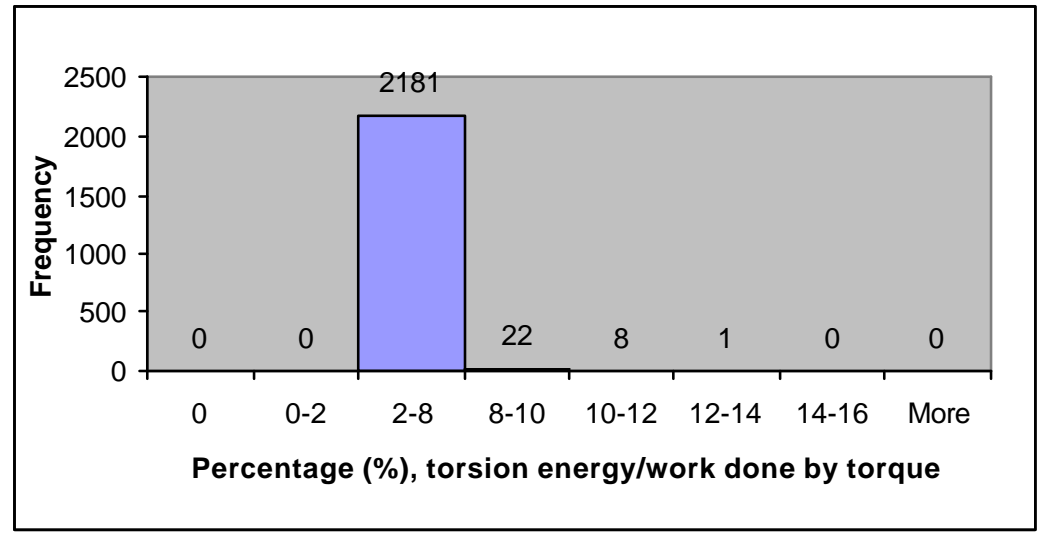

Figure 42 Percentage of torsion energy in the work done by torque for all 8 holes in Block 6 


\section{Development of Algorithms for Determination of Rock Properties}

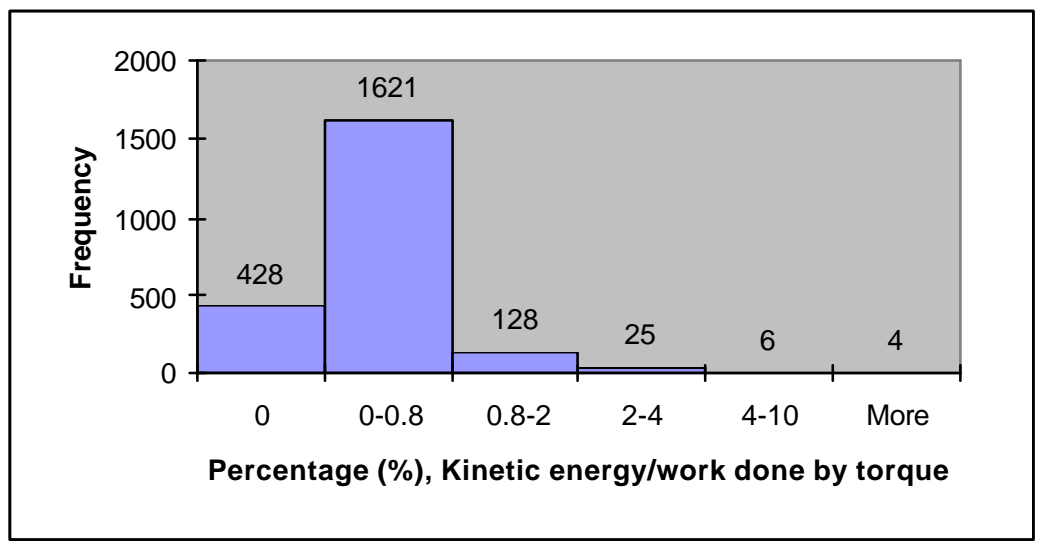

Figure 43 Percentage of kinetic energy in the work done by torque for all 8 holes in Block 6

\subsubsection{Energy Lost in the Drilling Direction}

In the drilling direction, the work done by thrust from point $\mathrm{i}$ to $\mathrm{i}+1$ is mainly composed of four parts (see Eq.23), kinetic energy, potential energy, work to overcome the friction between the drill bit and rock in the drilling direction, and the energy consumed in breaking the cementing bond of or penetrating into the drilled rock. Among the four parts, only the energy consumed in breaking the cementing bond of or penetrating into the rock is directly contributed to the rock breakage, and the remaining parts are lost in the form of kinetic, potential and thermal energies.

Figure 44 shows an example of how kinetic energy, potential energy and work done to overcome the frictional resistance of rock in the drilling direction. It can be seen that kinetic energy, potential energy and the work done to overcome the friction varied a little bit while drilling in different positions in the rock layers, and depended on how strong the rock layers were. In other words, a small portion of the work done by thrust is contributed to kinetic energy, potential energy and overcoming the frictional resistance of rock in the drilling direction, while a great majority of it is contributed to the rock breakage. 


\section{Development of Algorithms for Determination of Rock Properties}

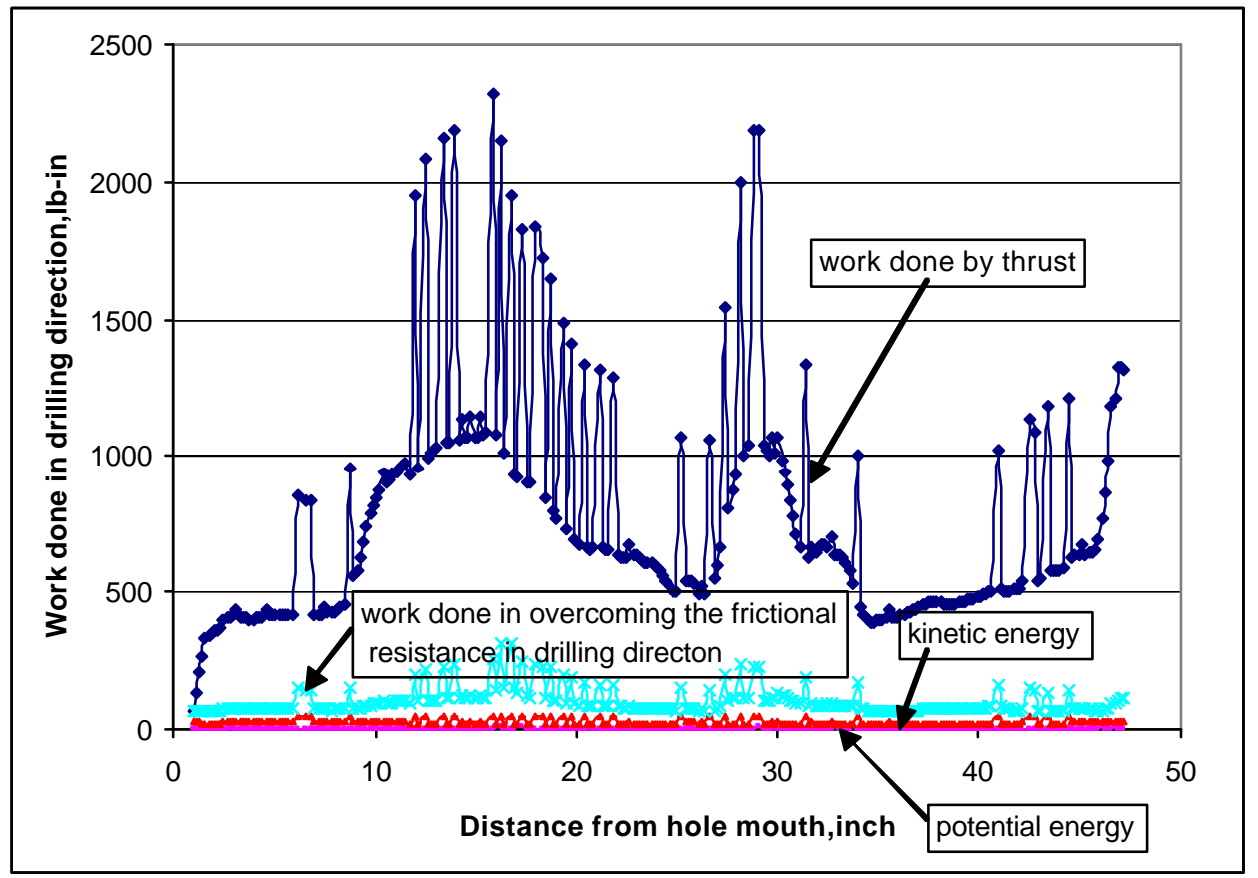

Figure 44 Work done in drilling direction (Hole 4, Block 6)

The histograms in Figures 45, 46, 47 and 48 show the frequency with which each kind of energy loss was within a certain percentage band of the entire energy from all 8 holes drilled in the Block 6, the layered block. The percentage of the total work lost ranges from 10 to $30 \%$ (Figure 45), the percentage of the kinetic energy lost is about $1 \%$ (Figure 46), the potential energy lost ranges from 2 to $8 \%$ (Figure 47), and the work done to overcome frictional resistance of rock in the drilling direction varies from 10 to $20 \%$ (Figure 48). Most of the energy lost in the drilling direction is the results of the friction between the drill bit and the drilled rock. 


\section{Development of Algorithms for Determination of Rock Properties}

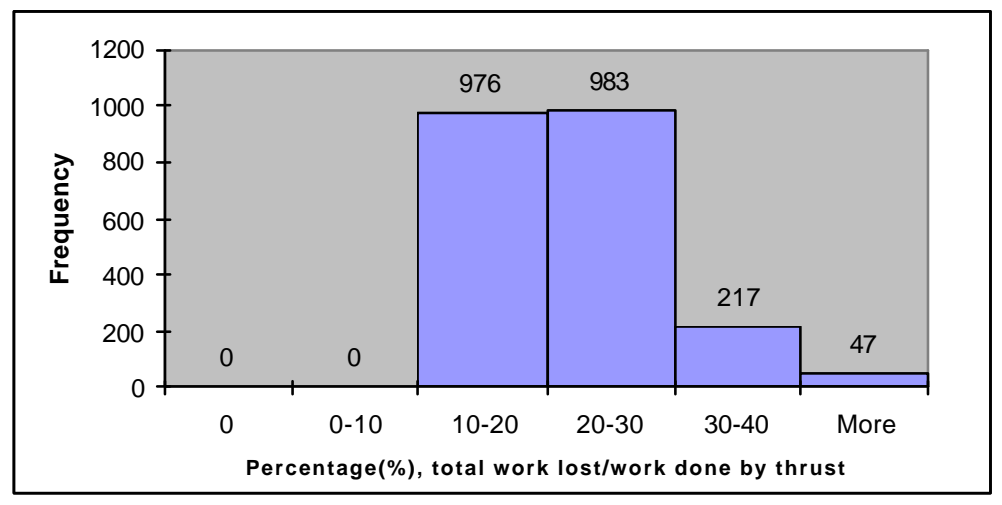

Figure 45 Percentage of the total energy lost in the work done by thrust for all 8 holes in Block 6

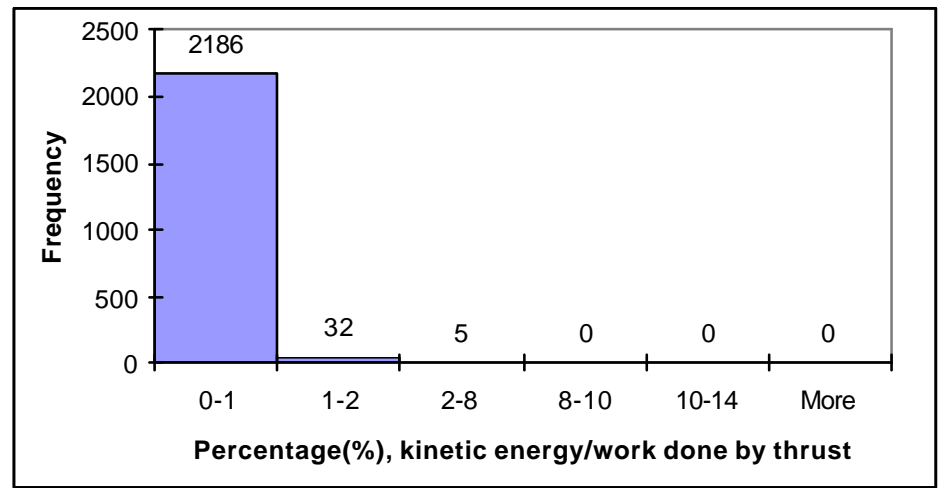

Figure 46 Percentage of kinetic energy in the work done by thrust for all 8 holes in Block 6

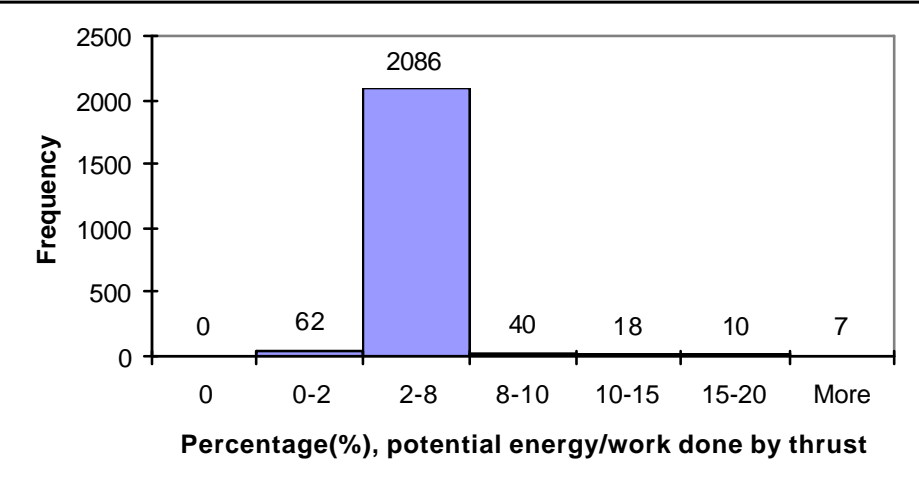

Figure 47 Percentage of potential energy in the work done by thrust for all 8 holes in Block 6 


\section{Development of Algorithms for Determination of Rock Properties}

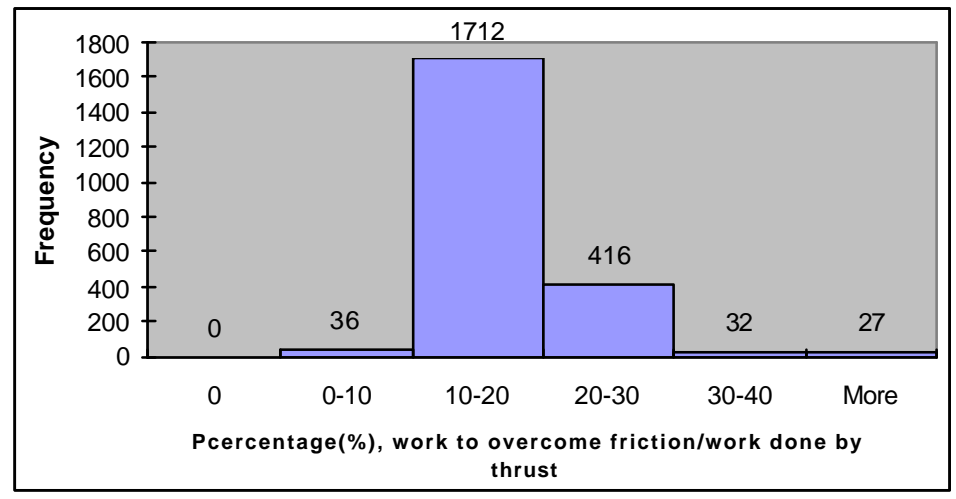

Figure 48 Percentage of work done to overcome friction in the work done by thrust for all 8 holes in Block 6

The analysis of energy lost in the drilling direction indicates that in Eq. 23 the thrust, which directly contributes about $70 \%$ energy to the rock breakage, plays a vital role in calculating the drilling hardness. Since the thrust is a controllable parameter, it needs to pre-set an upper limit as high as possible in order to ensure the drill bit will penetrate into all rock strata in the entry roof. If the pre-set thrust cap is not high enough, it can not ensure the drill bit will penetrate into the rock and the achievement of the pre-set penetration rate, thus significantly influence the drilling process and the derived parameter, drilling hardness.

By considering the energy lost in both the rotational and drilling directions, the total energy lost is around 20 to $30 \%$ of the total mechanical energy done by the thrust and torque. In other words, about $70 \%$ of the work done by thrust and $92 \%$ of the work done by torque are directly contributed to the rock breakage. In the rotational direction, 2 to $8 \%$ is lost in the torsion of the drill rod, and 0 to $0.8 \%$ is lost in the kinetic energy. In the drilling direction, about $1 \%$ is lost in kinetic energy, 2 to $8 \%$ is lost in potential energy, and 10 to $20 \%$ is lost in overcoming the friction between the drill bit and the drilled rock.

From the analysis above, it can be concluded that energy lost during drilling process is an indispensable factor and can not be ignored. Those lost energy show that any of the 


\section{Development of Algorithms for Determination of Rock Properties}

original drilling parameters, such as thrust, torque, rotation rate, and penetration rate, can not be relied on solely as the indicator to detect the locations of discontinuities. The derived parameter used as an indicator of the locations of the discontinuities must be as far as possible to take the energy lost into account, and that is what the new drilling parameter, drilling hardness does. However, the drilling hardness still composes of noise portion caused by the (delayed) adjustment time of the hydraulic system and this research does not take it into account.

In addition, the variable energies lost along a hole also show that the lost energy is not a constant even when drilling in the same rock, which is caused by the variable properties due to the granular structure of rock. For instance, the torsion energy consists of the torque component, when drilling in different rock layers, the required torque will change correspondingly. Finally, the varied torque will make the torsion energy change, which does not contribute to rock breakage. In other words, the varied rock properties can indirectly affect the values of the lost energy, such as torsion energy.

\subsection{The Choice of Controlling Parameters}

The laboratory test conducted on Blocks 2, 3, and 4 (Figures 16 and 17) did not consider the factor of thrust cap. Those tests were conducted with penetration rate and rotation rate controlled. For all other tests, thrust cap was considered. In conjunction with the thrust cap, three control modes are available: (1) Penetration rate is controlled and rotation rate is free to run; (2) rotation rate is controlled and penetration rate is free to run; and (3) Penetration rate and rotation rate are controlled simultaneously.

Actually, the variations of the recorded drilling parameters including the derived drilling parameter, drilling hardness, are inevitably affected by the rock properties, energy lost, and the adjustment of the hydraulic system. In the drilling mechanical model (DMM) defined in Section 4.1, the variations in rock properties can be identified by the drilling hardness which considers the lost energies including kinetic energy, potential energy, torsion energy, and the heat energy caused by the friction between the drill bit and rock. Although the drilling hardness, to a certain degree, reduces parts of the noise 


\section{Development of Algorithms for Determination of Rock Properties}

from the energy lost, the remaining noise, particularly that from near the interfaces between rock layers caused by the adjustment of the hydraulic system remains.

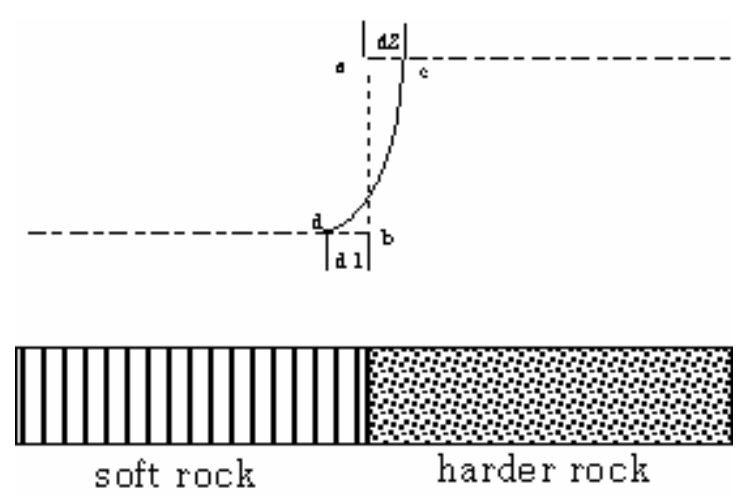

Figure 49 Ideal transition of a drilling parameter near the interface between rock layers

The dash lines in Figure 49 show an ideal variation of a drilling parameter while drilling between two different rock layers. Under this ideal condition, the recorded drilling parameter maintains a constant within one rock layer and jumps from point $b$ right at the edge of the soft rock to point a, which is also right at the edge of the harder rock. Unfortunately, the recorded drilling parameter does not go this way in the laboratory or field tests because of the ever-changing rock properties and the corresponding adjustment of the hydraulic system. Usually, the recorded values of the drilling parameter varies by following $d \rightarrow c$ rather than $b \rightarrow a$. The variation of the drilling parameter (e.g. drilling hardness) near the interface does not start and end right at points $\mathrm{b}$ and $\mathrm{a}$ but $\mathrm{d}$ and $\mathrm{c}$ respectively. When drilling near the interface, the rock properties start to change. This will cause changes in penetration rate or rotation rate and the hydraulic system will also adjust correspondingly in order to maintain the pre-set penetration rate or rotation rate. The response time or the adjustment time of the hydraulic system is in the range of 0.3 seconds ${ }^{[23]}$. In addition, the adjustment of the flow rate is gradual rather than sudden jump from low flow to high flow and vice versa. Therefore, the variations of rock properties and the gradual adjustment of the hydraulic system determine that the variation of the drilling hardness (or thrust) go along $d \rightarrow c$ rather than $b \rightarrow a$. The length of $\mathrm{d} 1$ and $\mathrm{d} 2$ partly depends on the adjustment time of the hydraulic system. The shorter the 


\section{Development of Algorithms for Determination of Rock Properties}

adjustment time are, the shorter the $\mathrm{d} 1$ and $\mathrm{d} 2$ are. If the adjustment time is shorter, it means the hydraulic flow can change more quickly to increase or reduce thrust and torque when drilling from one rock layer to another, thus the transition zone of thrust and torque can be shorten.

In an attempt to determine which control mode will yield a better result, the DMM and drilling hardness developed in Sections 4.1 and 4.2 were applied to the test results obtained from all manufactured blocks; i.e. Blocks 2, 3, and 4 in Figures 16 and 17, the Fractured Block in Figure 50, the Solid Block in Figure 56, and Block 6 in Figure 27. All the processed results in drilling hardness and slope are shown in Appendix II.

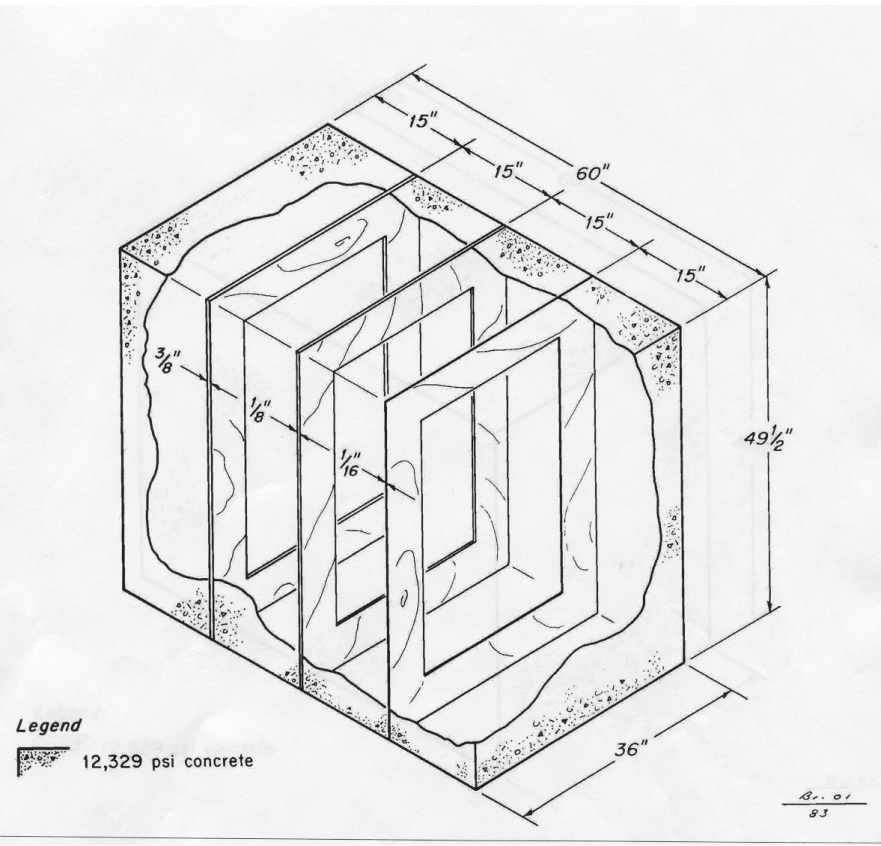

Figure 50 Fractured Block 


\section{Development of Algorithms for Determination of Rock Properties}

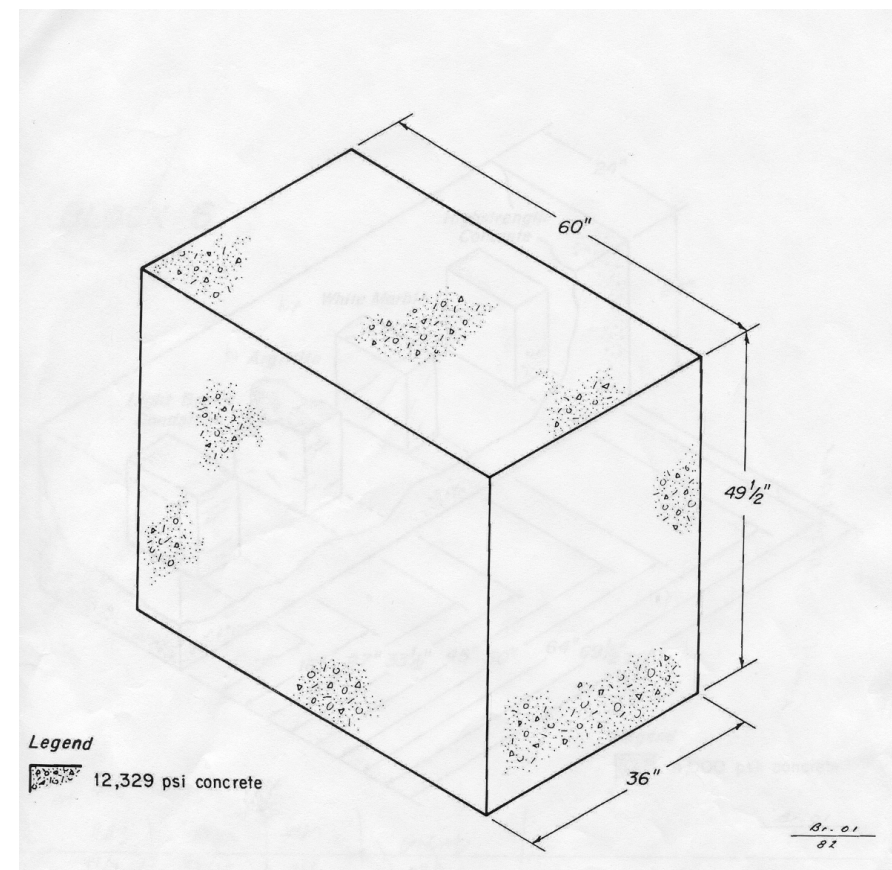

Figure 51 Solid Block

The control parameters of holes drilled in the Fractured Block, Solid Block, and Block 6 are shown in Tables 15, 16, and 13 respectively.

Table 15 Laboratory testing control parameters for Fractured Block

\begin{tabular}{|c|c|c|c|c|c|}
\hline Hole No. & $\begin{array}{c}\text { Bit size } \\
\text { inch }\end{array}$ & $\begin{array}{c}\text { P.R } \\
\text { in/sec }\end{array}$ & $\begin{array}{c}\text { rotation rate } \\
\text { Ro./min. }\end{array}$ & $\begin{array}{c}\text { Thrust cap } \\
\text { psi }\end{array}$ & $\begin{array}{c}\text { Hole Length } \\
\text { in }\end{array}$ \\
\hline 1 & $1-1 / 32$-in & 0.4 & 300 & 848.7 & 52.0 \\
\hline 2 & $1-1 / 32-$ in & 0.8 & 300 & 848.7 & 52.0 \\
\hline 3 & $1-1 / 32-$ in & 1.5 & 300 & 848.7 & 52.0 \\
\hline 4 & $1-1 / 32-$ in & 0.4 & 400 & 848.7 & 52.0 \\
\hline 5 & $1-1 / 32-$ in & 0.8 & 450 & 848.7 & 52.0 \\
\hline 6 & $1-1 / 32-$ in & 1.5 & 500 & 848.7 & 52.0 \\
\hline 7 & $1-1 / 32-$ in & 1.5 & 300 & 848.7 & 52.0 \\
\hline
\end{tabular}




\section{Development of Algorithms for Determination of Rock Properties}

Table 16 Laboratory testing control parameters for Solid Block

\begin{tabular}{|c|c|c|c|c|c|}
\hline Hole No. & $\begin{array}{c}\text { Bit size } \\
\text { inch }\end{array}$ & $\begin{array}{c}\text { P.R } \\
\text { in/sec }\end{array}$ & $\begin{array}{c}\text { rotation rate } \\
\text { Ro./min. }\end{array}$ & $\begin{array}{c}\text { Thrust cap } \\
\text { psi }\end{array}$ & $\begin{array}{c}\text { Hole Length } \\
\text { in }\end{array}$ \\
\hline 28 & $1-3 / 8$-in & 1.1 & 300 & 700 & 50.5 \\
\hline 29 & $1-3 / 8$-in & 1.1 & 500 & 700 & 50.5 \\
\hline 30 & $1-3 / 8$-in & 1.1 & 300 & 900 & 50.5 \\
\hline 31 & $1-3 / 8$-in & 1.1 & 500 & 900 & 50.5 \\
\hline 32 & $1-3 / 8$-in & 1.1 & 300 & 1100 & 50.5 \\
\hline 33 & $1-3 / 8$-in & 1.1 & 500 & 1100 & 51 \\
\hline 34 & $1-3 / 8$-in & 1.1 & 300 & 600 & 52.5 \\
\hline 35 & $1-3 / 8$-in & 1.1 & 500 & 600 & 52.5 \\
\hline 36 & $1-3 / 8$-in & 1.1 & 300 & 700 & 52.0 \\
\hline 37 & $1-3 / 8$-in & 1.1 & 500 & 700 & 52.0 \\
\hline 38 & $1-3 / 8$-in & 1.1 & 300 & 800 & 52.5 \\
\hline 39 & $1-3 / 8$-in & 1.1 & 500 & 800 & 52.5 \\
\hline
\end{tabular}

\subsubsection{Test Results for Blocks 2, 3, and 4}

These three blocks (Figures 16 and 17) were drilled without considering the thrust cap. The control mode was constant penetration rate and rotation rate simultaneously. The analysis in this section mainly focuses on the comparison between the predicted and actual interfaces. All the discontinuities detected within different rock layers will be ignored.

The processed results in the drilling hardness and its slope obtained from Blocks 2, 3 and 4 are shown in Figures II-1 to 27, Appendix II. Tables 17, 18, and 19 show the derived locations of the interfaces between rock layers.

For Block 2, 9 rock layers were drilled through from the bottom to the top, thus there are 8 interfaces between the 9 layers, which are called in Table 17 if1 (con-bss), if 2 (bss-con), if3 (con-lbss), if4 (lbss-con), if5 (con-bss), if6 (bss-con), if7 (con-rss) and if8 (rss-con). Here, con means concrete, bss means brown sandstone, lbss means light brown sandstone, and rss means red sandstone. 


\section{Development of Algorithms for Determination of Rock Properties}

For Block 3, 9 rock layers were drilled through from the bottom to the top, thus there are 8 interfaces between the 9 layers, which are called in Table 18 if1 (con-lbss), if2 (lbss-con), if3 (con-Hcon), if4 (Hcon-con), if5 (con-wm), if6 (wm-con), if7 (con-rss) and if8 (rss-con). Here, con means concrete, Hcon means high strength concrete, lbss means light brown sandstone, wm means while marble, and rss means red sandstone.

For Block 4, 9 rock layers were drilled through from the bottom to the top, thus there are 8 interfaces between the 9 layers, which are called in Table 19 if1 (con-rss), if2 (rsscon), if3 (con-Arg), if4 (Arg-con), if5 (con-wm), if6 (wm-con), if7 (con-Hcon) and if8 (Hcon-con). Here, con means concrete, Hcon means high strength concrete, Arg means Argillite, wm means whilte marble, and rss means red sandstone.

Table 17 Predicted and actual locations of interfaces on Block 2

\begin{tabular}{|c|c|c|c|c|c|c|c|c|c|}
\hline Hole No. & Location (inch) & if1 & if 2 & if3 & if4 & if5 & if6 & if7 & if8 \\
\hline \multirow{3}{*}{14} & Actual & 6.75 & 18.25 & 24.0 & 34.75 & 42 & 54 & 64 & 70.75 \\
\hline & Predicted & N/D & 15.61 & N/D* & $\mathrm{N} / \mathrm{D}$ & $\mathrm{N} / \mathrm{D}$ & N/D & 60.2 & 70.59 \\
\hline & Difference & N/D & 2.64 & N/D & N/D & N/D & N/D & 3.8 & 0.16 \\
\hline \multirow{3}{*}{15} & Actual & 6.75 & 18.25 & 24 & 34.75 & 42 & 54 & 64 & 70.75 \\
\hline & Predicted & N/D & 17.76 & N/D & $\mathrm{N} / \mathrm{D}$ & $\mathrm{N} / \mathrm{D}$ & N/D & 61.79 & 71.94 \\
\hline & Difference & N/D & 0.49 & N/D & N/D & N/D & N/D & 0.1 & 1.25 \\
\hline \multirow{3}{*}{16} & Actual & 6.75 & 18.25 & 24 & 34.75 & 42 & 54 & 64 & 70.75 \\
\hline & Predicted & N/D & $\mathrm{N} / \mathrm{D}$ & $\mathrm{N} / \mathrm{D}$ & $\mathrm{N} / \mathrm{D}$ & 44.73 & N/D & N/D & 73.92 \\
\hline & Difference & N/D & N/D & N/D & N/D & 2.38 & N/D & N/D & 3.17 \\
\hline \multirow{3}{*}{17} & Actual & 6.75 & 18.25 & 24 & 34.75 & 42 & 54 & 64 & 70.75 \\
\hline & Predicted & N/D & N/D & N/D & 35.8 & $\mathrm{~N} / \mathrm{D}$ & 57.2 & 61.52 & N/D \\
\hline & Difference & N/D & N/D & N/D & 1.05 & N/D & 3.2 & 2.48 & N/D \\
\hline \multirow{3}{*}{18} & Actual & 6.75 & 18.25 & 24 & 34.75 & 42 & 54 & 64 & 70.75 \\
\hline & Predicted & N/D & 21.08 & $\mathrm{~N} / \mathrm{D}$ & 31.46 & 41.84 & 57.08 & 61.58 & N/D \\
\hline & Difference & N/D & 2.83 & N/D & 3.29 & 0.16 & 3.08 & 2.42 & N/D \\
\hline \multirow{3}{*}{19} & Actual & 6.75 & 18.25 & 24 & 34.75 & 42 & 54 & 64 & 70.75 \\
\hline & Predicted & N/D & N/D & N/D & 37.5 & $\mathrm{~N} / \mathrm{D}$ & 56.1 & $\mathrm{~N} / \mathrm{D}$ & N/D \\
\hline & Difference & N/D & N/D & N/D & 2.75 & N/D & 2.1 & N/D & N/D \\
\hline
\end{tabular}

*N/D - not detectable 


\section{Development of Algorithms for Determination of Rock Properties}

Table 18 Predicted and actual locations of interfaces on Block 3

\begin{tabular}{|c|c|c|c|c|c|c|c|c|c|}
\hline Hole No. & Location (inch) & if1 & if 2 & if3 & if4 & if5 & if6 & if7 & if8 \\
\hline \multirow{3}{*}{2} & Actual & 4 & 16.5 & 25.75 & 31 & 44 & 50 & 62.375 & 68.375 \\
\hline & Predicted & N/D* & 18.51 & $\mathrm{~N} / \mathrm{D}$ & N/D & $\mathrm{N} / \mathrm{D}$ & 52.8 & 58.63 & 67.91 \\
\hline & Difference & N/D & 2.01 & N/D & N/D & N/D & 2.8 & 3.745 & 0.465 \\
\hline \multirow{3}{*}{4} & Actual & 4 & 16.5 & 25.75 & 31 & 44 & 50 & 62.375 & 68.375 \\
\hline & Predicted & N/D & 19.09 & $\mathrm{~N} / \mathrm{D}$ & $\mathrm{N} / \mathrm{D}$ & $\mathrm{N} / \mathrm{D}$ & 56.25 & 58.98 & $\mathrm{~N} / \mathrm{D}$ \\
\hline & Difference & N/D & 2.59 & N/D & N/D & N/D & 6.25 & 3.395 & N/D \\
\hline \multirow{3}{*}{5} & Actual & 4 & 16.5 & 25.75 & 31 & 44 & 50 & 62.375 & 68.375 \\
\hline & Predicted & N/D & 18.98 & $\mathrm{~N} / \mathrm{D}$ & $\mathrm{N} / \mathrm{D}$ & $\mathrm{N} / \mathrm{D}$ & 52.88 & 61.19 & 67.71 \\
\hline & Difference & N/D & 2.48 & N/D & N/D & N/D & 2.88 & 1.185 & 0.665 \\
\hline \multirow{3}{*}{6} & Actual & 4 & 16.5 & 25.75 & 31 & 44 & 50 & 62.375 & 68.375 \\
\hline & Predicted & N/D & $\mathrm{N} / \mathrm{D}$ & $\mathrm{N} / \mathrm{D}$ & $\mathrm{N} / \mathrm{D}$ & $\mathrm{N} / \mathrm{D}$ & $\mathrm{N} / \mathrm{D}$ & 62.93 & 70.425 \\
\hline & Difference & N/D & N/D & N/D & N/D & N/D & N/D & 0.555 & 2.05 \\
\hline \multirow{3}{*}{7} & Actual & 4 & 16.5 & 25.75 & 31 & 44 & 50 & 62.375 & 68.375 \\
\hline & Predicted & N/D & 18.41 & N/D & N/D & N/D & 52.5 & 59.655 & N/D \\
\hline & Difference & N/D & 1.91 & N/D & N/D & N/D & 2.5 & 2.72 & N/D \\
\hline \multirow{3}{*}{8} & Actual & 4 & 16.5 & 25.75 & 31 & 44 & 50 & 62.375 & 68.375 \\
\hline & Predicted & N/D & 15.75 & N/D & N/D & N/D & 54.47 & 58.32 & 68.905 \\
\hline & Difference & N/D & 0.75 & N/D & N/D & N/D & 4.47 & 4.055 & 3.625 \\
\hline \multirow{3}{*}{9} & Actual & 4 & 16.5 & 25.75 & 31 & 44 & 50 & 62.375 & 68.375 \\
\hline & Predicted & 5.83 & 18.51 & $\mathrm{~N} / \mathrm{D}$ & N/D & N/D & N/D & 59.17 & 68.0 \\
\hline & Difference & 1.83 & 2.01 & N/D & N/D & N/D & N/D & 3.205 & 0.375 \\
\hline \multirow{3}{*}{10} & Actual & 4 & 16.5 & 25.75 & 31 & 44 & 50 & 62.375 & 68.375 \\
\hline & Predicted & 5.45 & 19.09 & N/D & N/D & N/D & 53.52 & 57.95 & 68.31 \\
\hline & Difference & 1.45 & 2.59 & N/D & N/D & N/D & 3.52 & 1.675 & 0.065 \\
\hline \multirow{3}{*}{11} & Actual & 4 & 16.5 & 25.75 & 31 & 44 & 50 & 62.375 & 68.375 \\
\hline & Predicted & N/D & N/D & N/D & N/D & N/D & N/D & 62.93 & 70.425 \\
\hline & Difference & N/D & N/D & N/D & N/D & N/D & N/D & 0.555 & 2.05 \\
\hline
\end{tabular}

*N/D - not detectable 


\section{Development of Algorithms for Determination of Rock Properties}

Table 19 Predicted and actual locations of interfaces on Block 4

\begin{tabular}{|c|c|c|c|c|c|c|c|c|c|}
\hline Hole No. & Location (inch) & if1 & if 2 & if3 & if4 & if5 & if6 & if7 & if8 \\
\hline \multirow{3}{*}{23} & Actual & 7 & 16 & 25 & 33.75 & 44 & 50 & 61.75 & 68.25 \\
\hline & Predicted & N/D* & 13.635 & N/D & 33.725 & 44.425 & 50.73 & N/D & N/D \\
\hline & Difference & N/D & 2.365 & N/D & 0.025 & 0.425 & 0.73 & N/D & N/D \\
\hline \multirow{3}{*}{24} & Actual & 7 & 16 & 25 & 33.75 & 44 & 50 & 61.75 & 68.25 \\
\hline & Predicted & $\mathrm{N} / \mathrm{D}$ & $\mathrm{N} / \mathrm{D}$ & 24.91 & 31.34 & 42.41 & 49.23 & $\mathrm{~N} / \mathrm{D}$ & $\mathrm{N} / \mathrm{D}$ \\
\hline & Difference & N/D & N/D & 0.09 & 2.41 & 1.59 & 0.77 & N/D & N/D \\
\hline \multirow{3}{*}{25} & Actual & 7 & 16 & 25 & 33.75 & 44 & 50 & 61.75 & 68.25 \\
\hline & Predicted & N/D & N/D & 28.67 & 38.0 & N/D & 52.33 & $\mathrm{~N} / \mathrm{D}$ & N/D \\
\hline & Difference & N/D & N/D & 3.67 & 4.25 & N/D & 2.33 & N/D & N/D \\
\hline \multirow{3}{*}{26} & Actual & 7 & 16 & 25 & 33.75 & 44 & 50 & 61.75 & 68.25 \\
\hline & Predicted & N/D & N/D & 26.49 & 33.86 & 46.28 & 48.17 & $\mathrm{~N} / \mathrm{D}$ & N/D \\
\hline & Difference & N/D & N/D & 1.59 & 0.11 & 2.28 & 1.83 & N/D & N/D \\
\hline \multirow{3}{*}{27} & Actual & 7 & 16 & 25 & 33.75 & 44 & 50 & 61.75 & 68.25 \\
\hline & Predicted & $\mathrm{N} / \mathrm{D}$ & $\mathrm{N} / \mathrm{D}$ & 27.97 & 33.25 & $\mathrm{~N} / \mathrm{D}$ & 49.72 & 54.69 & $\mathrm{~N} / \mathrm{D}$ \\
\hline & Difference & N/D & N/D & 2.97 & 0.5 & N/D & 0.28 & 7.06 & N/D \\
\hline \multirow{3}{*}{28} & Actual & 7 & 16 & 25 & 33.75 & 44 & 50 & 61.75 & 68.25 \\
\hline & Predicted & N/D & N/D & N/D & 37.39 & 44.63 & 51.46 & $\mathrm{~N} / \mathrm{D}$ & N/D \\
\hline & Difference & N/D & N/D & N/D & 0.36 & 0.63 & 1.46 & N/D & N/D \\
\hline \multirow{3}{*}{29} & Actual & 7 & 16 & 25 & 33.75 & 44 & 50 & 61.75 & 68.25 \\
\hline & Predicted & $\mathrm{N} / \mathrm{D}$ & $\mathrm{N} / \mathrm{D}$ & 26.49 & 35.615 & 47.39 & 52.69 & 61.81 & $\mathrm{~N} / \mathrm{D}$ \\
\hline & Difference & N/D & N/D & 1.49 & 1.865 & 3.39 & 2.69 & 0.06 & N/D \\
\hline \multirow{3}{*}{30} & Actual & 7 & 16 & 25 & 33.75 & 44 & 50 & 61.75 & 68.25 \\
\hline & Predicted & N/D & 13.635 & $\mathrm{~N} / \mathrm{D}$ & 33.725 & 44.425 & 50.73 & $\mathrm{~N} / \mathrm{D}$ & $\mathrm{N} / \mathrm{D}$ \\
\hline & Difference & N/D & 2.365 & N/D & 0.025 & 0.425 & 0.73 & N/D & N/D \\
\hline \multirow{3}{*}{31} & Actual & 7 & 16 & 25 & 33.75 & 44 & 50 & 61.75 & 68.25 \\
\hline & Predicted & N/D & $\mathrm{N} / \mathrm{D}$ & 28.845 & 34.44 & 48.27 & 52.39 & N/D & N/D \\
\hline & Difference & N/D & N/D & 3.845 & 0.69 & 4.27 & 2.39 & N/D & N/D \\
\hline \multirow{3}{*}{32} & Actual & 7 & 16 & 25 & 33.75 & 44 & 50 & 61.75 & 68.25 \\
\hline & Predicted & N/D & N/D & 26.39 & 31.67 & 47.21 & 51.61 & 59.53 & N/D \\
\hline & Difference & N/D & N/D & 1.39 & 2.165 & 3.21 & 1.61 & 2.22 & N/D \\
\hline \multirow{3}{*}{33} & Actual & 7 & 16 & 25 & 33.75 & 44 & 50 & 61.75 & 68.25 \\
\hline & Predicted & $\mathrm{N} / \mathrm{D}$ & N/D & 25.71 & 34.375 & 46.8 & 51.71 & 59.08 & N/D \\
\hline & Difference & N/D & N/D & 0.71 & 0.625 & 2.8 & 1.71 & 2.67 & N/D \\
\hline
\end{tabular}

*N/D - not detectable

The detailed predicted and actual locations of interfaces between the embedded rock layers in Blocks 2, 3 and 4 are shown in Tables 17, 18 and 19. For the twenty-six drilled 


\section{Development of Algorithms for Determination of Rock Properties}

holes on the three blocks, there are $208(26 \times 8)$ interfaces drilled through and 113 of them, up to $54.33 \%$, are not detectable. Here, not-detectable means no outliers detected near the interface between two adjacent rock layers. Seventy-two out of ninety-five predicted interfaces, up to $26.87 \%$, have errors less than 3.0 inches compared to the actual locations of the interfaces between the rock layers. If looking at the distribution of drilling hardness for holes drilled on Block 4 (Figures II-16 to II-26, Appendix II), it can be seen that most of the fluctuations happened on argillite and white marble. The reason probably is that these two rock layers are so strong and it needs time to build up hydraulic pressure to maintain the pre-set penetration rate and rotation rate.

In addition, there are some unexpected interfaces, located at 48.00 inches for Hole 18, 72.66 inches for Hole 2, 72.07 inches for Hole 8, 72.2 inches for Hole 9, 76.11 inches for Hole 11, 51.81 inches for Hole 26, 37.6 inches for Hole 27, and 35.48 inches for Hole 32, are detected respectively. All the unexpected interfaces were detected within concrete layer except that the one detected from Hole 18 was in brown sandstone. Since the working mechanism of the hydraulic system with this control mode has not been comprehensively understood, it is not conclusive whether it was caused by the variation of rock properties or the adjustment of the hydraulic system; thus these extra interfaces detected in this test were not taken into account in this analysis.

It can be seen from the analysis results in drilling hardness obtained from the three drilled blocks that the reliable predicted locations of the interfaces between the rock layers, which have errors less than 3.0 inches compared to the actual locations, are only $27.87 \%$. From geological identification point of view, this result is not acceptable.

\subsubsection{Test Results for Fractured Block}

The Fractured Block was drilled by considering the thrust cap and the control mode is constant penetration rate and rotation rate simultaneously (Table 15). Three concrete layers with the same UCS were embedded into this block (Figure 50). 


\section{Development of Algorithms for Determination of Rock Properties}

Table 20 Predicted and actual locations of fractures on Fractured Block

\begin{tabular}{|c|c|c|c|c|}
\hline Hole No. & Location (inch) & f1 & $\mathrm{f} 2$ & f3 \\
\hline \multirow{3}{*}{1} & Actual & 15.0 & 30.0 & 45.0 \\
\hline & Predicted & N/D* & $\mathrm{N} / \mathrm{D}$ & N/D \\
\hline & Difference & N/D & N/D & N/D \\
\hline \multirow{3}{*}{2} & Actual & 15.0 & 30.0 & 45.0 \\
\hline & Predicted & N/D & $\mathrm{N} / \mathrm{D}$ & 44.52 \\
\hline & Difference & N/D & N/D & 0.48 \\
\hline \multirow{3}{*}{3} & Actual & 15.0 & 30.0 & 45.0 \\
\hline & Predicted & $\mathrm{N} / \mathrm{D}$ & $\mathrm{N} / \mathrm{D}$ & 45.54 \\
\hline & Difference & N/D & N/D & 0.54 \\
\hline \multirow{3}{*}{4} & Actual & 15.0 & 30.0 & 45.0 \\
\hline & Predicted & $\mathrm{N} / \mathrm{D}$ & 29.41 & 47.57 \\
\hline & Difference & N/D & 0.59 & 0.175 \\
\hline \multirow{3}{*}{5} & Actual & 15.0 & 30.0 & 45.0 \\
\hline & Predicted & 14.32 & N/D & N/D \\
\hline & Difference & 0.68 & N/D & N/D \\
\hline \multirow{3}{*}{6} & Actual & 15.0 & 30.0 & 45.0 \\
\hline & Predicted & 14.25 & $\mathrm{~N} / \mathrm{D}$ & N/D \\
\hline & Difference & 0.75 & N/D & N/D \\
\hline \multirow{3}{*}{7} & Actual & 15.0 & 30.0 & 45.0 \\
\hline & Predicted & N/D & 32.3 & 44.98 \\
\hline & Difference & N/D & 2.3 & 0.02 \\
\hline
\end{tabular}

*N/D - not detectable

The detailed predicted and actual locations of the fractures between the embedded concrete layers in the Fractured Block are shown in Table 20. For the seven drilled holes, there are $21(7 \times 3)$ fractures drilled through and 13 of them, up to $61.9 \%$, are not detectable. The errors between the detected and actual fracture locations are less than 3 inches. In addition, there are some unexpected discontinuities were detected from Holes 4 (at 36.54 and 40.10 inches) and 7 (at 40.76 and 49.55 inches). Since this Section is going to compare which controlling parameter could yield better prediction result, the fewer detected fractures and unexpectedly detected interfaces on this Fractured Block showed that this control mode, i.e. controlling penetration rate and rotation rate simultaneously can not achieve the goal in identifying the locations of fractures. 


\section{Development of Algorithms for Determination of Rock Properties}

\subsubsection{Test Result for Solid Block}

The Solid Block (Figure 51) was drilled by considering the thrust cap and the control mode is also constant penetration rate and rotation rate simultaneously (Table 16).

Holes 28 to 33 were drilled with 1-3/8-in bit, and Holes 34 to 39 were drilled with 11/32-in bit. Holes 28 to 33 (Figures II-34 to II- 45, Appendix II) basically show the same variation patterns, drilling hardness keeps increasing till 20 inches, and then becomes more consistent. Figures II-34 to II 45 show that there are at least one discontinuity derived from the drilling hardness. As explained in Section 4.2, the reason for the unexpected discontinuities s that if a sub-layer is stronger or weaker than its adjacent rock, the corresponding drilling hardness should be higher or lower than its adjacent rock. Thus, the corresponding discontinuity or interface will be detected since the Solid Block is not completely uniform in strength and particle distribution.

\subsubsection{Test Result for Block 6 (Layered Block)}

The test results for Block 6 were presented in Section 4.2 (Table 14 and Figures 32 to 40). As mentioned in Section 4.2, the maximum, minimum and average errors between the predicted and actual location of interface are 2.57, 0.06 and 1.025 inches respectively. Thirty predicted interfaces up to $72.5 \%$ have errors less than 3.0 inches. It seems that the results obtained from these holes are better and can basically identify the locations of the interfaces except holes 1 and 2 in which the thrust cap were set lower than other holes drilled (Table 13) and penetration rate and rotation rate were controlled simultaneously. Holes 5, 6, 7, and 8, in which the penetration rate or rotation rate were allowed free, have better results in identifying the locations of the interfaces (Figures 39, 40, 41, and 42) and the distribution of the drilling hardness is more consistent within one rock layer.

\subsubsection{Summary of the Influences of the Pre-settings before Drilling}

Although the working mechanisms of the hydraulic system with different control modes have not been comprehensively understood, the analysis results of the laboratory 


\section{Development of Algorithms for Determination of Rock Properties}

tests conducted on Blocks 2, 3, and 4, Fractured Block, Solid Block and Block 6 did provide some insight into possible improvements in current research. Blocks 2, 3, and 4 yielded the worst prediction result, only 95 out of 208 interfaces up to $45.67 \%$ were detected, and only $26.87 \%$ of the detected interfaces had errors less than 3.0 inches. There were 8 out of 21 fractures up to $38.1 \%$ detected from the Fractured Block and all the detected interfaces had errors less than 1.0 inches. In addition, the distribution of drilling hardness for the holes drilled in the Fractured Block and Solid Block is more consistent than that for the holes drilled in Blocks 2, 3 and 4. For Block 6, 30 out of 40 interfaces up to $75 \%$ were detected and $72.5 \%$ of them had errors less than 3 inches. This is big improvement in prediction percentage and accuracy. The big difference for Block6 from other drilled Blocks in controlling parameters is that thrust cap was considered and allowed either penetration rate or rotation rate free during drilling (for the tests conducted on Block 6), which eventually yields better results. Comparatively, the tests conducted on Blocks 2, 3, and 4 just used constant penetration rate and rotation rate and there was nothing to do with thrust before drilling. Thus the drilling data yielded worse result in identifying the locations of interfaces. The major reason is probably that the control modes (1) (2) could cause less adjustment time for the hydraulic system, which can effectively build up or reduce hydraulic pressure in order to maintain the controlled parameters.

In summary, it seems that using control modes (1) and (2) can get much better results higher prediction percentage and better accuracy (See the results from the layered block (Block 6) drilled in Jan. 2002) in identifying the locations of interfaces and showing evident differences in drilling hardness of rock layers. In other words, pre-setting higher thrust cap, and allowing either penetration rate or rotation rate free (not fixed simultaneously) can yield much better results in identifying the geological variations.

\subsection{Classification of Rock Type}

Rock type can give an experienced geological or mining engineer a basic recognition and understanding about how strong the rock is, or how large the average compressive 


\section{Development of Algorithms for Determination of Rock Properties}

strength is. Some experienced geological or mining engineers can recognize a rock type based on the color, particle size, and the distribution of fractures or bedding development of a rock sample. However, it is still impossible to exactly determine the rock type even if the compressive strength is known, because the same compressive strength may be shared by several different rock types. In this section, the supervised discriminant analysis, which is one of the technologies of the multivariate analysis, is employed to do rock type classification/prediction using the drilling parameters.

For a specific underground entry roof, the rock type of the embedded rock layers in the roof does not change much except the thickness and fracture distribution of the rock layers. These substantial features of rock allow the classification of rock types by first drilling several normal (roof bolting) drilling holes (at least 3 holes); thus defines the group data for each rock type in conjunction with the core log. Based on the group data, the training data, the group center, and the covariance matrix can be determined. The group center and the covariance matrix are used for the supervised discriminant analysis. In this specific drilling case, the supervised discriminant analysis, i.e., predictive discriminant analysis (PDA) is chosen to classify rock type.

The problem with the neural network when using it, as depicted in Section 2.5, is that there are no convincing methods to determine the learning rate and the number of hidden layers, although these two parameters play a vital role during the neural network training process. As a comparison, there are no such kinds of problems in using discriminant analysis to do pattern classification.

A decision or classification or assignment rule that is commonly used is based on the maximum likelihood principle ${ }^{[22]}$ : Assign a unit to the population in which its observation vector has the greatest likelihood of occurrence. This may be viewed in terms of likelihood function or density functions or probability functions. To simplify the discussion, consider a single (continuous) variable $\mathrm{X}$ and two populations. Suppose that (theoretical) models of the two populations of $X$ scores are represented as $f_{1}$ and $f_{2}$ that represent the two probability (density) functions. Given that the unit is randomly selected 


\section{Development of Algorithms for Determination of Rock Properties}

from population 1, the likelihood of an observation $\mathrm{X}=\mathrm{a}$ is denoted $\mathrm{f}(\mathrm{a})$. Applying the maximum likelihood principle, we arrive at the following rule: Assign a unit with $\mathrm{X}=\mathrm{a}$ to population 1 if $\mathrm{f}_{1}(\mathrm{a})>\mathrm{f}(\mathrm{a})$, that is, if the likelihood of an observation $\mathrm{X}=\mathrm{a}$ is greater for population 1 than for population 2; otherwise, assign the unit to population 2.

For a general multivariate g-group classification problem, it assumes that the 'form' of the density functions is the same for all $g$ groups or populations: for example, the $g$ groups are all multivariate normal. Let $\mathrm{f}$ denote this common density function, then the maximum likelihood rule is: Assign unit $\mathrm{u}$ to population $\mathrm{m}$ if the likelihood (probability) of the observation vector, $\mathrm{X}_{\mathrm{u}^{\prime}}$ is greater for group $\mathrm{m}$ than for any other group. This rule may be stated as follows ${ }^{[22]}$ :

Assign unit $\mathrm{u}$ to population $\mathrm{m}$ if

$$
f\left(X_{u} \mid m\right)>f\left(X_{u} \mid m^{\prime}\right) \text { for } \mathrm{m}^{\prime} \neq \mathrm{m}
$$

This rule may be stated in terms of something called inverse probability rather than in terms of likelihood. Such a probability is denoted as $\mathrm{P}(\mathrm{X} \mid \mathrm{m})$ (also called "typicality probability") and may be viewed as the proportion of units in population $\mathrm{m}$ that have score vectors "near" X.

Assign unit $\mathrm{u}$ to population $\mathrm{m}$ if

$$
P\left(X_{u} \mid m\right)>P\left(X_{u} \mid m^{\prime}\right) \quad \text { for } \mathrm{m}^{\prime} \neq \mathrm{m}
$$

Another view of the rule is taken by considering the probability of unit $\mathrm{u}$ belonging to group $g$ given that the unit has a particular observation vector, $\mathrm{X}_{\mathrm{u}}$. This probability, denoted by $\boldsymbol{P}\left(\boldsymbol{m} \mid \boldsymbol{X}_{u}\right)$, is called the posterior probability of membership in population $\mathrm{m}$, "posterior" in the sense that is a probability of population membership conditioned on 


\section{Development of Algorithms for Determination of Rock Properties}

knowing $\mathrm{X}_{\mathrm{u}}$, that is, after the $\mathrm{p} X$ values are obtained. Posterior probability can minimize the total number of misclassification errors. From a statistical point of view, predictive discriminant analysis (PDA) focus on the classification and/or the prediction of group membership (e.g. rock type) by comparing the posterior probability of the new data set being in different groups based on the Mahalanobis Distance between the new data set and different group centroids. The definition of posterior probability is that the probability of unit $u$ belonging to group $g$ given that the unit has a particular observation vector, $X_{\mathrm{u}}$.

In this specific drilling case, the covariance matrices of group data (drilling data sets) obtained from different rocks are unequal, thus the unequal quadratic form of posterior probability is applied (Eqs. 28 and 29) ${ }^{[22]}$.

Assign unit $\mathrm{u}$ to group $\mathrm{m}$ if

$$
P\left(m \mid X_{u}\right) \succ P\left(m^{\prime} \mid X_{u}\right) \text { for } \mathrm{m}^{\prime} \neq \mathrm{m}
$$

where $P\left(m \mid X_{u}\right)=\frac{P\left(X_{u} \mid m\right)}{\sum_{m^{\prime}=1}^{g} P\left(X_{u} \mid m^{\prime}\right)}=\frac{\left|S_{m}\right|^{-\frac{1}{2}} \exp \left(-\frac{1}{2} D_{u m}^{2}\right)}{\sum_{m^{\prime}=1}^{g}\left|S_{m^{\prime}}\right|^{-\frac{1}{2}} \exp \left(-\frac{1}{2} D_{u m^{2}}^{2}\right)}$

$\mathrm{S}_{\mathrm{m}}$ - the covariance matrix for group $\mathrm{m}$ (rock type $\mathrm{m}$ )

$\mathrm{D}_{\mathrm{um}}$ - mahalanobis distance

Suppose that there are g groups (m rock types) and one point represents a vector $\mathrm{X}_{\mathrm{u}}$ of $\mathrm{p}$ observations on an experimental unit and the other point represents a centroid for a group $\mathrm{m}$. The distance between $\mathrm{X}_{\mathrm{u}}$ (the observation vector for unit $\mathrm{u}$ ) and $\mu_{\mathrm{m}}$ (the centroid for group $\mathrm{m}$ ) can be represented by

$$
D_{u m}=\left[\left(X_{u}-\mu_{m}\right) S_{m}^{-1}\left(X_{u}-\mu_{m}\right)\right]^{\frac{1}{2}}
$$




\section{Development of Algorithms for Determination of Rock Properties}

where $\mathrm{S}_{\mathrm{m}}$ is the covariance matrix for group $\mathrm{m}$ (rock type $\mathrm{m}$ )

$$
S_{m}^{2}=\frac{1}{n_{m}-1} \sum_{m}\left(X-\overline{X_{m}}\right)^{\prime}\left(X-\overline{X_{m}}\right)
$$

where $\mathrm{X}$ - the vector of group $\mathrm{m}$

$\overline{X_{m}}$ - the mean vector of group $\mathrm{m}$

This distance index allows a unit to be classified as a member of the group that it is nearest. Unit $u$ is classified into group $m$ if $D_{\mathrm{lm}}$ is smaller than $\mathrm{D}_{\mathrm{um}}$ for all $\mathrm{m}^{\prime} \neq \mathrm{m}$ and $\mathrm{m}$, $\mathrm{m}^{\prime}=1,2,3, \ldots, \mathrm{g}$.

Equation 29 is a quadratic classification function, in other words, it is one type of nonlinear classification functions. The significant advantage of this function is that it does not need the group data to have an equal covariance matrix alike linear classification function.

Based on Eqs.29, 30 and 31, if there are $\mathrm{g}$ groups or $\mathrm{g}$ rock types, $\mathrm{g}$ posterior probabilities can be obtained. The group with the maximum posterior probability provides the best estimate of the correct rock type. Appendix III shows the procedure how to use predictive discriminative analysis (PDA) to do rock type classification. All the data used in this Section is from Block 6 since the applied control mode on this block yielded the best prediction results among all the laboratory tests.

\subsubsection{Determination of Variables Used in PDA}

Employing different number of variables used in the analysis of PDA may yield different hit rates, the major purpose of variables determination in this section is to determine the variables used in PDA. By using these determined variables, the hit rate in rock type classification can be considerately improved. In this section, five choices of determining variables are presumed (Table 21). These data are obtained from Block 6 


\section{Development of Algorithms for Determination of Rock Properties}

drilled in laboratory. The variables used in the final PDA analysis are determined in terms of the hit rates resulted from the five choices.

Table 21 Presumed choices of variable determination

\begin{tabular}{|c|c|c|c|c|c|}
\hline Choices & \multicolumn{5}{|c|}{ Variables used in each choice } \\
\hline Selection 1 & Drilling hardness & & & & \\
\hline Selection 2 & Thrust & Torque & & & \\
\hline Selection 3 & Drilling hardness & Thrust & Torque & & \\
\hline Selection 4 & Thrust & Torque & Penetration rate & rotation rate & \\
\hline Selection 5 & Drilling hardness & Thrust & Torque & Penetration rate & $\begin{array}{c}\text { rotation } \\
\text { rate }\end{array}$ \\
\hline
\end{tabular}

The comparison of classification and/or prediction with the five variable-selections is shown in Table 21 and the classification/prediction results for the eight holes are shown in Table 22. In this analysis, Holes 2, 3, 4, 6, and 7 were used to determine the group data, and Holes 1, 5, and 8 were used to predict. Based on Table 22, the number of original points and the number of classified or predicted points were reorganized in terms of the three types of rock layers drilled in the eight holes (Table 23).

It can be concluded from Tables 22 and 23 that Selection 5, using thrust, penetration rate, torque, rotation rate and drilling hardness to classify the rock type, can yield the highest hit rate when comparing the five variable-selections. Averagely, for the drilled three rock layers, $81.75 \%$ of original points from the concrete were classified, $78.75 \%$ of original points from the light brown sandstone were classified, and $76.25 \%$ original points from the argillite were classified (Table 23). Although the very high hit rates were obtained from concrete for each hole using Selection 1 and Selection 2, the hit rates from light brown sandstone and argillite were very low (see Table 22), except for hole 8. Selection 3 improved the hit rates slightly but it was not as high as Selection 4 and 5. Comparing the hit rates from Selection 4 with that from Selection 5, the hit rates obtained 


\section{Development of Algorithms for Determination of Rock Properties}

from Selection 5 were a little higher in most cases regardless of the hole data that were used for classification (holes 2, 3, 4, 6, 7) or prediction (holes 1, 5, 8).

The success of applying the PDA really depends on two factors, one is the chosen variables, and the second is the number of the variables. The chosen variables, to some extent, should indicate certain variations while rock properties change. All the five parameters (four original drilling parameters, one derived drilling parameter) used in the comparison show the variations more or less while drilling in different rock layers, although the variations in magnitude are different. From a statistical point of view, the drilling information can not be fully contained by just one, two, or three original or derived parameters. This is the reason why Selection 1, Selection 2, and Selection 3 obtained lower and unstable hit rates for each rock type in different holes. Although drilling hardness (Selection1) can demonstrate the differences in magnitude among different rock layers, there are still some variations that may overlap with other rock layers. The hit rate could be improved by the increasing of the number of variables (see Table 22), but it does not mean that unlimitedly increasing the number of variables in the PDA can yield higher hit rate. Discriminant analysis indicates that using fewer variables in the PDA can result in higher accuracy in classification ${ }^{[22]}$. From selections 1 to 5 , the number of variables increases from 1 to 5 , and the hit rates for each rock type basically keep increasing and being more stable. In selection 5, there is only one derived drilling parameter, drilling hardness. These five parameters can basically cover the drilling information. Even add one or two more derived parameter, which may not indicate the variations as clear as the drilling hardness while drilling different rock layers, the hit rate may not be improved significantly. This can be proved by the selection 4 and selection 5 .

Based on the analyses above, Selection 5, using thrust, penetration rate, torque and rotation rate to classify rock type, was chosen and applied in PDA. 


\section{Development of Algorithms for Determination of Rock Properties}

Table 22 Comparison of classification and/or prediction with the five variable-selections

\begin{tabular}{|c|c|c|c|c|c|c|c|}
\hline \multicolumn{3}{|c|}{ Drilled holes } & Selection 1 & Selection 2 & Selection 3 & Selection 4 & Selection 5 \\
\hline \multirow{9}{*}{ Hole 1} & \multirow{3}{*}{ Concrete } & Number of original points & 103 & 103 & 103 & 103 & 103 \\
\hline & & Number of classified points & 103 & 103 & 103 & 103 & 103 \\
\hline & & Hit rate $(\%)$ & 100 & 100 & 100 & 100 & 100 \\
\hline & \multirow{3}{*}{$\begin{array}{l}\text { Light brown } \\
\text { sandstone }\end{array}$} & Number of original points & 22 & 22 & 22 & 22 & 22 \\
\hline & & Number of classified points & 0 & 9 & 10 & 11 & 12 \\
\hline & & Hit rate $(\%)$ & $\mathbf{0}$ & 40.91 & 45.45 & 50 & 54.55 \\
\hline & \multirow{3}{*}{ Argillite } & Number of original points & 21 & 21 & 21 & 21 & 21 \\
\hline & & Number of classified points & 0 & 0 & 2 & 5 & 5 \\
\hline & & Hit rate $(\%)$ & $\mathbf{0}$ & $\mathbf{0}$ & 9.52 & 23.81 & 23.81 \\
\hline \multirow{9}{*}{ Hole 2} & \multirow{3}{*}{ Concrete } & Number of original points & 101 & 101 & 101 & 101 & 101 \\
\hline & & Number of classified points & 101 & 101 & 101 & 99 & 99 \\
\hline & & Hit rate (\%) & $\mathbf{0}$ & 100 & 100 & 98.02 & 98.02 \\
\hline & \multirow{3}{*}{$\begin{array}{l}\text { Light brown } \\
\text { sandstone }\end{array}$} & Number of original points & 22 & 22 & 22 & 22 & 22 \\
\hline & & Number of classified points & 0 & 9 & 10 & 11 & 12 \\
\hline & & Hit rate $(\%)$ & $\mathbf{0}$ & 40.91 & 45.45 & 50 & 54.55 \\
\hline & \multirow{3}{*}{ Argillite } & Number of original points & 23 & 23 & 23 & 23 & 23 \\
\hline & & Number of classified points & 0 & 0 & 0 & 13 & 12 \\
\hline & & Hit rate $(\%)$ & $\mathbf{0}$ & $\mathbf{0}$ & $\mathbf{0}$ & 56.52 & 52.17 \\
\hline \multirow{9}{*}{ Hole 3} & \multirow{3}{*}{ Concrete } & Number of original points & 96 & 96 & 96 & 96 & 96 \\
\hline & & Number of classified points & 96 & 77 & 75 & 51 & 54 \\
\hline & & Hit rate $(\%)$ & 100 & 80.21 & 78.13 & 53.13 & 56.25 \\
\hline & \multirow{3}{*}{$\begin{array}{l}\text { Light brown } \\
\text { sandstone }\end{array}$} & Number of original points & 20 & 20 & 20 & 20 & 20 \\
\hline & & Number of classified points & 0 & 10 & 20 & 20 & 20 \\
\hline & & Hit rate $(\%)$ & $\mathbf{0}$ & 50 & 100 & 100 & 100 \\
\hline & \multirow{3}{*}{ Argillite } & Number of original points & 20 & 20 & 20 & 20 & 20 \\
\hline & & Number of classified points & 0 & 14 & 10 & 20 & 20 \\
\hline & & Hit rate $(\%)$ & $\mathbf{0}$ & 70 & 50 & 100 & 100 \\
\hline \multirow{9}{*}{ Hole 4} & \multirow{3}{*}{ Concrete } & Number of original points & 88 & 88 & 88 & 88 & 88 \\
\hline & & Number of classified points & 88 & 69 & 71 & 48 & 56 \\
\hline & & Hit rate $(\%)$ & 100 & 78.41 & 8.068 & 54.55 & 63.64 \\
\hline & \multirow{3}{*}{$\begin{array}{l}\text { Light brown } \\
\text { sandstone }\end{array}$} & Number of original points & 20 & 20 & 20 & 20 & 20 \\
\hline & & Number of classified points & 9 & 8 & 18 & 17 & 17 \\
\hline & & Hit rate $(\%)$ & 45 & 40 & 90 & 85 & 85 \\
\hline & \multirow{3}{*}{ Argillite } & Number of original points & 19 & 19 & 19 & 19 & 19 \\
\hline & & Number of classified points & 0 & 9 & 6 & 19 & 18 \\
\hline & & Hit rate (\%) & $\mathbf{0}$ & 17.34 & 31.58 & 100 & 94.74 \\
\hline \multirow{9}{*}{ Hole 5} & \multirow{3}{*}{ Concrete } & Number of original points & 90 & 90 & 90 & 90 & 90 \\
\hline & & Number of classified points & 21 & 84 & 52 & 63 & 78 \\
\hline & & Hit rate $(\%)$ & 23.33 & 993.33 & $\begin{array}{lll}57.78 \\
\end{array}$ & 70 & 86.67 \\
\hline & \multirow{3}{*}{$\begin{array}{l}\text { Light brown } \\
\text { sandstone }\end{array}$} & Number of original points & 19 & 19 & 19 & 19 & 19 \\
\hline & & Number of classified points & 1 & 0 & 12 & 15 & 15 \\
\hline & & Hit rate $(\%)$ & 5.26 & $\mathbf{0}$ & 63.16 & 78.95 & 78.95 \\
\hline & \multirow{3}{*}{ Argillite } & Number of original points & 17 & 17 & 17 & 17 & 17 \\
\hline & & Number of classified points & 16 & 14 & 16 & 10 & 12 \\
\hline & & Hit rate $(\%)$ & 94.12 & 82.35 & 94.12 & $\begin{array}{l}58.82 \\
-12\end{array}$ & 70.59 \\
\hline Hole 6 & Concrete & Number of original points & 83 & 83 & 83 & 83 & 83 \\
\hline
\end{tabular}




\section{Development of Algorithms for Determination of Rock Properties}

\begin{tabular}{|c|c|c|c|c|c|c|c|}
\hline & & Number of classified points & 15 & 81 & 69 & 76 & 79 \\
\hline & & Hit rate $(\%)$ & 18.07 & 97.59 & 83.13 & 91.57 & 95.18 \\
\hline & \multirow{3}{*}{$\begin{array}{l}\text { Light brown } \\
\text { sandstone }\end{array}$} & Number of original points & 19 & 19 & 19 & 19 & 19 \\
\hline & & Number of classified points & 2 & 0 & 15 & 18 & 17 \\
\hline & & Hit rate $(\%)$ & 10.53 & $\mathbf{0}$ & 78.95 & 94.74 & 89.47 \\
\hline & \multirow{3}{*}{ Argillite } & Number of original points & 20 & 20 & 20 & 20 & 20 \\
\hline & & Number of classified points & 20 & 14 & 20 & 20 & 20 \\
\hline & & Hit rate $(\%)$ & 100 & 70 & 100 & 100 & 100 \\
\hline \multirow{9}{*}{ Hole 7} & \multirow{3}{*}{ Concrete } & Number of original points & 70 & 70 & 707 & 70 & 70 \\
\hline & & Number of classified points & 65 & 37 & 65 & 44 & 55 \\
\hline & & Hit rate $(\%)$ & 92.86 & 52.86 & 92.86 & 62.86 & 78.57 \\
\hline & \multirow{3}{*}{$\begin{array}{l}\text { Light brown } \\
\text { sandstone }\end{array}$} & Number of original points & 19 & 19 & 19 & 19 & 19 \\
\hline & & Number of classified points & 6 & 16 & 16 & 15 & 16 \\
\hline & & Hit rate $(\%)$ & 31.58 & 84.21 & 84.21 & 78.95 & 84.21 \\
\hline & \multirow{3}{*}{ Argillite } & Number of original points & 21 & 21 & 21 & 21 & 21 \\
\hline & & Number of classified points & 0 & 21 & 18 & 19 & 19 \\
\hline & & Hit rate $(\%)$ & $\mathbf{0}$ & 100 & 85.71 & 90.48 & 90.48 \\
\hline \multirow{9}{*}{ Hole 8} & \multirow{3}{*}{ Concrete } & Number of original points & 54 & 54 & 54 & 54 & 54 \\
\hline & & Number of classified points & 37 & 37 & 52 & 25 & 36 \\
\hline & & Hit rate $(\%)$ & 68.52 & 68.52 & 96.3 & 46.3 & 66.67 \\
\hline & \multirow{3}{*}{$\begin{array}{l}\text { Light brown } \\
\text { sandstone }\end{array}$} & Number of original points & 19 & 19 & 19 & 19 & 19 \\
\hline & & Number of classified points & 19 & 4 & 17 & 15 & 17 \\
\hline & & Hit rate (\%) & 100 & 21.05 & 89.47 & 78.95 & 89.47 \\
\hline & \multirow{3}{*}{ Argillite } & Number of original points & 19 & 19 & 19 & 19 & 19 \\
\hline & & Number of classified points & 12 & 18 & 16 & 18 & 16 \\
\hline & & Hit rate $(\%)$ & 63.16 & 94.74 & 84.21 & 94.74 & 84.21 \\
\hline
\end{tabular}

Table 23 Hit rate comparison among three rock types with the five variable-selections

\begin{tabular}{|c|c|c|c|c|c|c|c|}
\hline \multicolumn{2}{|c|}{ Drilled holes } & Recorded numbers of points & Selection 1 & Selection 2 & Selection 3 & Selection 4 & Selection 5 \\
\hline \multirow{9}{*}{$\begin{array}{c}8 \text { drilled } \\
\text { Holes }\end{array}$} & \multirow{3}{*}{ Concrete } & Number of original points & 685 & 685 & 685 & 685 & 685 \\
\hline & & Number of classified points & 526 & 589 & 588 & 509 & 560 \\
\hline & & Hit rate $(\%)$ & 76.79 & 85.98 & 85.84 & 74.31 & 81.75 \\
\hline & \multirow{3}{*}{$\begin{array}{c}\text { Light brown } \\
\text { sandstone }\end{array}$} & Number of original points & 160 & 160 & 160 & 160 & 160 \\
\hline & & Number of classified points & 37 & 56 & 118 & 122 & 126 \\
\hline & & Hit rate $(\%)$ & 23.125 & 35.0 & 73.75 & 76.25 & 78.75 \\
\hline & \multirow{3}{*}{ Argillite } & Number of original points & 160 & 160 & 160 & 160 & 160 \\
\hline & & Number of classified points & 48 & 100 & 88 & 124 & 122 \\
\hline & & Hit rate $(\%)$ & 30.0 & 62.5 & 55 & 77.5 & 76.25 \\
\hline
\end{tabular}




\section{Development of Algorithms for Determination of Rock Properties}

\subsubsection{Determination of Group Data and Covariance Matrix in PDA}

It is very important and indispensable to determine the group data and covariance matrix for each rock type in predictive discriminant analysis. The function of group data is to calculate the group center, covariance matrix. The covariance matrix is one of the most important components in calculating the Mahalanobis distance and posterior probability.

The determination of group data is very simple. In this analysis, each rock type has its own group data, which is defined by using the data sets extracted from five drilling holes. Assumed that the five matrixes, which compose of the drilling data sets with different size, of argillite obtained from the five holes were represented by sub-group $\mathrm{g}_{\mathrm{i}}$ $(\mathrm{i}=1,2,3,4,5)$, the group data $\mathrm{g}$ can be determined by gathering sub-groups $\mathrm{g}_{1}, \mathrm{~g}_{2}, \mathrm{~g}_{3}, \mathrm{~g}_{4}$, and $\mathrm{g}_{5}$ one by one, i.e. $\mathrm{g}=\left[\mathrm{g}_{1} ; \mathrm{g}_{2} ; \mathrm{g}_{3} ; \mathrm{g}_{4} ; \mathrm{g}_{5}\right]$.

In predictive discriminant analysis, each rock type has its own covariance matrix which can be determined either by $\mathrm{g}$ or $\mathrm{g}_{1}, \mathrm{~g}_{2}, \mathrm{~g}_{3}, \mathrm{~g}_{4}$, and $\mathrm{g}_{5} \mathrm{using}$ different algorithms. The covariance matrix determined by $\mathrm{g} 1, \mathrm{~g} 2, \mathrm{~g} 3, \mathrm{~g} 4$, and $\mathrm{g} 5$ can be pooled within-group covariance matrix $\mathrm{W}$ or between-groups matrix $\mathrm{B}$.

Determination of the covariance matrix using group data $\mathrm{g}$. The sample covariance between two variables $Y_{j}$ and $Y_{k}$ is defined by ${ }^{[29]}$ :

$\operatorname{cov}\left(Y_{j}, Y_{k}\right)=s_{j k}=\frac{\sum_{i}\left(y_{i j}-\overline{y_{j}}\right)\left(y_{i k}-\overline{y_{k}}\right)}{n-1}$

where $\mathrm{i}$ - the $\mathrm{i}^{\text {th }}$ sample

$\mathrm{n}$ - sample size 


\section{Development of Algorithms for Determination of Rock Properties}

In this drilling case, the group data consists of five variables determined in section 4.5.1, thrust, penetration rate, torque, rotation rate and drilling hardness. The covariance of the group data for rock $\mathrm{m}$ is given by:

$$
S_{m}=\left[\begin{array}{llll}
s_{1}^{2} & \mathrm{~s}_{12} & \ldots & \mathrm{s}_{15} \\
s_{12} & \mathrm{~s}_{2}^{2} & \cdots & \mathrm{s}_{25} \\
\vdots & \vdots & \ddots & \vdots \\
\mathrm{s}_{51} & \mathrm{~s}_{52} & \cdots & \mathrm{s}_{5}^{2}
\end{array}\right]=\left[s_{j k}\right]
$$

In an attempt to avoid such a case where the Mahalannobis distance is almost completely determined by one of the five variables ${ }^{[29]}, \mathrm{Y}_{\mathrm{j}}$, which has a large sample variance while other variables have small variances, all the five variables should be standardized. The distance is then computed on the matrix of standardized values. For this specific drilling case, the zscore scaling standardization technique was used. The algorithm is shown as:

$$
Y_{i k}^{\prime}=\left(Y_{i k}-\text { mean of variable } Y_{\mathrm{k}}\right) / \sigma
$$

where $Y_{i k}$ - the standardized value of the $i_{\mathrm{th}}$ data of variable $Y_{k}$

$Y_{i k}$ - the original value of the $i_{t h}$ data of variable $Y_{k}$

$\sigma$ - the sample standard deviation of variable $Y_{k}$

Determination of pooled within-group covariance matrix $\mathrm{W}$ and between-groups covariance matrix B. W and B can be determined by ${ }^{[29]}$

$$
\begin{aligned}
& W=\frac{1}{n-5} \cdot \sum_{i=1}^{5}\left(n_{i}-1\right) \cdot S_{i} \\
& B=\frac{1}{5-1} \cdot \sum_{i=1}^{5} n_{i} \cdot\left(x_{i_{-}} \text {mean }-x_{-} \text {mean }\right) \cdot\left(x_{i_{-}} \text {mean }-x_{-} \text {mean }\right)^{\prime}
\end{aligned}
$$




\section{Development of Algorithms for Determination of Rock Properties}

where $n_{i}$ - the sample size of the $i^{\text {th }}$ group, $g_{i}$

$\mathrm{n}-$ the total sample size of the five groups, $\mathrm{n}=\mathrm{n}_{1}+\mathrm{n}_{2}+\mathrm{n}_{3}+\mathrm{n}_{4}+\mathrm{n}_{5}$

$\mathrm{x}_{\mathrm{i} \_ \text {mean }}-$ the mean vector of the $\mathrm{i}^{\text {th }}$ group

$$
\mathrm{x} \_ \text {mean }- \text { the overall mean vector given by } \frac{\sum_{i=1}^{5} n_{i} \cdot x_{i} \text { mean }}{n}
$$

Table 17 shows the comparison of prediction results using different covariance matrixes calculated from Eqs. 32, 34, and 35. The test hole is Hole 8. When using the between-groups covariance matrix, all the data points were predicted as light brown sandstone. However, when using the pooled within-group covariance matrix, light brown sandstone could not be predicted and classified, and the hit rate of concrete was much lower than that obtained using Eq. 32.

As a result, Eq. 32 was used to calculate the covariance matrix based on the group data $\mathrm{g}$ that just gathers sub-groups $\mathrm{g}_{1}, \mathrm{~g}_{2}, \mathrm{~g}_{3}, \mathrm{~g}_{4}$, and $\mathrm{g}_{5}$ one by one. It can be expressed by the following matrix:

$$
g=\left[\begin{array}{l}
g_{1} \\
g_{2} \\
g_{3} \\
g_{4} \\
g_{5}
\end{array}\right]
$$




\section{Development of Algorithms for Determination of Rock Properties}

Table 24 Comparison of the prediction results using different covariance matrixes

\begin{tabular}{|c|c|c|c|c|c|}
\hline \multicolumn{3}{|c|}{ Drilled holes } & Using Eq.32 & Using Eq.34 & Using Eq.35 \\
\hline \multirow{9}{*}{ Hole 8} & \multirow{3}{*}{ Concrete } & Number of original points & 54 & 54 & 54 \\
\hline & & Number of classified points & 36 & 24 & 0 \\
\hline & & Hit rate (\%) & 66.67 & 44.44 & 0 \\
\hline & \multirow{3}{*}{$\begin{array}{l}\text { Light brown } \\
\text { sandstone }\end{array}$} & Number of original points & 19 & 19 & 19 \\
\hline & & Number of classified points & 17 & 0 & 19 \\
\hline & & Hit rate $(\%)$ & 89.47 & 0 & 100 \\
\hline & \multirow{3}{*}{ Argillite } & Number of original points & 19 & 19 & 19 \\
\hline & & Number of classified points & 16 & 17 & 0 \\
\hline & & Hit rate $(\%)$ & 84.21 & 89.47 & 0 \\
\hline
\end{tabular}




\section{Analysis on Field Testing Data}

Based on the laboratory tests, approaches for determining the locations of interfaces between rock layers and discontinuities and the rock type were developed. As shown in chapter 4, the derived parameters, drilling hardness and its slope, are capable of identifying variations in rock types and the location of interfaces among rock layers. In an effort to demonstrate their usefulness in field conditions, field tests were conducted at Mine A and Mine B.

\subsection{Test Conducted at Mine A}

\subsubsection{Test Site and Testing Parameters}

The arrangement of drilled holes is shown in Figure 52. Three holes were analyzed at test site 1 and four holes at test site 2 . In addition, a borehole was drilled near site 2 and a core log was obtained (Figure 53).

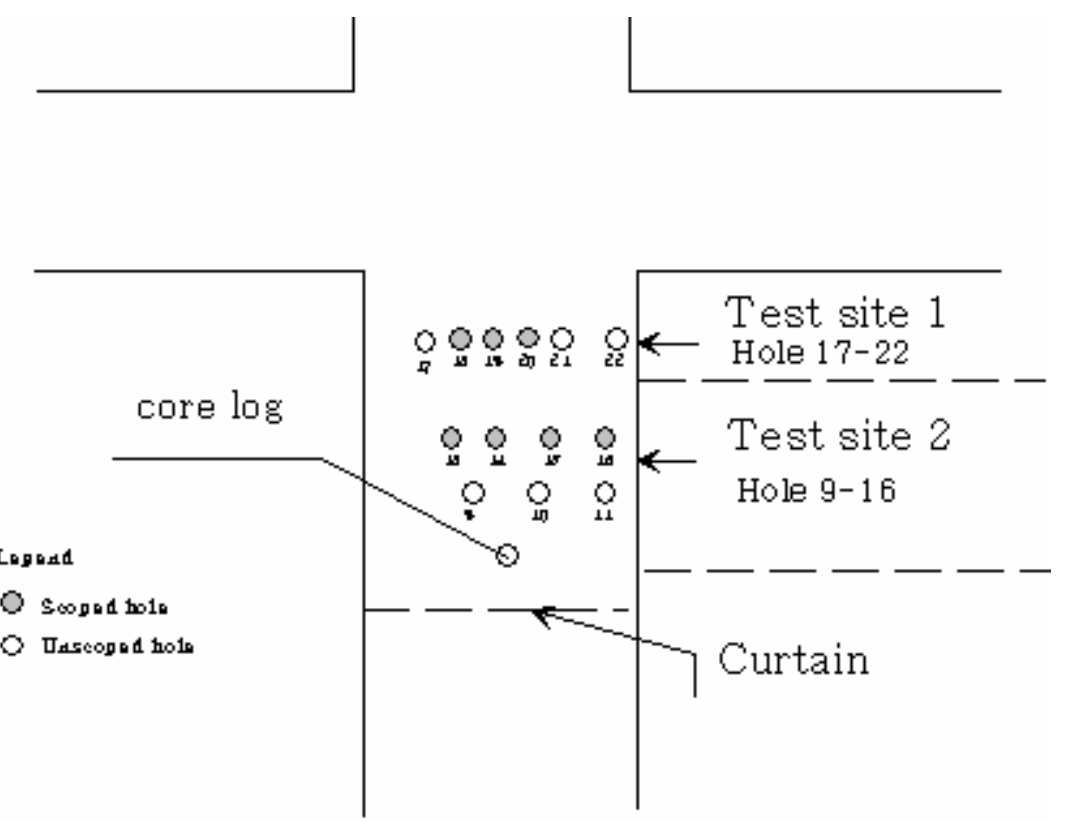

Figure 52 Testing site at Mine A 


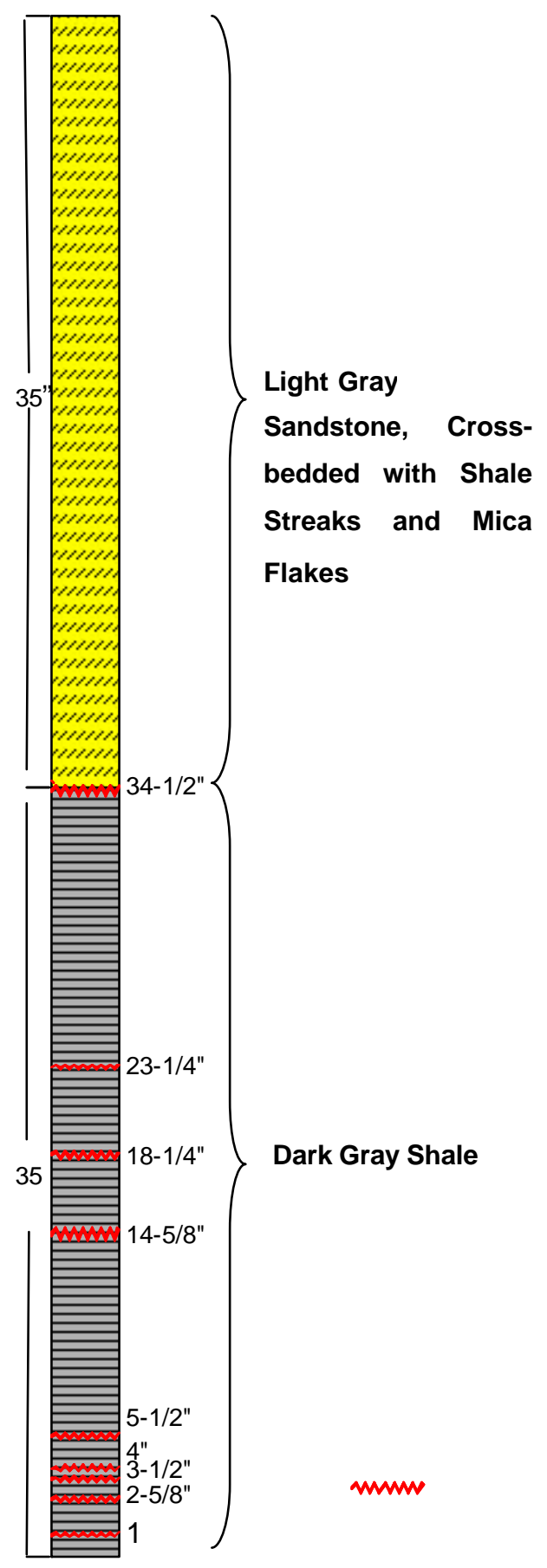

Figure 53 Core log near test site 2 


\section{Analysis on Field Testing Data}

The core $\log$ can be used to visually identify the roof geology within a certain area. However, it is too costly to drill many closely-spaced holes and cored them in order to map the roof geology. In an attempt to verify and improve the approach developed in the laboratory tests, a borehole camera scope system was used to obtain clear color images from the interior of a 1-3/8 inch diameter bolt hole (Figure 54). In this field test, 7 holes were scoped (holes 13, 14, 15, 16, 18, 19, 20).

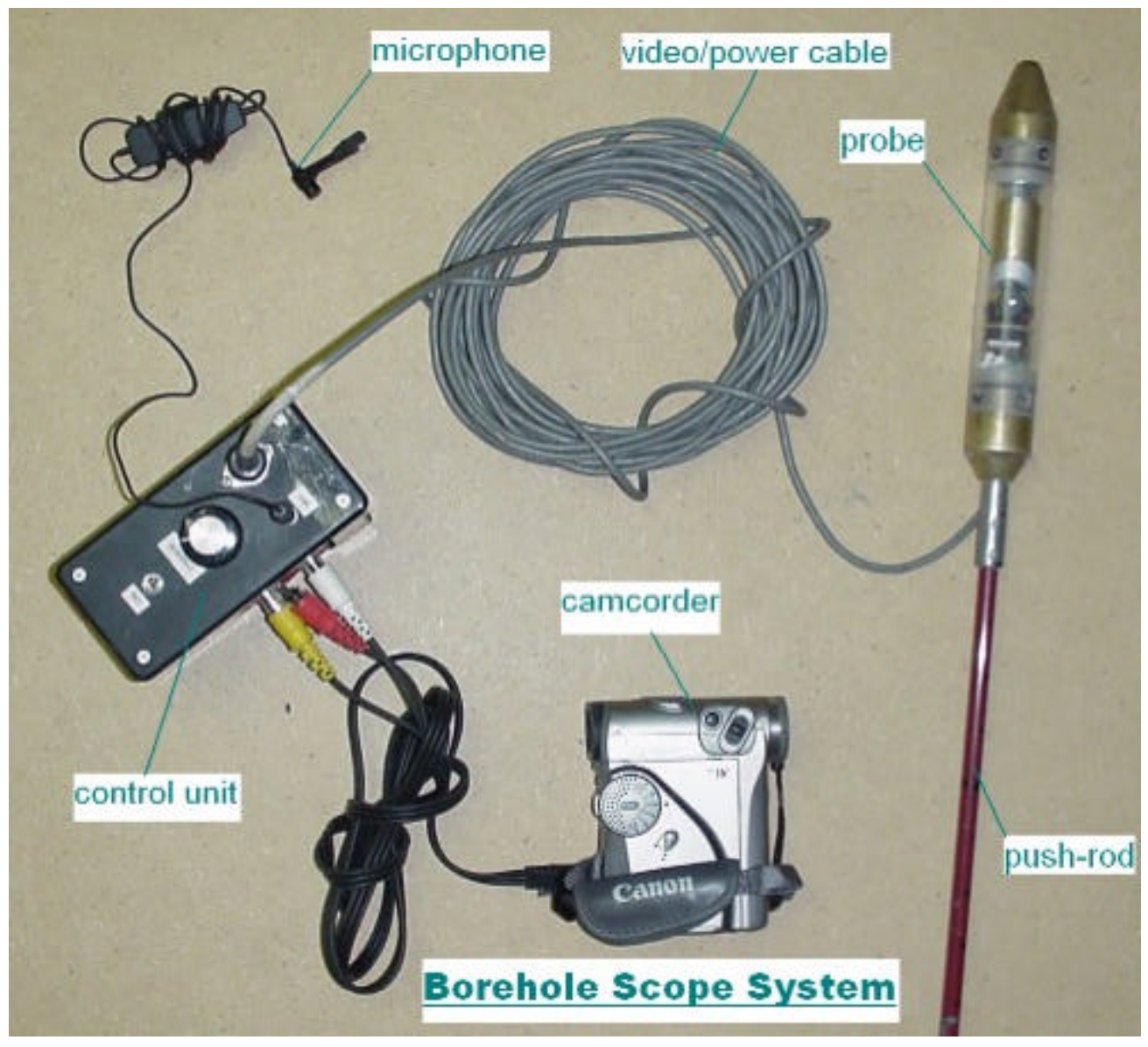

Figure 54 Borehole scope system 


\section{Analysis on Field Testing Data}

Table 25 Testing Parameters at Mine A

\begin{tabular}{|c|c|c|c|c|c|c|}
\hline Hole No. & $\begin{array}{c}\text { Bit size } \\
(\mathrm{inch})\end{array}$ & $\begin{array}{c}\text { P.R } \\
(\mathrm{in} / \mathrm{sec})\end{array}$ & $\begin{array}{c}\text { rotation rate } \\
(\text { Ro./min. })\end{array}$ & $\begin{array}{c}\text { Thrust cap } \\
(\mathrm{psi})\end{array}$ & $\begin{array}{c}\text { Hole Length } \\
\text { (in) }\end{array}$ & File Name \\
\hline 13 & $13 / 8$ & Free & 502 & 650 & 54 & 505 \\
\hline 14 & $13 / 8$ & Free & 502 & 650 & 53 & 506 \\
\hline 15 & $13 / 8$ & Free & 405 & 650 & 53 & 507 \\
\hline 16 & $13 / 8$ & Free & 405 & 650 & 52 & 508 \\
\hline $17^{*}$ & $13 / 8$ & 1.1 & Free & 650 & 53 & 512 \\
\hline 18 & $13 / 8$ & 0.8 & Free & 650 & 53 & 514 \\
\hline 19 & $13 / 8$ & 5.1 & 502 & 500 & 53.5 & 515 \\
\hline 20 & $13 / 8$ & 1.5 & Free & 650 & 55 & 517 \\
\hline
\end{tabular}

Legend: * - this hole was not scoped

Table 25 shows the values of these controlling parameters while drilling the scoped holes, including thrust cap, penetration rate and rotation rate.

\subsubsection{Process Results from Mine A}

Applying the same procedure used in the laboratory drilling tests, the field drilling data was used to calculate the drilling hardness and its slope by following the calculation procedure shown in Appendix I.

In this analysis, as described in Section 4.2, the features to be identified are divided into two types:

(1) Interface between two rock layers.

(2) Any kinds of discontinuities such as fracture, crack, etc. within one rock layer.

As mentioned in Section 4.2, the criteria for determining the location of an interface or a discontinuity is that if the peak value of the slope at location y is larger than or equal to the outer fence, y will be identified as the location of an interface. If the value of the negative slope at location $y$ is within the outer and inner fences, $y$ will be identified as the location of a discontinuity, regardless of whether it is a fracture or a crack. If there is more than one interface detected within 4 inches, the interface around that area can be determined by averaging the locations of the interfaces. In addition, it is necessary to 


\section{Analysis on Field Testing Data}

ignore the interfaces derived fom the first 5 inches (from the roof line) and the last 2 inches. All the algorithms for calculating the drilling hardness and its slope and determining the locations of interfaces and discontinuities were programmed with Matlab 6.0. The distributions of the drilling hardness and its slope along a drilled hole can be drawn automatically, and the locations of interfaces and discontinuities are output into a text file. Figure 55 shows an example of the text file (Hole 15). The left column shows either interface or discontinuity, and the right column shows the corresponding locations in inches.

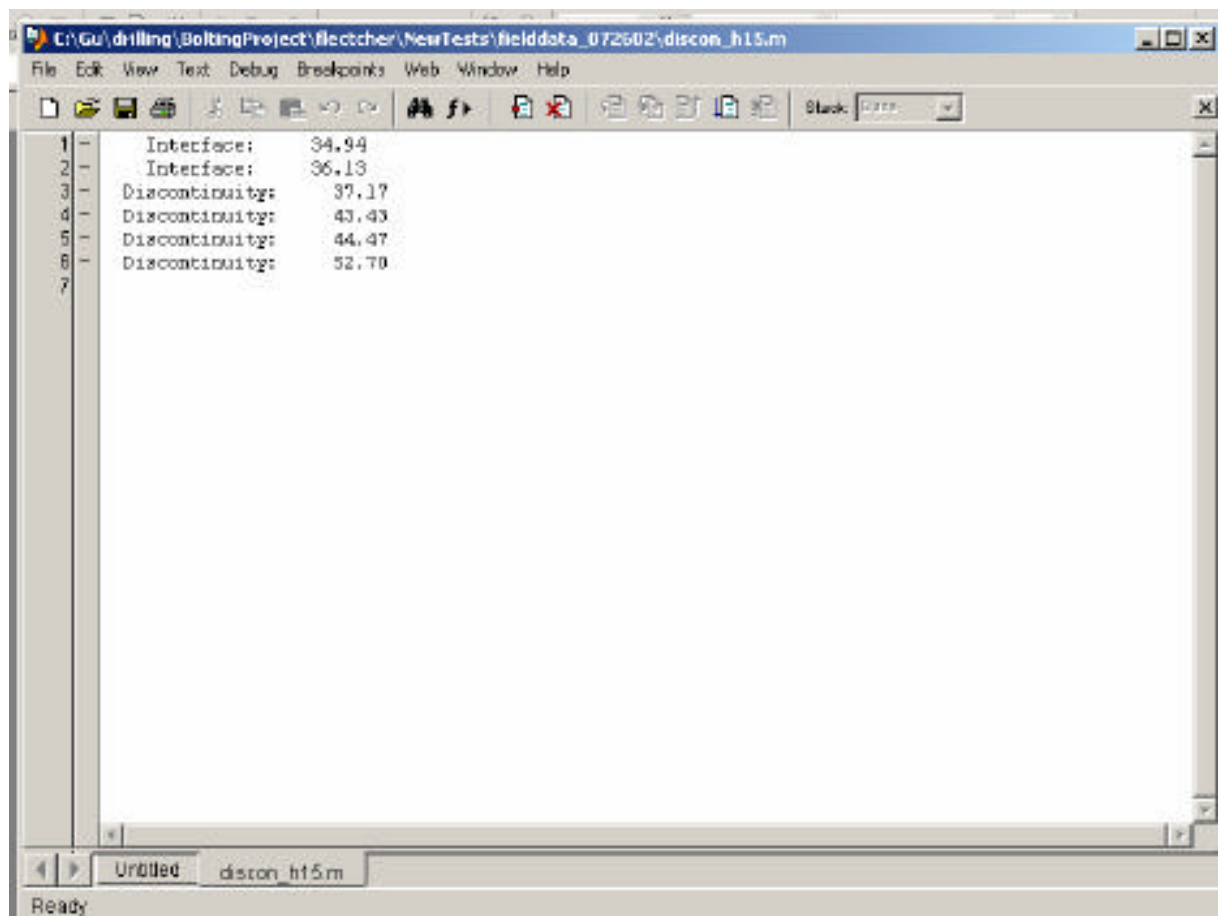

Figure 55 Output for locations of interfaces and discontinuities 


\section{Analysis on Field Testing Data}

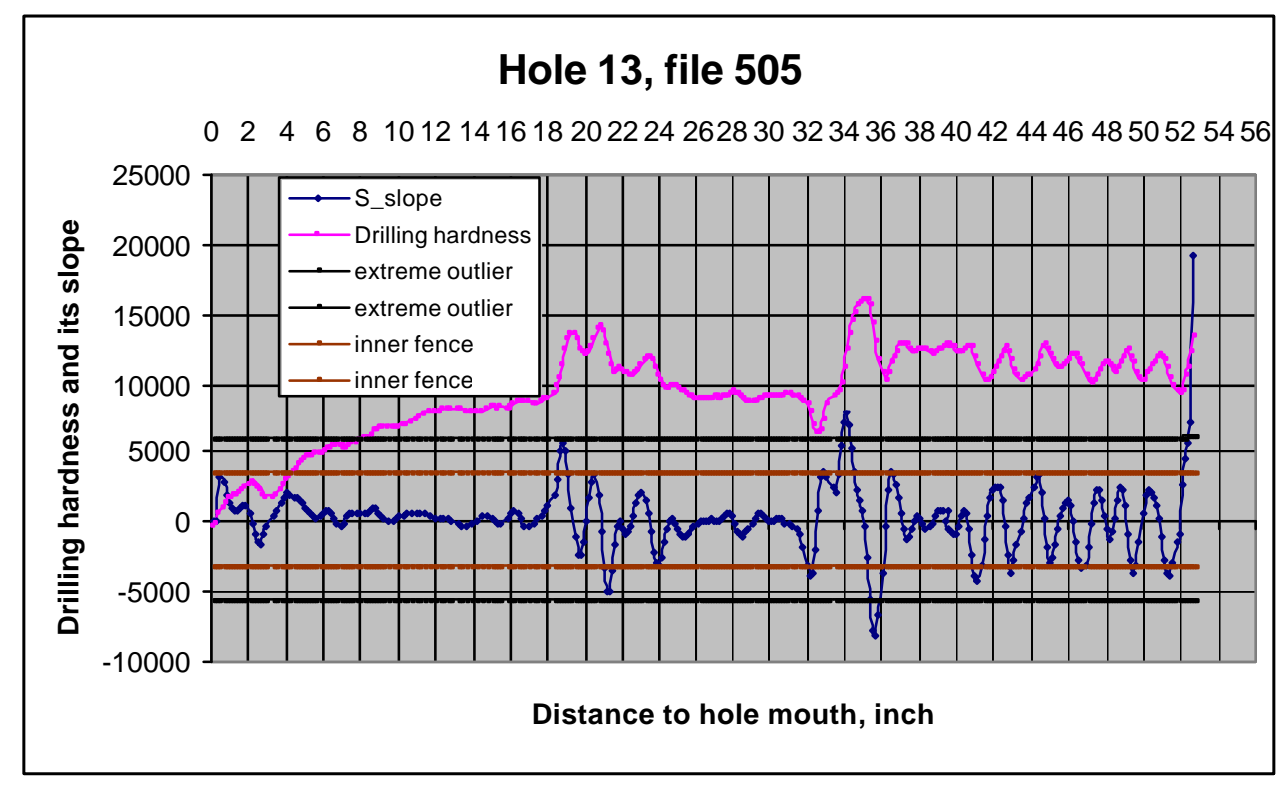

Figure 56 Drilling hardness and slope for Hole 13, Mine A

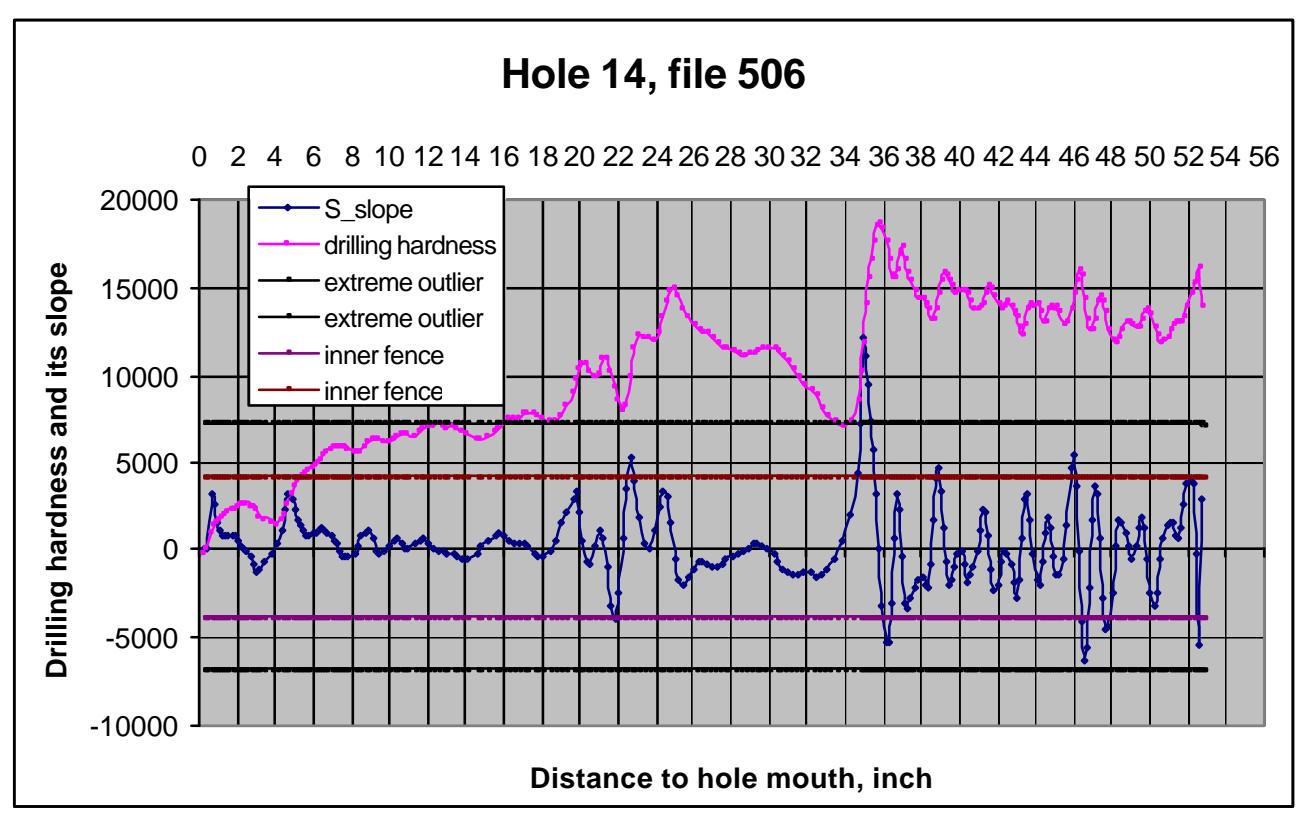

Figure 57 Drilling hardness and slope for Hole 14, Mine A 


\section{Analysis on Field Testing Data}

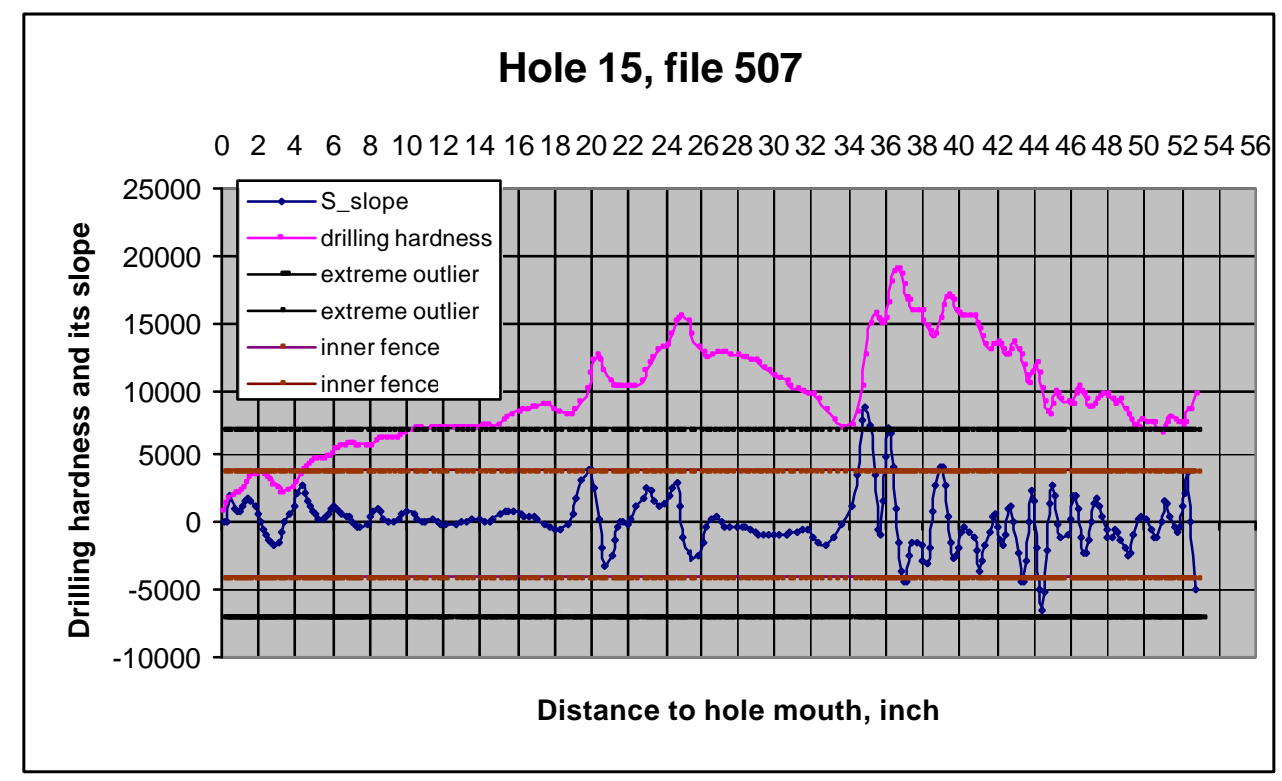

Figure 58 Drilling hardness and slope for Hole 15, Mine A

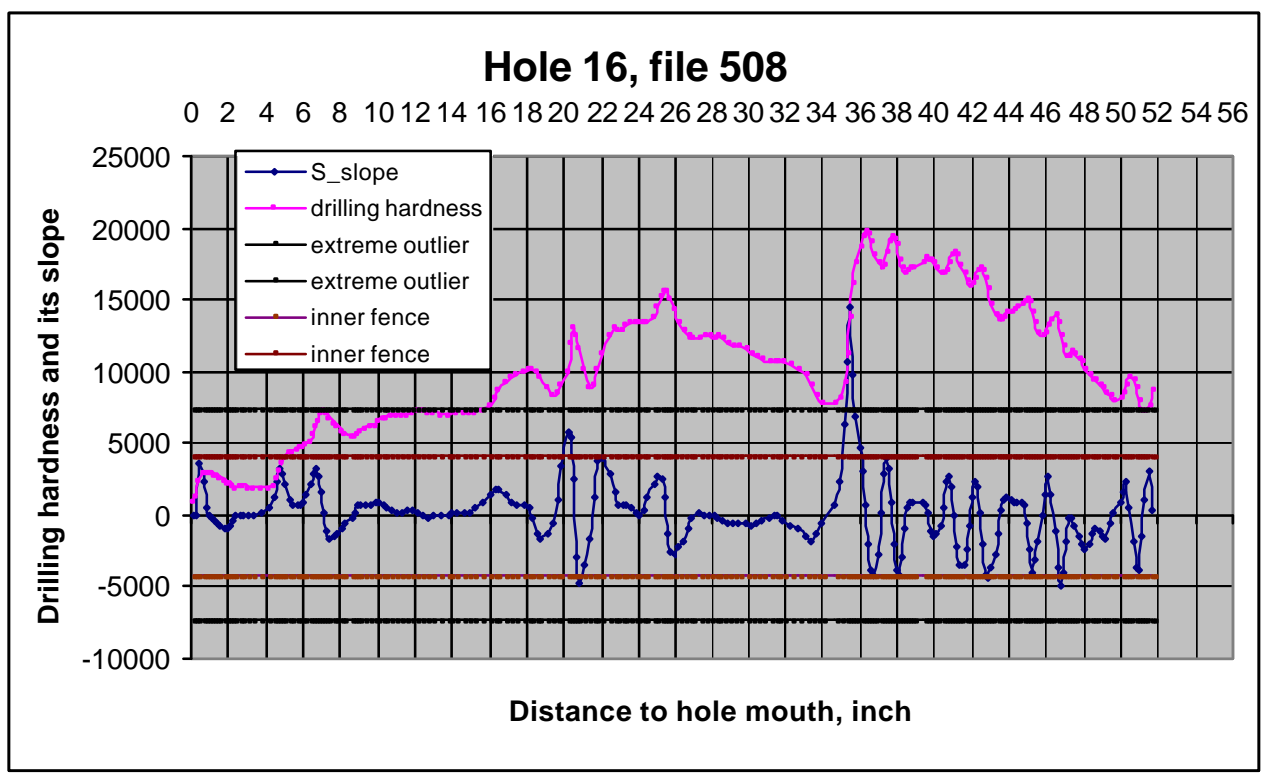

Figure 59 Drilling hardness and slope for Hole 16, Mine A 


\section{Analysis on Field Testing Data}

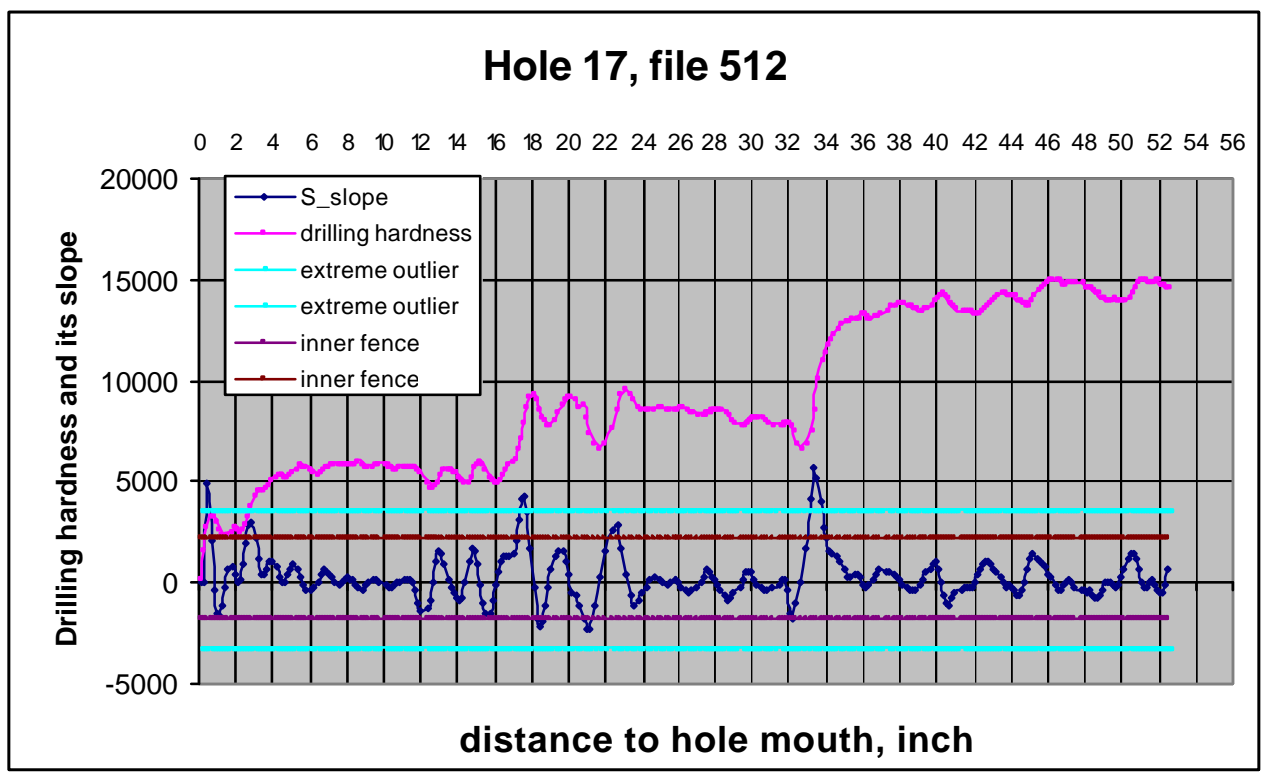

Figure 60 Drilling hardness and slope for Hole 17, Mine A

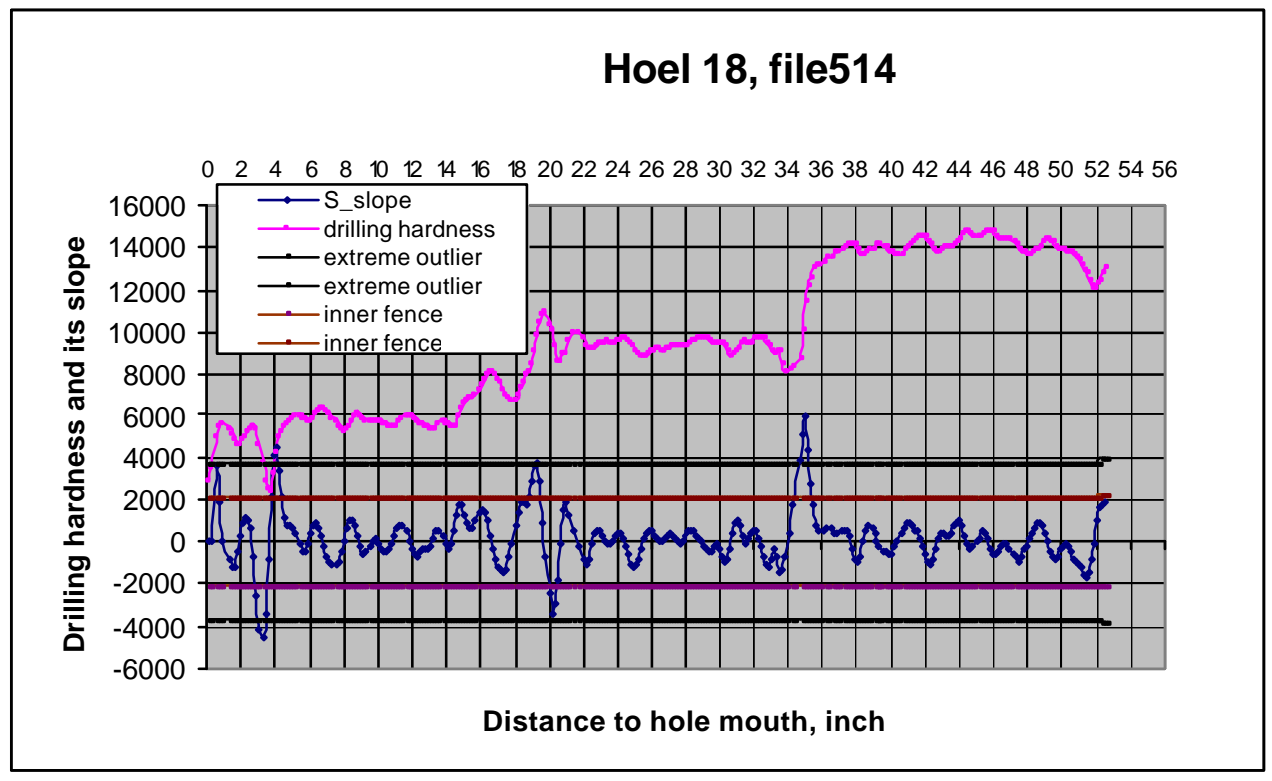

Figure 61 Drilling hardness and slope for Hole 18, Mine A 


\section{Analysis on Field Testing Data}

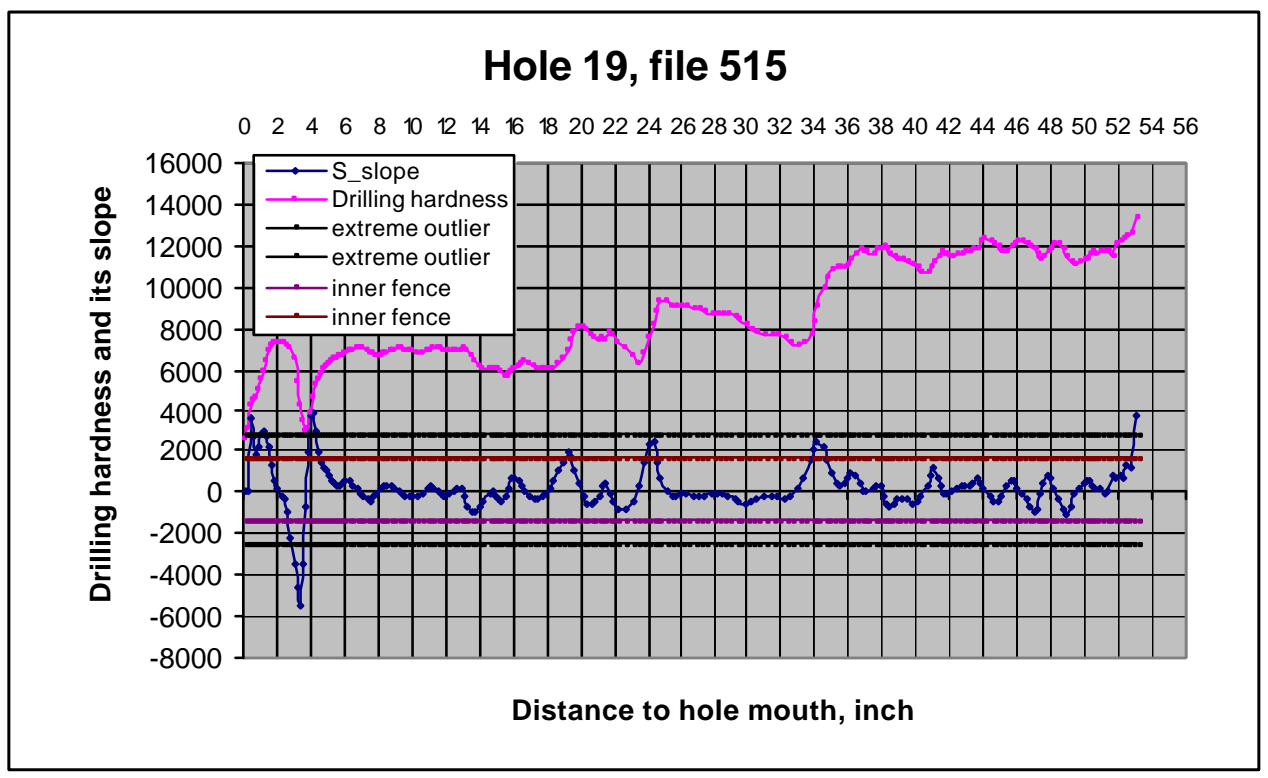

Figure 62 Drilling hardness and slope for Hole 19, Mine A

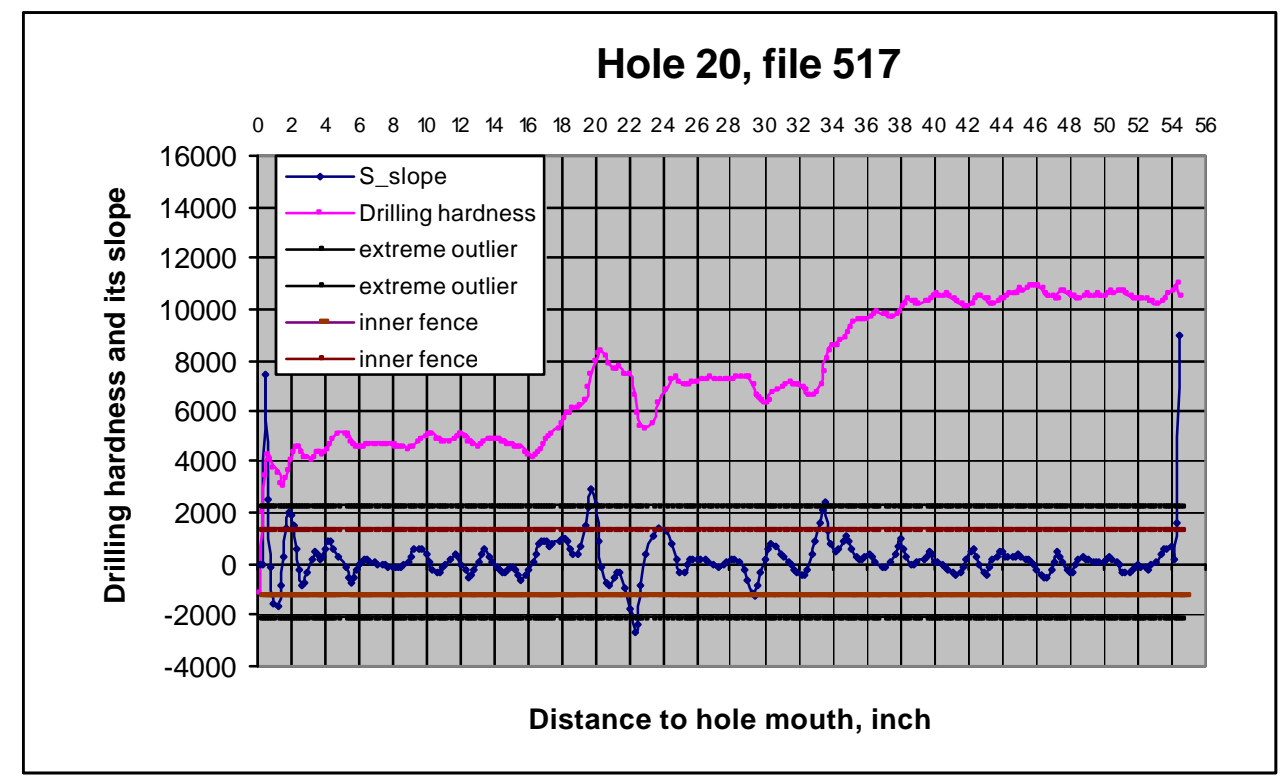

Figure 63 Drilling hardness and slope for Hole 20, Mine A 


\section{Analysis on Field Testing Data}

Table 26 Locations of interfaces and discontinuities determined from the field data using the scope data and the theoretical prediction(inches)

\begin{tabular}{|c|c|c|c|c|c|c|c|c|}
\hline \multirow{2}{*}{$\begin{array}{c}\text { Hole } \\
\text { No. }\end{array}$} & \multicolumn{3}{|c|}{ Interface } & \multicolumn{3}{|c|}{ Discontinuity verified } & \multirow{2}{*}{$\begin{array}{c}\text { Derived but not } \\
\text { observed }\end{array}$} & \multirow{2}{*}{$\begin{array}{c}\text { Observed but } \\
\text { not derived }\end{array}$} \\
\hline & observed & derived & error & observed & derived & error & & \\
\hline 13 & 33.5 & $34.915^{*}$ & 1.415 & $\begin{array}{l}20.5 \\
45.25\end{array}$ & $\begin{array}{l}21.3 \\
46.73\end{array}$ & $\begin{array}{l}0.8 \\
1.48\end{array}$ & $\begin{array}{c}23.93,32.14,41.16 \\
42.91,49.51,51.44\end{array}$ & 36.25 \\
\hline 14 & 34.25 & 34.96 & 0.71 & $\begin{array}{c}36 \\
46.5\end{array}$ & $\begin{array}{l}36.27 \\
46.56\end{array}$ & $\begin{array}{l}0.27 \\
0.06\end{array}$ & $21.79,47.73$ & $40.5,42.7$ \\
\hline 15 & 34 & $35.535 *$ & 1.535 & 47.9 & 44.47 & 3.79 & $37.17,43.43$ & \\
\hline 16 & 36.4 & 35.46 & 0.94 & 44.4 & 42.88 & 1.5 & $\begin{array}{c}20.79,36.79,38.13 \\
46.73\end{array}$ & 5.9 \\
\hline 18 & 33.5 & 35.01 & 1.51 & & & & 20.12 & $34.25,35.25,44.5$ \\
\hline 19 & 36.25 & N/D & $\mathrm{N} / \mathrm{D}$ & & & & & $37,40,42$ \\
\hline 20 & 31.5 & $\begin{array}{c}33.61 \\
20.955 *\end{array}$ & 2.11 & & & & 29.47 & $\begin{array}{c}33.5,37.5,38.25 \\
39.5\end{array}$ \\
\hline Average & 34.2 & 34.915 & 1.37 & & & 1.32 & & \\
\hline
\end{tabular}

Notes: * average location calculated from two interfaces detected within 4 inches

Figures 56 to 63 show the distributions of the drilling hardness and slope along Holes $13,14,15,16,18,19$, and 20 respectively. Table 26 shows the locations of discontinuities determined from the field data using the scope data (observed) and the theoretical prediction (derived) by applying the criteria developed in Section 4.2. The analysis result from Hole 17 was not taken into Table 26 because Hole 17 was not scoped and it would be used in the rock type classification section. It can be seen that the maximum error for the interface between the light gray sandstone and dark gray shale between the observed and derived is 2.11 inches, the minimum is 0.71 inches, and the average error is 1.37 inches. Although drilling hardness indicates the variation around locations 24 and 34 inches, there are no interfaces detected for Hole 19 by applying the basic criteria. One more interface, located at 20.955 inches, was derived from Hole 20. Looking at the distributions of the drilling hardness along 20 (Figure 62), it can be seen that there are three distinct levels in drilling hardness. This indicates that the strength of the first 20.955 inches rock layer is much weaker than that of the rock layer from 20 to 33.61 inches for Hole 20, although the scope data shows that both of the layers are dark gray shale. In addition, the scope image shows that interface is a small area rather than a 


\section{Analysis on Field Testing Data}

planar feature ${ }^{[32]}$. Within this small area, there are either stronger or weaker sub-layer(s). This will inevitably cause more than one location where slope is beyond the outer fences. The core $\log$ shows that the location of the interface between the light gray sandstone and dark gray shale is 34.5 inches, which is very close to the derived location which ranges from 33.61 to 35.535 inches.

In Table 26, Column Discontinuity verified means that the discontinuity shows in both the observed from the scope data and derived from drilling hardness. Table 26 shows that for the verified discontinuity, the maximum error is 3.79 inches, the minimum error is 0.06 inches, and the average error is 1.32 inches. Column Derived but not observed means these locations shown in this column were derived from the drilling hardness but were not observed from the scope. Column Observed but not derived means the locations shown in this column were observed from the scope but were not derived from the drilling hardness. In some cases, the scope data can not tell the strength of the rock around the discontinuity, and sometimes the discontinuity can not be visually detected, although it does exist. However, the drilling hardness can tell the relative strength of the rock around the discontinuity. Thus this discontinuity, whether it is a fracture or a plane of weakness, can be derived and detected if it is weak enough. In other words, if the discontinuity is determined by the drilling hardness and slope, it means that the rock around that discontinuity is weak enough for the drilling hardness to be able to detect and identify. Very often, the accuracy of the scope data really depends on the user's experience and different users of the scope probe may have different judgment in determining the locations of the discontinuities and interfaces. Some discontinuities observed from the scope are not preexisting but generated during the drilling process. All of this will inevitably yield some errors and sometimes these errors may misguide researchers' focus. In addition, the core log (Figure 53) shows that the light gray sandstone is cross-bedded with shale streaks and Mica flakes. Table 26 also shows that only 26.09\% (6 out of 23 discontinuities from the scope) of discontinuities are identified. The core $\log$ (Figure 53) shows that there are two distinct fractures from 18 to 24 inches, which match the detected discontinuities from holes 13, 14, 16, and 18 (Table 26). Since the drilling hardness reflects the relative rock strength and resistance to indentation to the 


\section{Analysis on Field Testing Data}

drill bit under an applied stress, it is not sensitive enough to completely detect all the minor variations while drilling through micro rock structures. In other words, the discontinuities in Column Discontinuity verified are the ones that are weak enough in strength to be detected using the slope of the drilling hardness.

The results from the field test conducted at Mine A show that it is reliable and accurate to determine the locations of interfaces between rock layers with average error 1.37 inches using the drilling hardness and slope. However, the accuracy in determining the locations of discontinuities within one rock layer is not conclusive, because it is hard to compare the derived, observed and core logged locations of discontinuities since some of the results from the scope and core log still show some differences. Nevertheless, the error for the verified discontinuities shown in Table 26 is very small, and the average error is 1.32 inches.

\subsubsection{Rock Type Classification Using Supervised Discriminant Analysis}

According to the geological information obtained from the scope data, the core log, and the theoretical prediction, the entry roof at the Mine A is composed of two rock types from the bottom to the top, dark gray shale and light gray sandstone which is crossbedded with shale streaks and mica flakes. In this analysis of rock type classification, the group data used to determine the group center and the covariance matrix is very important. Based on the optimization in section 4.5, the group data of one rock type can be expressed by the following matrix:

$$
g=\left[\begin{array}{l}
g_{1} \\
g_{2} \\
g_{3} \\
g_{4} \\
g_{5}
\end{array}\right]
$$

where $\mathrm{g}$ - the sub-group of drilling parameters (thrust, penetration rate, torque, rotation rate, 


\section{Analysis on Field Testing Data}

and drilling hardness) extracted from the Hole i, i = 1, 2, 3, 4, 5

The basic steps in classifying rock type are:

(1) Choose holes that produce the best result by comparing with the scope data and core $\log$ to build group data (training data). If there are enough holes, the more holes used to build group data, the more reliable the group data. In this field test, holes 15, 16, 17, 18, 19 are chosen.

(2) Determine the group data. Based on the locations of rock layers in each hole, $g$ of each rock type are extracted from the five holes, thus build up the group data in the form of matrix shown in Eq. 38.

(3) Determine the covariance matrix of a certain rock and mahalanobis distance by using Eqs. 31 and 30.

(4) Calculate the posterior probability using Eq. 29 that is a discriminant analysis non-linear model.

(5) Classify/predict rock type based on posterior probability determined in step 4.

Table 27 shows an example of how to determine rock type based on the calculated posterior probability. The rock type of each data point can be determined by comparing posterior probabilities that belong to the two rock types. For instance, shale has the greatest probability 0.99 at location 17.45 inches, therefore the rock at this point can be classified as shale.

Table 27 An example of rock classification (Hole 17, Mine A)

\begin{tabular}{|c|c|c|c|c|c|c|c|c|}
\hline Thrust & P.R* & Torque & rotation rate & $\begin{array}{c}\text { Drilling } \\
\text { hardness }\end{array}$ & $\begin{array}{c}\text { Position of } \\
\text { the drill bit }\end{array}$ & $\begin{array}{c}\text { Probability of } \\
\text { Shale }\end{array}$ & $\begin{array}{c}\text { Probability of } \\
\text { Sandstone }\end{array}$ & Rock Type \\
\hline 1975.55 & 1.73 & 1155.62 & 608.42 & 5546.95 & 5.12 & 1 & 0 & Shale \\
\hline 2010.18 & 1.73 & 1158.77 & 608.39 & 5670.07 & 5.28 & 1 & 0 & Shale \\
\hline 2044.3 & 1.72 & 1164.29 & 608.37 & 5841.79 & 5.44 & 1 & 0 & Shale \\
\hline 2043.36 & 1.73 & 1169.75 & 608.35 & 5786.24 & 5.6 & 1 & 0 & Shale \\
\hline 2042.44 & 1.74 & 1175.09 & 607 & 5707.47 & 5.76 & 1 & 0 & Shale \\
\hline 2025.13 & 1.74 & 1174.65 & 607 & 5601.25 & 6.08 & 1 & 0 & Shale \\
\hline 2008.18 & 1.74 & 1166.46 & 607 & 5528.78 & 6.25 & 1 & 0 & Shale \\
\hline 1991.57 & 1.75 & 1166.66 & 607 & 5425.28 & 6.41 & 1 & 0 & Shale \\
\hline 2009.41 & 1.75 & 1166.82 & 607 & 5500 & 6.57 & 1 & 0 & Shale \\
\hline
\end{tabular}




\section{Analysis on Field Testing Data}

\begin{tabular}{|c|c|c|c|c|c|c|c|c|}
\hline 2290.65 & 1.68 & 1040.75 & $\mathbf{5 9 4 . 5 4}$ & 7208.54 & 17.45 & 0.99 & 0.01 & Shale \\
\hline$\ldots$ & $\ldots$ & $\ldots$ & $\ldots$ & $\cdots$ & $\ldots$ & $\ldots$ & $\cdots$ & $\ldots$ \\
\hline$\ldots$ & $\ldots$ & $\ldots$ & $\ldots$ & $\ldots$ & $\ldots$ & $\ldots$ & $\ldots$ & $\ldots$ \\
\hline 2448.06 & 1.67 & 1046.4 & 597.29 & 8004.45 & 17.61 & 0.98 & 0.02 & Shale \\
\hline 2620.97 & 1.68 & 1057.6 & 598.67 & 8722.64 & 17.77 & 0.97 & 0.03 & Shale \\
\hline 2756.91 & 1.68 & 1091.2 & 600.02 & 9228.56 & 17.93 & 0.92 & 0.08 & Shale \\
\hline 2823.34 & 1.7 & 1124.31 & 600.02 & 9327.98 & 18.09 & 0.91 & 0.09 & Shale \\
\hline 2803.44 & 1.71 & 1151.28 & 600.02 & 9070.39 & 18.25 & 0.91 & 0.09 & Shale \\
\hline 2750.72 & 1.72 & 1178.26 & 600.02 & 8622.59 & 18.41 & 0.93 & 0.07 & Shale \\
\hline 2664.39 & 1.73 & 1193.96 & 600.02 & 8181.17 & 18.57 & 0.97 & 0.03 & Shale \\
\hline 2595.9 & 1.72 & 1182.84 & 601.46 & 8013.7 & 18.74 & 0.96 & 0.04 & Shale \\
\hline 2401.96 & 1.63 & 1121.44 & 618.8 & 8582.62 & 33.31 & 0.95 & 0.05 & Shale \\
\hline$\ldots$ & $\ldots$ & $\ldots$ & $\ldots$ & $\ldots$ & $\ldots$ & $\ldots$ & $\ldots$ & $\ldots$ \\
\hline$\ldots$ & $\ldots$ & $\ldots$ & $\ldots$ & $\ldots$ & $\ldots$ & $\ldots$ & $\ldots$ & $\ldots$ \\
\hline 2712.26 & 1.63 & 1152.47 & 617.39 & 10086.72 & 33.46 & 0.55 & 0.45 & Shale \\
\hline 2934.34 & 1.63 & 1195.89 & 616 & 10966.36 & 33.76 & 0.22 & 0.78 & Sandstone \\
\hline 3086.58 & 1.64 & 1233.76 & 614.65 & 11434.56 & 33.91 & 0.1 & 0.9 & Sandstone \\
\hline 3196.38 & 1.65 & 1265.72 & 613.98 & 11800.08 & 34.06 & 0.05 & 0.95 & Sandstone \\
\hline 3844.36 & 1.64 & 1358.23 & 613.98 & 14878.1 & 51.36 & 0 & 1 & Sandstone \\
\hline$\ldots$ & $\ldots$ & $\ldots$ & $\ldots$ & $\ldots$ & $\ldots$ & $\ldots$ & $\cdots$ & $\ldots$ \\
\hline$\cdots$ & $\cdots$ & $\ldots$ & $\ldots$ & $\ldots$ & $\ldots$ & $\ldots$ & $\ldots$ & $\ldots$ \\
\hline 3861.68 & 1.64 & 1347.12 & 613.98 & 14898.3 & 51.51 & 0 & 1 & Sandstone \\
\hline 3862.17 & 1.64 & 1341.8 & 613.98 & 14913.86 & 51.66 & 0 & 1 & Sandstone \\
\hline 3844.84 & 1.64 & 1336.55 & 613.98 & 14937.68 & 51.81 & 0 & 1 & Sandstone \\
\hline 3844.36 & 1.63 & 1336.62 & 612.54 & 14961.77 & 51.96 & 0 & 1 & Sandstone \\
\hline 3826.08 & 1.63 & 1342.33 & 611.13 & 14791.72 & 52.1 & 0 & 1 & Sandstone \\
\hline 3791.44 & 1.63 & 1341.95 & 611.17 & 14719.71 & 52.25 & 0 & 1 & Sandstone \\
\hline 3757.29 & 1.62 & 1336.32 & 611.21 & 14657.02 & 52.4 & 0 & 1 & Sandstone \\
\hline 3758.02 & 1.62 & 1336.4 & 611.24 & 14662.32 & 52.55 & 0 & 1 & Sandstone \\
\hline
\end{tabular}

P.R.*: penetration rate.

As mentioned above, Holes 15 to 19 were used to extract data points from the corresponding rock layer in order to build up the group data. Thus, the applied discriminant analysis for these holes is called classification, and for Holes 13, 14 and 20 is called prediction.

Figures 64 to 71 show the distributions of the classified/predicted points along the drilled holes very consistent. In these figures, the vertical line at location 34.5 inches, which is obtained from the core log, indicates the position of the interface between gray shale and gray sandstone. These figures show that the last classified/predicted point (from 


\section{Analysis on Field Testing Data}

the hole bottom to the top) of gray shale locates very close to the interface between the gray shale and gray sandstone with error less than 2 inches.

Table 28 shows the detailed classification/prediction results by using the un-sampled drilling data, including original data points, classified/predicted points, and hit rate for each rock type in each drilled hole. For these drilled holes, the hit rates for the two rock types are very high, ranging from 78.7 to $100 \%$. In addition, Table 28 also shows the classification/prediction results from using the sampled drilling data. It can be seen that using the un-sampled drilling data to do rock type classification/prediction does not make much difference compared with using the sampled drilling data. The only difference is the number of the sampled data points is about half of the un-sampled data, but the hit rate is almost the same. Therefore, the following analyses focus on the results using the un-sampled data.

In an attempt to check reliability and applicability of the discriminant analysis, the hit rates of the classified and predicted rock types, which were picked up from Table 28, were presented in Table 29. For Holes 15 to 19, the hit rates of the classified gray shale and gray sandstone range from 89.71 to $100 \%$ and from 91.6 to $98.37 \%$ respectively. The average hit rates for the two rock types are about $96.25 \%$ and $95.25 \%$ respectively. For Holes 13, 14 and 20 that are used for testing the prediction result, the hit rates of the predicted gray shale and gray sandstone range from 83.33 to $97.24 \%$ and from 78.8 to $95.87 \%$ respectively. The average hit rates for the two rock types are $91.23 \%$ and $87.51 \%$ respectively. Table 29 also shows that the hit rates for both of the rock types obtained from the holes used for prediction is a little lower than that from the holes used for classification. The reason is that these holes with higher average hit rate in classifying rock types are used to build up the group data for each rock type, which are supposed to have higher hit rates. If the hit rate from these holes were very low (less than 50\%), it means the discriminant analysis failed. 


\section{Analysis on Field Testing Data}

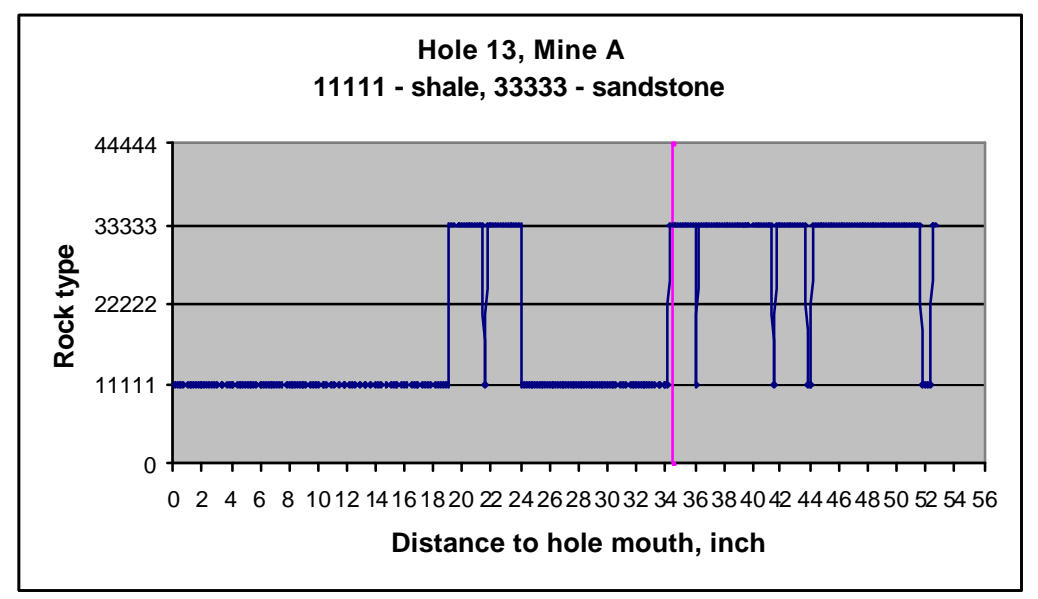

Figure 64 Rock type prediction result, Hole 13

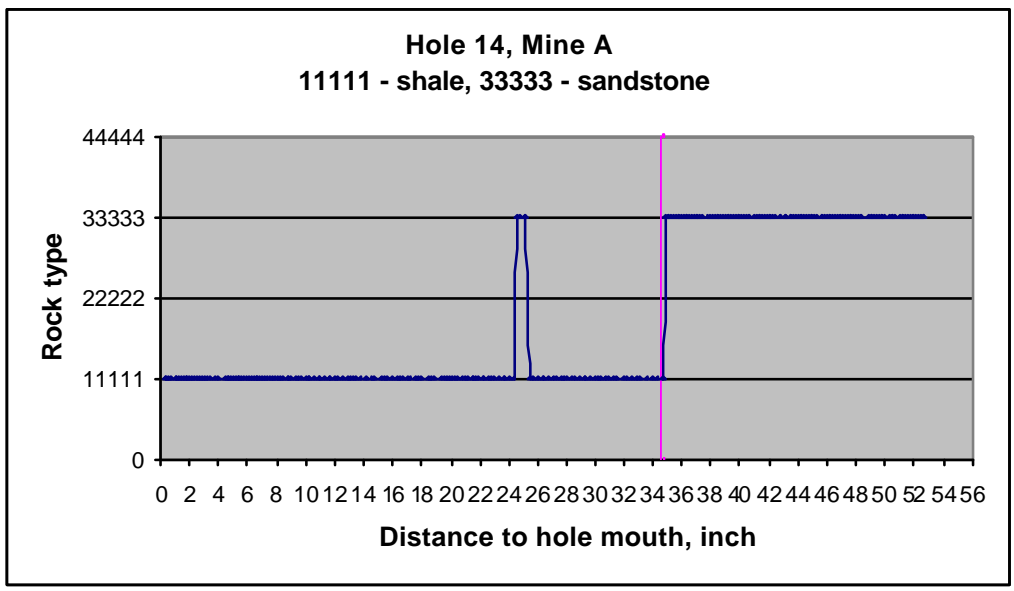

Figure 65 Rock type classification result, Hole 14 


\section{Analysis on Field Testing Data}

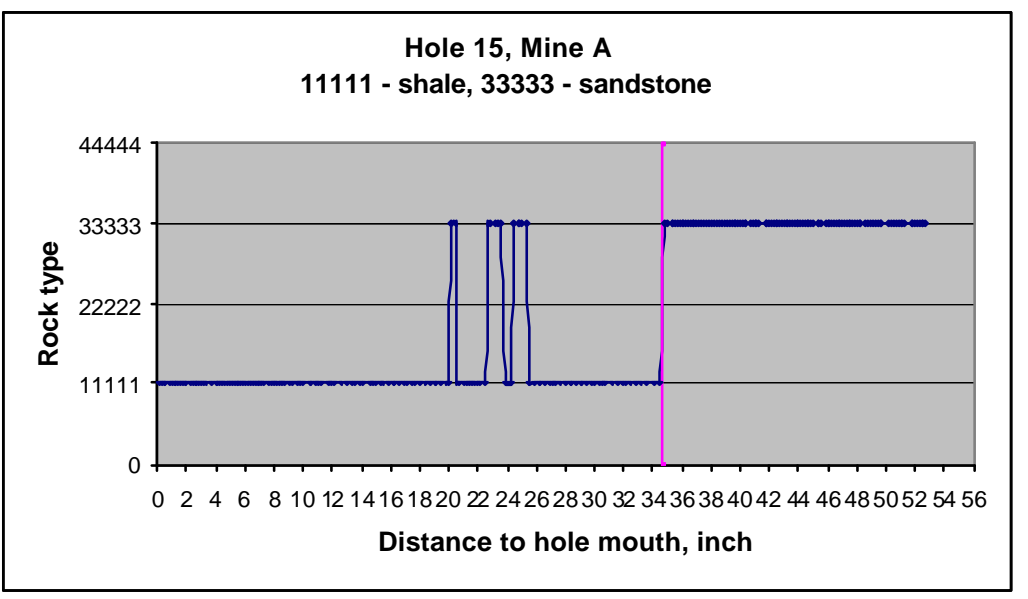

Figure 66 Rock type classification result, Hole 15

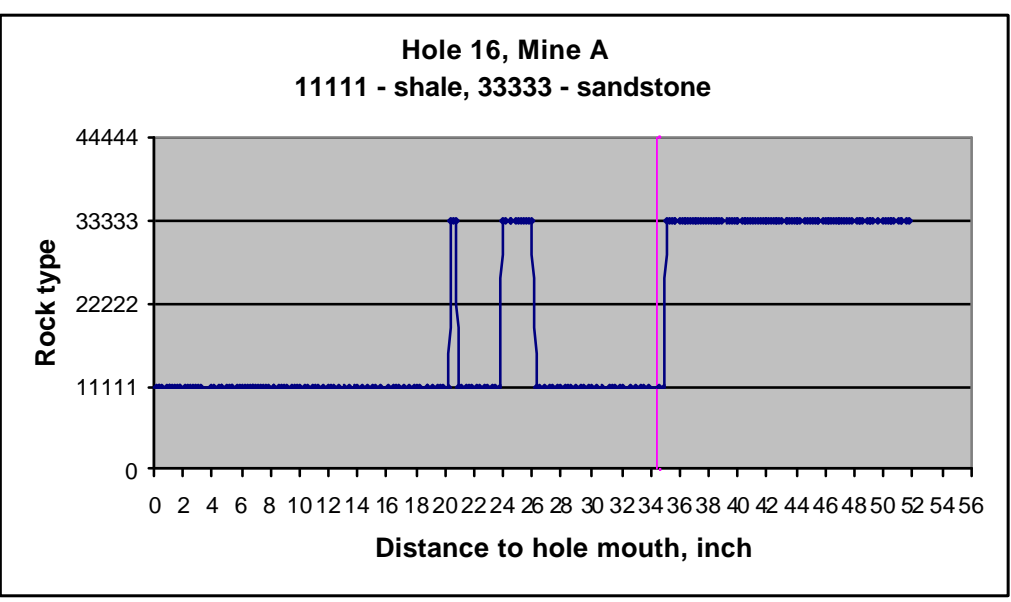

Figure 67 Rock type classification result, Hole 16

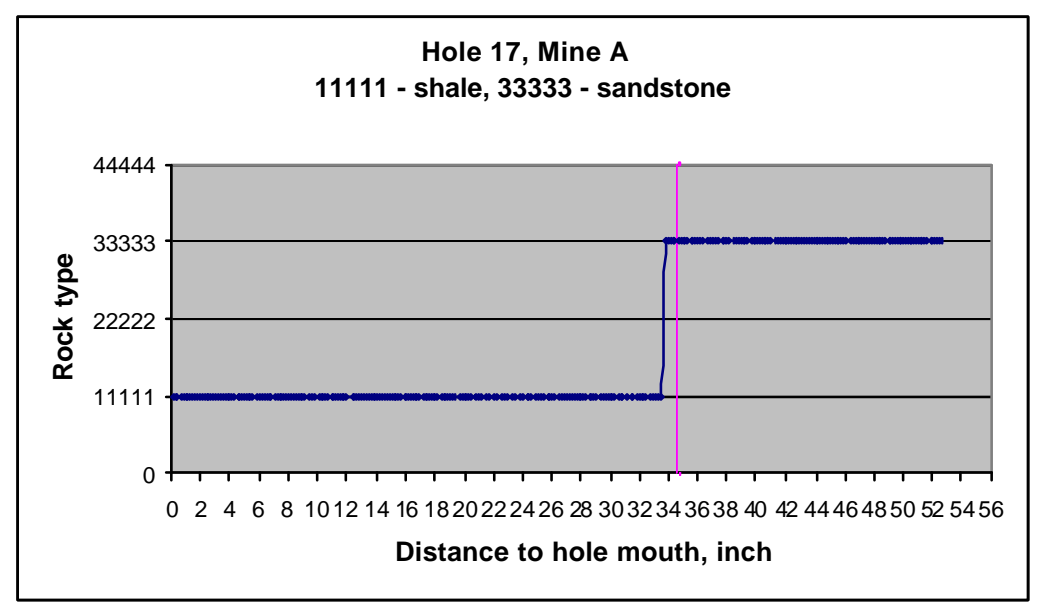

Figure 68 Rock type classification result, Hole 17 


\section{Analysis on Field Testing Data}

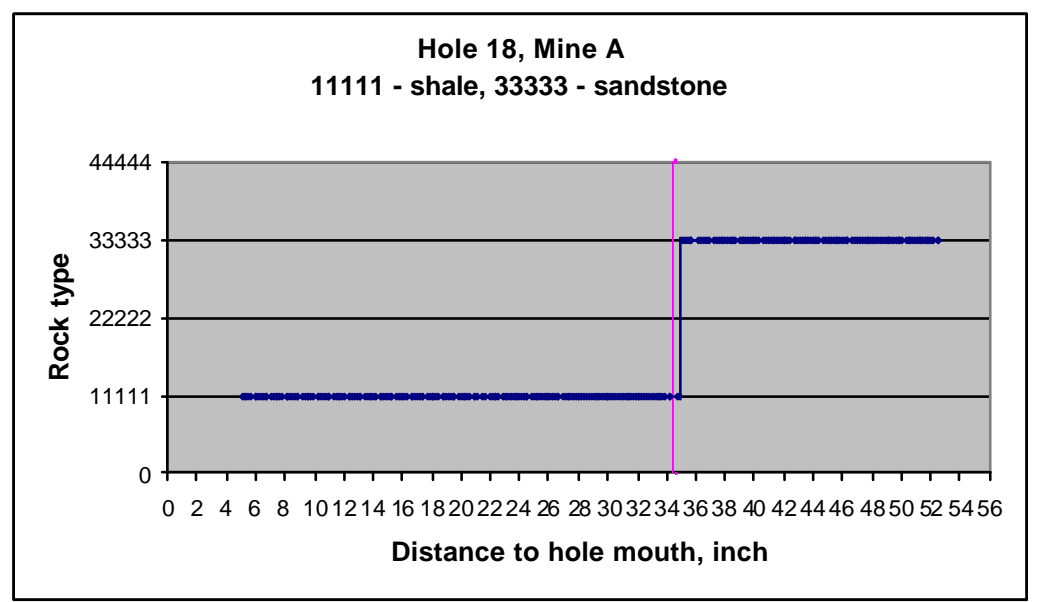

Figure 69 Rock type classification result, Hole 18

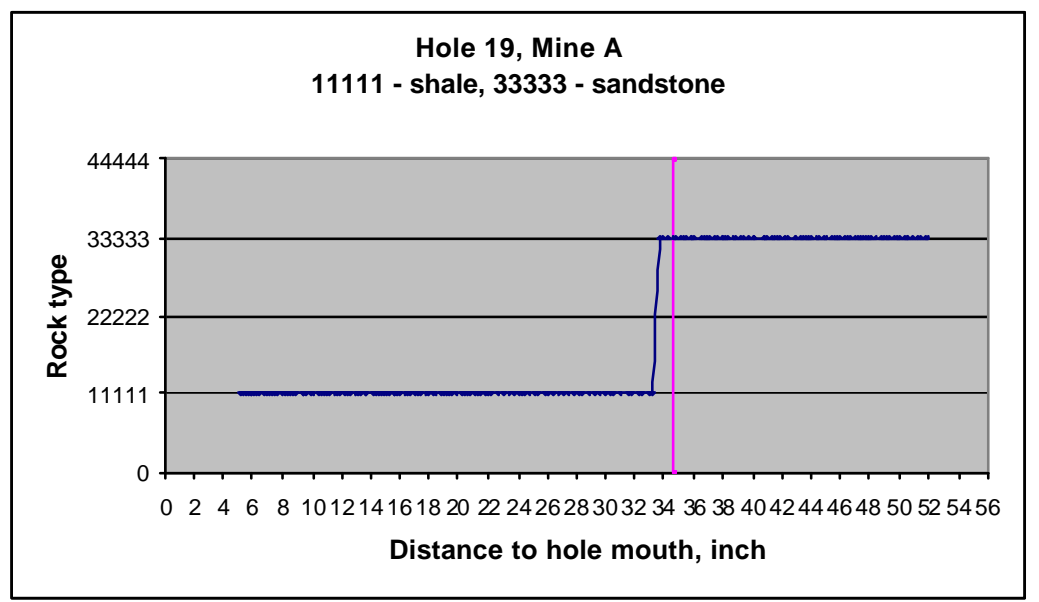

Figure 70 Rock type classification result, Hole 19

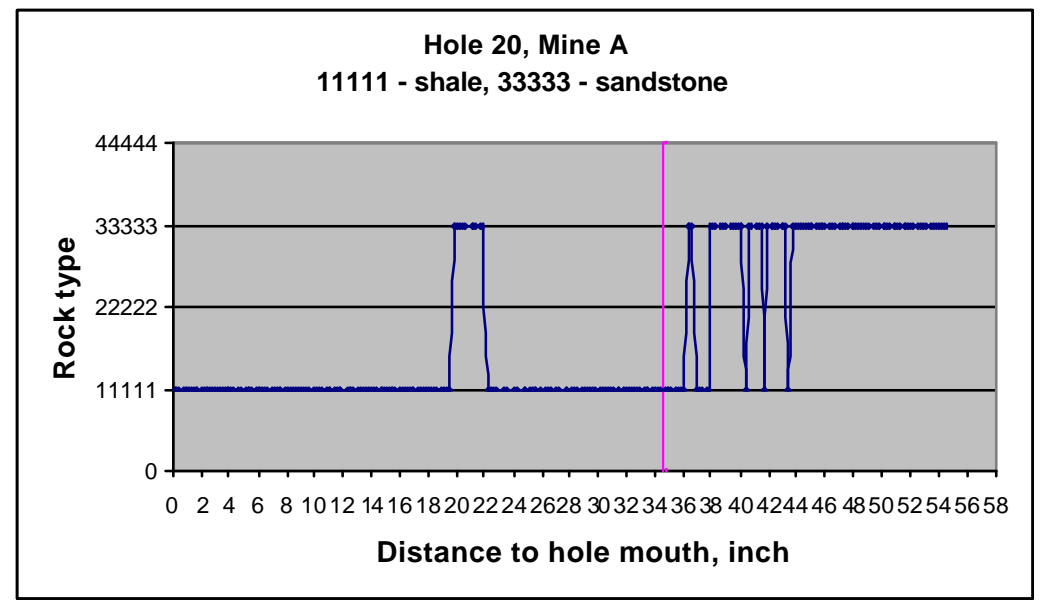

Figure 71 Rock type prediction result, Hole 20 


\section{Analysis on Field Testing Data}

Table 28 Rock type classification/prediction results from Mine A (Holes 13 to 20)

\begin{tabular}{|c|c|c|c|}
\hline \multicolumn{2}{|c|}{ Drilled holes } & Recorded numbers of points & Number of points (un-sampled/sampled) \\
\hline \multirow{6}{*}{ Hole 13} & \multirow{3}{*}{ Gray shale } & Number of original points & $192 / 96$ \\
\hline & & Number of predicted points & $160 / 80$ \\
\hline & & Hit rate $(\%)$ & 83.33/83.33 \\
\hline & \multirow{3}{*}{ Light sandstone } & Number of original points & $133 / 67$ \\
\hline & & Number of predictedpoints & $117 / 59$ \\
\hline & & Hit rate $(\%)$ & $87.97 / 88.1$ \\
\hline \multirow{6}{*}{ Hole 14} & \multirow{3}{*}{ Gray shale } & Number of original points & $145 / 73$ \\
\hline & & Number of predicted points & $141 / 71$ \\
\hline & & Hit rate $(\%)$ & $97.24 / 97.26$ \\
\hline & \multirow{3}{*}{ Light sandstone } & Number of original points & $121 / 61$ \\
\hline & & Number of predicted points & $116 / 58$ \\
\hline & & Hit rate $(\%)$ & 95.87/95.08 \\
\hline \multirow{6}{*}{ Hole 15} & \multirow{3}{*}{ Gray shale } & Number of original points & $142 / 71$ \\
\hline & & Number of classified points & $130 / 65$ \\
\hline & & Hit rate (\%) & $91.55 / 91.55$ \\
\hline & \multirow{3}{*}{ Light sandstone } & Number of original points & $114 / 57$ \\
\hline & & Number of classified points & $110 / 55$ \\
\hline & & Hit rate $(\%)$ & $96.49 / 96.49$ \\
\hline \multirow{6}{*}{ Hole 16} & \multirow{3}{*}{ Gray shale } & Number of original points & $136 / 68$ \\
\hline & & Number of classified points & $122 / 61$ \\
\hline & & Hit rate $(\%)$ & $89.71 / 89.71$ \\
\hline & \multirow{3}{*}{ Light sandstone } & Number of original points & $105 / 53$ \\
\hline & & Number of classified points & $99 / 49$ \\
\hline & & Hit rate $(\%)$ & $94.29 / 92.45$ \\
\hline \multirow{6}{*}{ Hole 17} & \multirow{3}{*}{ Gray shale } & Number of original points & $184 / 92$ \\
\hline & & Number of classified points & $184 / 92$ \\
\hline & & Hit rate $(\%)$ & $100 / 100$ \\
\hline & \multirow{3}{*}{ Light sandstone } & Number of original points & $119 / 60$ \\
\hline & & Number of classified points & $116 / 58$ \\
\hline & & Hit rate $(\%)$ & 97.48/96.67 \\
\hline \multirow{6}{*}{ Hole 18} & \multirow{3}{*}{ Gray shale } & Number of original points & $174 / 87$ \\
\hline & & Number of classified points & $174 / 87$ \\
\hline & & Hit rate $(\%)$ & $100 / 100$ \\
\hline & \multirow{3}{*}{ Light sandstone } & Number of original points & $119 / 60$ \\
\hline & & Number of classified points & $109 / 54$ \\
\hline & & Hit rate $(\%)$ & 91.6/90.0 \\
\hline \multirow[t]{3}{*}{ Hole 19} & \multirow{3}{*}{ Gray shale } & Number of original points & $110 / 55$ \\
\hline & & Number of classified points & $110 / 55$ \\
\hline & & Hit rate $(\%)$ & $100 / 100$ \\
\hline
\end{tabular}




\section{Analysis on Field Testing Data}

\begin{tabular}{|l|c|c|c|}
\hline \multirow{4}{*}{ Light sandstone } & Number of original points & $79 / 40$ \\
\cline { 3 - 4 } & & Number of classified points & $78 / 39$ \\
\cline { 3 - 4 } & & Hit rate (\%) & $\mathbf{9 8 . 7 3 / 9 7 . 5}$ \\
\hline \multirow{3}{*}{ Hole 20 } & Gray shale & Number of original points & $160 / 80$ \\
\cline { 3 - 4 } & & Number of predicted points & $149 / 74$ \\
\cline { 3 - 4 } & \multirow{3}{*}{ Light sandstone } & Hit rate (\%) & $\mathbf{9 3 . 1 3 / 9 2 . 5}$ \\
\cline { 3 - 4 } & & Number of original points & $108 / 54$ \\
\cline { 3 - 4 } & & Hit rate (\%) & $85 / 42$ \\
\cline { 3 - 4 } & & & $\mathbf{7 8 . 7 0 / 7 7 . 7 8}$ \\
\hline
\end{tabular}

Table 29 Hit rates of the predicted and classified rock types

\begin{tabular}{|c|c|c|}
\hline Predicted rock type & Gray shale (\%) & Light sandstone (\%) \\
\hline hole 13 & 83.33 & 87.97 \\
\hline hole 14 & 97.24 & 95.87 \\
\hline hole 20 & 93.13 & 78.70 \\
\hline Average & $\mathbf{9 1 . 2 3}$ & $\mathbf{8 7 . 5 1}$ \\
\hline Classified rock type & Gray shale & Light sandstone \\
\hline hole 15 & 91.55 & 96.49 \\
\hline hole 16 & 89.71 & 94.29 \\
\hline hole 17 & 100 & 97.48 \\
\hline hole 18 & 100 & 91.6 \\
\hline hole 19 & 100 & 98.37 \\
\hline Average & $\mathbf{9 6 . 2 5}$ & $\mathbf{9 5 . 6 5}$ \\
\hline
\end{tabular}

\subsection{Test Conducted at Mine B}

\subsubsection{Test Site and Testing Parameters}

The test site was selected at areas near an intersection close to the exposed sandstone intruder. Four testing areas were chosen around the intersection as shown in Figure 72. The arrangement of drilling holes at each area is also shown in Figure 72. In addition, core logs were obtained by drilling boreholes in these four areas (Figure 72). All these areas allow one core log available except Area-2 which has two. The core logs for Area1, Area-2, Area-3 and Area-4 are shown in Figures 73, 74, and 75, respectively. 


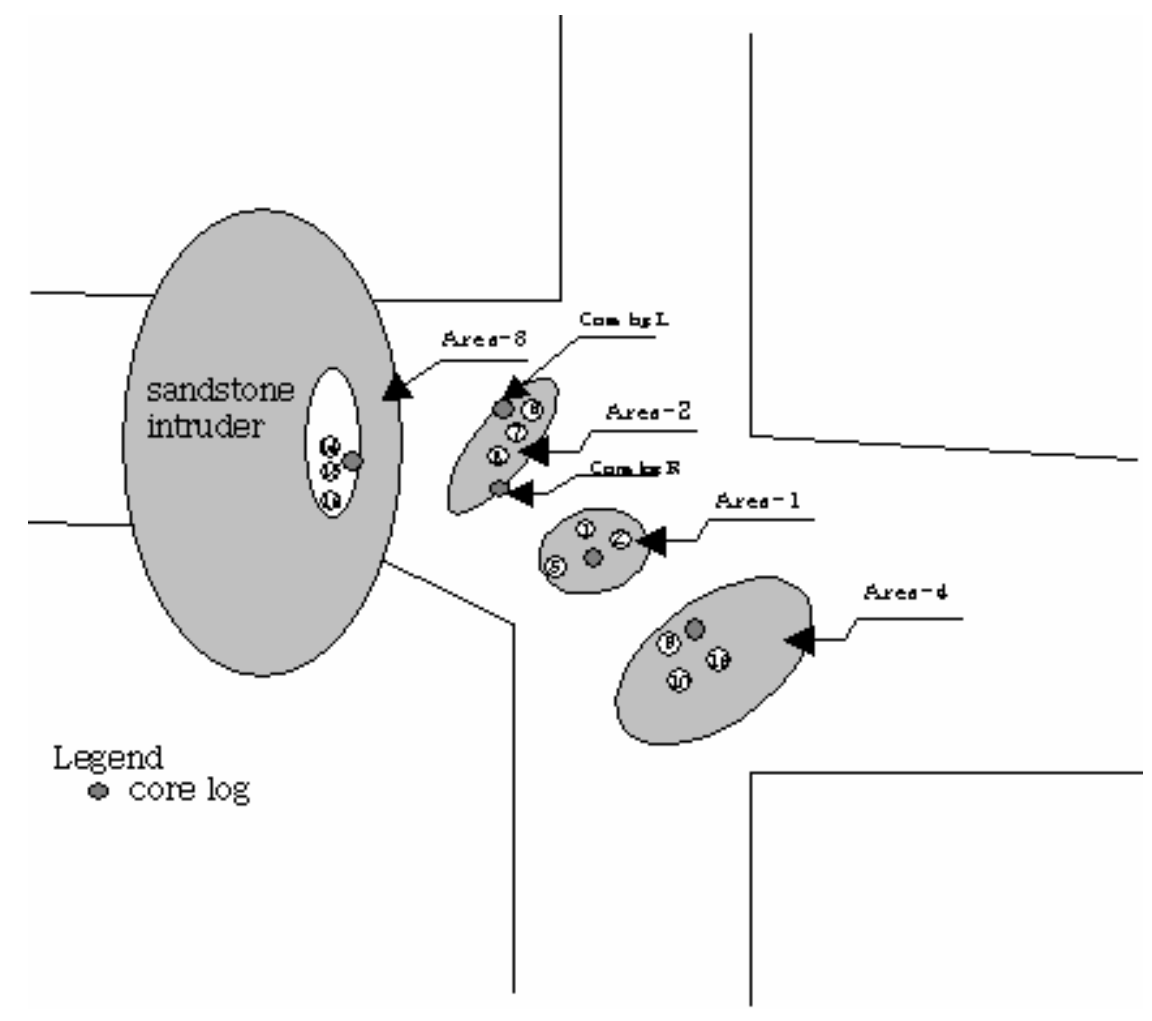

Figure 72 Testing sites at Mine B

Table 30 shows the testing plan and controlling parameters in each Area. All these holes were drilled by using 1-3/8-in bit. Three holes in each Area are picked to be analyzed and compared with the core log in each corresponding Area.

Table 30 Testing parameters at Mine B

\begin{tabular}{|c|c|c|c|c|c|c|}
\hline Hole No. & $\begin{array}{c}\text { Bit size } \\
\text { inch }\end{array}$ & $\begin{array}{c}\text { P.R } \\
\text { in/sec }\end{array}$ & $\begin{array}{c}\text { rotation rate } \\
\text { Ro./min. }\end{array}$ & $\begin{array}{c}\text { Thrust cap } \\
\text { psi }\end{array}$ & $\begin{array}{c}\text { Hole Length } \\
\text { in }\end{array}$ & File Name \\
\hline Hole 1, Area -1 & $1-3 / 8$-in & 0.8 & Free & 650 & 54 & File 588 \\
\hline Hole 2, Area -1 & $1-3 / 8$-in & 0.8 & Free & 650 & 53 & File 589 \\
\hline Hole 5, Area -1 & $1-3 / 8$-in & 1.1 & Free & 650 & 53 & File 592 \\
\hline Hole 6, Area -2 & $1-3 / 8$-in & 1.1 & Free & 650 & 54 & File 593 \\
\hline Hole 7, Area -2 & $1-3 / 8$-in & 1.1 & Free & 800 & 54 & File 594 \\
\hline Hole 8, Area -2 & $1-3 / 8$-in & 1.5 & Free & 650 & 54 & File 595 \\
\hline Hole 14, Area -3 & $1-3 / 8$-in & Free & 500 & 650 & 54 & File 602 \\
\hline Hole 15, Area -3 & $1-3 / 8$-in & Free & 400 & 650 & 54 & File 603 \\
\hline Hole 16, Area -3 & $1-3 / 8$-in & Free & 400 & 650 & 54 & File 604 \\
\hline
\end{tabular}




\section{Analysis on Field Testing Data}

\begin{tabular}{|c|c|c|c|c|c|c|}
\hline Hole 9, Area -4 & $1-3 / 8$-in & 1.1 & Free & 650 & 54 & File 638 \\
\hline Hole 10, Area -4 & $1-3 / 8$-in & 1.1 & Free & 650 & 54 & File 639 \\
\hline Hole 16, Area -4 & $1-3 / 8$-in & 1.1 & Free & 800 & 54.5 & File 645 \\
\hline
\end{tabular}
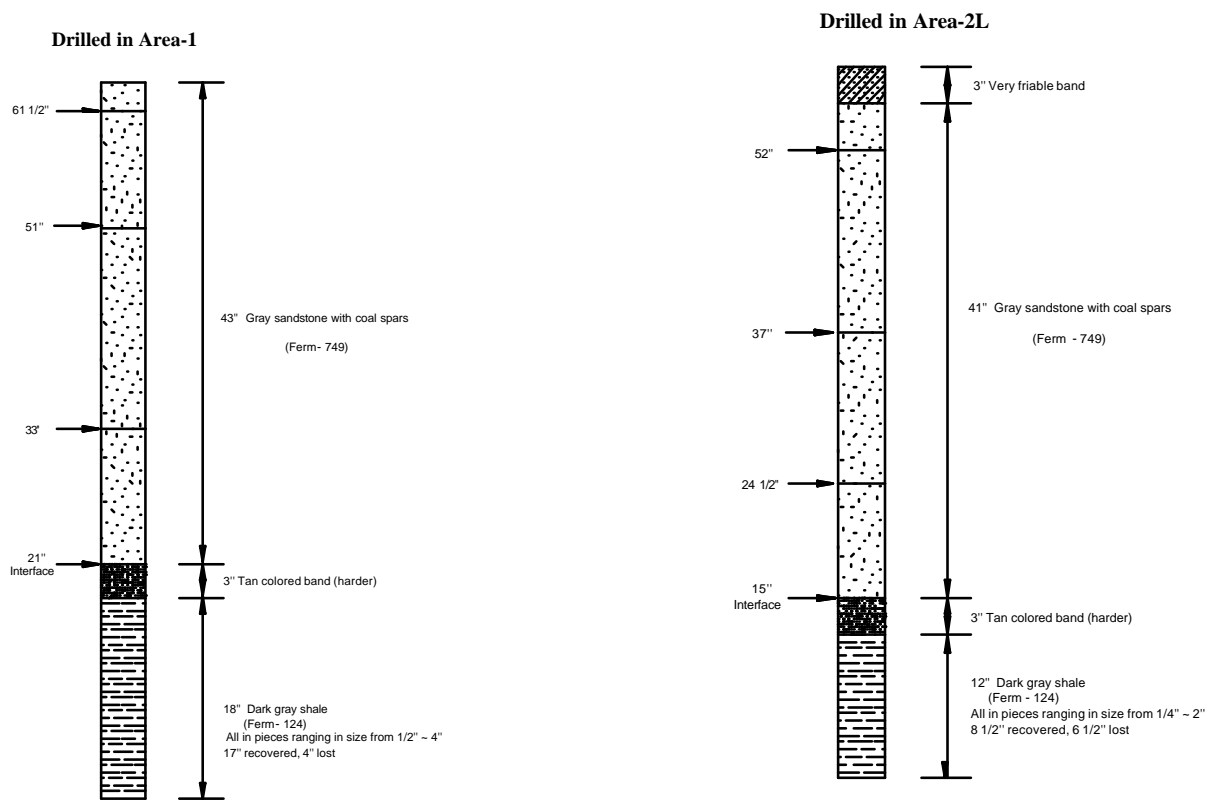

Figure 73 Core log in Area-1 and Area-2L 


\section{Analysis on Field Testing Data}
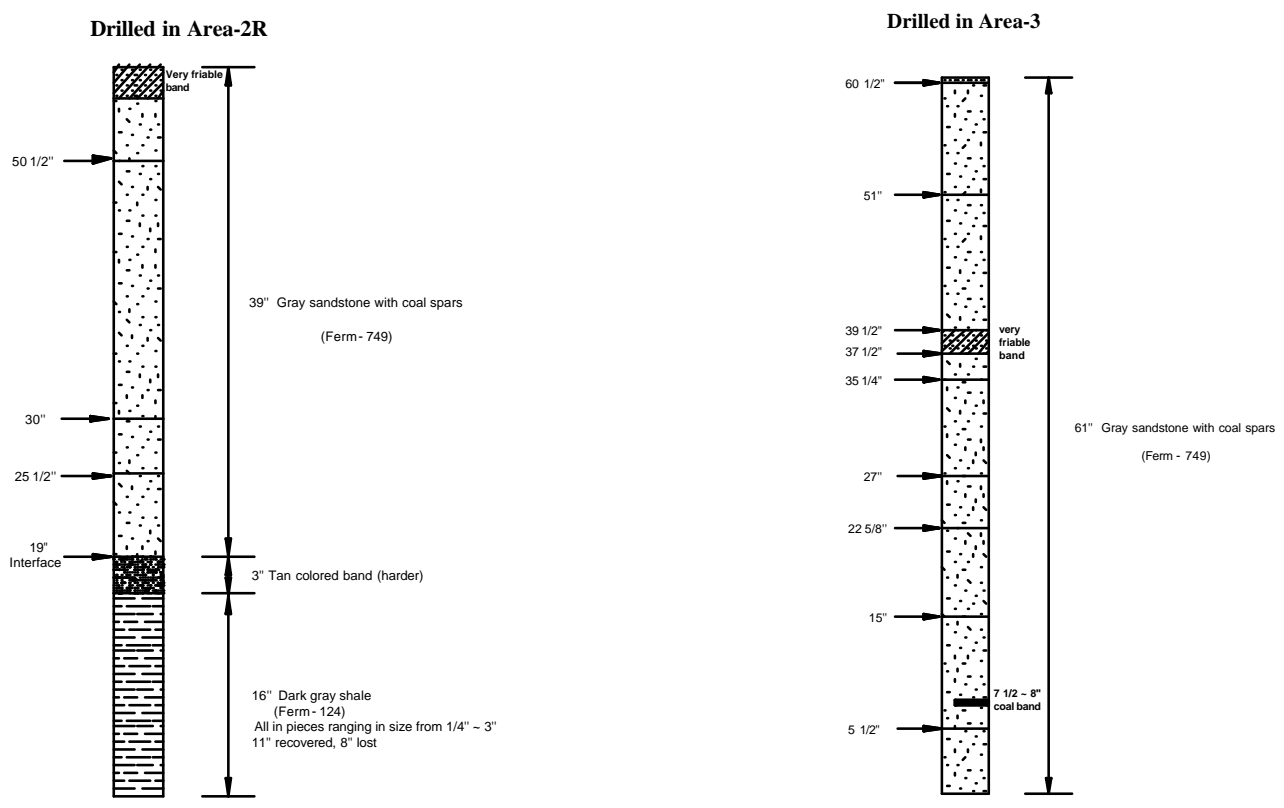

Figure 74 Core log in Area-2R and Area-3

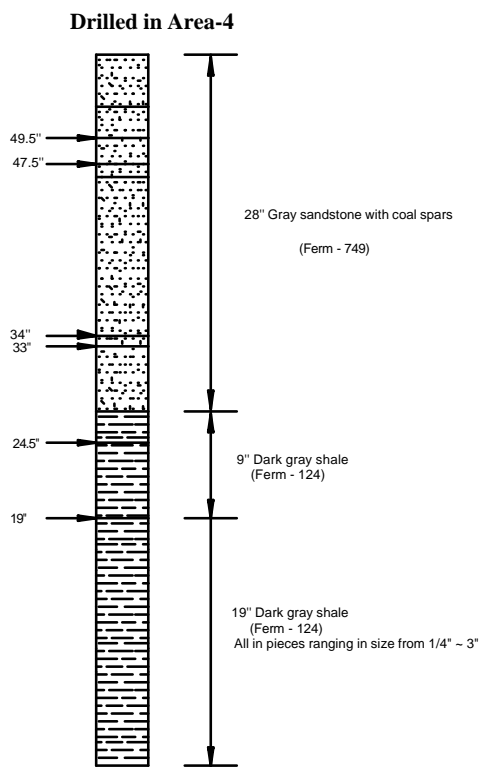

Figure 75 Core log in Area-4 


\section{Analysis on Field Testing Data}

\subsubsection{Process Results from Mine B}

Applying the same procedure used in the laboratory drilling test and field test conducted at Mine A, the field drilling data were used to calculate the drilling hardness and slope. The results from the theoretical prediction and core logs are shown in Table 31. Empty cells in Table 31 indicate unavailable data. The calculated drilling hardness and slope of each hole are presented in Appendix IV (Figures IV-1 to IV-12). While determining the locations of interfaces, only two holes, Hole 1 in Area-1 and Hole 7 in Area- 2 could not detect the interfaces by applying the criteria developed in Section 4.2.

Table 31 Locations of interfaces and discontinuities determined from the field data using the core $\log$ and the theoretical prediction (inches)

\begin{tabular}{|c|c|c|c|c|c|c|c|c|}
\hline \multirow{2}{*}{$\begin{array}{l}\text { Hole } \\
\text { No. }\end{array}$} & \multicolumn{3}{|c|}{ Interface } & \multicolumn{3}{|c|}{ Discontinuity verified } & \multirow{2}{*}{$\begin{array}{c}\text { Derived but not } \\
\text { core logged }\end{array}$} & \multirow{2}{*}{$\begin{array}{c}\text { Core logged } \\
\text { but not derived }\end{array}$} \\
\hline & Core log & derived & error & Core log & derived & error & & \\
\hline $\begin{array}{l}\text { Hole 1, } \\
\text { Area-1 }\end{array}$ & 21 & N/A* & N/A & N/A & N/A & N/A & N/A & 33,51 \\
\hline $\begin{array}{l}\text { Hole 2, } \\
\text { Area-1 }\end{array}$ & 21 & $\begin{array}{l}22.04 \\
26.19\end{array}$ & $\begin{array}{l}2.98 \\
\text { N/A }\end{array}$ & N/A & N/A & N/A & $25.16,44.06$ & 33,51 \\
\hline $\begin{array}{l}\text { Hole 5, } \\
\text { Area-1 }\end{array}$ & 21 & 18.37 & 2.63 & 51 & 49.23 & 1.77 & $18.58,23.06$ & 33 \\
\hline $\begin{array}{c}\text { Average } \\
\text { Area1 }\end{array}$ & 21 & 20.205 & 0.795 & N/A & N/A & 1.77 & N/A & N/A \\
\hline $\begin{array}{l}\text { Hole 6, } \\
\text { Area-2 }\end{array}$ & 15 & 17.2 & 2.2 & 24.5 & 25.07 & 0.57 & N/A & $12,37,52$ \\
\hline $\begin{array}{l}\text { Hole 7, } \\
\text { Area-2 }\end{array}$ & 15 & N/A & N/A & 52 & 50.33 & 1.67 & 46.14 & 12,37 \\
\hline $\begin{array}{l}\text { Hole 8, } \\
\text { Area-2 }\end{array}$ & 15 & 9.72 & 5.28 & $\begin{array}{l}12 \\
52\end{array}$ & $\begin{array}{l}11.96 \\
49.43\end{array}$ & $\begin{array}{l}0.04 \\
2.57\end{array}$ & N/A & $24.5,37$ \\
\hline $\begin{array}{l}\text { Average } \\
\text { Area2 }\end{array}$ & 15 & 13.46 & 1.54 & N/A & N/A & 1.2125 & N/A & N/A \\
\hline $\begin{array}{c}\text { Hole 14, } \\
\text { Area-3 }\end{array}$ & $\begin{array}{c}\text { No } \\
\text { interface }\end{array}$ & $\begin{array}{c}\text { No } \\
\text { interface }\end{array}$ & 0 & $\begin{array}{c}8 \\
15\end{array}$ & $\begin{array}{l}9.84 \\
13.63\end{array}$ & $\begin{array}{l}1.84 \\
1.37\end{array}$ & N/A & $\begin{array}{c}5.5,22.63,27 \\
35.25,37.5,39.5 \\
51\end{array}$ \\
\hline $\begin{array}{c}\text { Hole 15, } \\
\text { Area-3 }\end{array}$ & $\begin{array}{c}\text { No } \\
\text { interface }\end{array}$ & $\begin{array}{c}\text { No } \\
\text { interface }\end{array}$ & 0 & $\begin{array}{c}15 \\
35.25\end{array}$ & $\begin{array}{l}13.73 \\
35.9\end{array}$ & $\begin{array}{l}1.27 \\
0.65\end{array}$ & N/A & $\begin{array}{c}5.5,8,22.63,27 \\
37.5,39.5,51\end{array}$ \\
\hline $\begin{array}{c}\text { Hole 16, } \\
\text { Area-3 }\end{array}$ & $\begin{array}{c}\text { No } \\
\text { interface }\end{array}$ & $\begin{array}{c}\text { No } \\
\text { interface }\end{array}$ & 0 & 8 & 9.93 & 1.93 & N/A & $\begin{array}{c}15,22.63,27, \\
35.25,37.5,39.5 \\
51\end{array}$ \\
\hline Average & $\mathbf{0}$ & $\mathbf{0}$ & $\mathbf{0}$ & N/A & N/A & 1.412 & N/A & \\
\hline
\end{tabular}




\section{Analysis on Field Testing Data}

\begin{tabular}{|c|c|c|c|c|c|c|c|c|}
\hline Area3 & & & & & & & & \\
\hline $\begin{array}{l}\text { Hole 9, } \\
\text { Area-4 }\end{array}$ & 28 & $29.33^{* * *}$ & 1.33 & $\begin{array}{c}19 \\
49.5\end{array}$ & $\begin{array}{l}21.98 \\
50.13\end{array}$ & $\begin{array}{l}2.98 \\
0.63\end{array}$ & $7.11,51.14$ & $33,34,47.5$ \\
\hline $\begin{array}{l}\text { Hole 10, } \\
\text { Area-4 }\end{array}$ & 28 & 30.54 & 2.54 & 24.5 & 22.89 & 1.61 & 7.42 & $\begin{array}{c}19,33,34,47.5 \\
49.5\end{array}$ \\
\hline $\begin{array}{c}\text { Hole 16, } \\
\text { Area-4 }\end{array}$ & 28 & $\begin{array}{c}31.755 * * \\
7.15\end{array}$ & 3.755 & $\begin{array}{c}19 \\
49.5\end{array}$ & $\begin{array}{l}18.21 \\
50.71\end{array}$ & $\begin{array}{l}1.21 \\
1.21\end{array}$ & N/A & $24.5,33,34,47.5$ \\
\hline $\begin{array}{l}\text { Average } \\
\text { Area44 }\end{array}$ & 28 & 30.542 & 2.542 & N/A & $\mathrm{N} / \mathrm{A}$ & 1.528 & N/A & N/A \\
\hline $\begin{array}{c}\text { Total- } \\
\text { Average }\end{array}$ & & & 1.22 & N/A & N/A & 1.481 & $\bar{N} / \mathrm{A}$ & N/A \\
\hline
\end{tabular}

Notes: * - not available, ** - average location calculated from two interfaces detected within 4 inches

The core $\log$ s obtained from the four areas show that the rock properties vary significantly from Area-4 $\rightarrow$ Area-1 $\rightarrow$ Area-2 $\rightarrow$ Area-3 (Figures 73, 74, and 75). The number of rock layers over the roof gradually change from two (shale and sandstone) in Area-4 to one rock layer (sandstone) in Area-3. In the Area-2, the location of the interface between the shale and sandstone obtained from the core log Area-2L (Figure 74 ) is 4 inches difference from the one obtained from the core log Area-2R (Figure 73). Therefore, the theoretical prediction results from holes 6, 7 and 8 are solely compared with the core $\log$ Area-2L since these holes are closes to that core-hole (Figure 72).

It can be seen from Table 31 that the maximum, the minimum, and the average errors in identifying the location of the interface between the shale and sandstone in the four areas are 5.28, 0, and 1.22 inches respectively. Although an interface at location 26.19 inches for Hole 2, 4.15 inches away from another interface that locates at 22.04 inches, was detected, it is actually located in the transition zone from shale to sandstone (Figure IV-2). During this transition zone, this detected interface may indicate that there is a sublayer that is stronger than adjacent rock. Another interface at 7.15 inches was also detected from Hole 16, Area-4. If looking at the distribution of drilling hardness in Figure IV-12, Appendix IV, it can be found that the drilling hardness below 7.15 inches is much lower than upper rock layers. This is the reason why this interface at 7.15 inches was detected. The core log obtained from Area-2L shows that there is a 3 inch thick Tan colored band (harder) but does not indicate its composition This stronger and harder band can roughly be reflected by the drilling hardness in Figures IV-5 for Hole 7 and IV- 


\section{Analysis on Field Testing Data}

6 for Hole 8 (Appendix IV). It can bee seen that the drilling hardness from 9 to 12 inches is larger than that from adjacent points. Figure 76 shows the comparison result of the interface location, which is obtained by averaging three holes in each area, between the core logged and the theoretically derived. It shows that the derived location of the interface basically reflects the variation in roof geology, i.e., from Area- $4 \rightarrow$ Area- $1 \rightarrow$ Area- $2 \rightarrow$ Area-3, the shale gradually becomes thinner and thinner until the roof turns into sandstone.

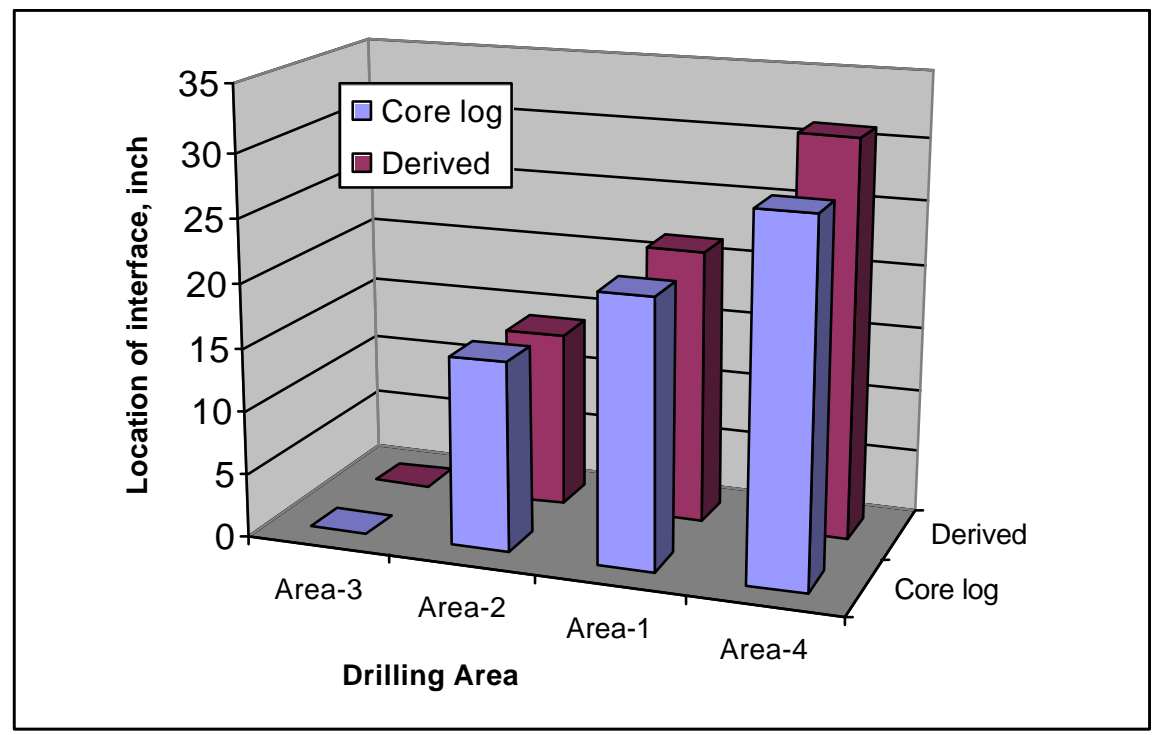

Figure 76 Comparison of the interface location in different areas

Table 31 also shows that in the four areas only $25.86 \%$ discontinuities are identified with the maximum, minimum and average errors 2.98, 0.04, and 1.481 inches respectively. As explained in Section 5.1.2, the discontinuities in Column Discontinuity verified can be considered as the ones that are weak enough in strength to be detected using the slope of the drilling hardness.

Totally, 19 holes were analyzed from Mine A and Mine B. The number of interfaces detected from the scope camera and core log are 19 and the number of interfaces derived 


\section{Analysis on Field Testing Data}

from the drilling hardness is 22 . For the interfaces obtained from the scope camera and core $\log , 16$ out of 19 were verified.

\subsubsection{Rock Type Classification Results}

According to the geological information obtained from the core logs and the theoretical prediction, the entry roof at the Mine B consists of two rock layers from the bottom to the top, gray shale and gray sandstone. Rock layers over the roof gradually change from two (shale and sandstone) in Area-4 to one rock layer (sandstone) in Area-3 (Figure 76). In an attempt to compare the influence of different group data on the analysis results of rock type classification, the group data for rocks gray shale and gray sandstone were built up with two ways. First, the group data for each rock type were built up by solely using the holes drilled in Area-4 (holes 9, 10 and 16), and secondly the group data were built up by picking holes drilled in the four areas (holes 5 (Area-1), 8 (Area-2), 15 (Area-3), 10 (Area-4), and 16 (Area-4)).

Figures IV-13 to IV-36 (Appendix IV) show the distributions of the classified/predicted points along the drilled holes. Figures IV-13 to IV-24 are the results classified/predicted using the group data built from the holes drilled in Area-4 and Figures IV-25 to IV-36 are the results classified/predicted using the group data built from the holes picked from the four areas. In these figures, the vertical line, which varies with different drilling areas, indicates the core logged position of the interface between gray shale and gray sandstone.

Table IV-1 (Appendix IV) shows the detailed classification/prediction results, including original data points, classified/predicted points, and hit rate for each rock type in each drilled hole. 


\section{Analysis on Field Testing Data}

Table 32 Hit rates of the predicted and classified rock types (Group data obtained from Area-4)

\begin{tabular}{|c|c|c|}
\hline Predicted rock type & Gray shale (\%) & Gray sandstone (\%) \\
\hline Hole 1, Area-1 & 100 & 9.32 \\
\hline Hole 2, Area-1 & 100 & 38.76 \\
\hline Hole 5, Area-1 & 100 & 75.4 \\
\hline Average, Area-1 & $\mathbf{1 0 0}$ & $\mathbf{4 1 . 1 6}$ \\
\hline Hole 6, Area-2 & 100 & 0 \\
\hline Hole 7, Area-2 & 100 & 79.09 \\
\hline Hole 8, Area-2 & 100 & 84.16 \\
\hline Average, Area-2 & $\mathbf{1 0 0}$ & $\mathbf{5 4 . 4 2}$ \\
\hline Hole 14, Area-3 & N/A & 0 \\
\hline Hole 15, Area-3 & N/A & 0 \\
\hline Hole 16, Area-3 & N/A & $\mathbf{0}$ \\
\hline Average, Area-3 & N/A & 85.35 \\
\hline Classified rock type & Gray shale & Gray sandstone \\
\hline Hole 9, Area-4 & 97.93 & 81.19 \\
\hline Hole 10, Area-4 & 100 & $\mathbf{8 5 . 9 2}$ \\
\hline Hole 16, Area-4 & 100 & $\mathbf{9 9 . 3 1}$ \\
\hline Average, Area - 4 & & \\
\hline
\end{tabular}

Table 33 Hit rates of the predicted and classified rock types (Group data obtained from Area-1, 2, 3 and 4)

\begin{tabular}{|c|c|c|}
\hline $\begin{array}{c}\text { Predicted/classified } \\
\text { rock type }\end{array}$ & Gray shale $\mathbf{( \% )}$ & Gray sandstone $\mathbf{\%})$ \\
\hline Hole 1, Area-1 & 100 & 78.81 \\
\hline Hole 2, Area-1 & 98.46 & 85.27 \\
\hline *Hole 5, Area-1 & 85.48 & 100 \\
\hline Average, Area-1 & $\mathbf{9 4 . 6 5}$ & $\mathbf{8 8 . 0 3}$ \\
\hline Hole 6, Area-2 & 87.5 & 96.88 \\
\hline Hole 7, Area-2 & 100 & 94.55 \\
\hline *Hole 8, Area-2 & 92.5 & 100 \\
\hline Average, Area-2 & $\mathbf{9 3 . 3 3}$ & $\mathbf{9 7 . 1 4}$ \\
\hline
\end{tabular}




\section{Analysis on Field Testing Data}

\begin{tabular}{|c|c|c|}
\hline Hole 14, Area-3 & N/A & 84.19 \\
\hline *Hole 15, Area-3 & N/A & 87.69 \\
\hline Hole 16, Area-3 & N/A & 93.43 \\
\hline Average, Area-3 & N/A & $\mathbf{8 8 . 4 4}$ \\
\hline Hole 9, Area-4 & 100 & 82.8 \\
\hline *Hole 10, Area-4 & 100 & 86.16 \\
\hline *Hole 16, Area-4 & 100 & 80.54 \\
\hline Average, Area -4 & $\mathbf{1 0 0}$ & $\mathbf{8 3 . 1 7}$ \\
\hline
\end{tabular}

Legend: * Holes used for building group data or training data

The hit rates of the classified and predicted rock types, which were picked up from Table IV-1, were presented in Tables 32 and 33. Table 32 are the classification/prediction results from using the group data obtained from Area-4 and Table 33 are the classification/prediction results from using the group data obtained from Area-1, 2, 3 and 4. Table 32 show that holes 1, 2, and 5 drilled in Area-1, the hit rates of the predicted gray shale and gray sandstone range from 100 to $100 \%$ and from 9.32 to $75.4 \%$, respectively. The average hit rates for the two rock types are about $100 \%$ and $41.16 \%$ respectively. For holes 6, 7 and 8 drilled in Area-2, the hit rates of the predicted gray shale and gray sandstone range from 100 to $100 \%$ and from 0 to $84.16 \%$ respectively. For holes 14, 15, and 16 drilled in Area-3, all the data were predicted into gray shale instead of gray sandstone. For holes 9, 10 and 16, the hit rates of the classified gray shale and gray sandstone range from 97.93 to $100 \%$ and 81.21 to $91.19 \%$, respectively.

As a comparison, building group data for each rock type by selecting Hole 5 from Area-1, Hole 8 from Area-2, Hole 15 from Area-3, and Holes 10 and 16 from Area-4 instead of selecting holes solely from Area-4 can improve the prediction/classification results significantly. Table 33 shows that, after using this group data to predict rock type, the hit rates obtained from Holes 1, 2 and 5 in Area-1, Hole 6 in Area-2, and Holes 14, 15, and 16 in Area-4 were greatly increased, from less than $50 \%$ to more than $78 \%$.

It can be concluded from the analysis on the data obtained from Mine A and Mine B that, under an ideal geological condition, the rock properties do not change much like the test site at Mine A, discriminant analysis method could work well in classifying and 


\section{Analysis on Field Testing Data}

predicting rock types. However, if rock properties vary significantly while drilling in different entry locations, the pre-built group data for a certain rock type is not able to satisfy the prediction (Area-1, 2, 3 and 4, Mine B). It should be updated by adding new drilling data sets obtained from the new locations (Tables 32 and 33). In other words, if the group data can not get updated in time while the geological conditions vary significantly, the result from the discriminant analysis will yield enormous and unacceptable errors. Another shortcoming is that this method needs core log in order to know the rock types and assign them to different rock layers. Furthermore, if there were no core log or scope data, the rock types over that roof will not be available, thus the rock type can not be assigned to the data points. Consequently, the requirement of using supervised discriminant analysis, which include the core log or scope data for building the group data for each rock type and update of the group data, limit the applicability of this technology in the field. 


\section{Summary and Conclusions}

Detecting the ever-changing geological properties of the entry roof is always a major objective for geologists and engineers responsible for roof bolting design. In this dissertation, a new derived drilling parameter, drilling hardness, was developed based on the drilling mechanical model (DMM) by considering energy lost and bit geometry. The following lists the summaries and conclusions in accordance with the laboratory tests, field tests and theoretical research results:

(1) The drilling parameters, such as thrust, torque and penetration rate, are affected by rock properties, the interaction between the drill bit and rock, and the hydraulic system. Among the three major factors, the factor that can be mitigated is the energy lost in the interaction between the drill bit and the rock. The tests conducted in the laboratory show that during the drilling process the total energy lost in both rotational and drilling directions is around 20 to $30 \%$ of the total mechanical energy done by the thrust and torque. In the rotational direction, 2 to $8 \%$ is lost in the torsion of the drill rod, and 0 to $0.8 \%$ is lost in the kinetic energy. In the drilling direction, about $1 \%$ is lost in kinetic energy, 2 to $8 \%$ is lost in potential energy, and 10 to $20 \%$ is lost in overcoming the friction between the drill bit and the drilled rock. Those lost energies negate the use of any original drilling parameters (thrust, torque, rotation rate, and penetration rate) for prediction.

(2) Data filtering and smoothing are indispensable for data processing in this research The applied data filtering and smoothing technologies, to a certain degree, eliminate some noise from the monitored drilling parameters.

(3) The friction between the drill bit and rock exists and varies with different rock types. Based on the energy equilibrium in rotational direction, $\mathfrak{t}$ can be reliably determined without considering the contact area between the drill bit and rock in rotational direction. 


\section{Summary and Conclusion}

(4) The laboratory test confirms Evans and Pomeroy's laboratory test result, the frictional coefficient between the drill bit and rock does change as the applied normal load varies. In addition, it is found from the laboratory test that the frictional coefficient keeps a good linear relationship with the applied torque.

(5) The drilling mechanical model (DMM), used to derive the new drilling parameter, drilling hardness, was founded. By applying this drilling mechanical model, the drilling hardness was derived based on the principle of energy equilibrium. This new drilling parameter reflects the resistance to indentation to the drill bit under an applied stress. Drilling hardness also shows the consistency of the resistance from rock within one rock layer and the difference of the resistance from different rock layers. The corresponding slope indicates the variance degree of the drilling hardness.

(6) The results obtained from the laboratory test show that drilling hardness can map the variable resistance to indentation to the drill bit while drilling in different rock layers. This feature of the drilling hardness makes it possible to detect the locations of interfaces between rock layers and discontinuities within one rock layer and to map rock properties using the monitored drilling parameters.

(7) The criteria used to determine the locations of interfaces and discontinuities were founded by applying statistics technology. If the peak value of the slope at location y is larger than or equal to the outer fence, y will be identified as the location of an interface. If the value of the negative slope at location y is within the outer and inner fences, $y$ will be identified as the location of a discontinuity, regardless it is a fracture or a crack. If there is more than one interface detected within 4 inches, the interface around that area can be determined by averaging the locations of the interfaces. In addition, it is necessary to ignore the interfaces derived from the first 5 inches (from the roof line) and the last 2 inches.

(8) By applying the DMM to obtain the corresponding drilling hardness and slope for the holes drilled in the field, it is also found that the pre-setting of thrust cap, penetration rate, rotation rate affect the result of drilling hardness. The analysis results from the laboratory tests show that pre-setting higher thrust cap, and allowing either penetration rate or rotation rate free (not fixed simultaneously) can 


\section{Summary and Conclusion}

yield much better results in roof geological mapping - the slope of the drilling hardness could more easily reflect the locations of the interfaces and discontinuities.

(9) The results from the field test conducted at Mine A shows that it is reliable and accurate in determining the locations of interfaces between rock layers with average error 1.37 inches by using the drilling hardness and slope. However, the accuracy in determining the locations of discontinuities within one rock layer is not conclusive, because it is hard to compare the derived and observed and core logged locations of discontinuities. This is due to the fact that some of the results from the scope and core log still show some differences. Nevertheless, the error for the discontinuities shown in both the observed from the scope and the derived from the drilling hardness is very small, and the average error is 1.32 inches.

(10) The results from the field test conducted at Mine B also show that the drilling hardness and slope are still reliable and accurate in determining the locations of interfaces between rock layers with average error 1.22 inches in the four testing areas. The analysis results from this mine also show that the derived location of the interface from the drilling hardness in different areas basically reflects the variation in roof geology from Area- $4 \rightarrow$ Area- $1 \rightarrow$ Area-2 $\rightarrow$ Area-3 (Figure 80).

(11) Compared with core $\operatorname{logs}$ in the four areas at Mine B, only $25.86 \%$ of discontinuities are identified and derived from the drilling hardness with the maximum, minimum and average errors $2.98,0.04$, and 1.481 inches respectively. These discontinuities shown in the core log but not in the derived data can be considered as the ones that are not weak enough in strength to be detected using the slope of the drilling hardness.

(12) By applying the technology of supervised discriminant analysis, the algorithms of rock type classification/prediction were developed by using thrust, penetration rate, torque, rotation rate and drilling hardness.

(13) The rock type classification/prediction results obtained from the tests carried out at Mine A show that for the holes used for testing the prediction result, the hit rates of the predicted gray shale and gray sandstone range from 83.33 to $97.24 \%$ and from 78.8 to $95.87 \%$ respectively. The average hit rates for the two rock types 


\section{Summary and Conclusion}

are $91.23 \%$ and $87.51 \%$ respectively. For these holes used for testing the classification result, the hit rates of the classified gray shale and gray sandstone range from 89.71 to $100 \%$ and from 91.6 to98.37\% respectively. The average hit rates for the two rock types are about $96.25 \%$ and $95.25 \%$ respectively.

(14) The field tests, particularly the test conducted at Mine B, show that the requisites, which include the core log or scope data for building the group data for each rock type and update of the group data if roof geology changes significantly, limit the applicability of this technology and make it not feasible in applying this discriminant analysis in the field. 


\section{$\underline{\text { References }}$}

\section{References}

1. Maurer, W.C., Novel Drilling Techniques, Pergamon Presss Ltd., Britain, 1968, pp.114.

2. Teale, R., "The Concept of Specific Energy in Rock Drilling," Int. J. Rock Mech. Mining Sci. Vol. 2, Pergamon Press 1965, pp.57-73.

3. Takach, T., Morris, S., and Miller, G., "Control System for Roof Drilling", Parvus Corporartion, Salt Lake City, Utah, U.S. Bureau of Mines, Spokane Research Center, Spokane, WA, pp. 1-10.

4. Signer, S. P., King, R. L., Evaluation of Coal Mine Roof Support Using Artificial Intelligence, Spokane Research Center, U. S. Bureau of Mines, Spokane, Washingwon, chapter 86.

5. King, R. L., Hicks, M. A., Signer, S. P., "Using Unsupervised Learning for Feature Detection in a Coal Mine Roof," Engng Applic. Artif. Intell. Vol.No. 6, 1993, pp. $565-573$.

6. Utt, W. K., Neural Network Technology for Strata Strength Characterization, Spokane Research Laboratory, NIOSH, Spokane, WA, Presented at the International Joint Conference on Neural Networks in Washington, DC, July 1016, 1999.

7. Thomas, B. and Wilson, G., "Control Technology for Roof Drill Operator," $18^{\text {th }}$ conference on Ground Control in Mining, Aug. 3-5, 1999, pp.216-221.

8. Simonoff, J. S., Smoothing Methods in Statistics, Springer-Verlag New York, Inc., 1996.

9. Hart. J.D., Nonparametric Smoothing and Lack-of-Fit Tests, Springer-Verlag New York, Inc., 1997.

10. Finfinger, G., Peng, S. S., Gu, Q., Wilson, G. and Thomas, B., “An Approach to Identify Geological Properties from Roof Bolter Drillin Parameters," $19^{\text {th }}$ Conference on Ground Control in Mining, Aug., 2000, pp. 1-11. 


\section{$\underline{\text { References }}$}

11. Reddish, D. J., and Yasar, E., "A New Portable Rock Strength Index Test Based on Specific Energy of Drilling," Int. J. Rock Mech. Min. Sci. \& Geomech. Abstr. Vol. 33. No. 5, pp. 543-548, 1996.

12. Jain, A. K., and Singh, D. P., Specific Energy as a Criterion for Drillability of Rocks - A Laboratory Study, Geotechnical Instrumentation and Monitoring in open Pit and Underground Mining, Szwedzicki (ed.), 1993, Balkema, Rotterdam, ISBN 905410321 3, pp. 253-263.

13. Stefanko, R., Coal Mining Technology Theory and Practice, Published by Society of Mining Engineers of the American Institute of Mining, Metallurgical, and Petroleum Engineers, Inc., New York, 1983.

14. Itakura, K., Sato, K., Deguchi, G., Icihara, Y. and Matsumoto, H., Estimation of Roof Structure by Mechanical Data Logging System for Roofbolter, Muroran Institute of Technology, Japan Coal Energy Center (JCOAL), Kushiro Colliery, Taiheiyo Coal Mining Co. Ltd., Coal \& Safety No. 13, Sept., 1998, pp. 42-56.

15. Itakura, K., Sato, K., Deguchi, G., Icihara, Y. Matsumoto, H. and Eguchi, H., Development of Roof Logging System by Rock Bolt Drilling, Muroran Institute of Technology, The Coal Mining Research Center, Japan, Kushiro Colliery, Taieiyo Coal Mining co., Ltd., trans. Instn Min. Metall, Vol. 106, Sect. A, 1997, pp.118.123.

16. Itakura, K., Sato, K., Deguchi, G., Icihara, Y. Matsumoto, H., "Visualization of Geostructure by Mechanical Data Logging of Rockbolt Drilling and Its Accuracy," $20^{\text {th }}$ International Conference on Ground Control in Mining, Aug. 2001, pp. 184-190.

17. Peng, S.S., Luo, Y., and Finfinger, G., Evaluation of Roof Bolting Requirements Based on In-mine Roof Bolter Drilling Parameters, IOF Bolter proposal, Dept. of Mining Engineering, West Virginia University, 1999.

18. Fausett. L., Fundamentals of Neural Networks, Florida Institute of Technology, Prentice Hall. Englewood Cliffs. NJ 07632, 1993.

19. Pessier, R. C. and Fear, M. J., "Quantifying Common Drilling Problems with Mechanical Specific Energy and a Bit-Specific Coefficient of Sliding Friction,” 


\section{$\underline{\text { References }}$}

$67^{\text {th }}$ Annual Technical Conference and Exhibition of the Society of Petroleum Engineers, Oct. 4-7, 1992, pp. 373-387.

20. Gu, Q., Rock Type Classification Using Discriminant Analysis, Group Meeting Presentation, Dept. of Mining Engineering, West Virginia University, April, 2001.

21. Gu, Q.., Determination of Rock Properties, Presentation of Seminar, Dept. of Mining Engineering, West Virginia University, Oct. 31, 2001.

22. Huberty, C.J, Applied Discriminant Analysis, A Wiley-Interscience Publication, John Wiley \& Sons, Inc., 1994, pp. 39-276.

23. Drilling in Feedback/Auto, Confidential Document, J.H.Fletcher \& Co., 2001.

24. Vanguard Software Corporation, Data Smoothing, Retrieved 2000, from http://www.vanguardsw.com/dphelp/dph00161.htm.

25. Sampling Techniques, Basic Concepts of Sampling, Retrieved 1996, from http://cne.gmu.edu/modules/dau/stat/data/sample_bdy.html.

26. Exponential Smoothing, Engineering Statistics Handbook, from http://www.itl.nist.gov/div898/handbook/pmc/section4.

27. Sampling Methods, StatPac Inc, Retrieved 2000, from http://www.statpac.com/surveys/sampling.htm.

28. Gu, Q., Geological Mapping of Entry Roof in Mines, Proposal defense, Dept. of Mining Engineering, West Virginia University, Feb. 13, 2002.

29. Harner, E. J., Handout of Course Stat341, Dept., of Statistics, West Virginia University, 2001.

30. Evans, I., and Pomeroy, C.D., The Strength, Fracture and Workability of Coal, Pergamon Press Ltd., 1966, pp.170-182.

31. Bowden, F. P. and Tabor, D., The Friction and Lubrication of Solids, Oxford University Press, amen House, London E. C. 4, 1964, pp. 99-100.

32. Han, J., Identify Strata Geological Features Using Color Borehole Camera Scope System, Presentation of Seminar, Dept. of Mining Engineering, West Virginina University, Feb. 28, 2003. 


\section{Appendix I}

\section{Calculation Procedure for Determining Interfaces and Discontinuities}

In this section, the detailed calculation procedure of the drilling hardness and its slope is presented. After converting the measured machine data into the raw data, it is ready to do the following calculations. The raw data consists of five drilling parameters, thrust $(\mathrm{F})$, penetration rate $(\mathrm{u})$, torque (T), RPM, and the bit position (d). Figure I- 1 shows the flow chart of this calculation procedure.

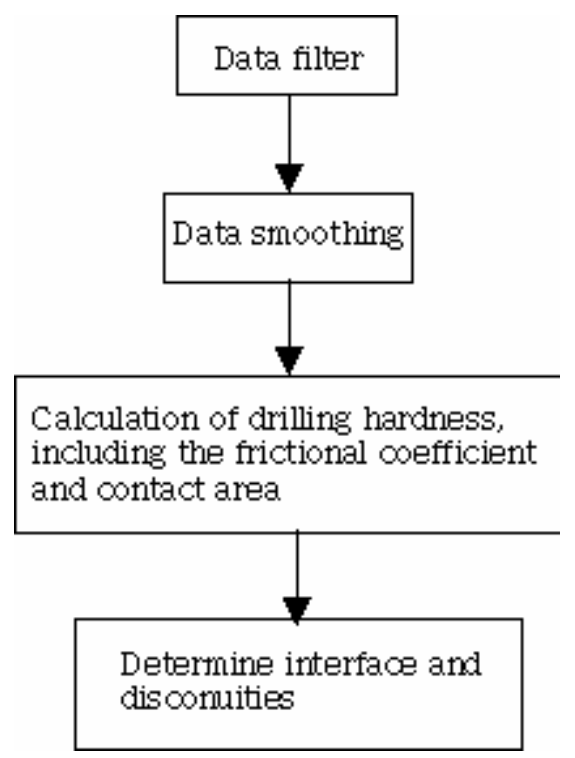

Figure I-1 Flow chart of the calculation procedure

(1) Data filtering

If the number of monitored data sets that share the same position is greater than two, the first two monitored data sets will be averaged, and the remaining data sets sharing the same position will be filtered out. This data filtering technique is only applied to the raw data converted from the machine data. This filtered data are called FD. 


\section{Appendix I}

(2) Data smoothing and sampling

In this session, the Nadaraya-Watson with Gaussion kernel method is employed

$$
Y_{\text {smooth }}(j)=\frac{\sum_{i=1}^{n} y_{\text {ori }}(i) \cdot K((D(j)-D(i)) / h)}{\sum_{i=1}^{n} K\left(\frac{D(j)-D(i)}{h}\right)}
$$

where $y_{\text {ori }}$ - the filtered data (FD)

$\mathrm{Y}_{\text {smooth }}$ - the smoothed data

$\mathrm{K}$ - a function called the kernel. A popular choice of kernel is the Gaussian kernel,

$$
K(x)=\frac{1}{\sqrt{2 \pi}} \exp \left(-\frac{x^{2}}{2}\right)
$$

$\mathrm{h}-$ the bandwidth, $h=1.059 \cdot \sigma \cdot n^{-\frac{1}{5}}$

$\sigma$ - the standard deviation of drilling distance for a specific drilled hole

$\mathrm{n}$ - the number of data sets obtained in one drilled hole

$\mathrm{N}$ - the number of data sets that are around the data set $\mathrm{j}$

$\mathrm{j}$ - refers to the smoothed data set

$\mathrm{i}$ - refers to the original data set

$\mathrm{D}(\mathrm{j})$ - bit position at point $\mathrm{j}$.

After data smoothing, the smoothed data (SD) are obtained.

(3) Data sampling (optional)

In this session, the systematic sampling is employed. The data sets of $\mathbf{S D}$ can be sampled periodically, using either the odd sets or the even sets. After this step, the smoothed and sampled data (SSD) are obtained. 


\section{Appendix I}

(4) Determination of the frictional coefficient

The frictional coefficient $f_{i}$ at point $i$ is calculated by

$$
f_{i}=\frac{\tau_{i} \cdot \theta_{i}-\frac{1}{2} I \cdot \omega_{i}{ }^{2}+\frac{1}{2} I \cdot \omega_{i-1}^{2}+\frac{\tau_{i}^{2} \cdot L}{G \cdot I_{p}}}{F_{i} \cdot R \cdot \theta_{i}}
$$

where $f_{i}-$ the frictional coefficient at point $i$

$\theta_{\mathrm{i}}-$ rotated angle from point $\mathrm{i}-1$ to $\mathrm{i}, \theta=\omega_{i-1} \cdot t+\frac{1}{2} a_{i} \cdot t^{2}$

$\alpha_{\mathrm{i}}-$ angular acceleration at point $\mathrm{i}, \mathrm{rad} / \mathrm{s}^{2}, \alpha_{i}=\frac{2 \pi}{60 \cdot t} \cdot\left(R P M_{i}-R P M_{i-1}\right)$

$\omega_{\mathrm{i}}-$ angular velocity at point $\mathrm{i}, \mathrm{rad} / \mathrm{s}, \omega_{i}=\frac{R P M_{i}}{60} \cdot 2 \pi$

$\tau_{\mathrm{i}}$ - measured torque at point $\mathrm{i}$

$\mathrm{L}$ - length of the drill rod, 62 inch

$\mathrm{G}-$ shear modulus of the rod, $11.5 \times 10^{6}, \mathrm{lb} / \mathrm{in}^{2}$

I - the mass moment of inertia of drill rod along the rotation axis

$$
I=0.083 \cdot m\left(r_{1}^{2}+r_{2}^{2}\right), \mathrm{lb}-\mathrm{in}^{2}
$$

$\mathrm{m}-$ mass of the rod

Ip - the moment of inertia of drill rod and bit along the rotation axis

$$
I_{p}=1.9246\left(r_{2}^{4}-r_{1}^{4}\right)
$$

$r_{1}, r_{2}-$ the inner and outer radius of the drill rod, $r_{1}=0.271$ inch, $r_{2}=0.396$ inch

$\mathrm{R}$ - the outer radius of the drill bit, 0.6875 inch (1 3/8 bit)

(5) Determination of the contact area between the drill bit and rock 


\section{Appendix I}

The varied contact area with the changing of penetration per revolution between the drill bit and the rock is estimated by the following expressions.

For the $13 / 8$ drill bit

$$
A_{i}=\left\{\begin{array}{l}
1.1518 \cdot p_{-} r_{i}, \text { if } p_{-} r_{i} \leq 0.125^{\prime \prime} \\
1.1518 \cdot p_{-} r_{i}+18.04762 \cdot\left(p_{-} r_{i}-0.125\right)^{2}, \text { if } 0.28125^{\prime \prime} \geq p_{-} r_{i} \geq 0.125^{\prime \prime}
\end{array}\right.
$$

where, $\mathrm{p} \__{\mathrm{i}}-$ bite depth per revolution, in/rev.

For the $11 / 32$ drill bit

$$
A_{i}=\left\{\begin{array}{l}
1.0607 \cdot p_{-} r_{i}, \text { if } p_{-} r_{i} \leq 0.125^{\prime \prime} \\
0.13258+1.32583 \cdot\left(p_{-} r_{i}-0.125\right), \text { if } 0.28125^{\prime \prime} \geq p_{-} r_{i} \geq 0.125^{\prime \prime}
\end{array}\right.
$$

where, $p_{-} r_{i}-$ bite depth per revolution, in/rev.

(6) Determination of drilling hardness

The drilling hardness is calculated by the following equation

$$
S_{i}=\frac{F_{i} \cdot d_{i}\left(1-c \cdot f_{i}\right)-0.5 \cdot m \cdot\left(\left(p r_{i}\right)^{2}-\left(p r_{i-1}\right)^{2}\right)-m g \cdot d_{i}}{d_{i} \cdot A_{i}}
$$

where $S_{i}-$ calculated drilling hardness (the new drilling parameter) at position $i, p s i$

$\mathrm{F}_{\mathrm{i}}$ - measured thrust at point $\mathrm{i}, \mathrm{lbs}$

$\mathrm{m}$ - mass of the drill bit, rod, mast and roof header

$\mathrm{pr}_{\mathrm{i}}$ - measured penetration rate at position $\mathrm{i}, \mathrm{in} / \mathrm{sec}$

$\mathrm{d}_{\mathrm{i}}$ - distance drilled from point $\mathrm{i}-1$ to $\mathrm{i}$

$f_{i}$ - frictional coefficient between the front part of the drill bit and 


\section{Appendix I}

rock at position i. It's determined in step (4)

$\mathrm{A}_{\mathrm{i}}$ - contact area between the bit and the drilled rock, in ${ }^{2}$. It's determined in step

$F_{i} \cdot d_{i}$ - work done by thrust from point i -1 to i

$\frac{1}{2} m \cdot\left(\left(p r_{i}\right)^{2}-\left(p r_{i-1}\right)^{2}\right)-$ kinetic energy changed from point $\mathrm{i}-1$ to point $\mathrm{i}$

$c \cdot F_{i} \cdot f_{i} \cdot d_{i}$ - work used to overcome the frictional resistance in drilling direction $m g \cdot d_{i}$ - potential energy from point $\mathrm{i} 1$ to $\mathrm{i}$

$S_{i} \cdot A_{i} \cdot d_{i}$ - work used to break the cementing bond of the rock

(7) Determination of the slope of drilling hardness

By applying the data sets of the drilling hardness to the following equation, the corresponding slope is determined.

$$
\text { slope }(i)=\frac{n \cdot \sum_{j=1}^{n} x_{j} \cdot y_{j}-\left(\sum_{j=1}^{n} x_{j}\right)\left(\sum_{j=1}^{n} y_{j}\right)}{n \sum_{j=1}^{n} x_{j}{ }^{2}-\left(\sum_{j=1}^{n} x_{j}\right)^{2}}
$$

where slope $(\mathrm{i})$ - the slope of drilling hardness at point $\mathrm{i}$

$\mathrm{n}$ - the number of data points used to calculate the slope at point $\mathrm{i}$

$\mathrm{x}_{\mathrm{j}}-$ the drilling distance from hole mouth at point $\mathrm{ij}$ or $\mathrm{i}+\mathrm{j}$

$y_{j}-$ the value of drilling hardness at point $i-j$ or $i+j$

(8) Determination of outliers of the slope

Extreme outliers are the indicators of interfaces and suspect outliers are the indicators of fractures. 


\section{Appendix I}

- Arrange the data sets of slope in order of magnitude;

- Pick the lower quartile, $\mathrm{Q}_{1}$, which is the value of the slope in the position $0.25(\mathrm{i}+1)$. Then pick the upper quartile, $\mathrm{Q}_{3}$, which is the value of the slope in the position $0.75(i+1)$. When these positions are not integers, using the average values in the two adjacent positions;

- Calculate the inter-quartile range (IQR). IQR is the difference between the upper and lower quartiles, that is, $\mathrm{IQR}=\mathrm{Q}_{3}-\mathrm{Q}_{1}$.

- Calculate the inner and outer fences as follows:

Inner fences: $\mathrm{Q}_{1}-1.5 \times(\mathrm{IQR})$ and $\mathrm{Q}_{3}+1.5 \times(\mathrm{IQR})$

Outer fences: $\mathrm{Q}_{1}-3 \times(\mathrm{IQR})$ and $\mathrm{Q}_{3}+3 \times(\mathrm{IQR})$

- Determine the outliers.

Suspect outliers: $\mathrm{Q}_{3}+1.5 \times(\mathrm{IQR}) \leq$ slope $\leq \mathrm{Q}_{3}+3 \times(\mathrm{IQR})$ or

$$
\mathrm{Q}_{1}-1.5 \times(\mathrm{IQR}) \geq \text { slope } \geq \mathrm{Q}_{1}-3 \times(\mathrm{IQR})
$$

Extreme outliers: slope $\geq \mathrm{Q}_{3}+3 \times(\mathrm{IQR})$ or

$$
\text { slope } \leq \mathrm{Q}_{1}-3 \times(\mathrm{IQR})
$$

(9) Criteria of determining interface and discontinuity

The basic idea of using these outliers either above the outer fences or between the outer and inner fences is to determine the locations of interfaces between rock layers and discontinuities within one rock layer. In the following sections, the term discontinuity is used to specify the lack of continuity within one rock layer, such as fractures, cracks, etc. The criteria used in identifying an interface and a discontinuity are determined in the following three steps:

(4) Apply the basic criteria to the slope of drilling hardness for a drilled hole. The basic criteria are that if the value of the slope at location $y$ is larger than or equal to that of the outer fence, then y will be identified as the location of an interface. In addition, Figure 33 shows that there are at least one point near the interface whose value(s) of slope is (are) beyond the outer fence. Thus, the location of the peak value from these points whose values of slope are beyond the outer fence is determined as the location of the interface. If the peak value of the negative slope 
at location $\mathrm{y}$ is between the outer and inner fences, then y will be identified as the location of a discontinuity, regardless it is a fracture or a crack as observed from the scope or core log. The reason why only the negative slope is used in determining the location of discontinuities is that these discontinuities are weak in strength. When drilling through a discontinuity, usually the corresponding drilling hardness tends to decrease; thus the slope of the drilling hardness around that location is negative. The positive slope, at location y that is within the outer and inner fence, usually indicates that it is a harder sub-layer than its adjacent sublayers.

(5) If there is more than one interface detected within 4 inches (counting from the bottom to the top), the interface around that area can be determined by averaging the locations of the interfaces (Holes 2, 3, 4, 5, 6, 7, and 8 drilled on Block 6), but this averaged location can not compare with the next interface location.

(6) Ignore the interfaces and discontinuities derived from the first 5 inches (from the roof line) and the last 2 inches (hole end). The goal is to avoid the influence from the adjustment of the hydraulic system during startup and finish. 


\section{Appendix II}

\section{Analysis Results from Blocks 2, 3, and 4, Fractured Block, and Solid Block}

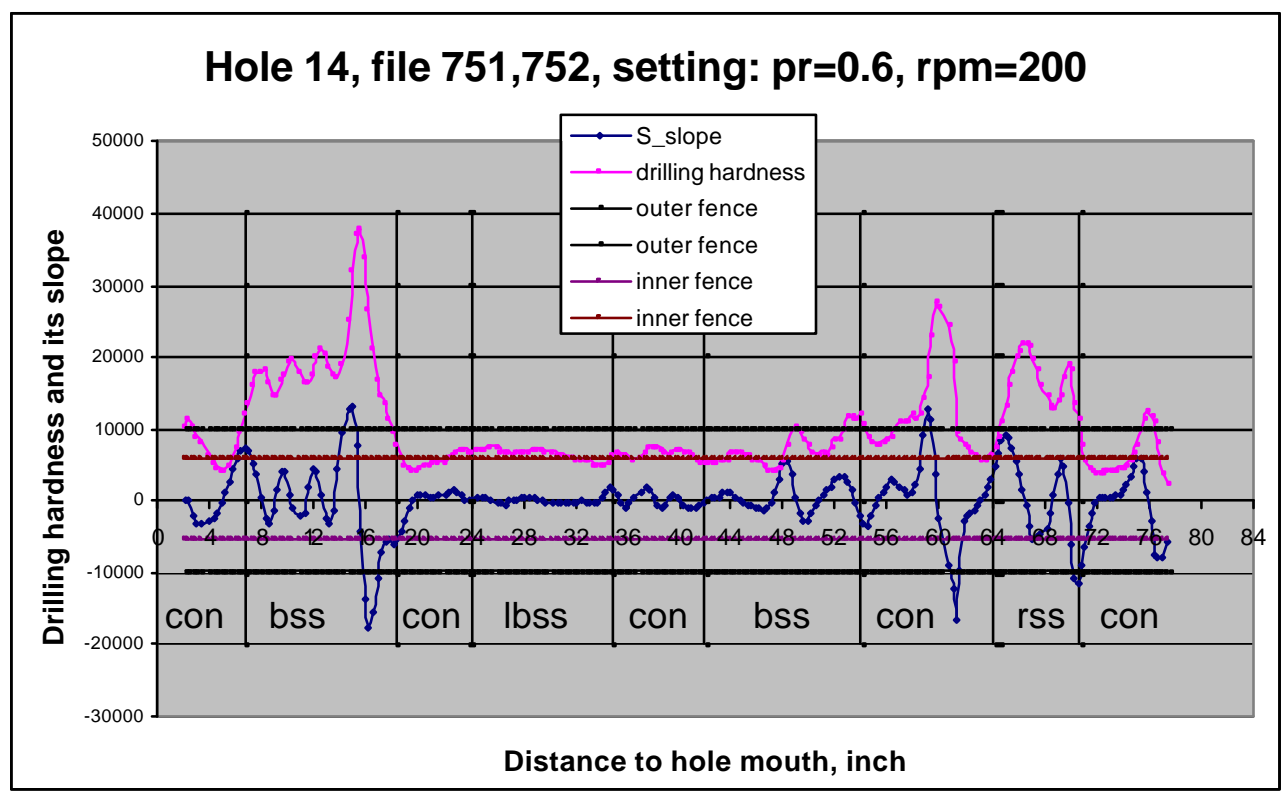

Figure II- 1 Drilling hardness and slope for Hole 14, Block 2

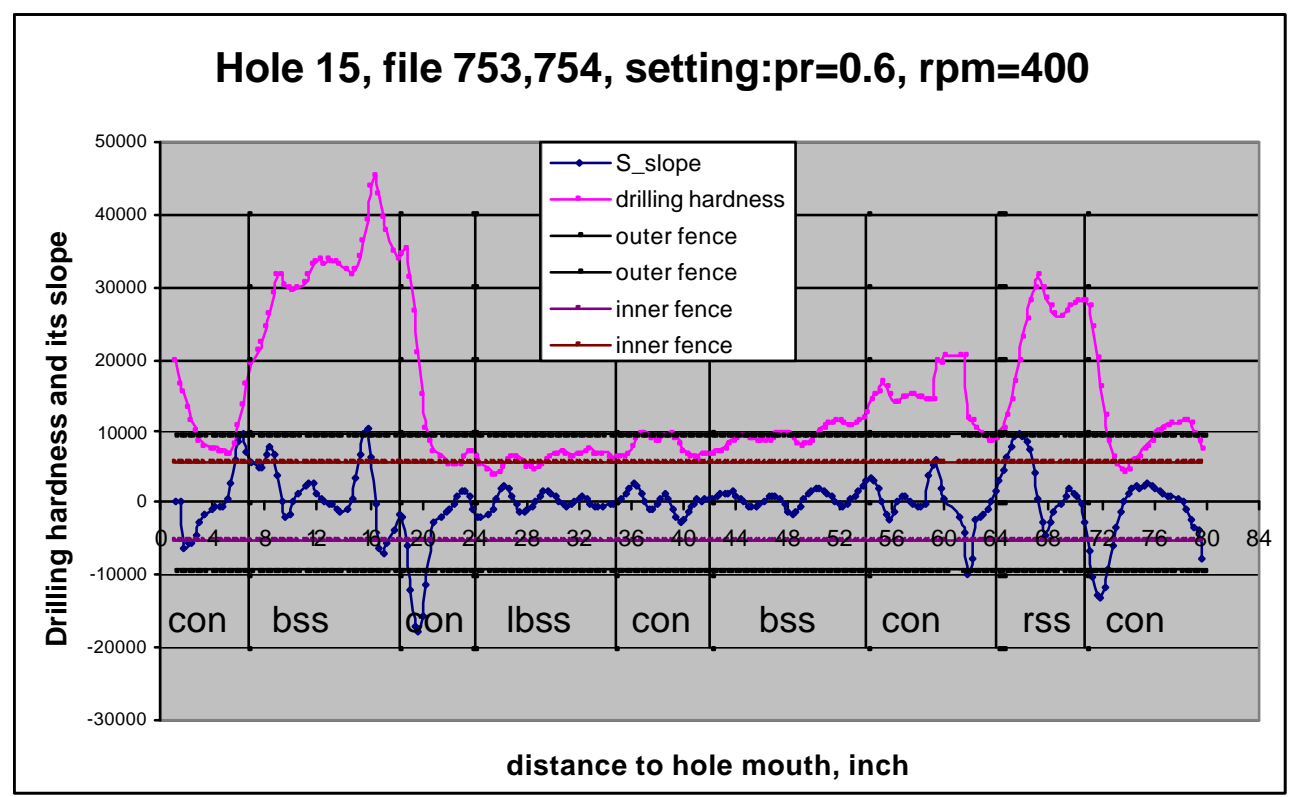

Figure II- 2 Drilling hardness and slope for Hole 15, Block 2 


\section{Appendix II}

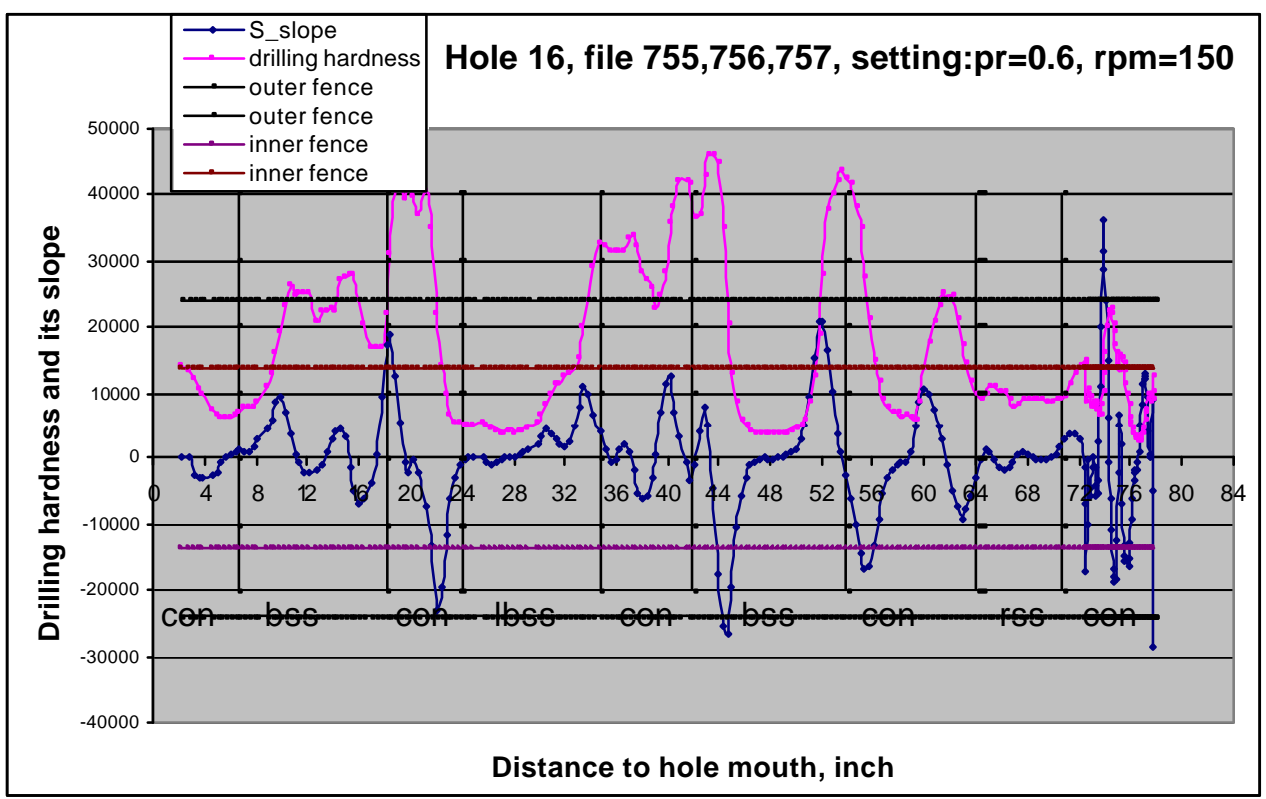

Figure II- 3 Drilling hardness and slope for Hole 16, Block 2

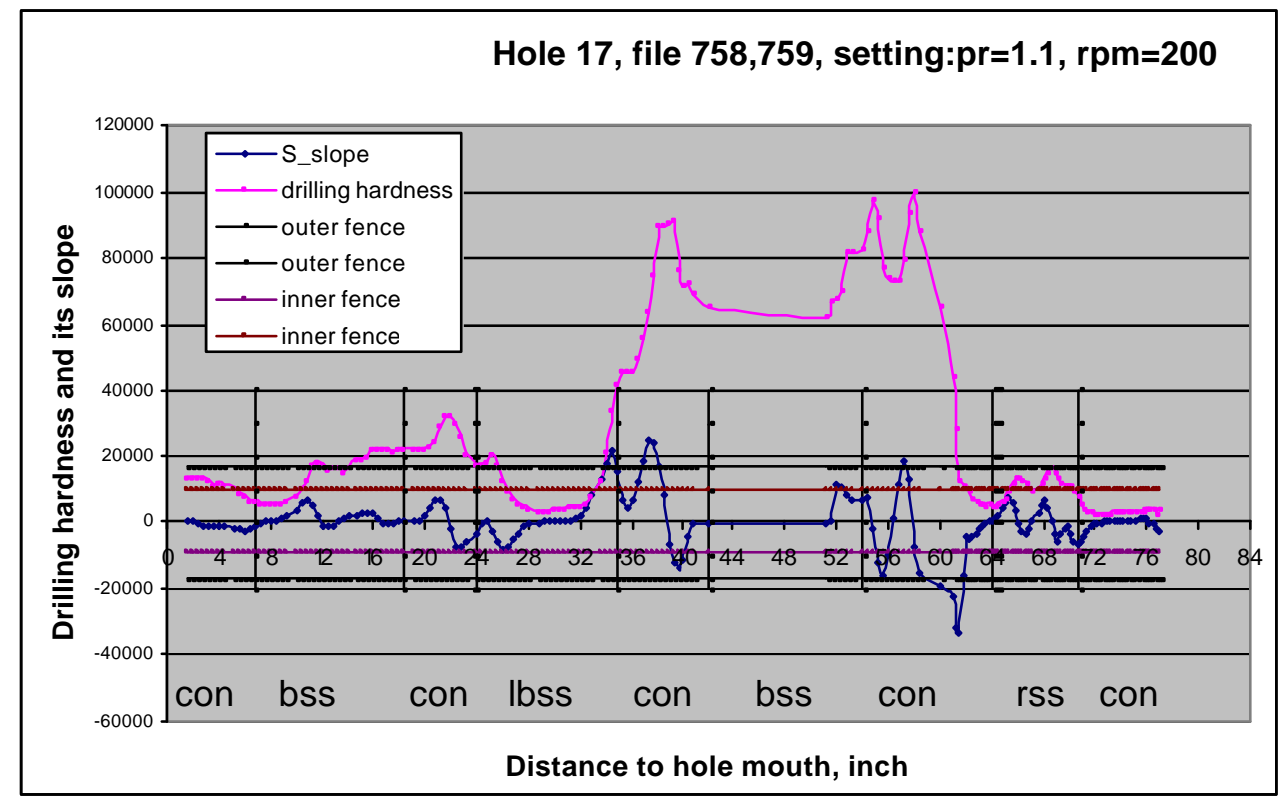

Figure II- 4 Drilling hardness and slope for Hole 17, Block 2 


\section{Appendix II}

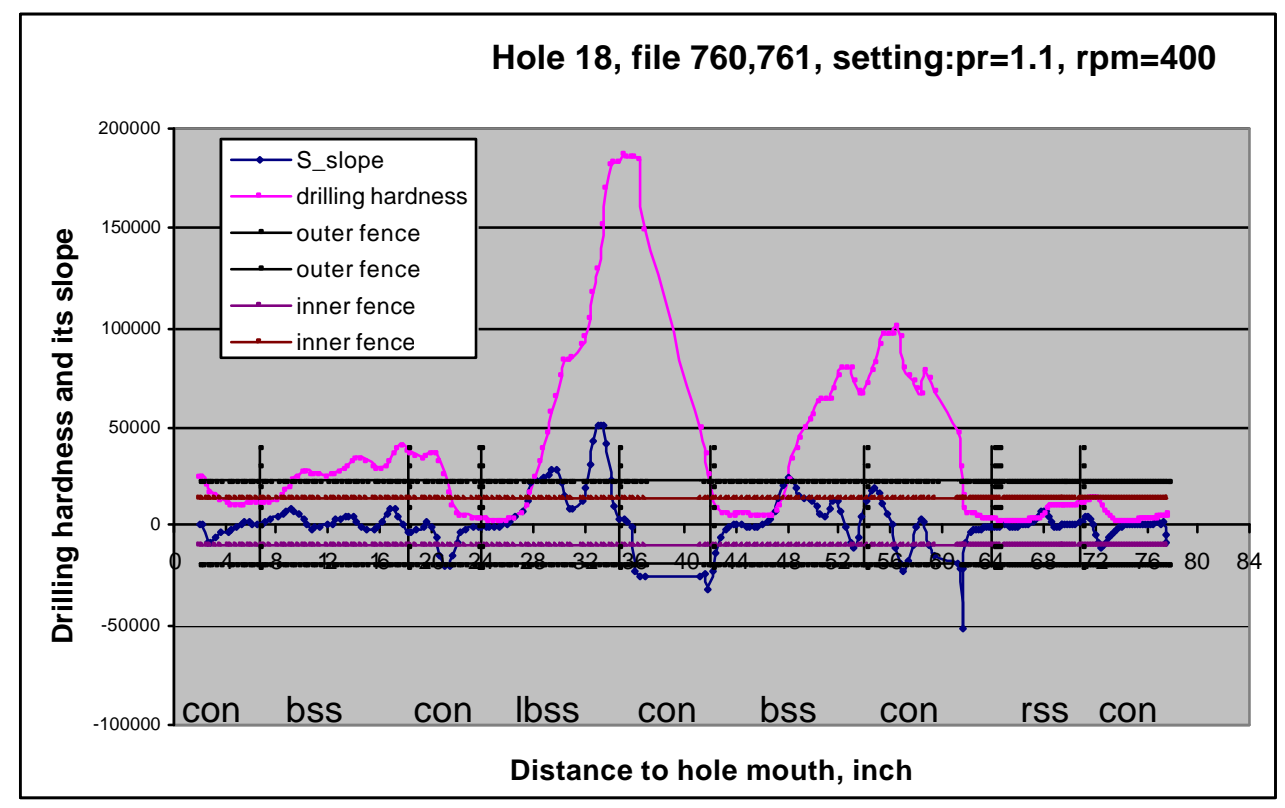

Figure II- 5 Drilling hardness and slope for Hole 18, Block 2

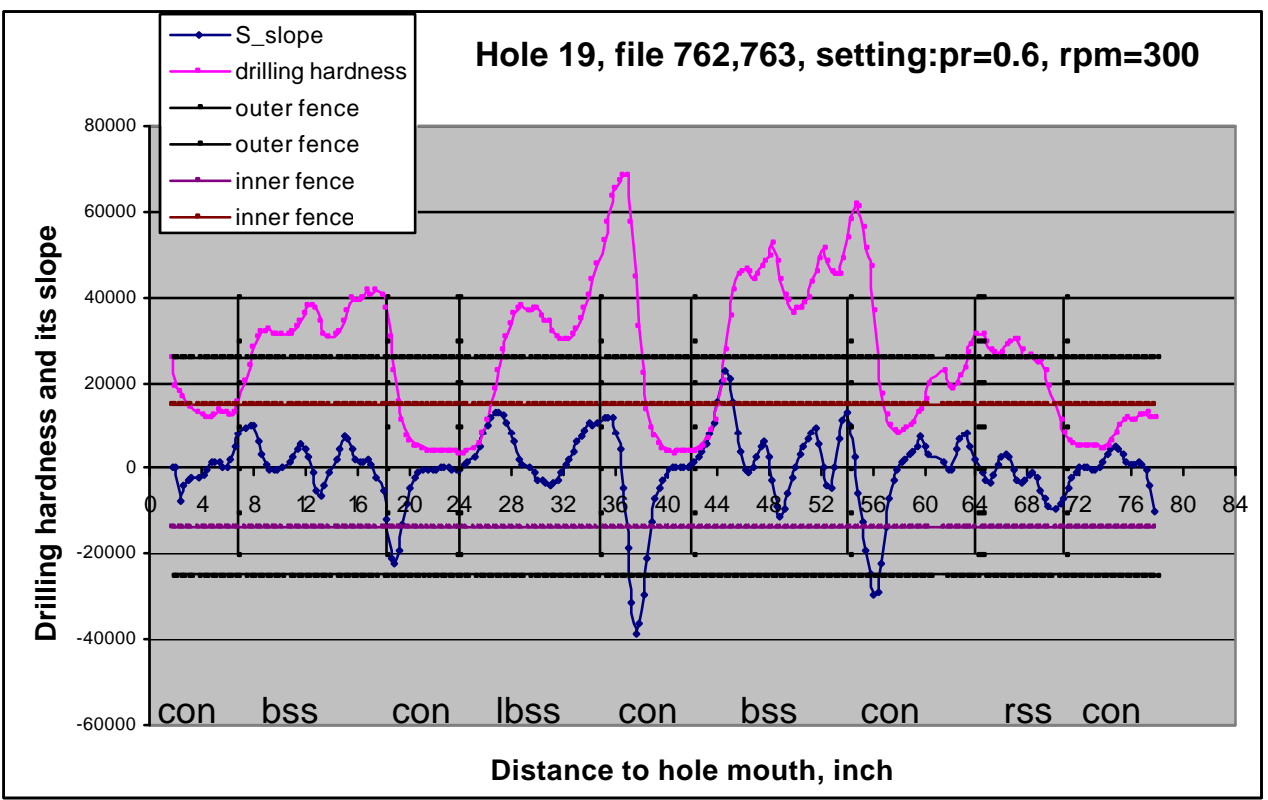

Figure II- 6 Drilling hardness and slope for Hole 19, Block 2 


\section{Appendix II}

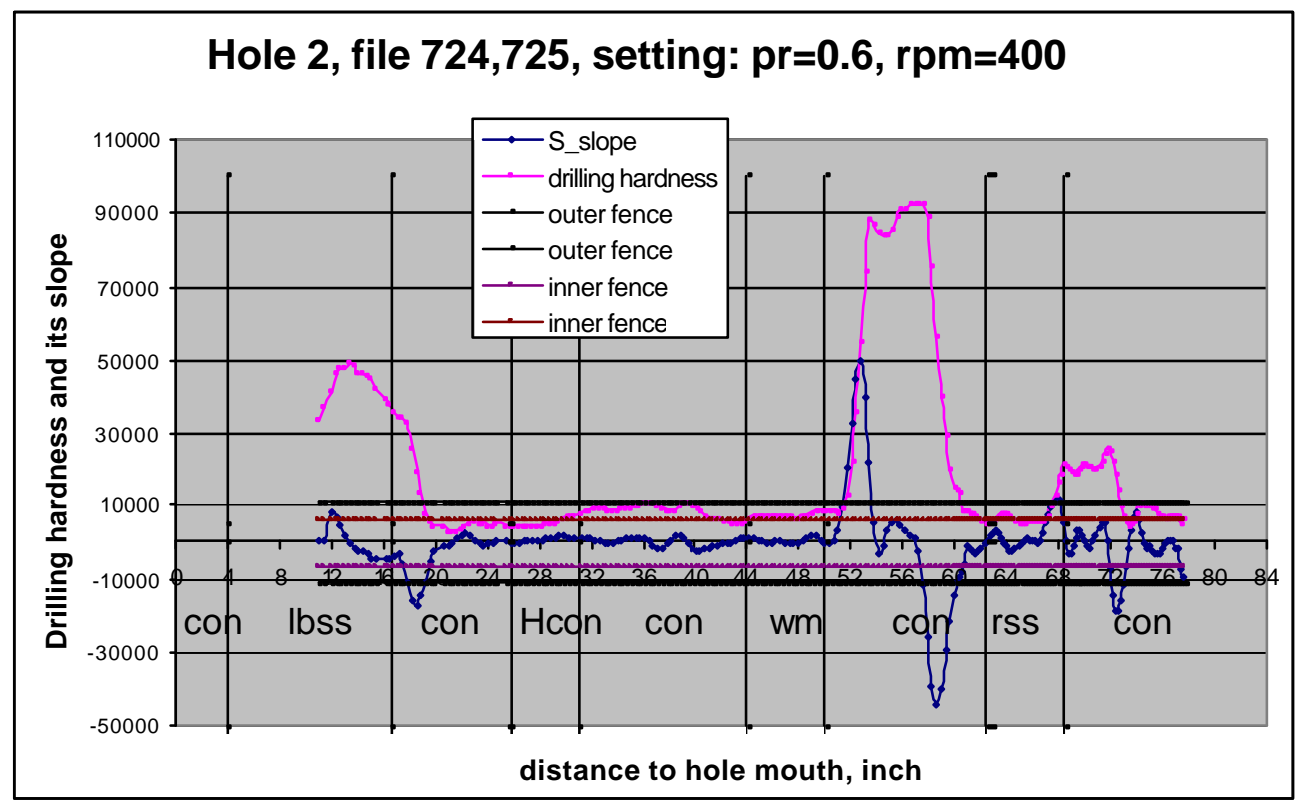

Figure II- 7 Drilling hardness and slope for Hole 2, Block 3

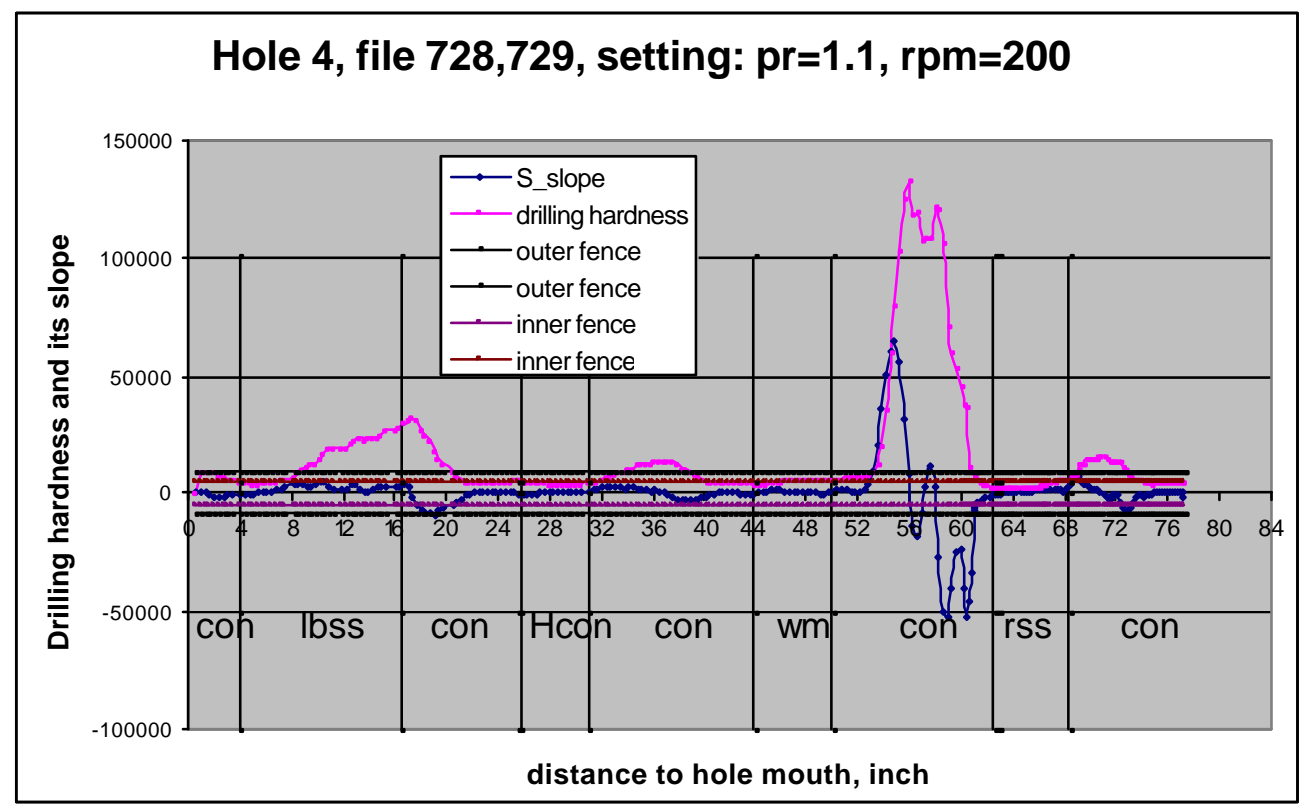

Figure II- 8 Drilling hardness and slope for Hole 4, Block 3 


\section{Appendix II}

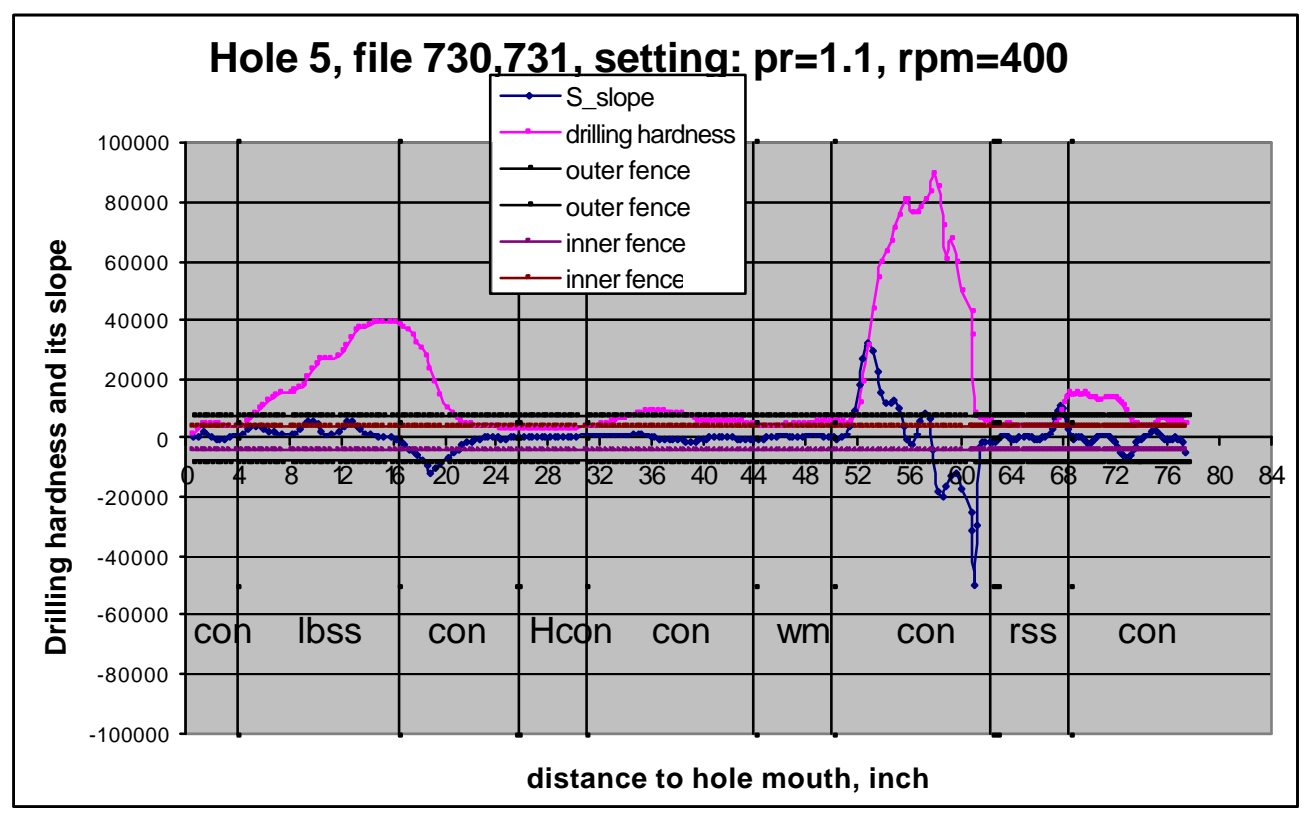

Figure II- 9 Drilling hardness and slope for Hole 5, Block 3

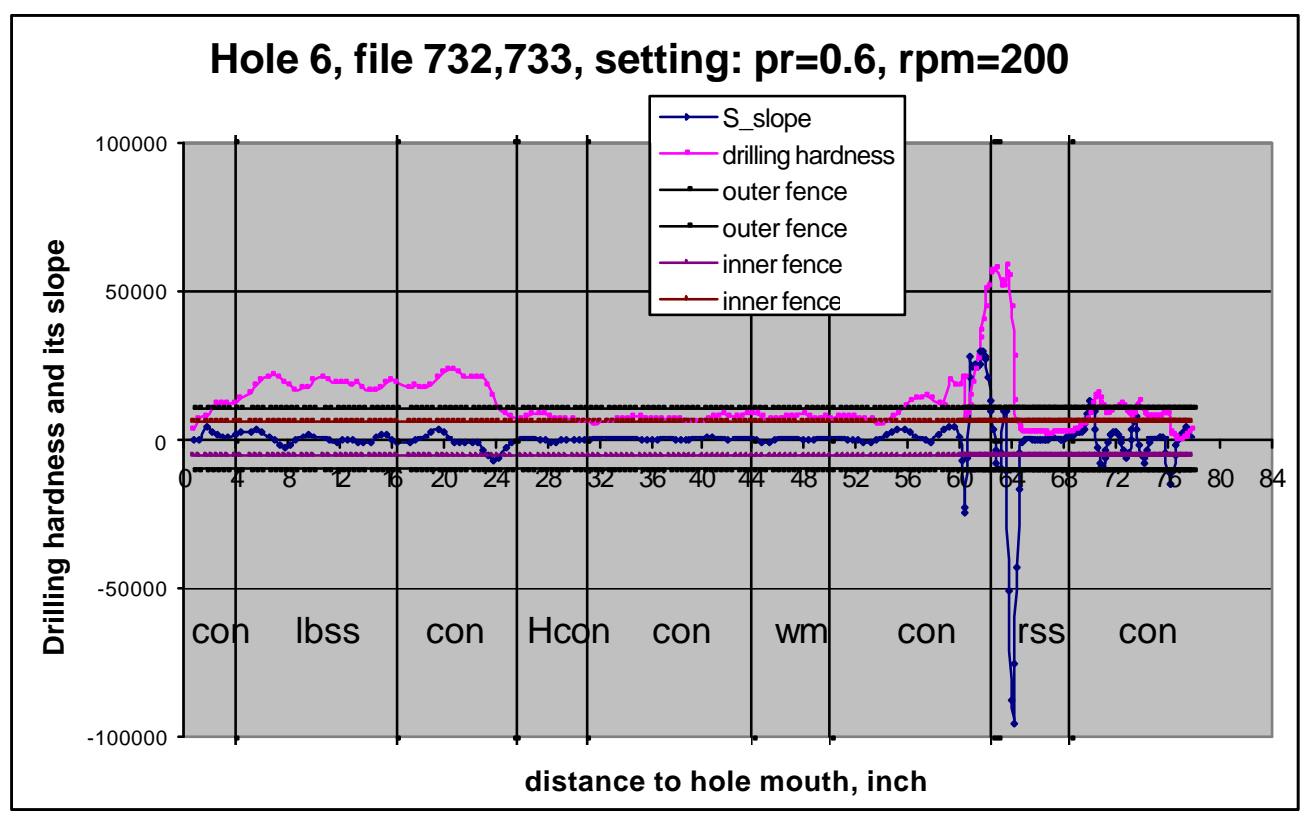

Figure II- 10 Drilling hardness and slope for Hole 6, Block 3 


\section{Appendix II}

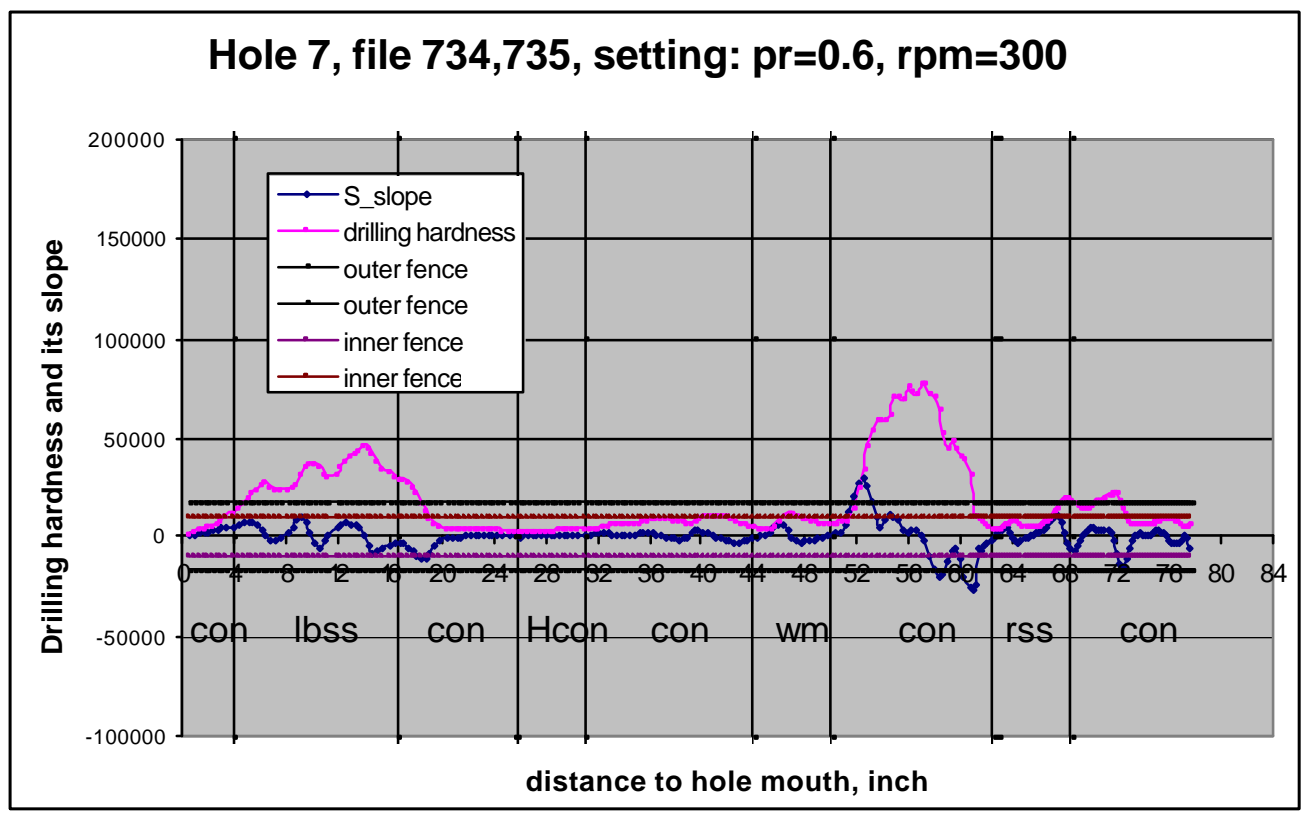

Figure II- 11 Drilling hardness and slope for Hole 7, Block 3

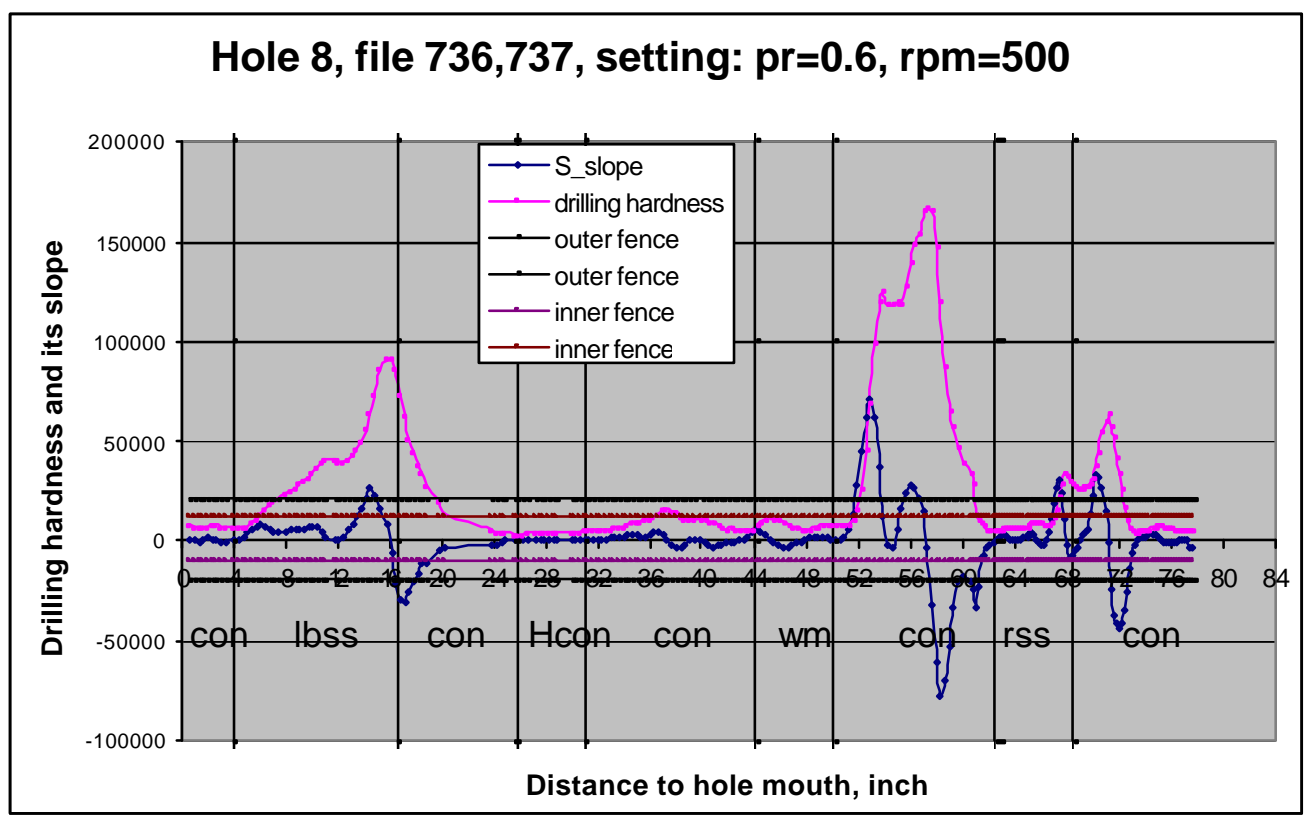

Figure II- 12 Drilling hardness and slope for Hole 8, Block 3 


\section{Appendix II}

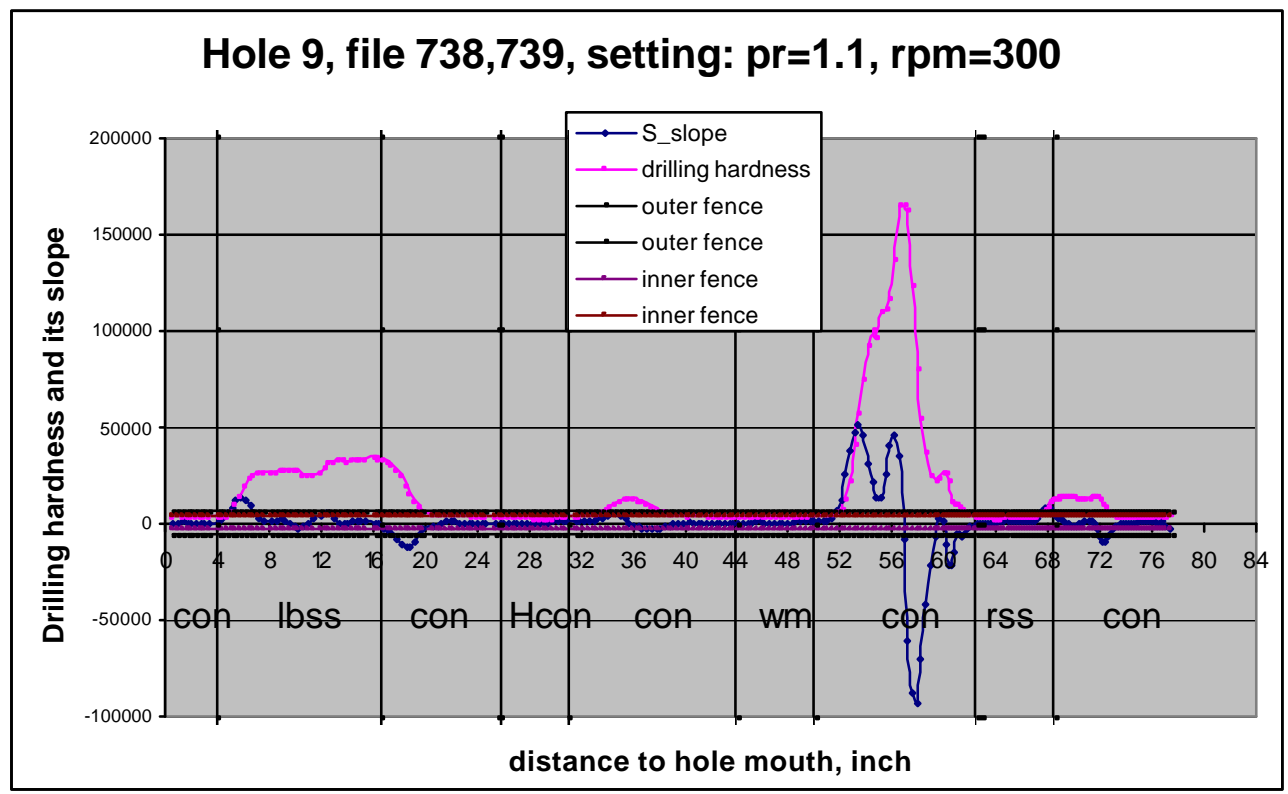

Figure II- 13 Drilling hardness and slope for Hole 9, Block 3

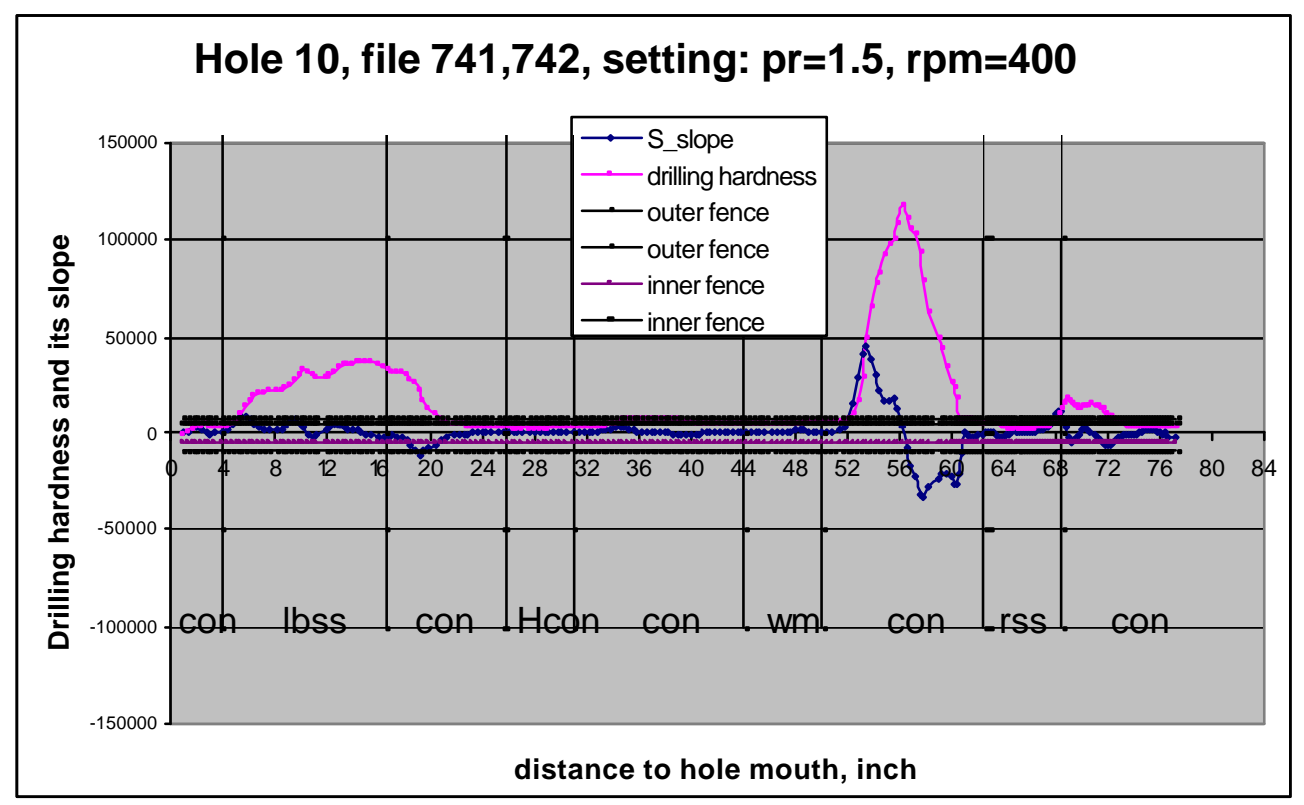

Figure II- 14 Drilling hardness and slope for Hole 10, Block 3 


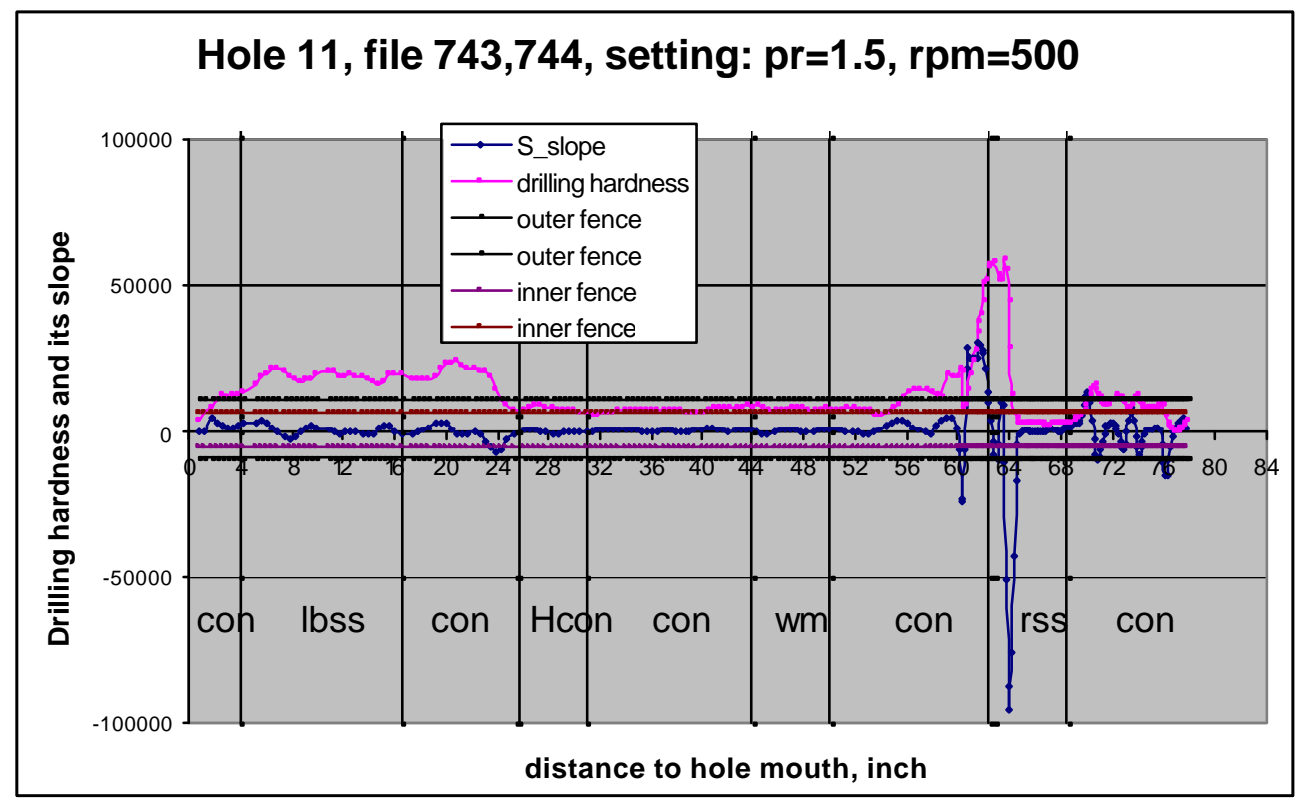

Figure II- 15 Drilling hardness and slope for Hole 11, Block 3 


\section{Appendix II}

Hole 23, file 794- 798, setting: $p r=0.6, r p m=300$

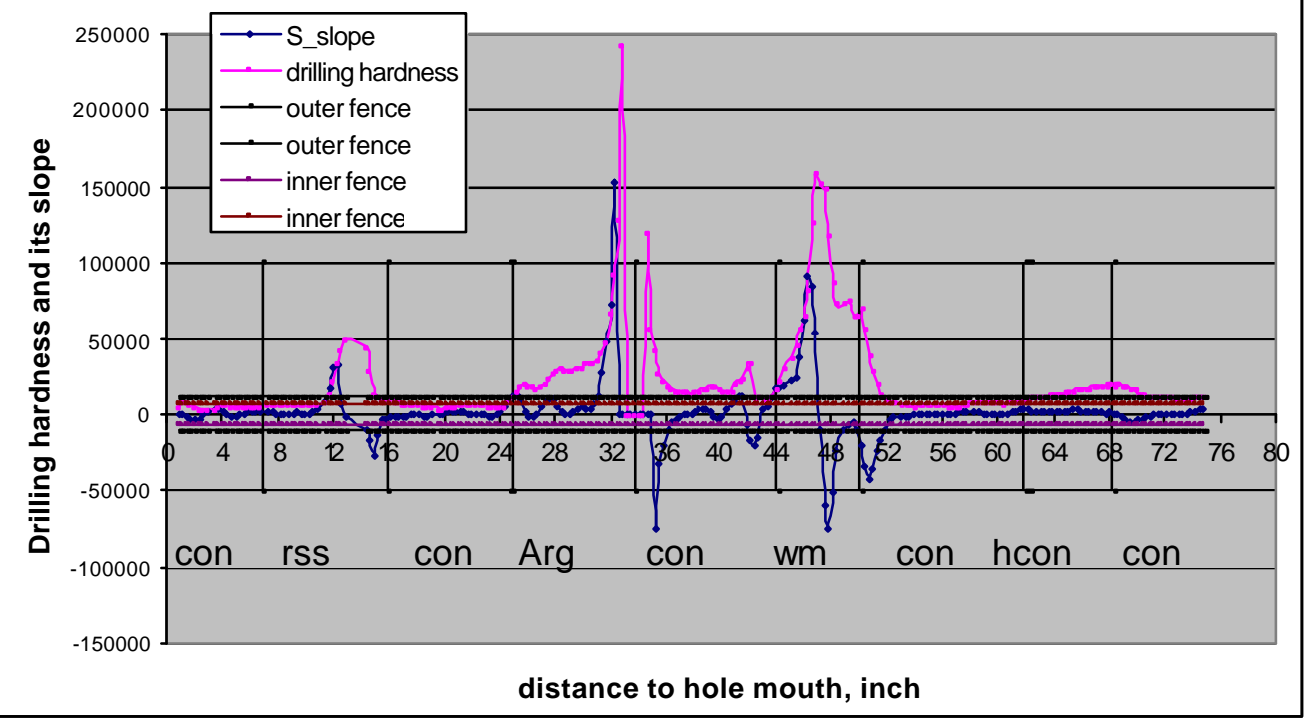

Figure II- 16 Drilling hardness and slope for Hole 23, Block 4

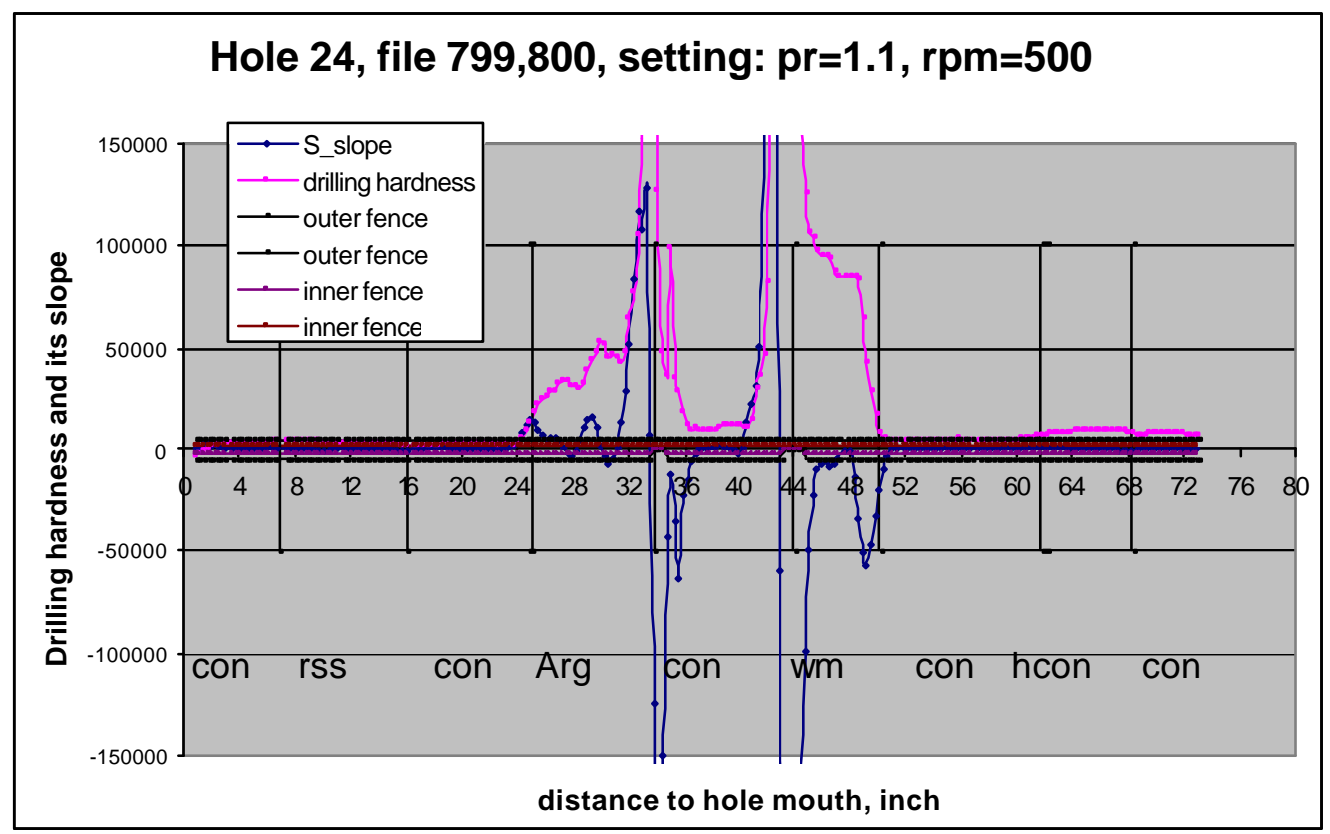

Figure II- 17 Drilling hardness and slope for Hole 24, Block 4 


\section{Appendix II}

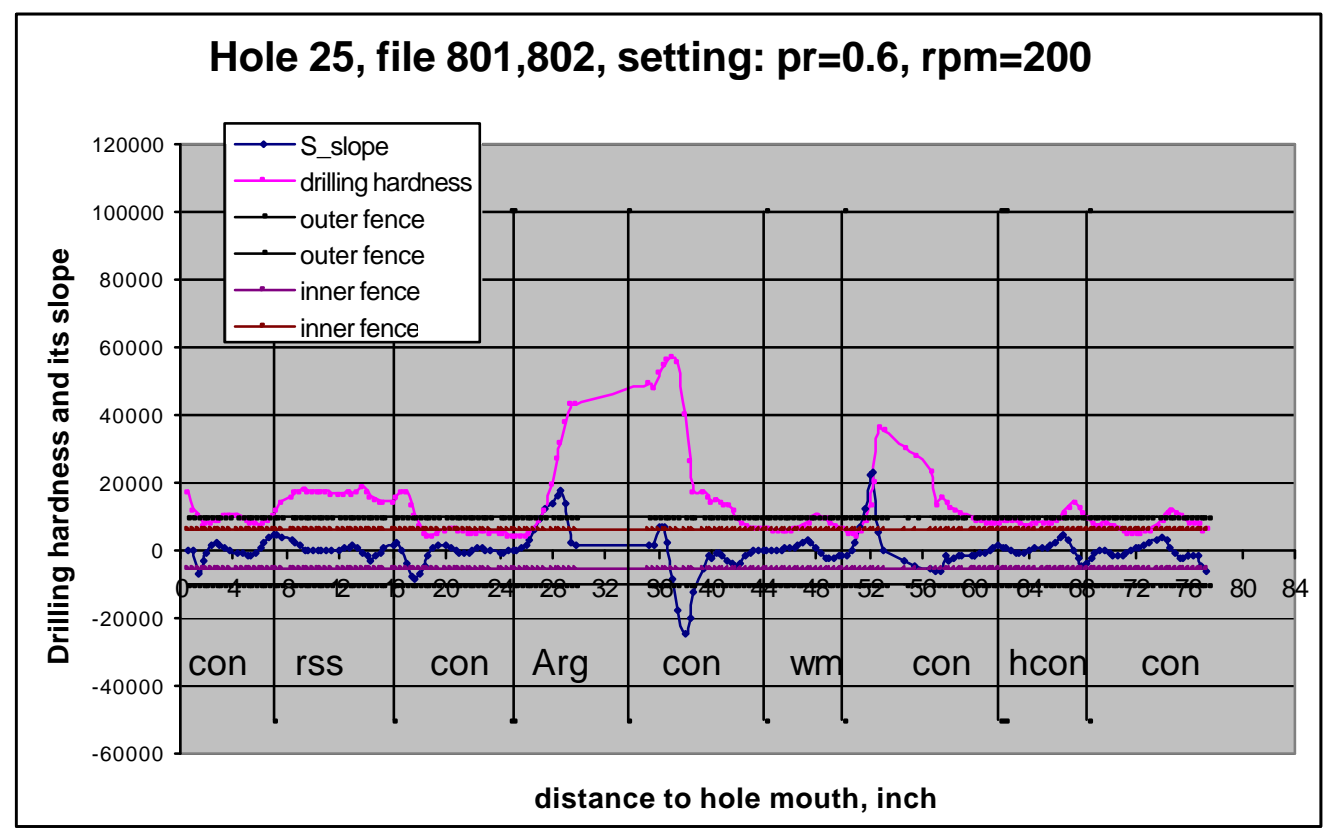

Figure II- 18 Drilling hardness and slope for Hole 25, Block 4

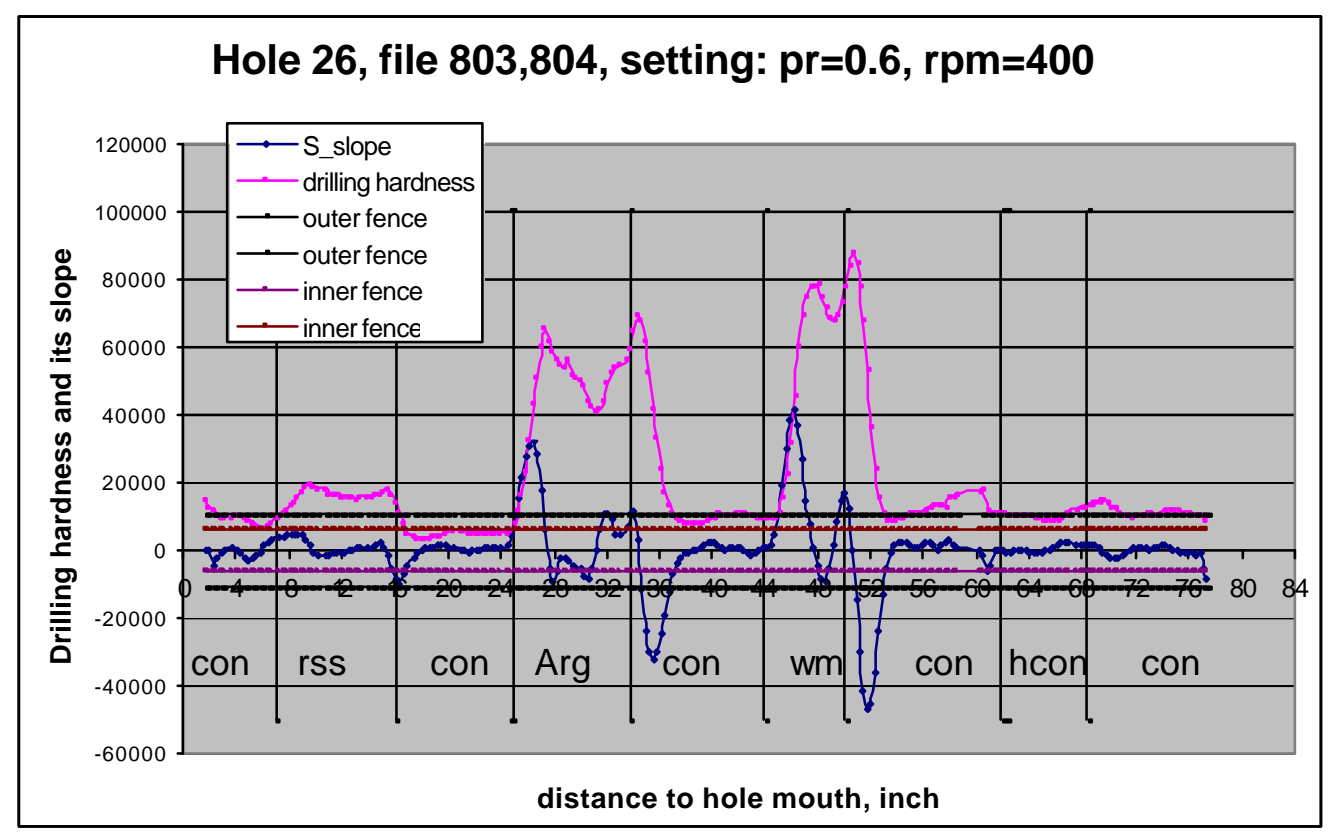

Figure II- 19 Drilling hardness and slope for Hole 26, Block 4 


\section{Appendix II}

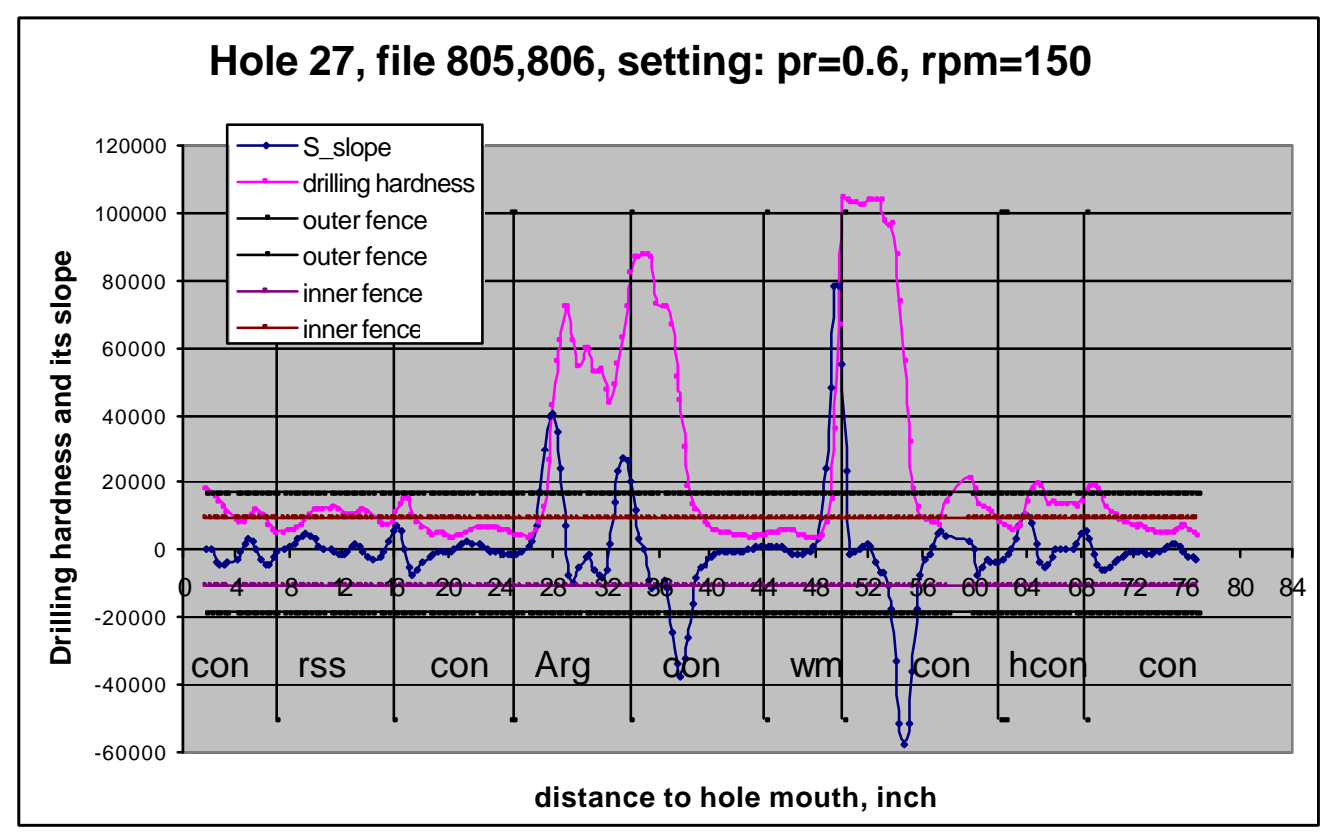

Figure II- 20 Drilling hardness and slope for Hole 27, Block 4

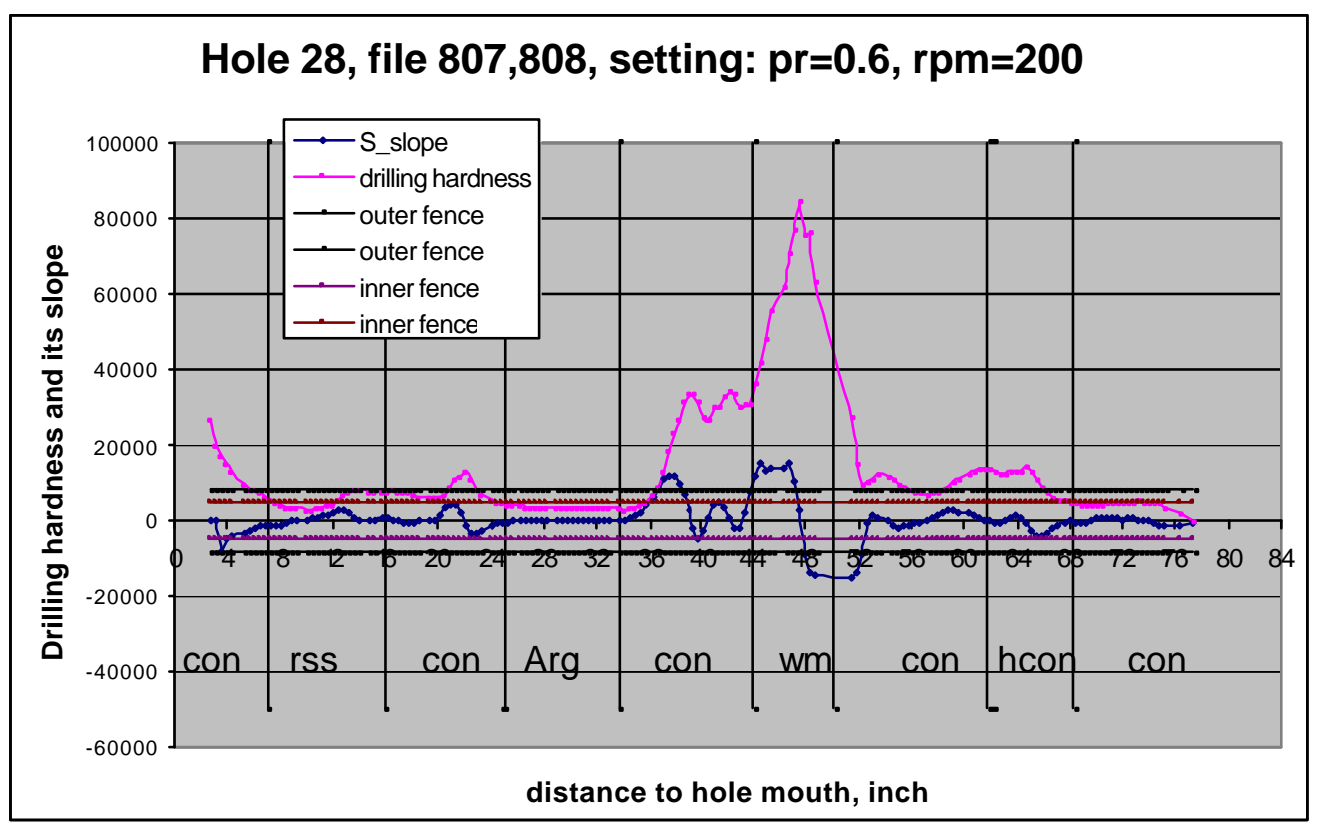

Figure II- 21 Drilling hardness and slope for Hole 28, Block 4 


\section{Appendix II}

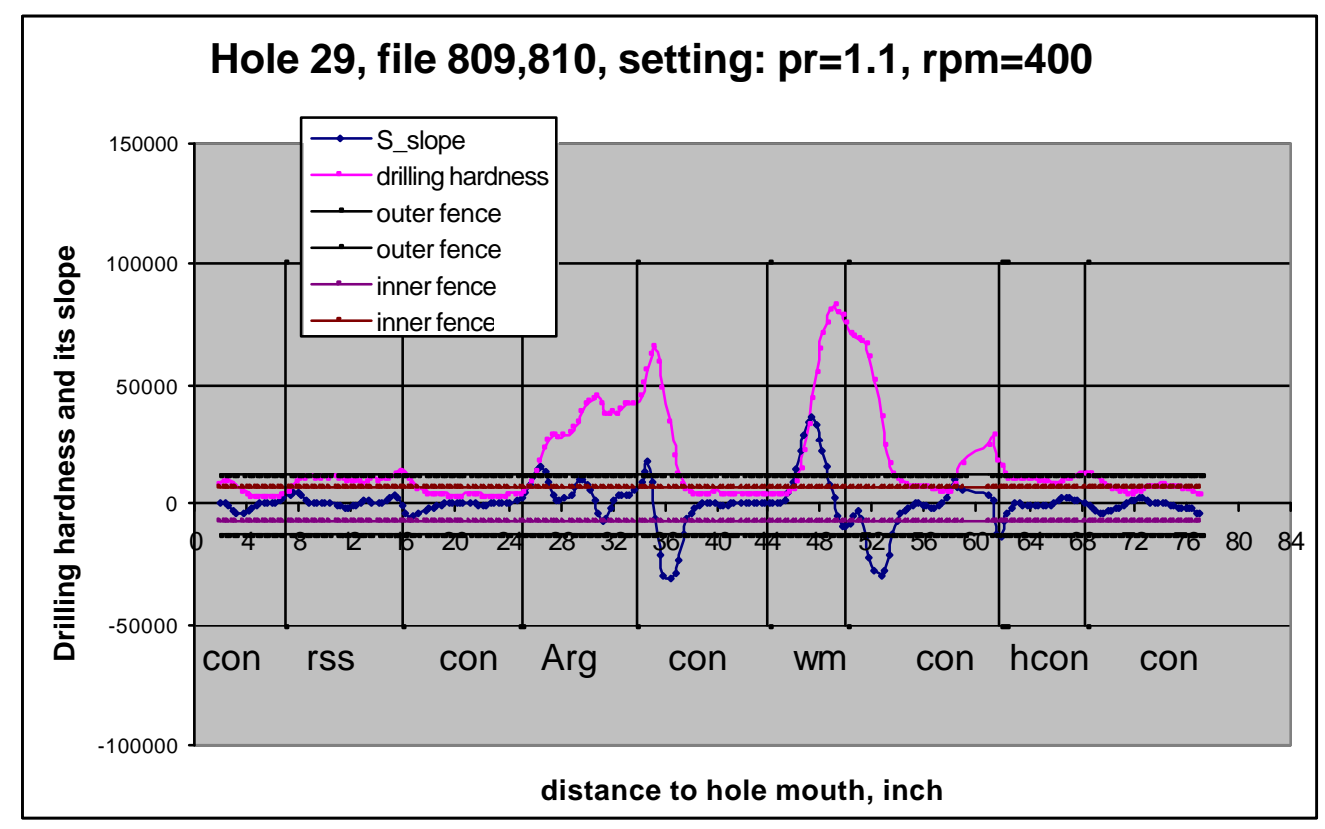

Figure II- 22 Drilling hardness and slope for Hole 29, Block 4

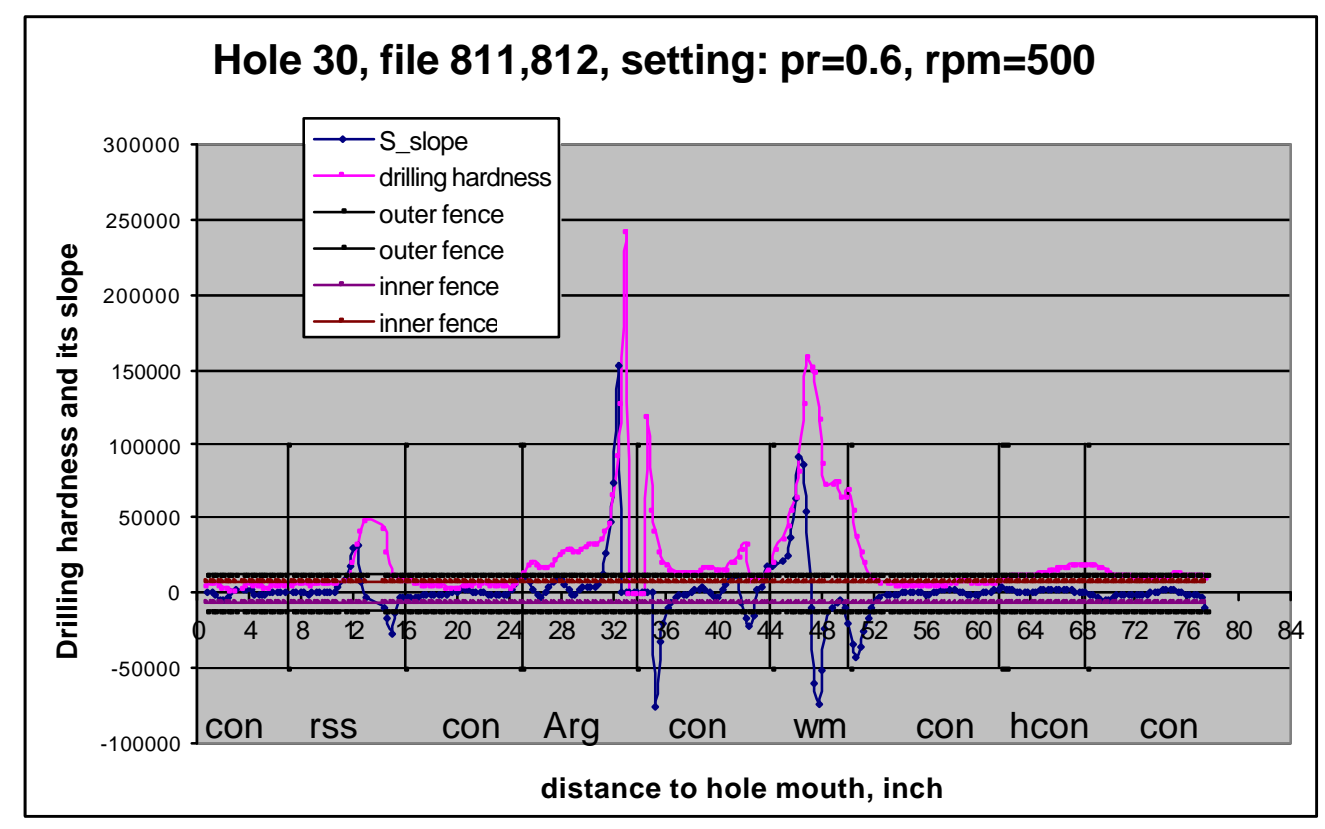

Figure II- 23 Drilling hardness and slope for Hole 30, Block 4 


\section{Appendix II}

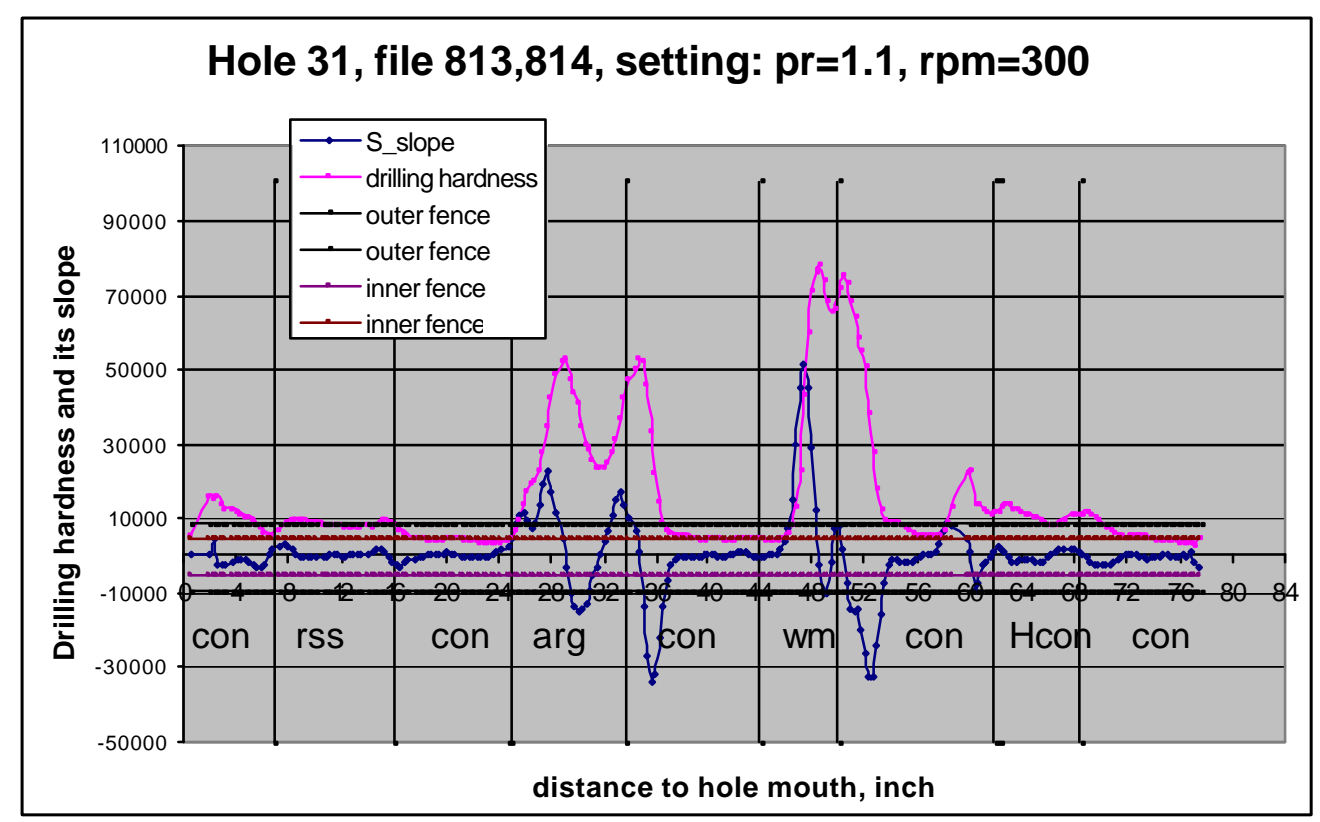

Figure II- 24 Drilling hardness and slope for Hole 31, Block 4

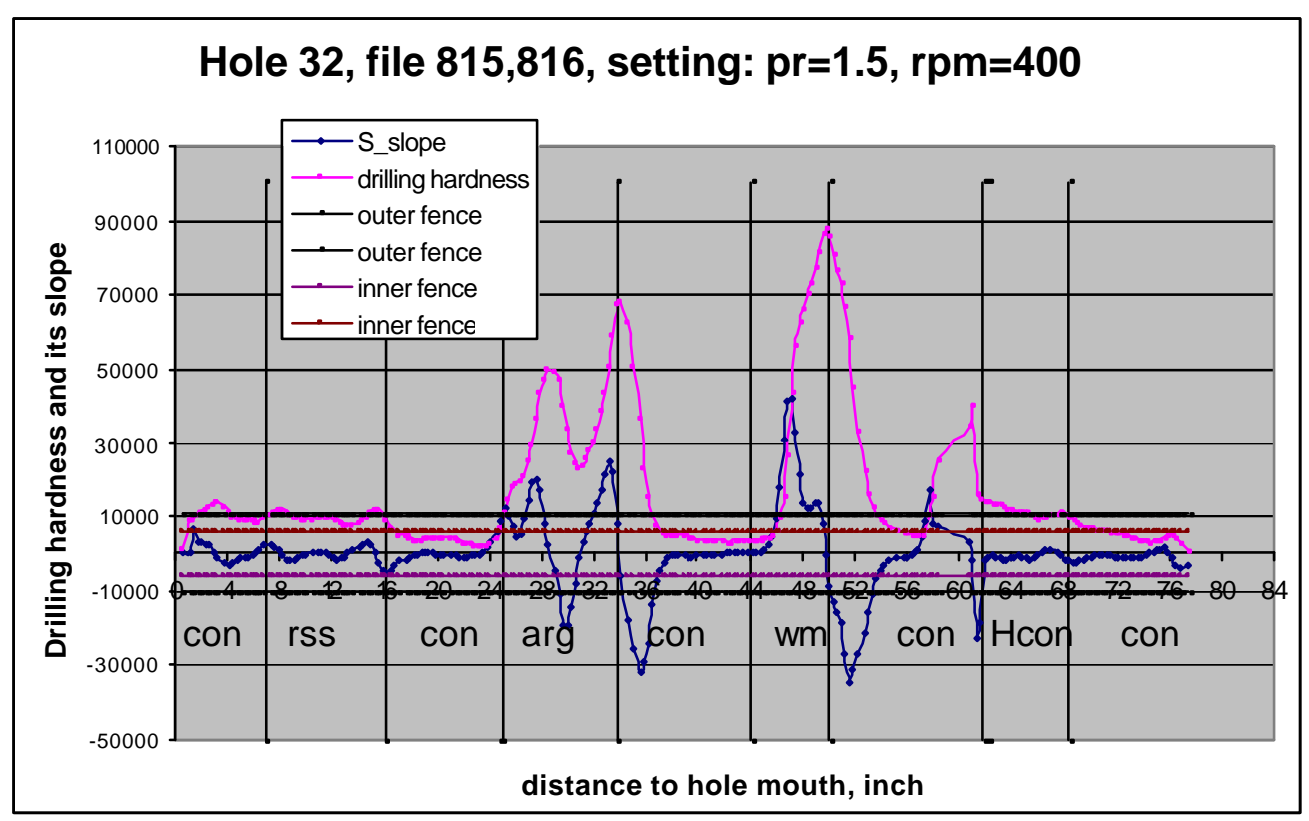

Figure II- 25 Drilling hardness and slope for Hole 32, Block 4 


\section{Appendix II}

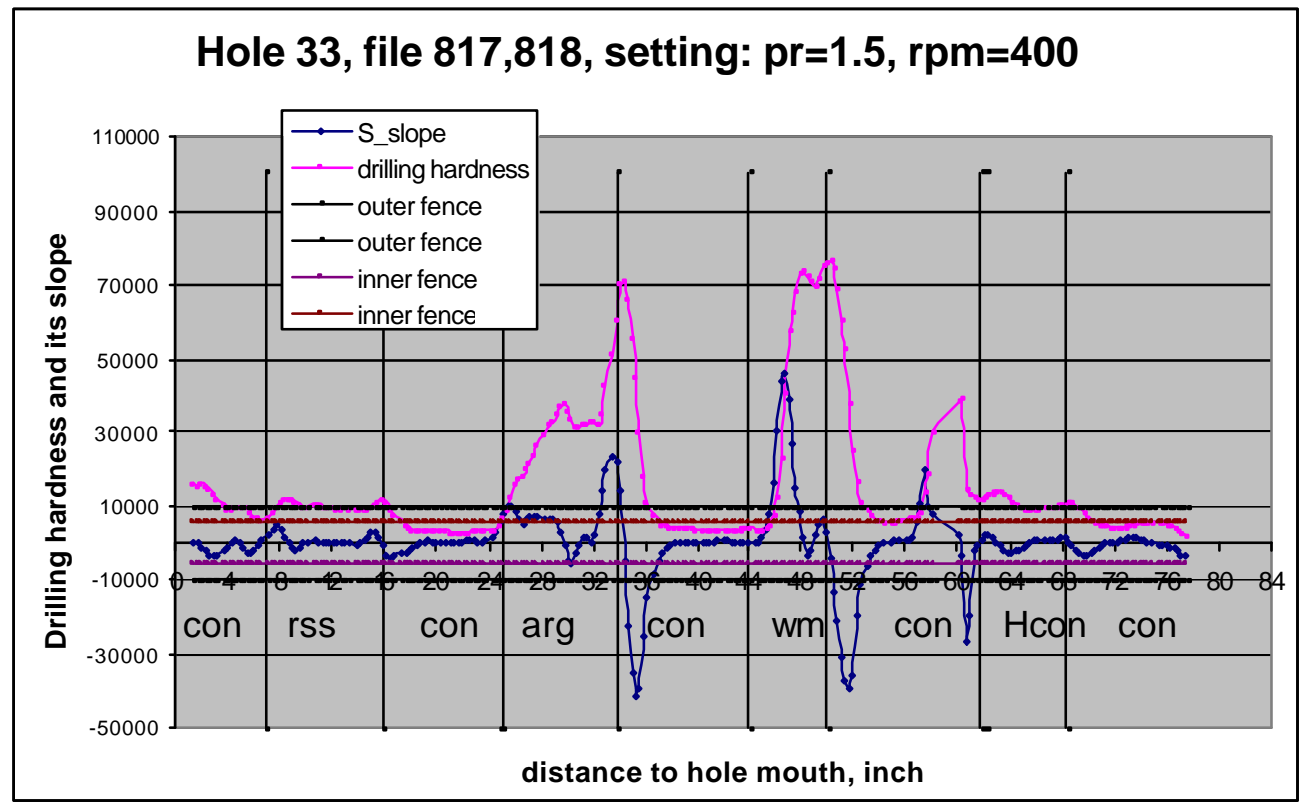

Figure II- 26 Drilling hardness and slope for Hole 33, Block 4 


\section{$\underline{\text { Appendix II }}$}

Fractured Concrete Block, Figure II- 27 to Figure II- 33.

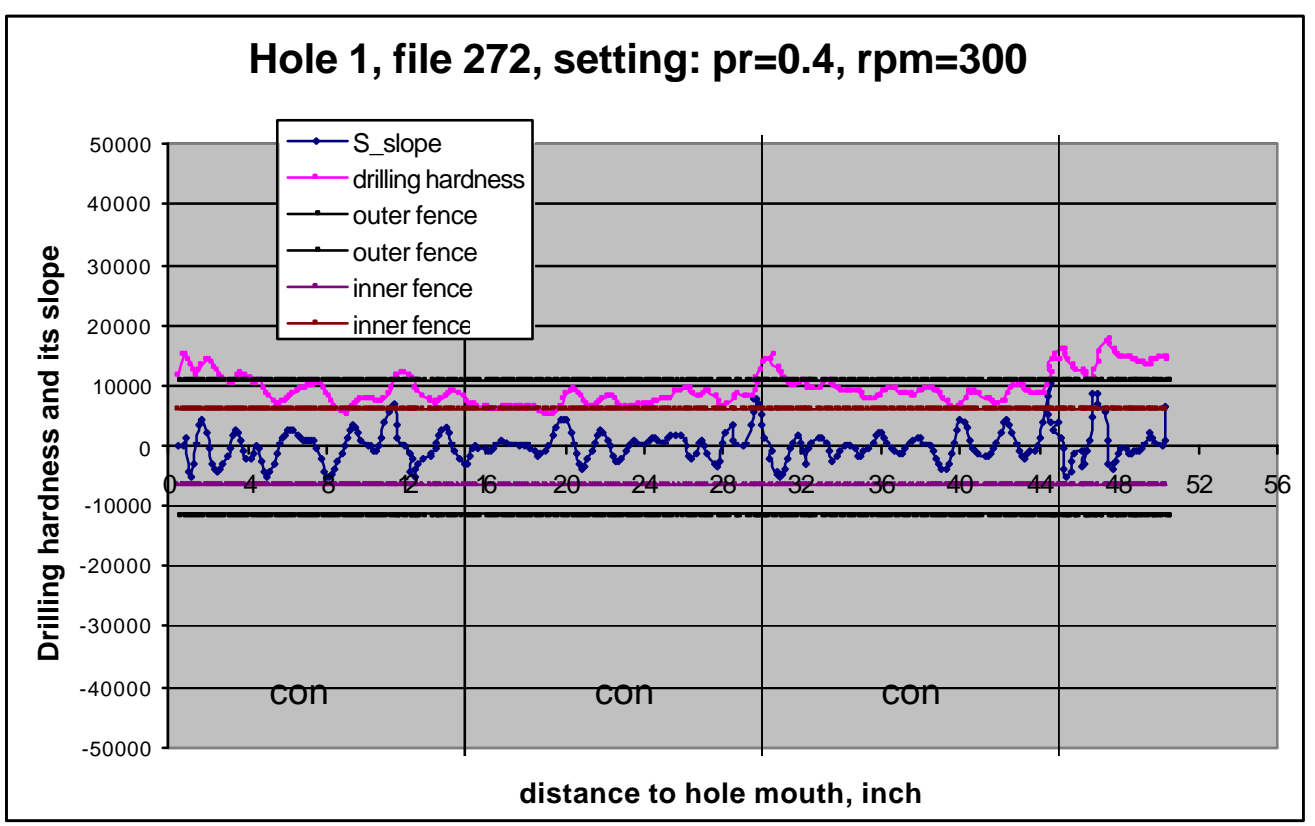

Figure II- 27 Drilling hardness and slope for Hole 1, Fractured Block

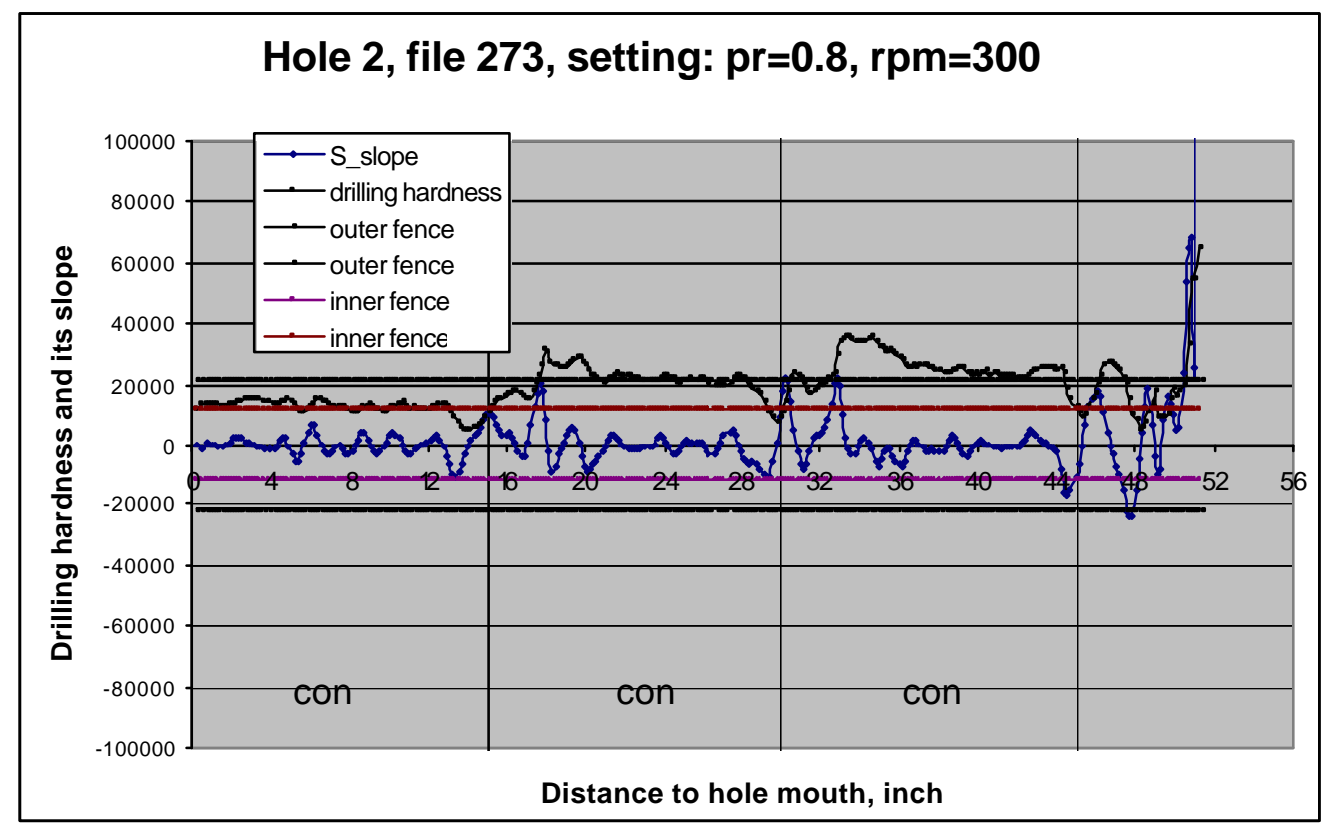

Figure II- 28 Drilling hardness and slope for Hole 2, Fractured Block 


\section{Appendix II}

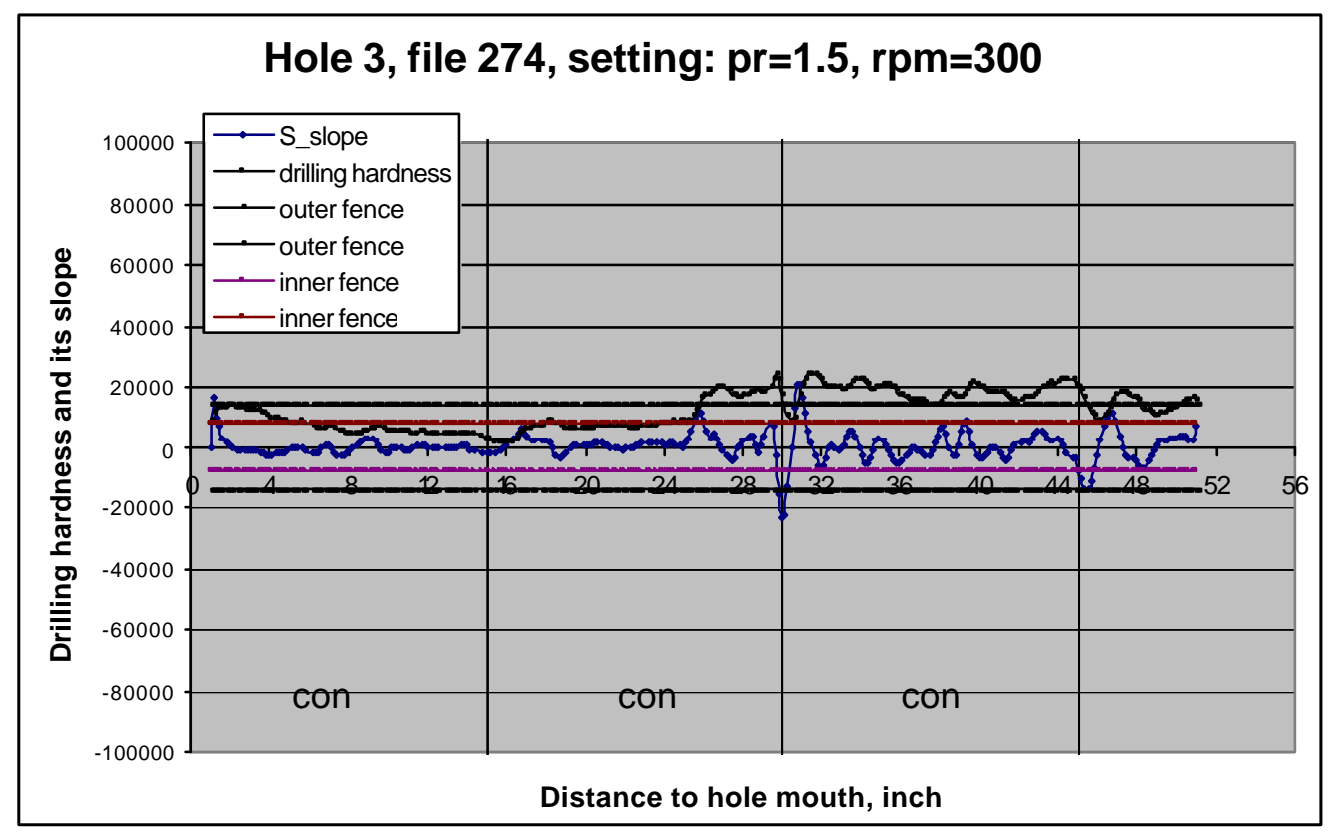

Figure II- 29 Drilling hardness and slope for Hole 3, Fractured Block

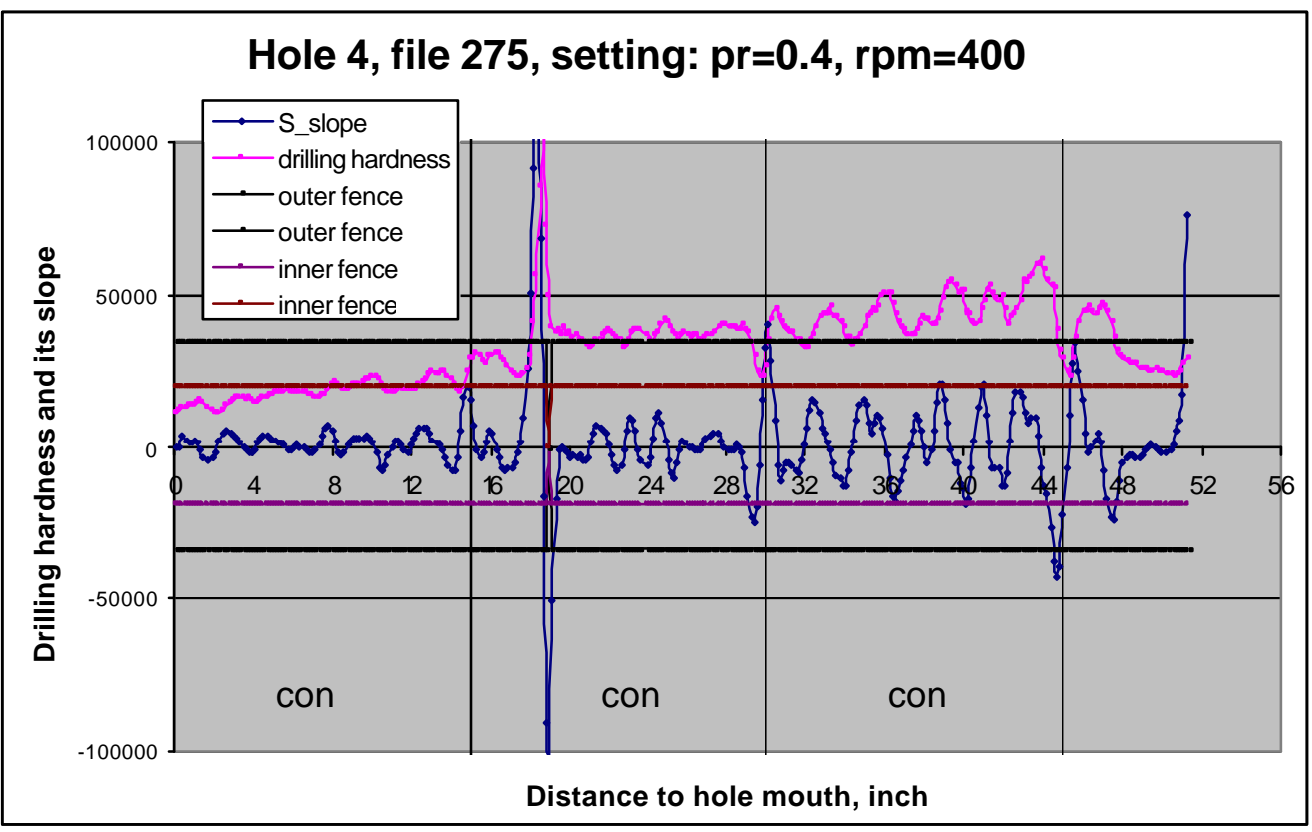

Figure II- 30 Drilling hardness and slope for Hole 4, Fractured Block 


\section{$\underline{\text { Appendix II }}$}

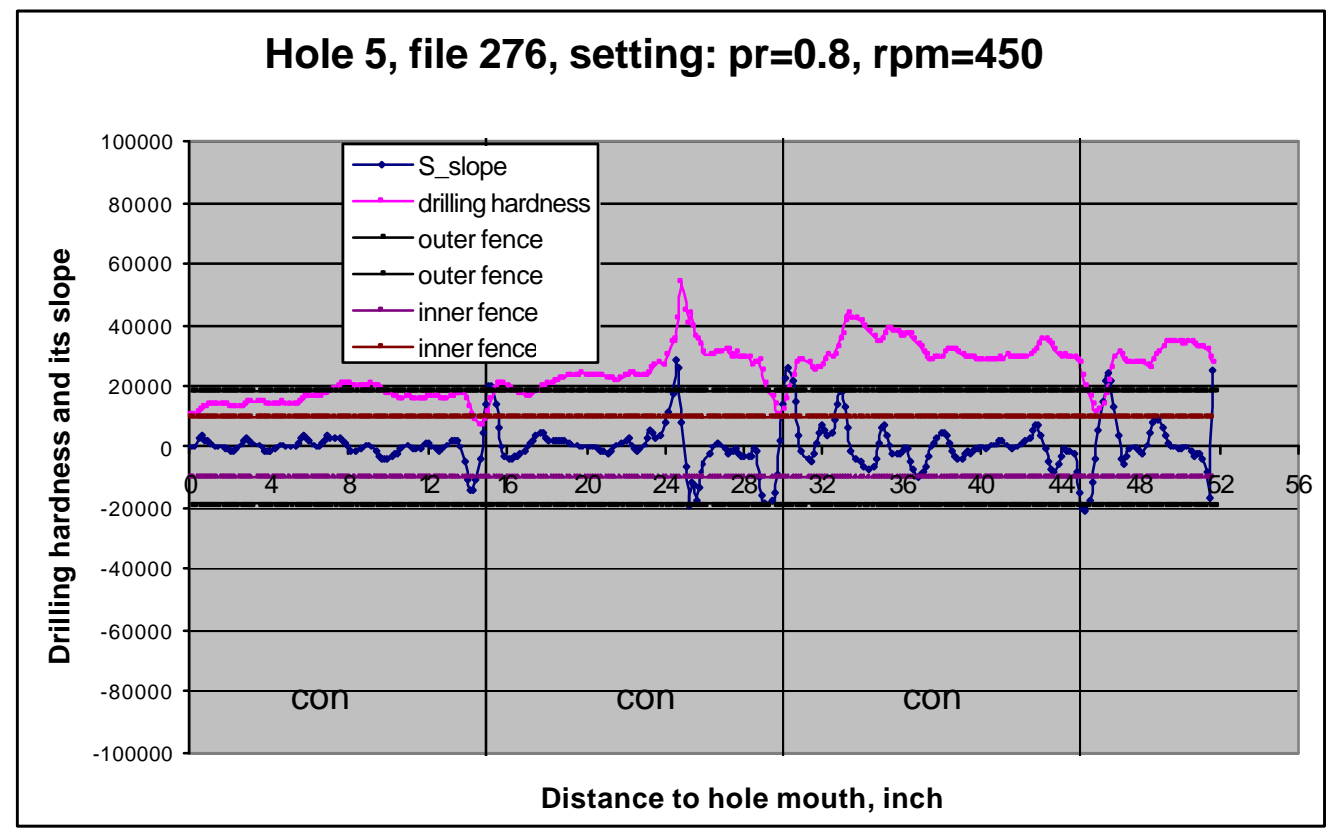

Figure II- 31 Drilling hardness and slope for Hole 5, Fractured Block

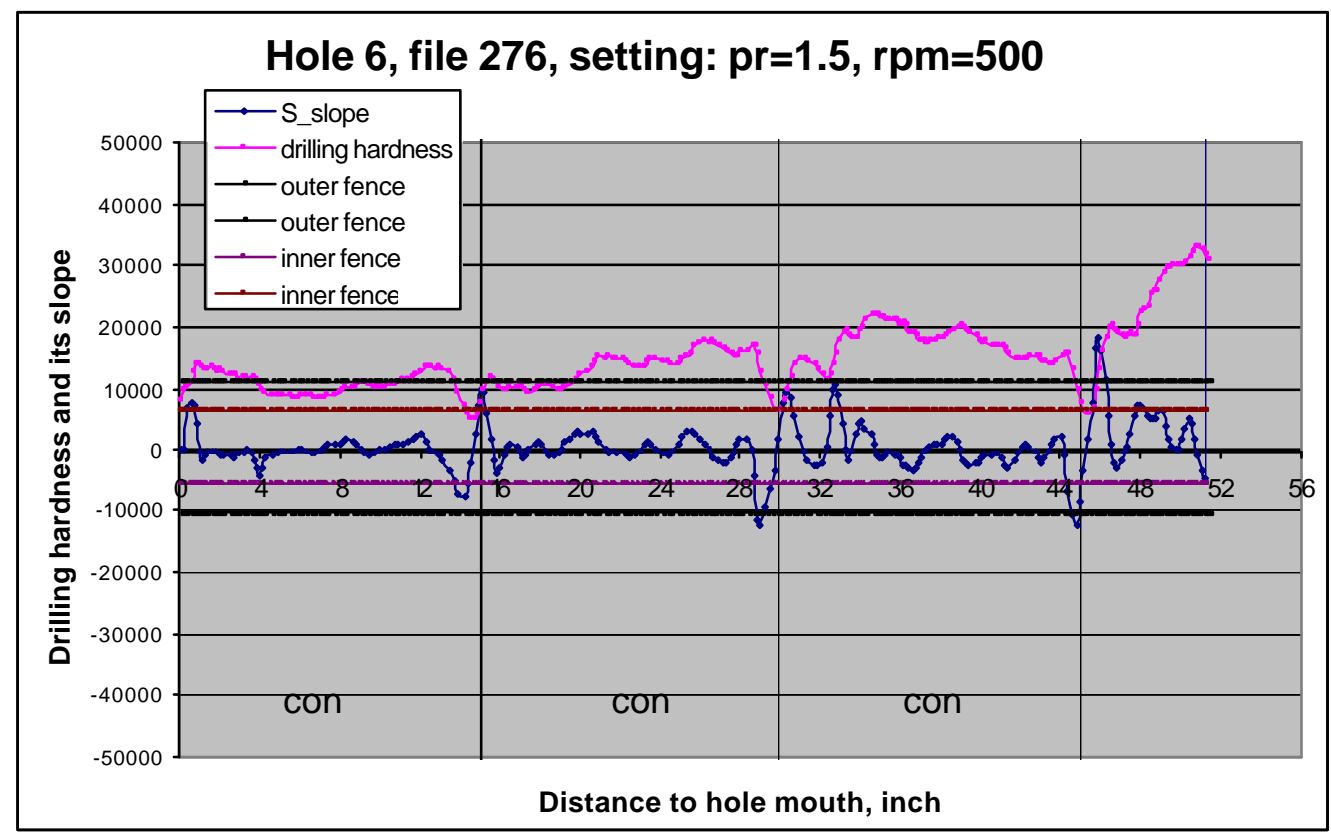

Figure II- 32 Drilling hardness and slope for Hole 6, Fractured Block 


\section{Appendix II}

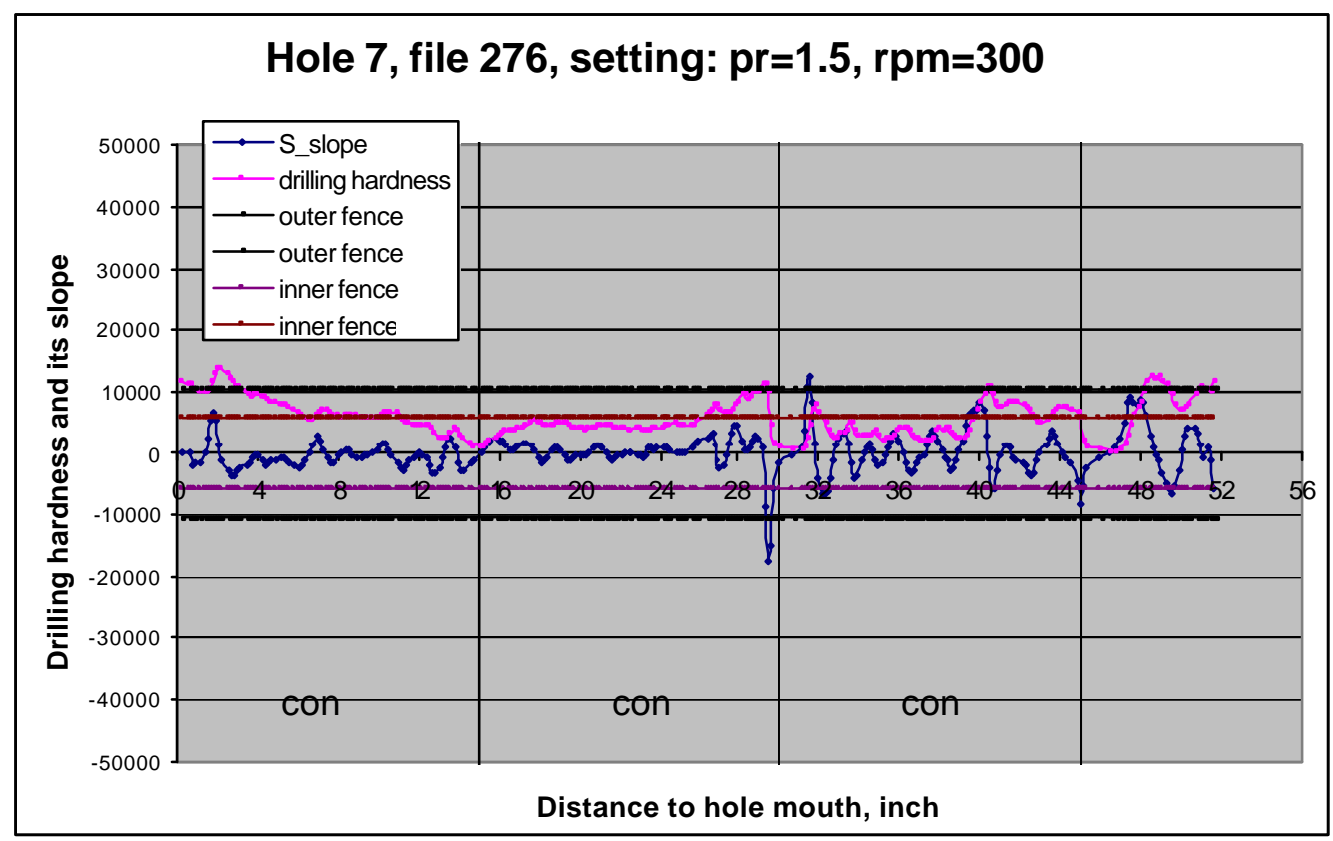

Figure II- 33 Drilling hardness and slope for Hole 7, Fractured Block 


\section{Appendix II}

Solid Block, Figure II- 34 to Figure II- 45.

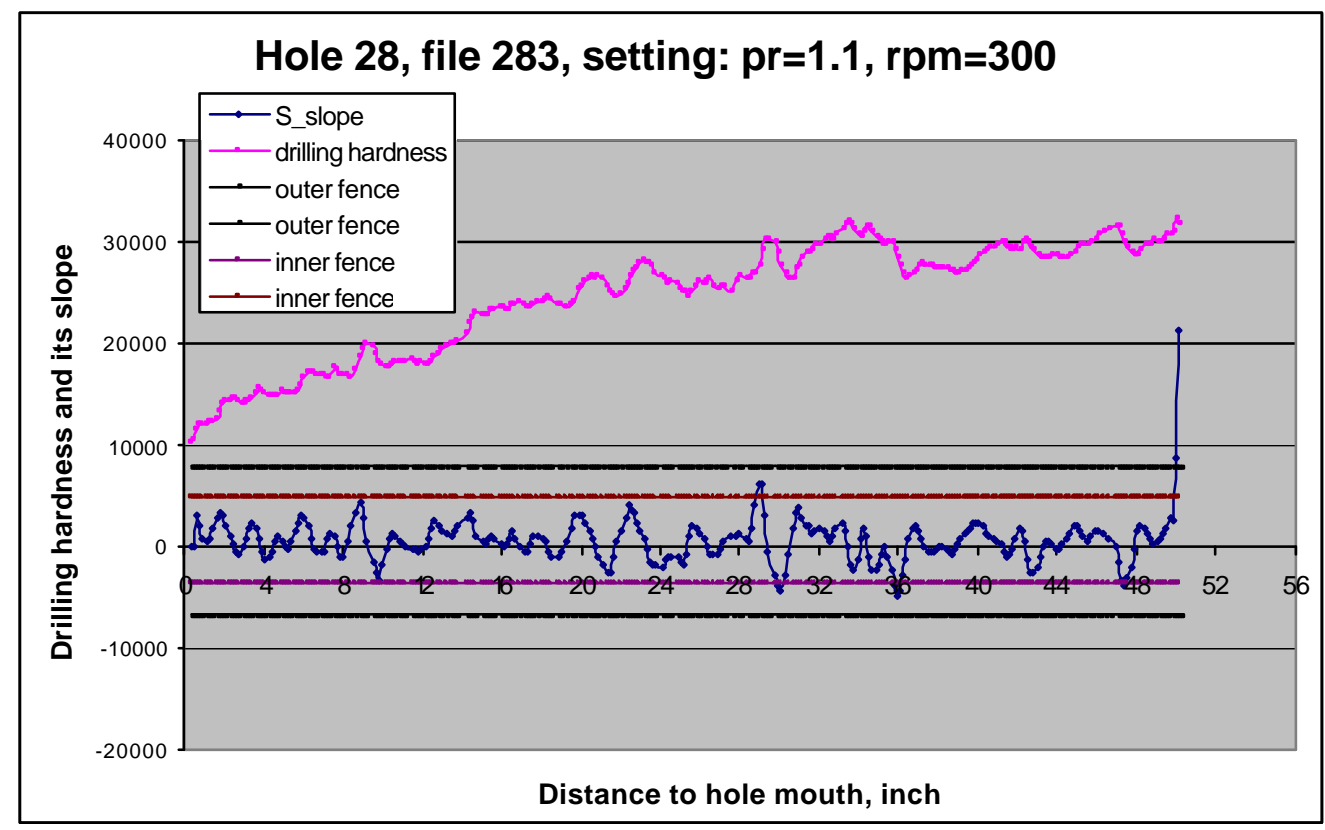

Figure II- 34 Drilling hardness and slope for Hole 28, Solid Block

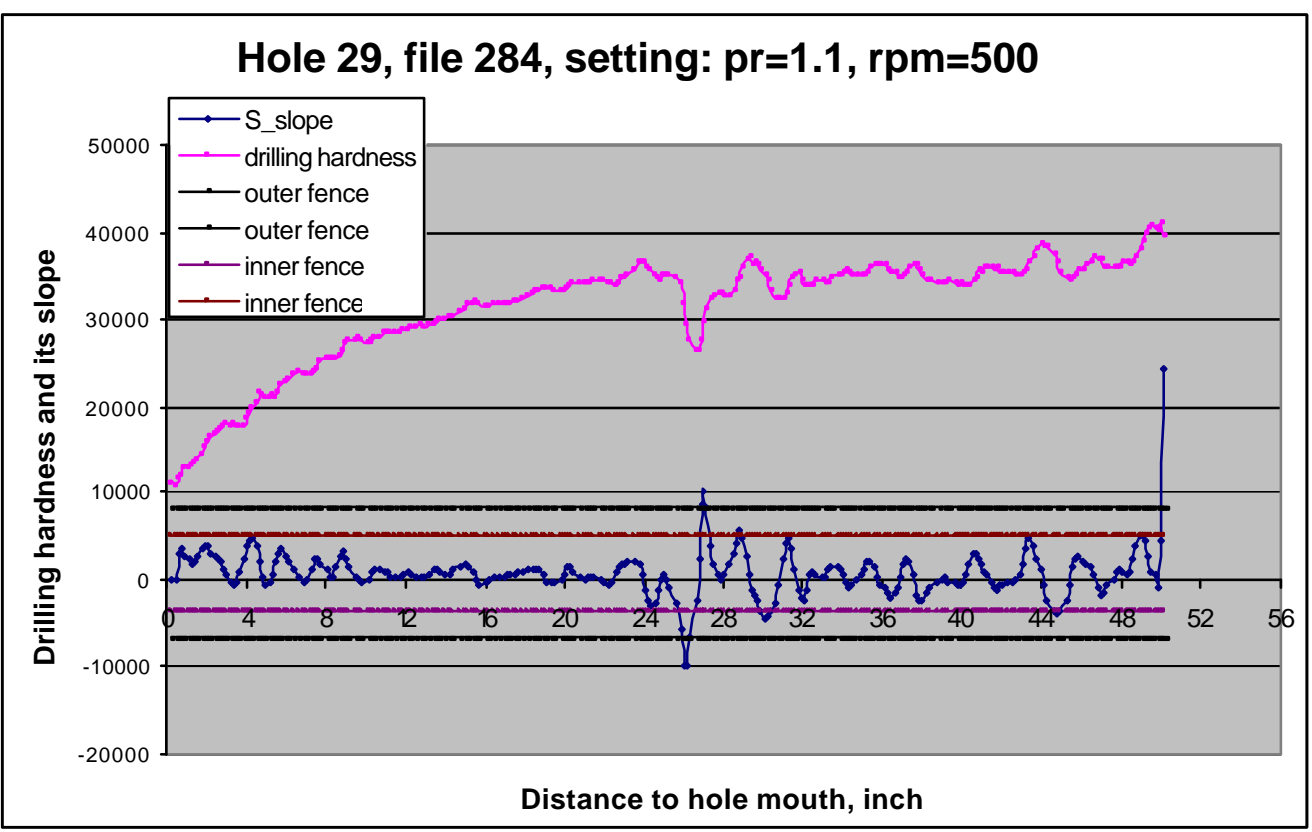

Figure II- 35 Drilling hardness and slope for Hole 29, Solid Block 


\section{$\underline{\text { Appendix II }}$}

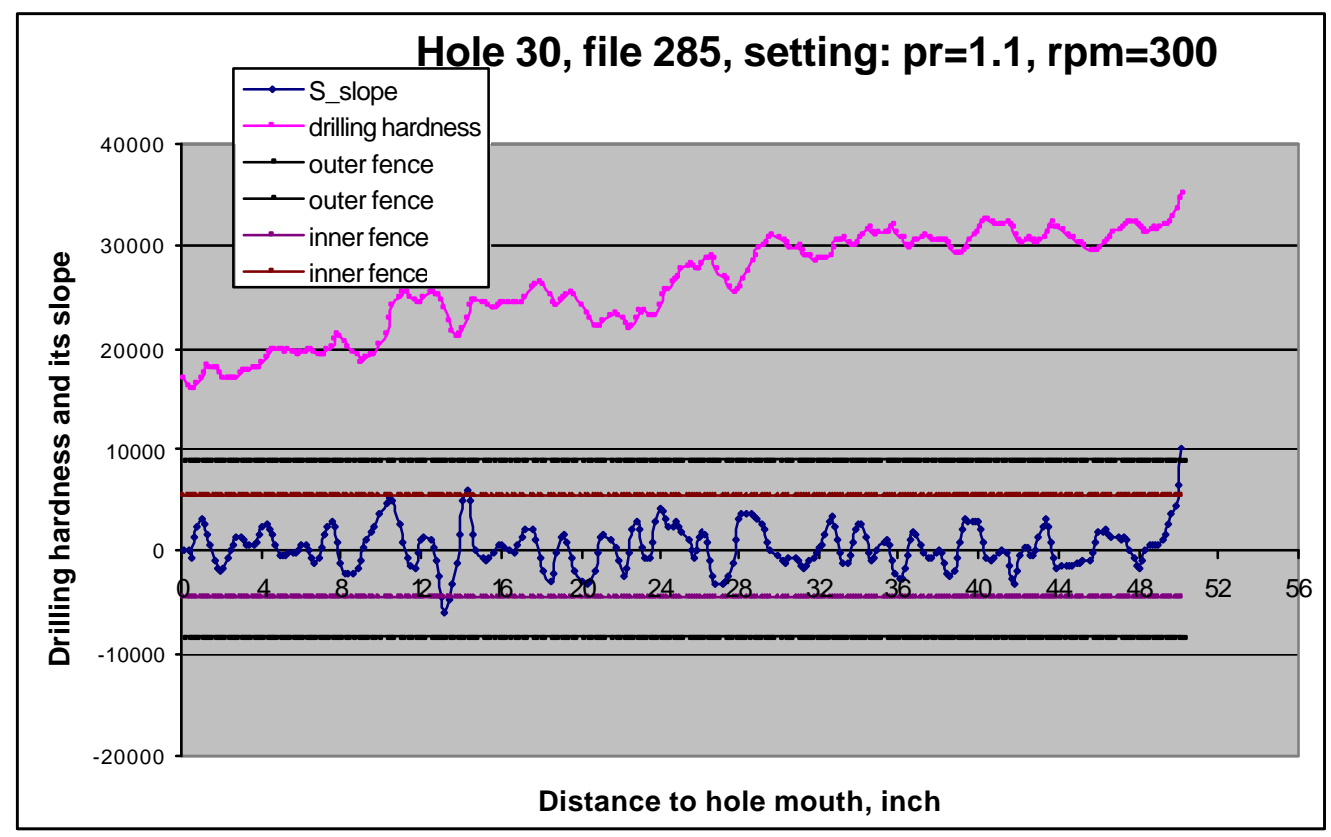

Figure II- 36 Drilling hardness and slope for Hole 30, Solid Block

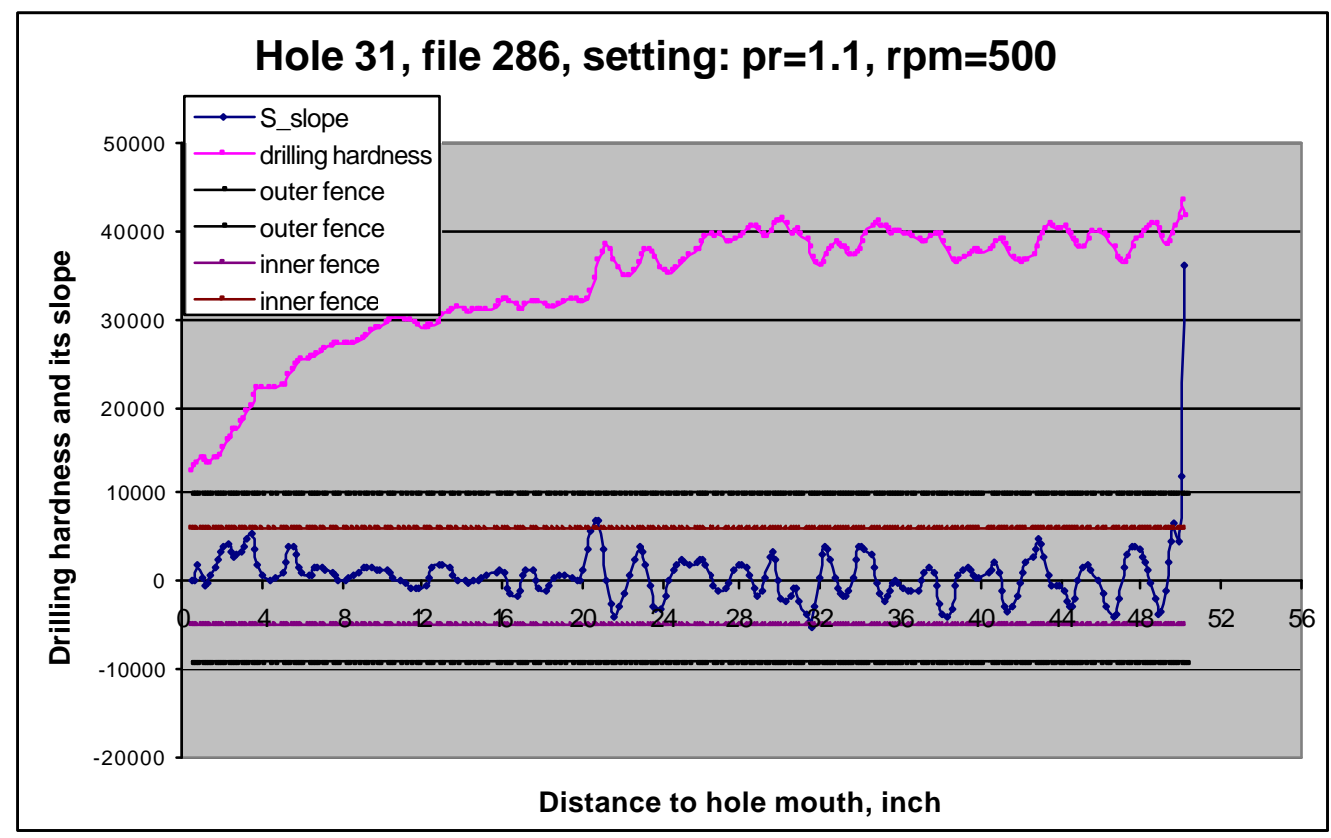

Figure II- 37 Drilling hardness and slope for Hole 31, Solid Block 


\section{Appendix II}

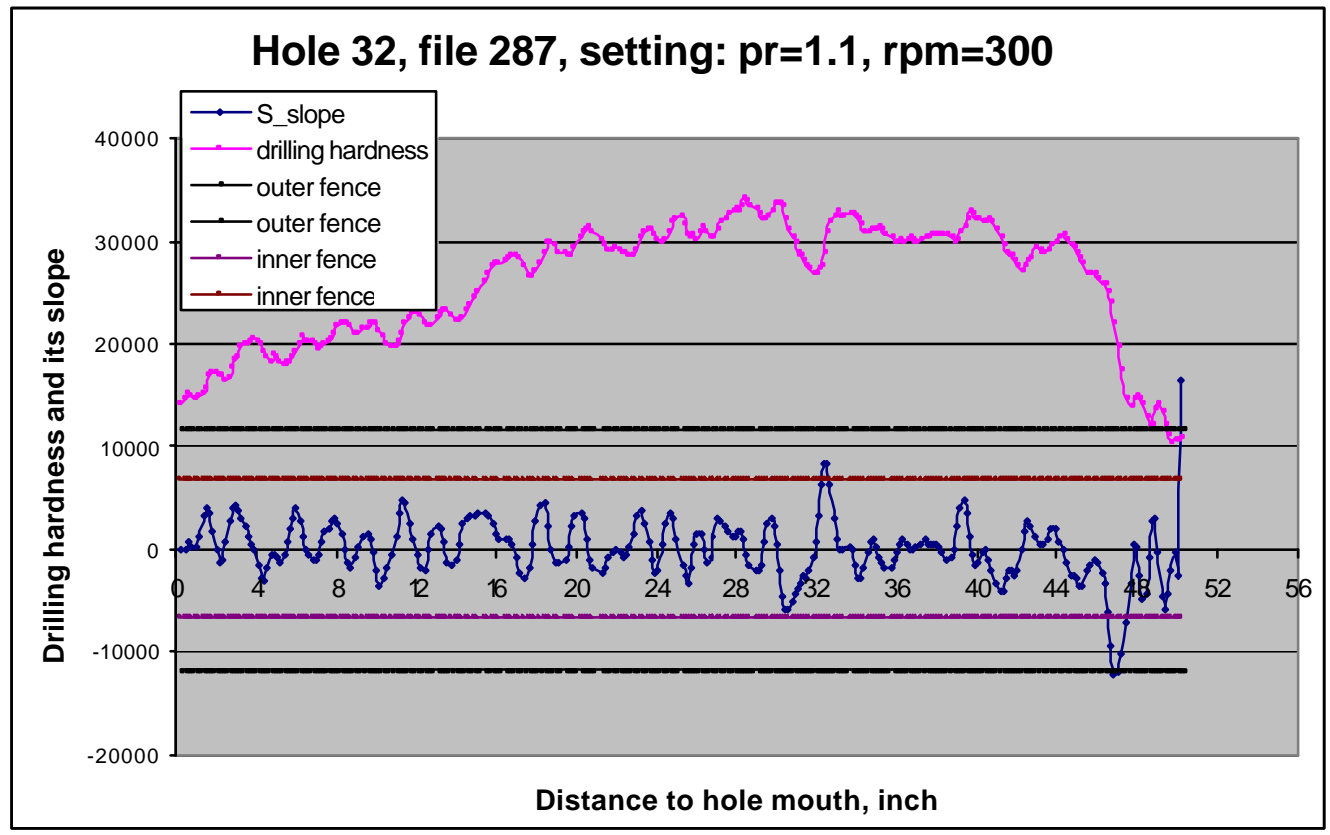

Figure II- 38 Drilling hardness and slope for Hole 32, Solid Block

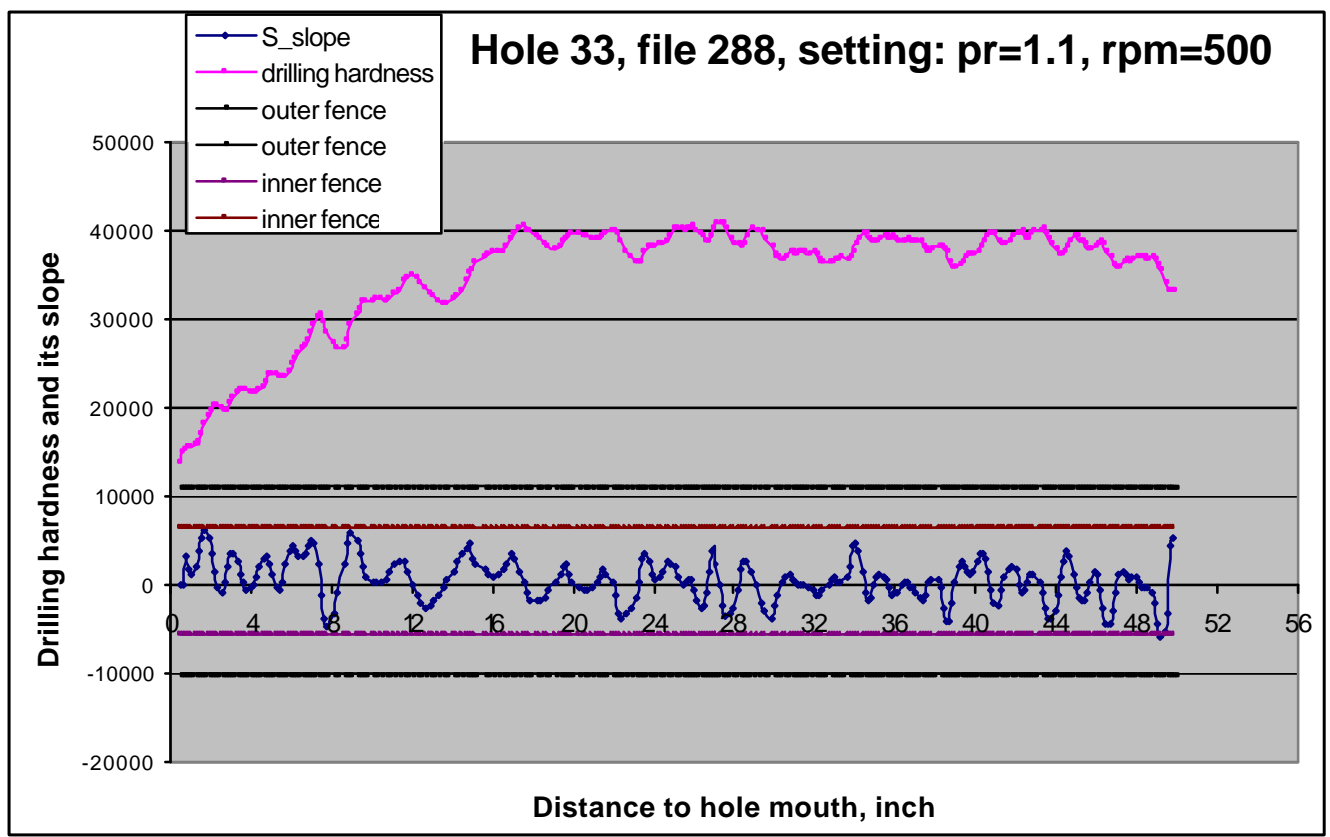

Figure II- 39 Drilling hardness and slope for Hole 33, Solid Block 


\section{Appendix II}

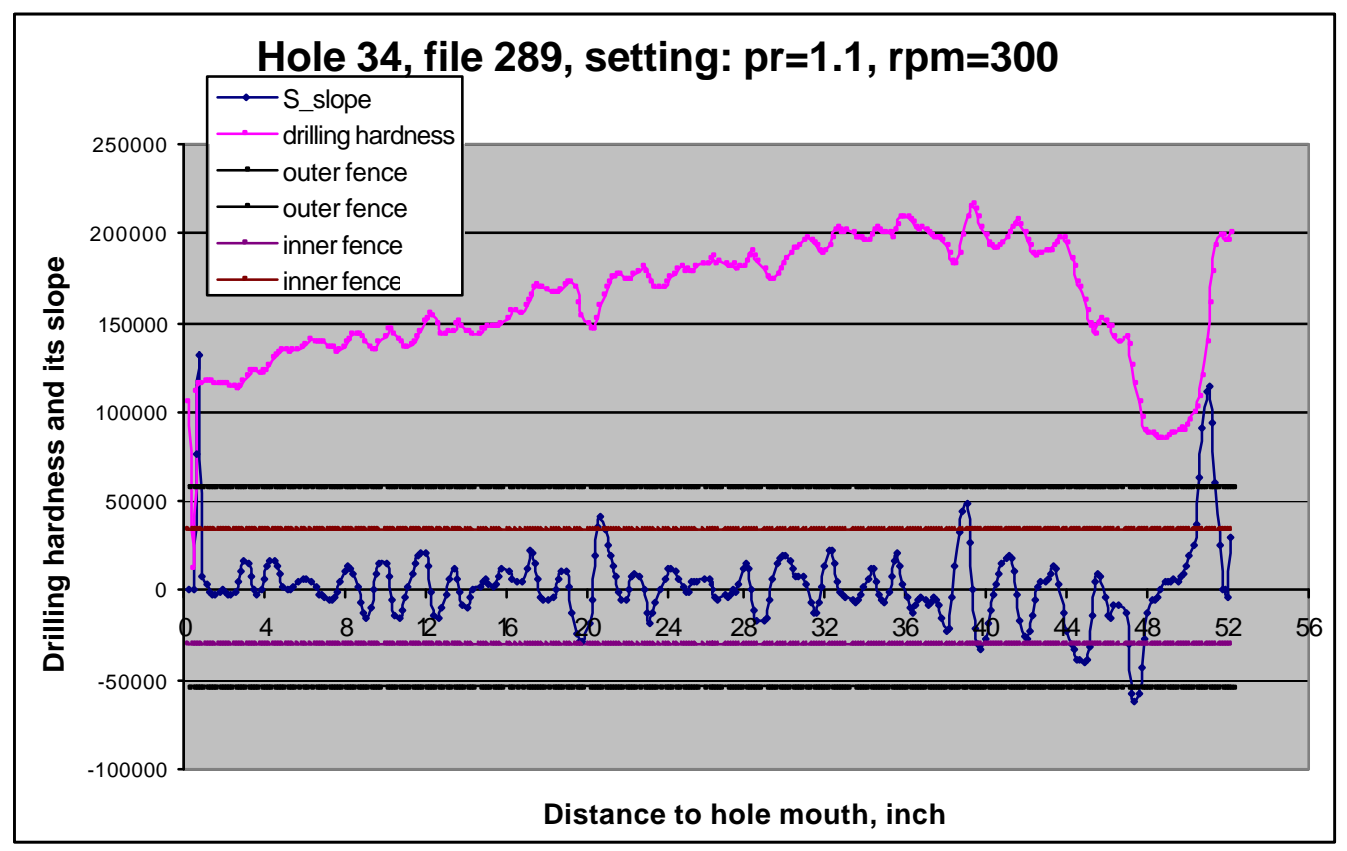

Figure II- 40 Drilling hardness and slope for Hole 34, Solid Block

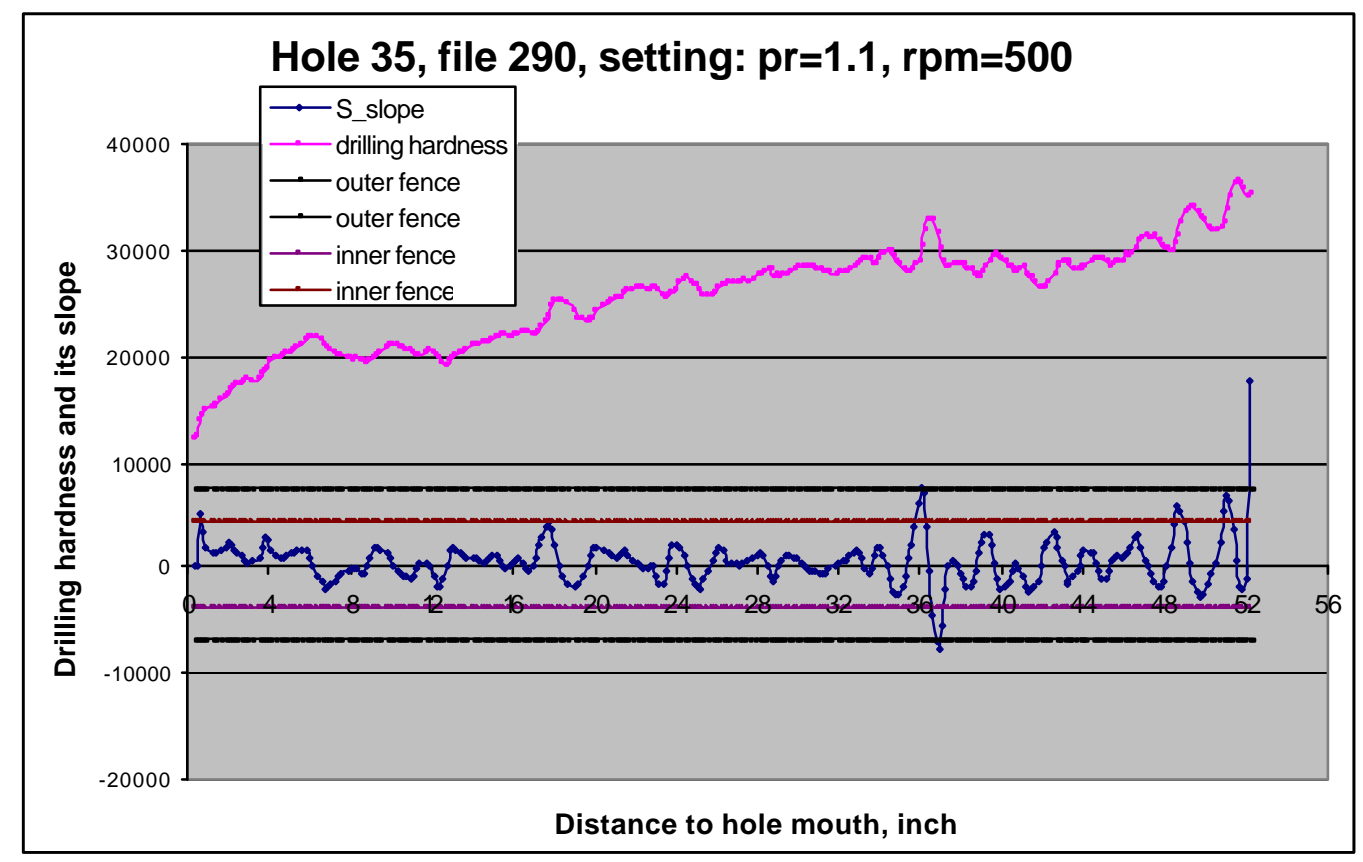

Figure II- 41 Drilling hardness and slope for Hole 35, Solid Block 


\section{Appendix II}

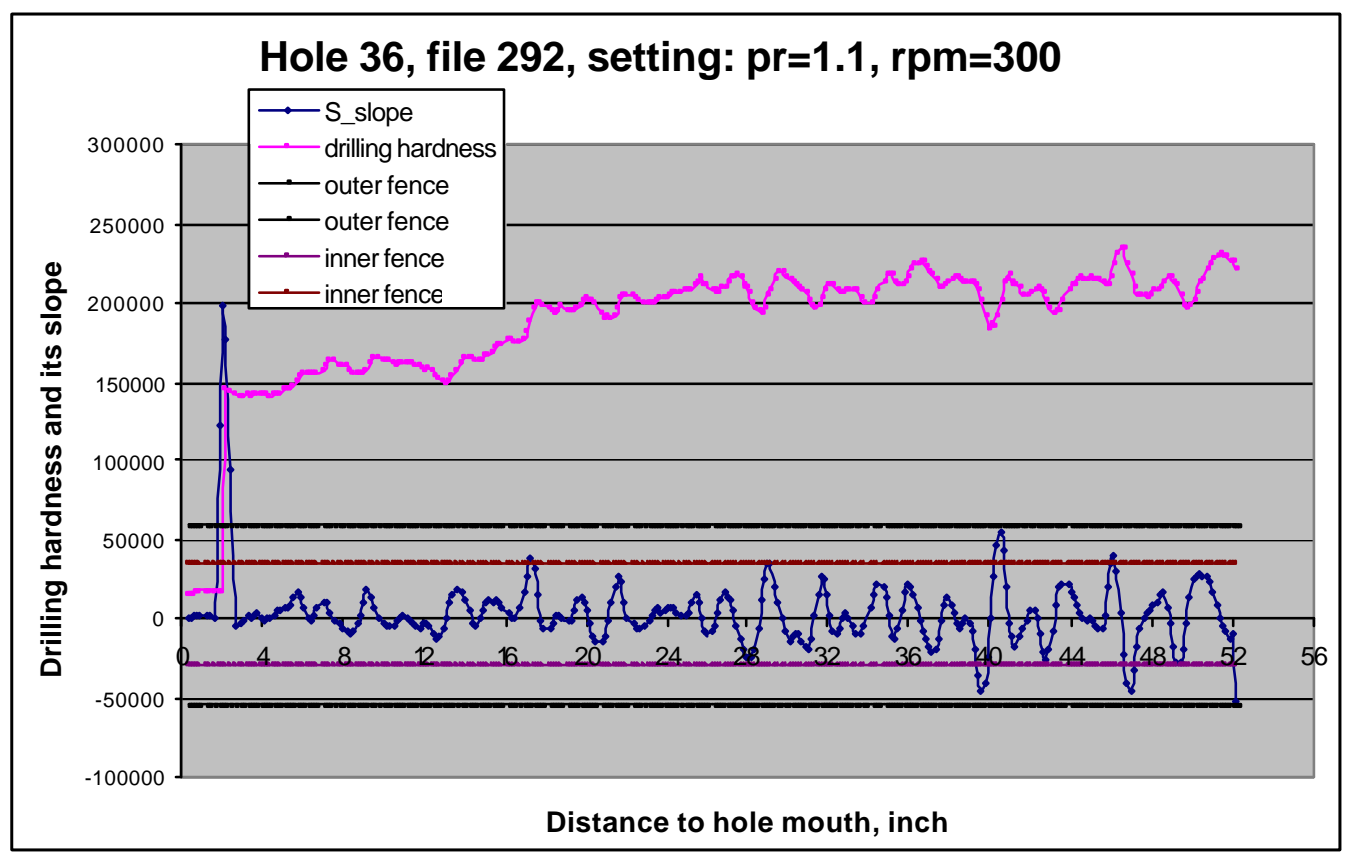

Figure II- 42 Drilling hardness and slope for Hole 36, Solid Block

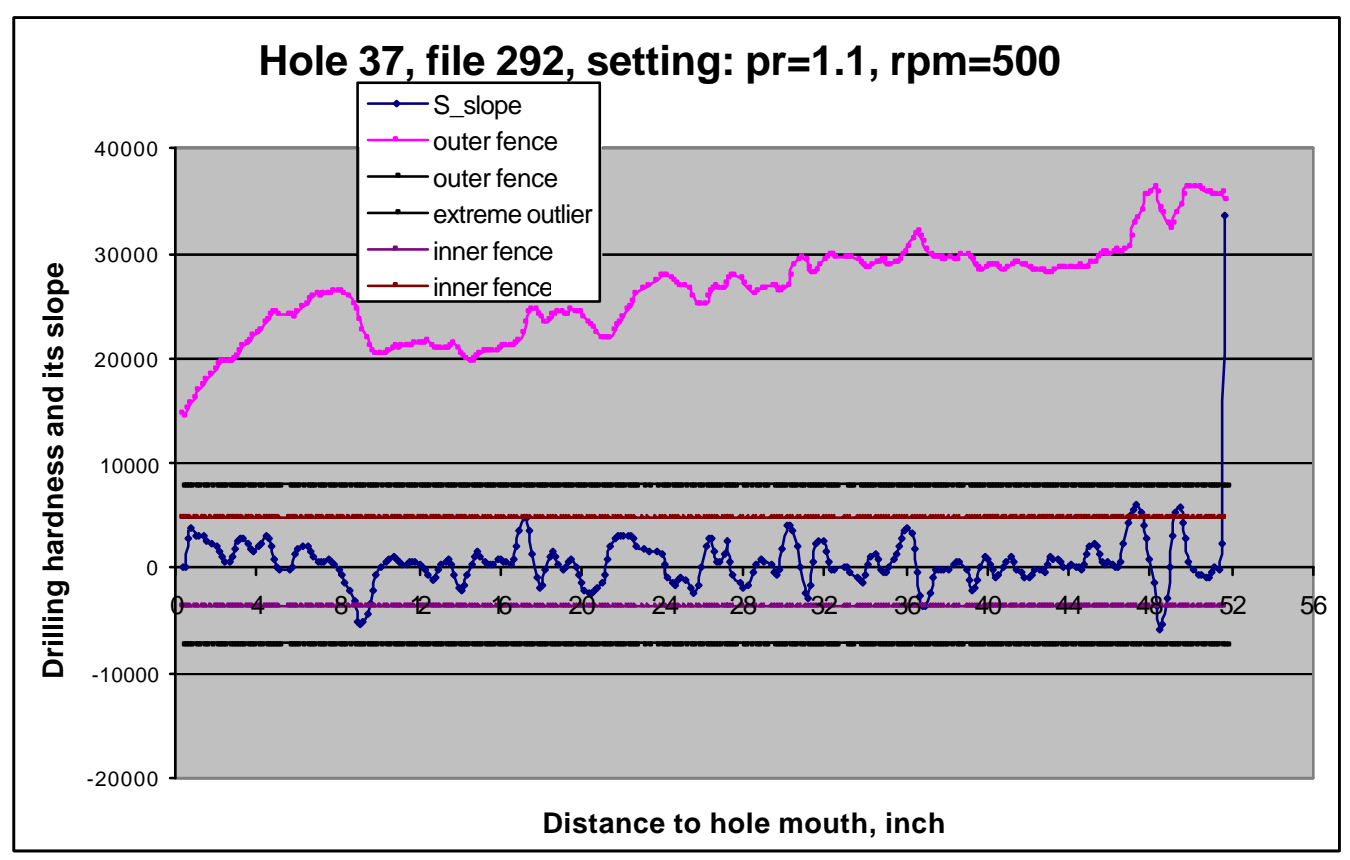

Figure II- 43 Drilling hardness and slope for Hole 37, Solid Block 


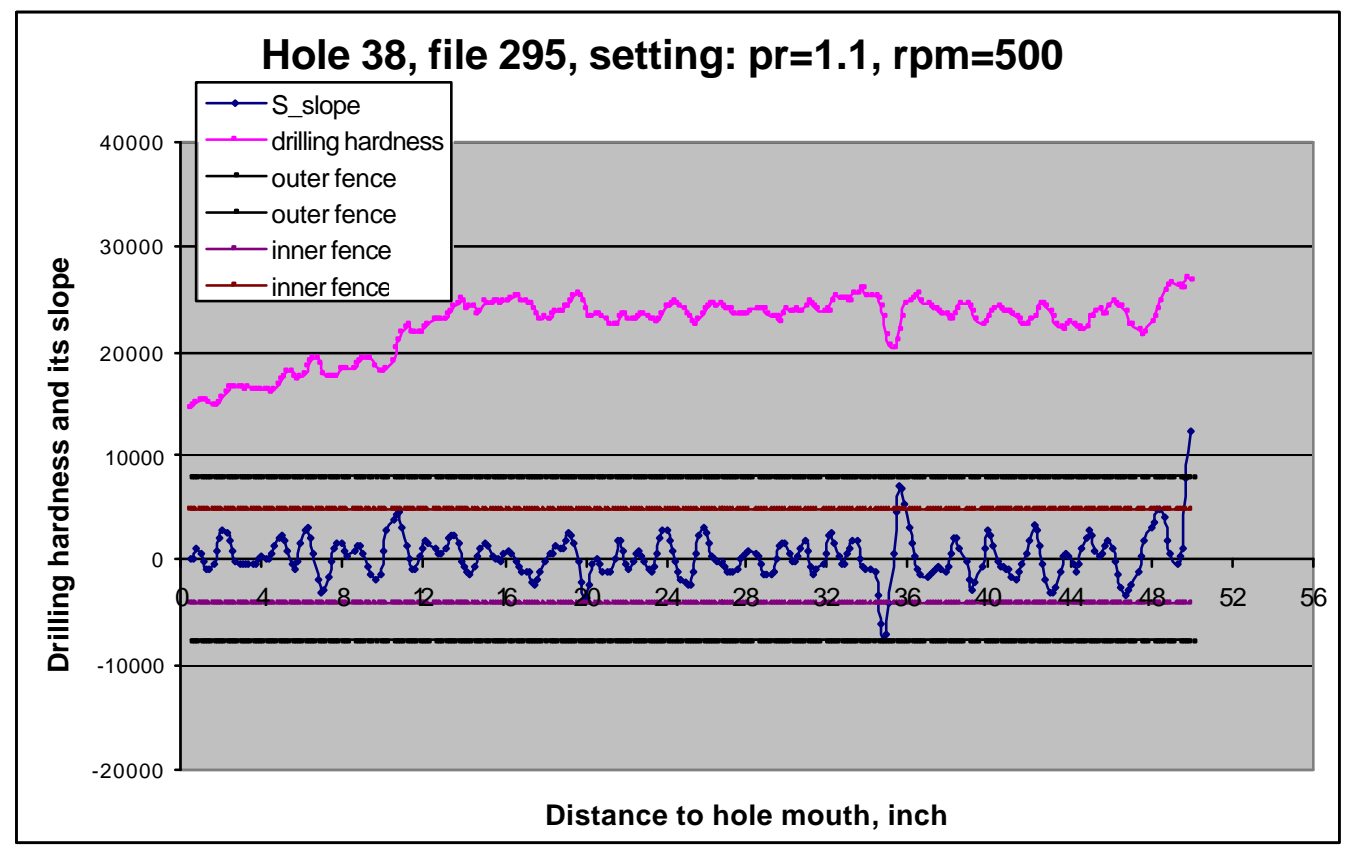

Figure II- 44 Drilling hardness and slope for Hole 328, Solid Block

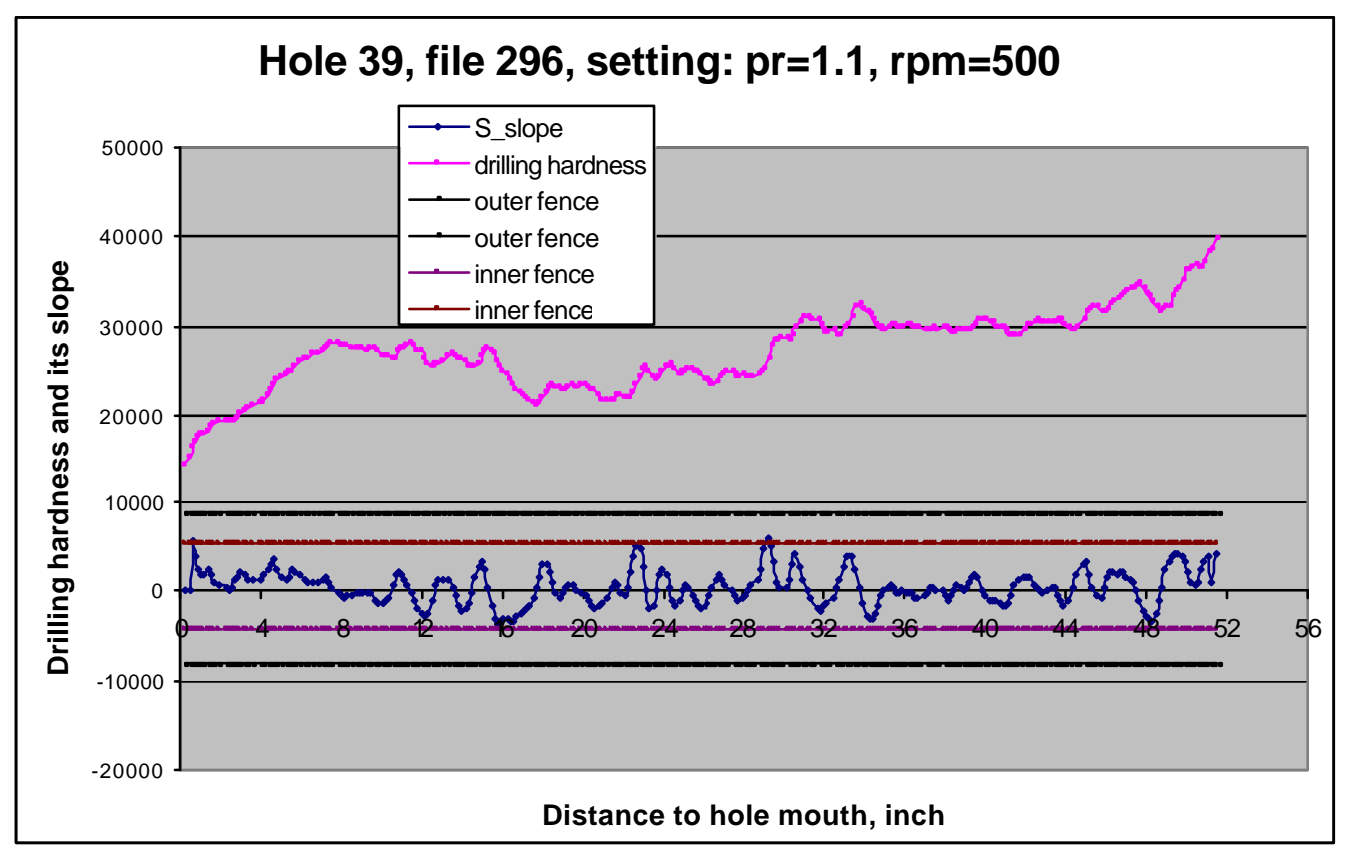

Figure II- 45 Drilling hardness and slope for Hole 39, Solid Block 


\section{Appendix III}

\section{Appendix III}

\section{Calculation Procedure for Rock Type Classification/Prediction}

In this section, the detailed calculation procedure of rock type classification using PDA is presented.

(1) Scaling standardization

Suppose that there are 3 types of rock (A, B, and C), $\mathrm{n}_{1}$ data sets are measured in rock $\mathrm{A}, \mathrm{m}_{2}$ data sets in rock $\mathrm{B}$, and $\mathrm{n}_{3}$ data sets in rock $\mathrm{C}$. Each data set can be considered as a vector with three parameters $x_{1}, x_{2}$, and $x_{3}$ (Figure III-1). In this case, there are three group data, $\mathrm{g}_{1}, \mathrm{~g}_{2}$, and $\mathrm{g}_{3}$. 


\section{Appendix III}

$$
\begin{aligned}
& \text { group } 1 \text { (Rock A) } \\
& \left(\mathrm{x}_{11}, \mathrm{x}_{12}, \mathrm{x}_{13}\right) \\
& \mathrm{n}_{1} \text { sets }\left(\mathrm{x}_{21}, \mathrm{x}_{22}, \mathrm{x}_{23}\right) \\
& \vdots \quad \vdots \quad \vdots \\
& \left(\mathrm{x}_{n_{1} 1}, \mathrm{x}_{\mathrm{n}_{1} 2}, \mathrm{x}_{\mathrm{n}_{1} 3}\right) \\
& \text { group } 2 \text { (Rock B) } \\
& \mathrm{n}_{2} \text { sets }\left(\mathrm{x}_{11}, \mathrm{x}_{12}, \mathrm{x}_{13}\right) \\
& \left(\mathrm{x}_{21}, \mathrm{x}_{22}, \mathrm{x}_{23}\right) \\
& \vdots \quad \vdots \quad \vdots \\
& \left(\mathrm{x}_{n_{2} 1}, \mathrm{x}_{\mathrm{n}_{2} 2}, \mathrm{x}_{\mathrm{n}_{2} 3}\right) \\
& \text { group } 3 \text { (Rock C) } \\
& \mathrm{n}_{8} \text { sets }\left(\mathrm{x}_{11}, \mathrm{x}_{12}, \mathrm{x}_{13}\right) \\
& \left(\mathrm{x}_{21}, \mathrm{x}_{22}, \mathrm{x}_{23}\right) \\
& \vdots \quad \vdots \quad \vdots \\
& \left(\mathrm{x}_{n_{3} 1}, \mathrm{x}_{\mathrm{n}_{3} 2}, \mathrm{x}_{\mathrm{n}_{3} 3}\right)
\end{aligned}
$$

Figure III-1 Group data of rocks A, B, and C

The algorithm of the scaling standardization is shown as (for instance, for group 1):

$$
X_{i k}^{\prime}=\left(X_{i k}-\text { mean of variable } X_{\mathrm{k}}\right) / \sigma
$$

where $X_{\mathrm{ik}}{ }^{\prime}$ - the standardized value of the $i_{\mathrm{th}}$ data of variable $X_{\mathrm{k}}$ of group $1, \mathrm{k}=1,2,3$, i

$$
=1,2,3 \ldots, \mathrm{n}_{1}
$$




\section{Appendix III}

$X_{\mathrm{ik}}$ - the original value of the $\mathrm{i}_{\mathrm{h}}$ data of variable $\mathrm{X}_{\mathrm{k}}$ of group 1

$\sigma$ - the sample standard deviation of variable $X_{k}$ of group 1

After this step, all the data in groups $\mathrm{g}_{2}, \mathrm{~g}_{2}$, and $\mathrm{g}_{3}$ are standardized and transformed into $\mathrm{g}_{\mathrm{s} 1}, \mathrm{~g}_{\mathrm{s} 2}$, and $\mathrm{g}_{\mathrm{s} 3}$.

(2) Determination of the covariance matrix of each group

For instance, the covariance matrix of $g_{s}$ is given by

$$
S_{1}=\left[\begin{array}{lll}
s_{1}^{2} & \mathrm{~s}_{12} & \mathrm{~s}_{13} \\
s_{12} & \mathrm{~s}_{2}^{2} & \mathrm{~s}_{23} \\
\vdots & \vdots & \vdots \\
\mathrm{s}_{\mathrm{n} 1} & \mathrm{~s}_{\mathrm{n} 2} & \mathrm{~s}_{\mathrm{n} 3}^{2}
\end{array}\right]=\left[s_{j k}\right]
$$

where the element $S_{j k}$ is the variance of two variables $\chi_{j}$ and $\chi_{k}(j, k=1,2,3)$, which is determined by

$\operatorname{cov}\left(x_{j}, x_{k}\right)=s_{j k}=\frac{\sum_{i}\left(x_{i j}-\overline{x_{j}}\right)\left(x_{i k}-\overline{x_{k}}\right)}{n_{1}-1}$

where $\mathrm{i}$ - the $\mathrm{i}_{\text {th }}$ data set

$\mathrm{n}_{1}$ - the total number of data sets of the group 1 (rock A)

$\overline{x_{j}}$ - the mean value of parameter $\mathrm{X}_{\mathrm{j}}, \mathrm{j}=1,2,3$

After this step, the covariance matrices $\left(\mathrm{S}_{1}, \mathrm{~S}_{2}, \mathrm{~S}_{3}\right)$ of the three group data $\mathrm{g}_{\mathrm{s}}, \mathrm{g}_{\mathrm{s} 2}$, and $\mathrm{g}_{\mathrm{s} 3}$ are obtained.

(3) Calculation of mahalanobis distance $D_{u m}$

The mahalanobis distance is calculated by 


\section{Appendix III}

$$
D_{u m}=\left[\left(X_{u}-\mu_{m}\right)^{\prime} S_{m}^{-1}\left(X_{u}-\mu_{m}\right)\right]^{\frac{1}{2}}
$$

where $X_{u}$ - the measured data set (vector)

$\mu_{\mathrm{m}}$ - the centroid or mean vector of group $\mathrm{m}(\mathrm{m}=1,2,3$, i.e., rock type $\mathrm{A}, \mathrm{B}, \mathrm{C})$

$\mathrm{S}_{\mathrm{m}}-$ the covariance matrix of group $\mathrm{m}$

After this step, the mahalanobis distances $\left(\mathrm{D}_{\mathrm{u} 1}, \mathrm{D}_{\mathrm{u} 2}\right.$, Du3 $)$ between the measure data $\mathrm{Xu}$ and the the centroids of the three group are obtained.

(4) Posterior probability $\mathrm{P}_{\mathrm{m}}$

The posterior probability $\mathrm{Pm}$ of the measure data set $\mathrm{Xu}$ belonging to group $\mathrm{m}$ is calculated by

$$
P\left(m \mid X_{u}\right)=\frac{\left|S_{m}\right|^{-\frac{1}{2}} \exp \left(-\frac{1}{2} D_{u m}^{2}\right)}{\sum_{m^{\prime}=1}^{g}\left|S_{m^{\prime}}\right|^{-\frac{1}{2}} \exp \left(-\frac{1}{2} D_{u m}^{2}\right)}
$$

Since $D_{u m}$ and $S_{m}$ have been determined in step (3) and (2), the posterior probabilities $\left(\mathrm{P}_{1}, \mathrm{P}_{2}, \mathrm{P}_{3}\right)$ of $\mathrm{Xu}$ belonging to the three groups (rock types) can be obtained. By comparing $\mathrm{P}_{1}, \mathrm{P}_{2}, \mathrm{P}_{3}$, if $\mathrm{P}_{1}$ is the maximum among the three probabilities, it means this measured data $X_{u}$ belongs to group 1, i.e. rock $A$. if $P_{2}$ is the maximum among the three probabilities, it means this measured data $X_{\mathrm{u}}$ belongs to group 2, i.e. rock B, and so on. 
Appendix IV

Appendix IV

\section{Analysis Results from Mine B}

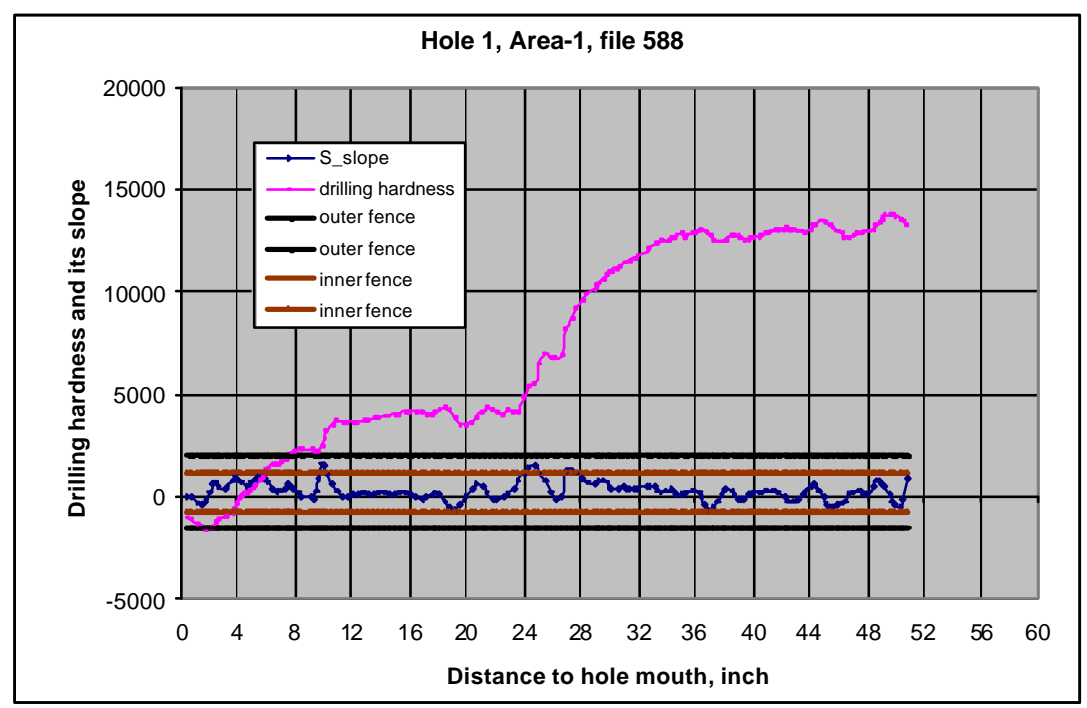

Figure IV- 1 Drilling hardness and slope, Hole 1, Area-1, Mine B 


\section{Appendix IV}

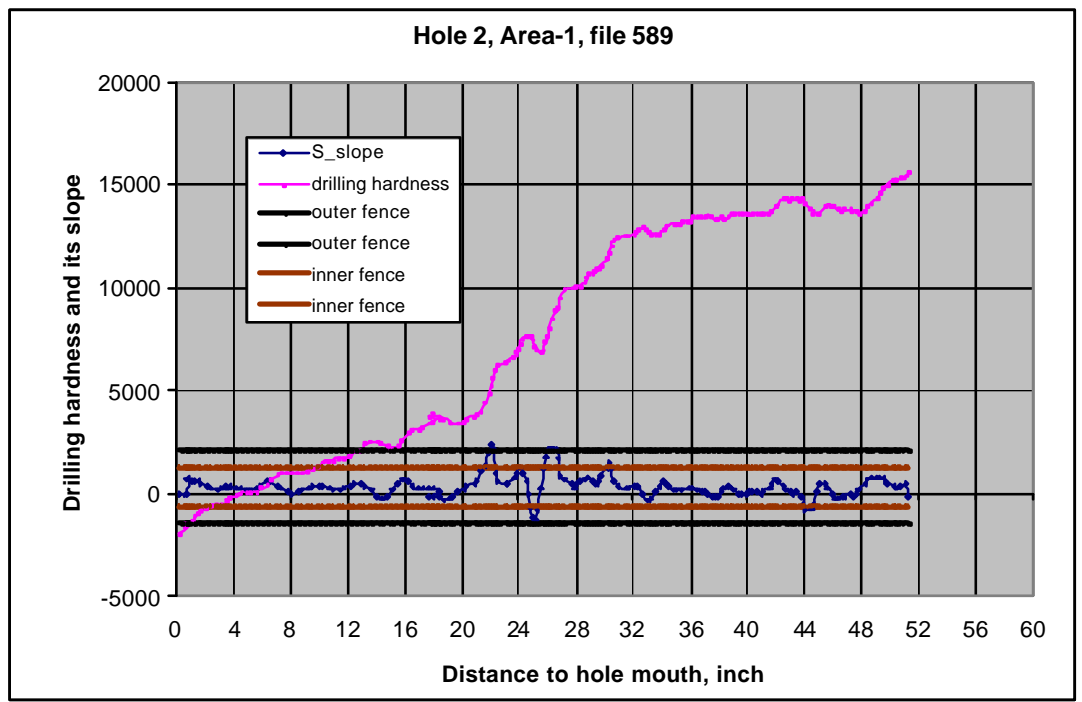

Figure IV- 2 Drilling hardness and slope, Hole 2, Area-1, Mine B

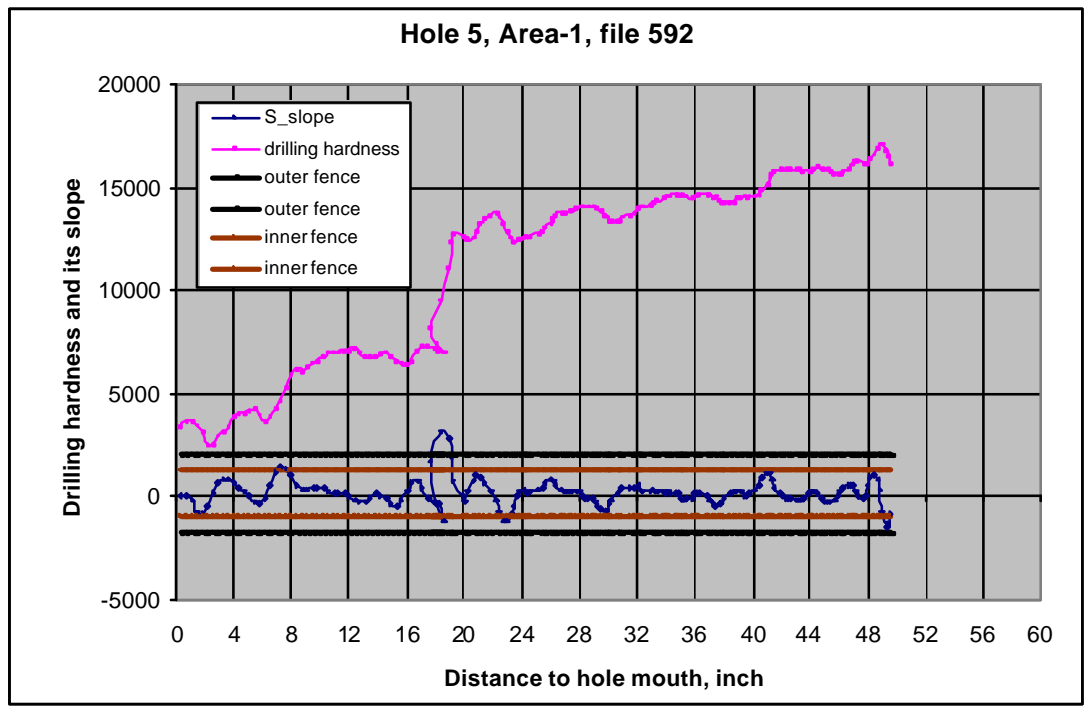

Figure IV- 3 Drilling hardness and slope, Hole 5, Area-1, Mine B 


\section{Appendix IV}

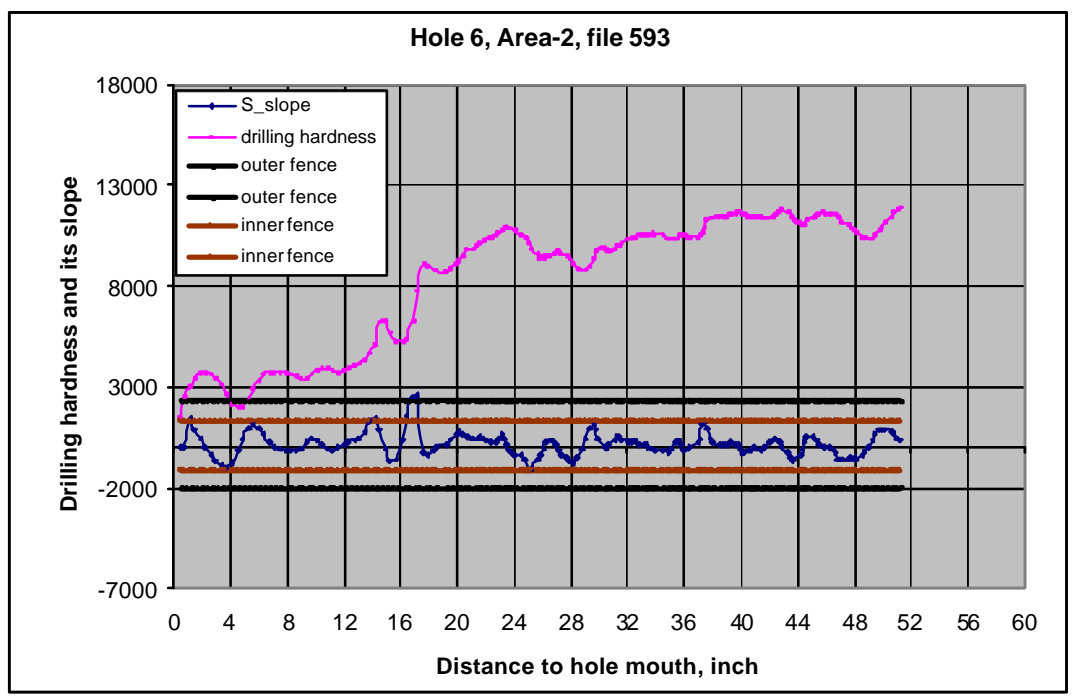

Figure IV- 4 Drilling hardness and slope, Hole 6, Area-2, Mine B

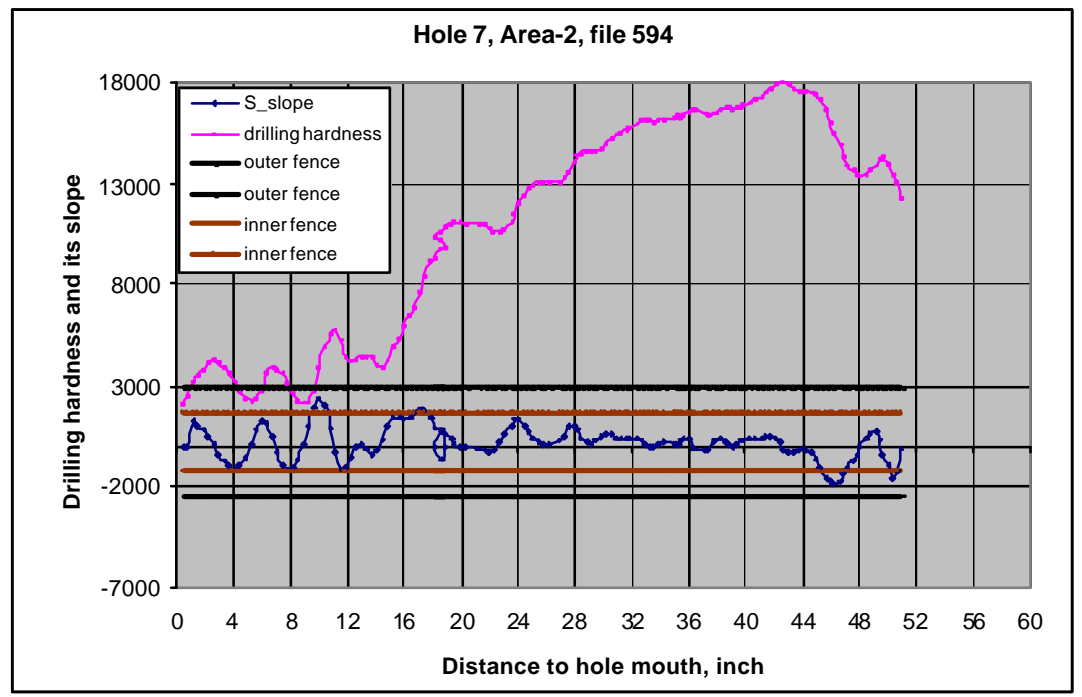

Figure IV- 5 Drilling hardness and slope, Hole 7, Area-2, Mine B 


\section{Appendix IV}

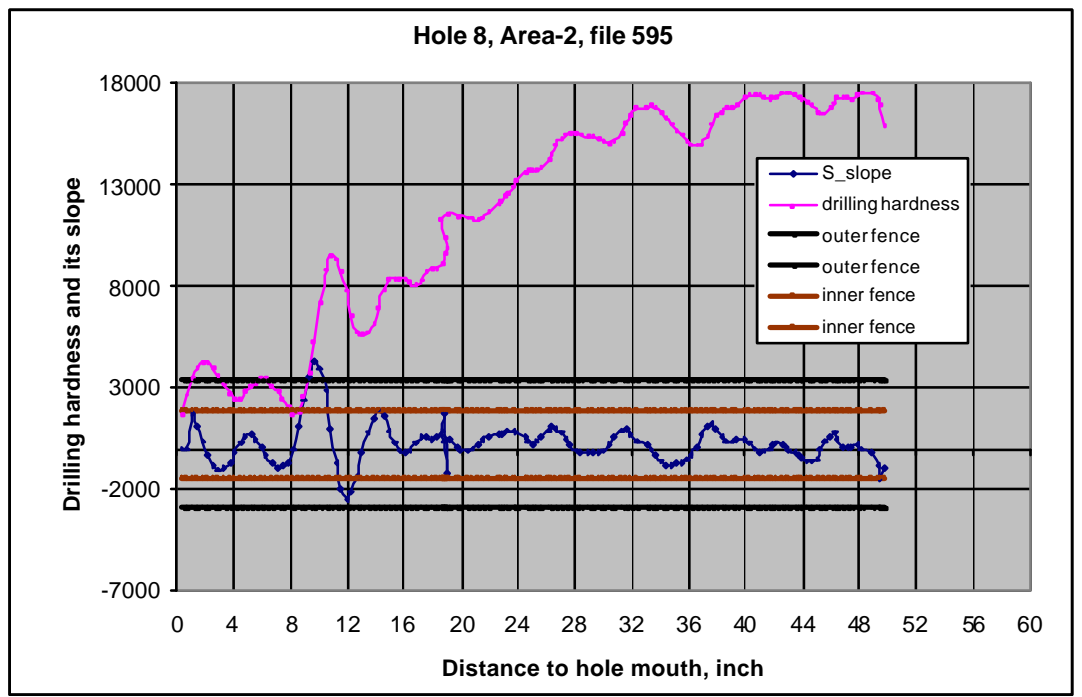

Figure IV- 6 Drilling hardness and slope, Hole 8, Area-2, Mine B

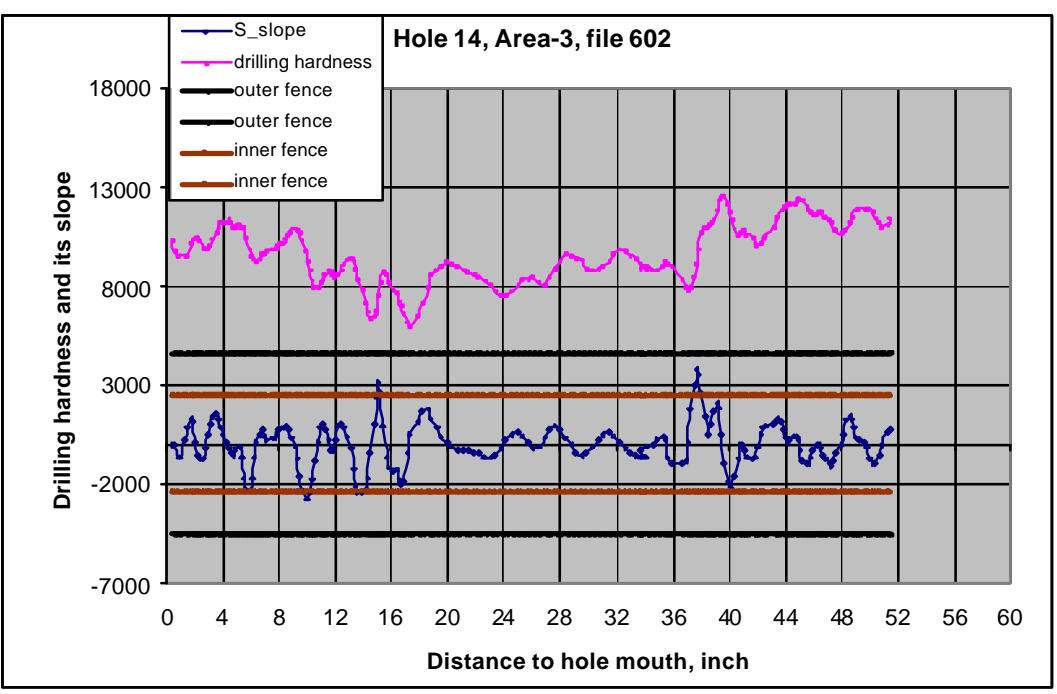

Figure IV- 7 Drilling hardness and slope, Hole 14, Area-3, Mine B 


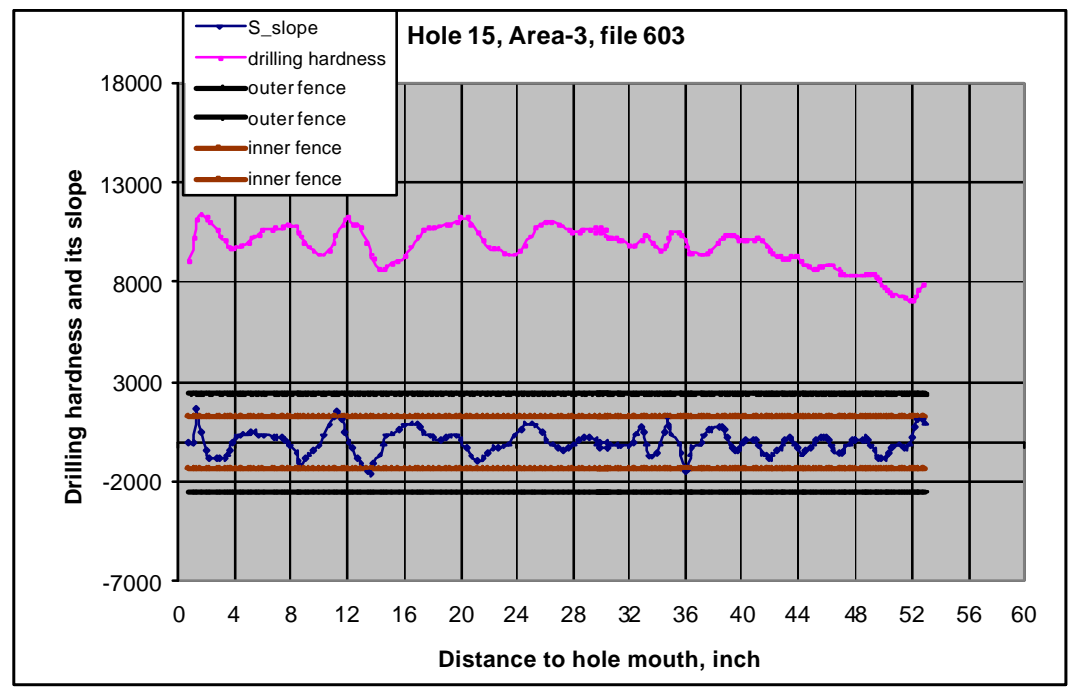

Figure IV- 8 Drilling hardness and slope, Hole 15, Area-3, Mine B

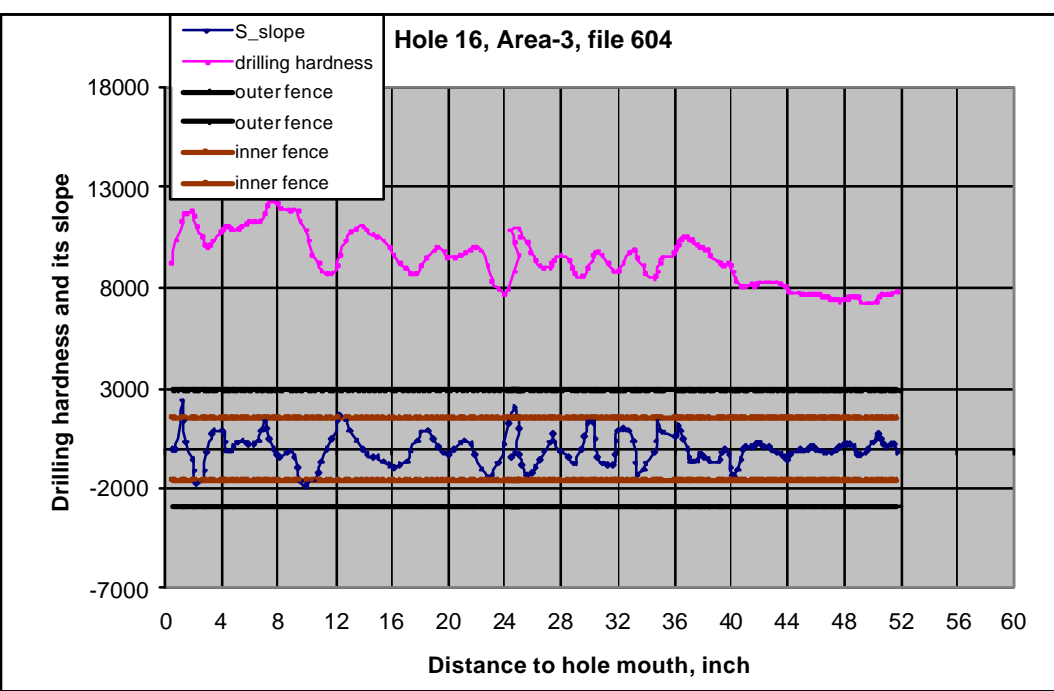

Figure IV- 9 Drilling hardness and slope, Hole 16, Area-3, Mine B 


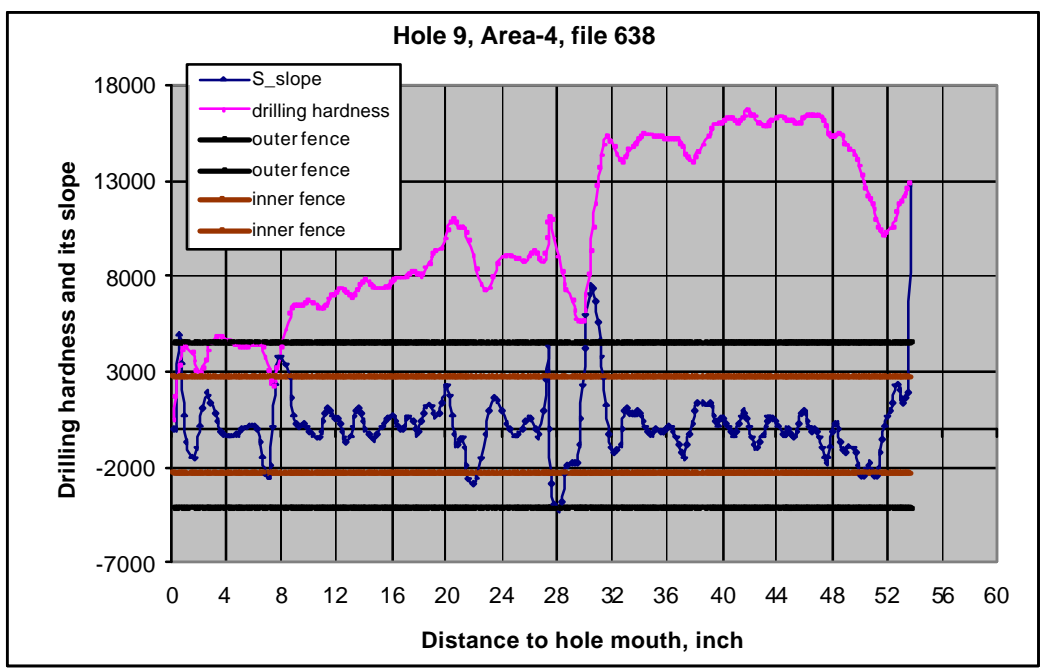

Figure IV- 10 Drilling hardness and slope, Hole 9, Area-4, Mine B

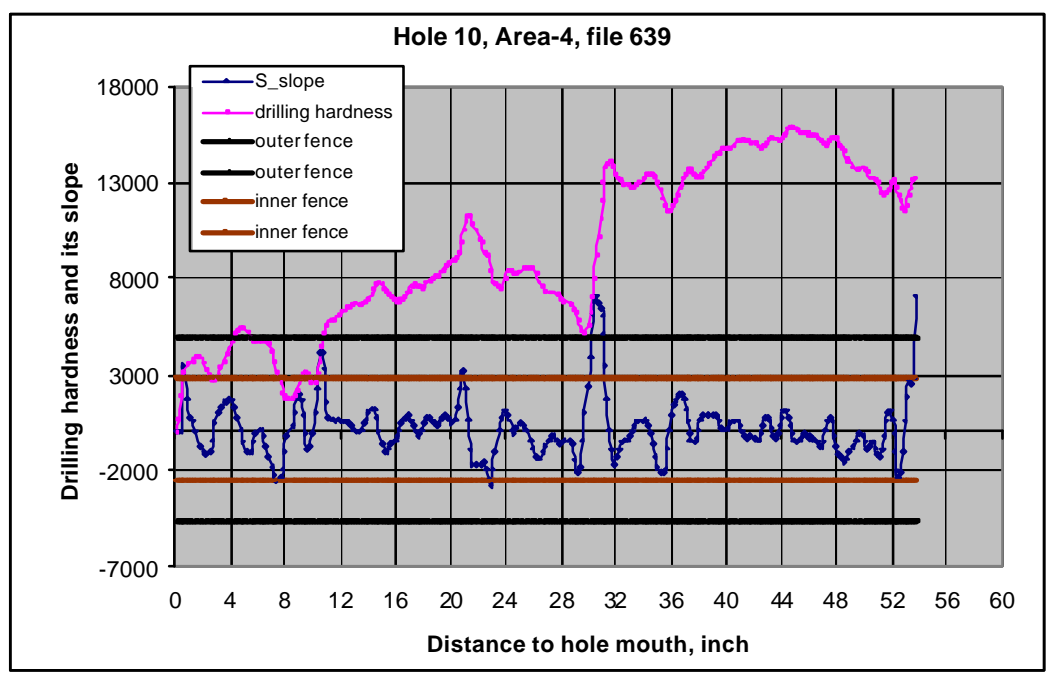

Figure IV- 11 Drilling hardness and slope, Hole 10, Area-4, Mine B 


\section{Appendix IV}

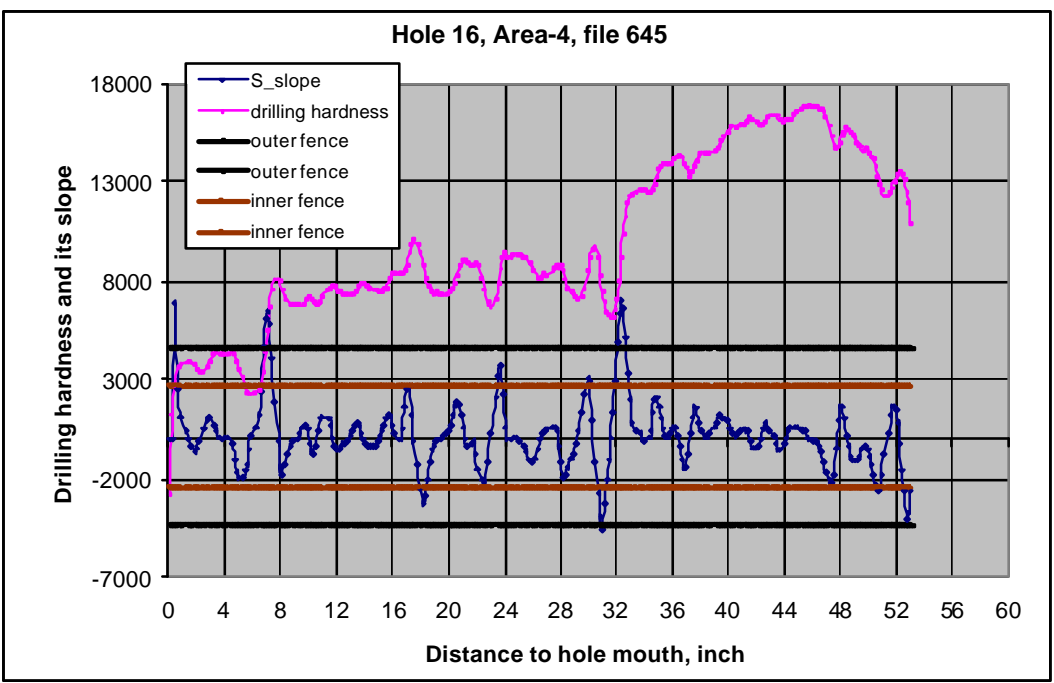

Figure IV- 12 Drilling hardness and slope, Hole 16, Area-4, Mine B

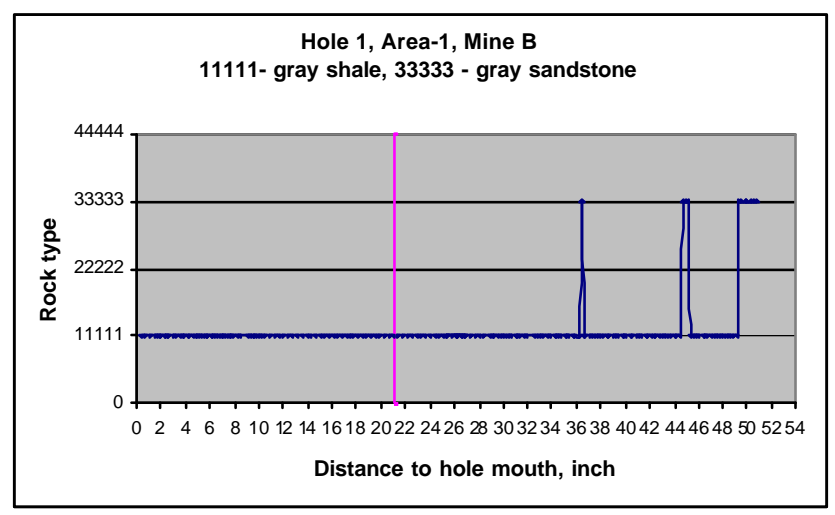

Figure IV- 13 Rock type classification result, Hole 1, Area-1, Mine B (group data obtained from Area-4)

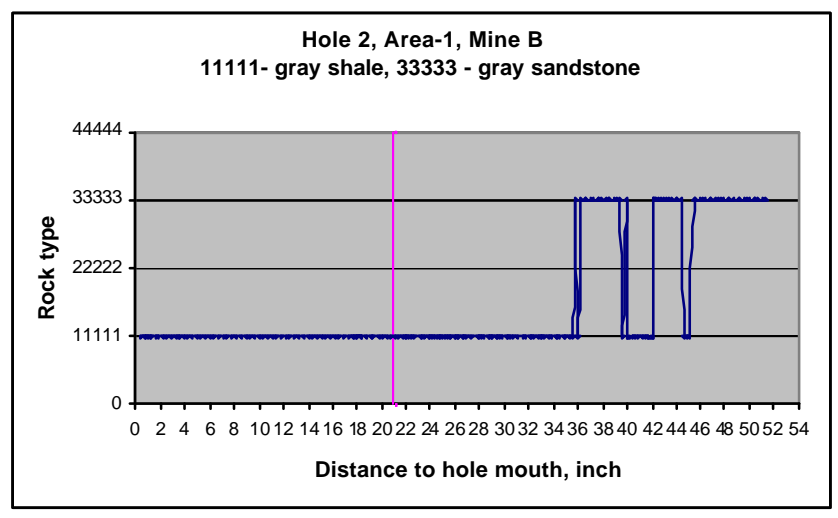

Figure IV- 14 Rock type classification result, Hole 2, Area-1, Mine B (group data obtained from Area-4) 


\section{Appendix IV}

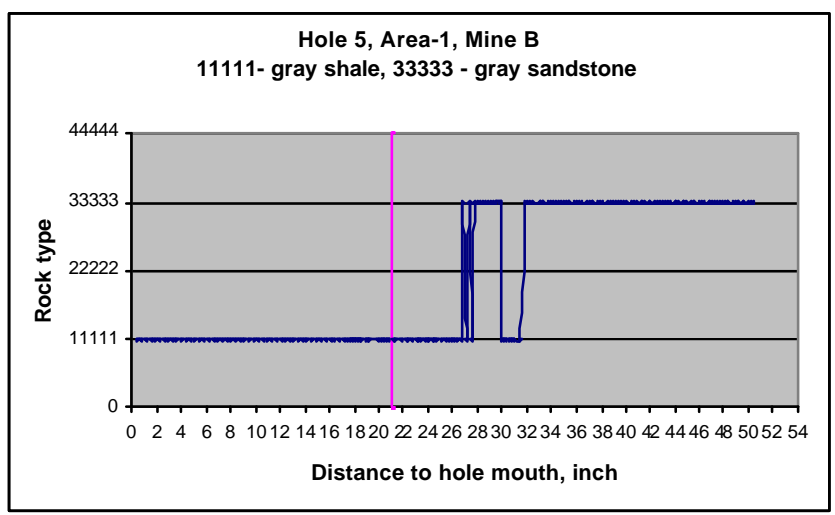

Figure IV- 15 Rock type classification result, Hole 5, Area-1, Mine B (group data obtained from Area-4)

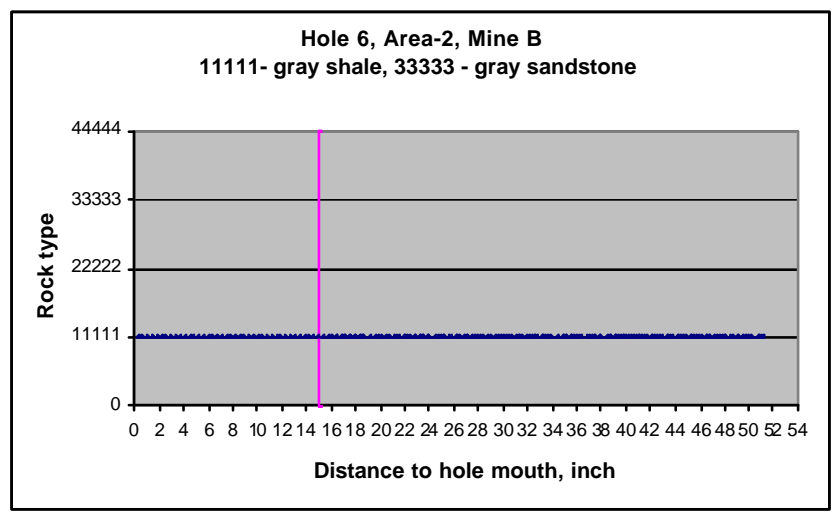

Figure IV- 16 Rock type classification result, Hole 6, Area-2, Mine B (group data obtained from Area-4)

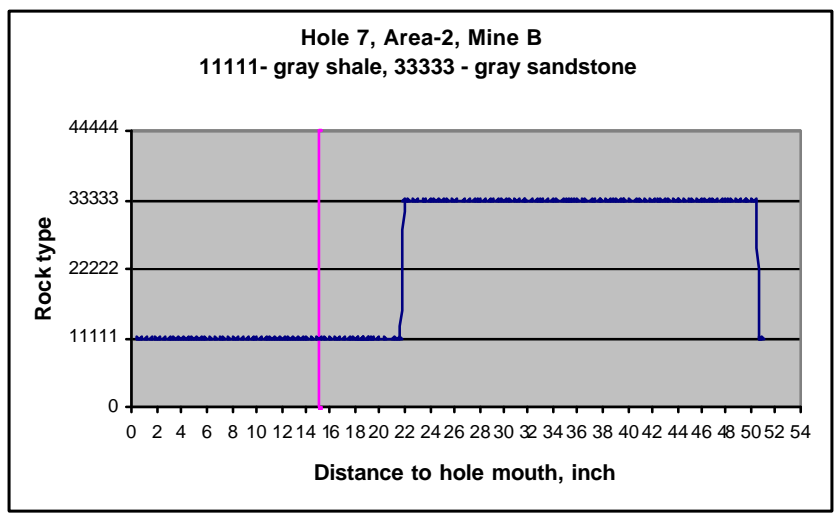

Figure IV- 17 Rock type classification result, Hole 7, Area-2, Mine B (group data obtained from Area-4) 


\section{Appendix IV}

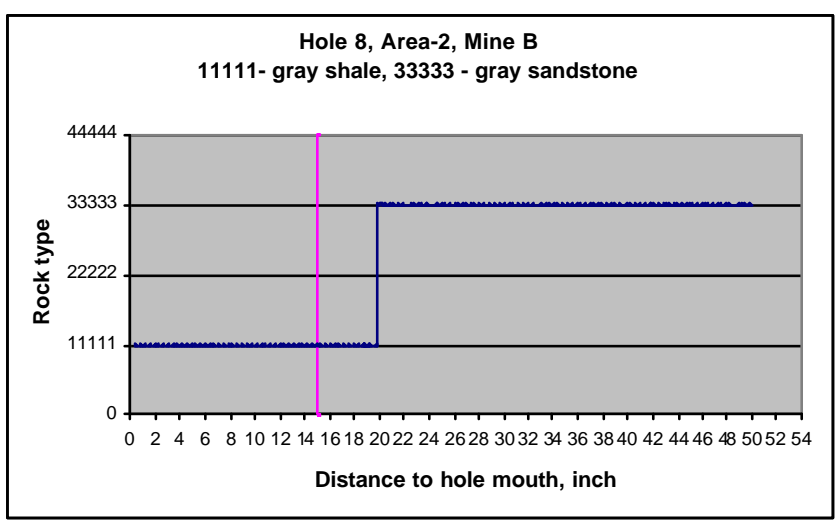

Figure IV- 18 Rock type classification result, Hole 8, Area-2, Mine B (group data obtained from Area-4)

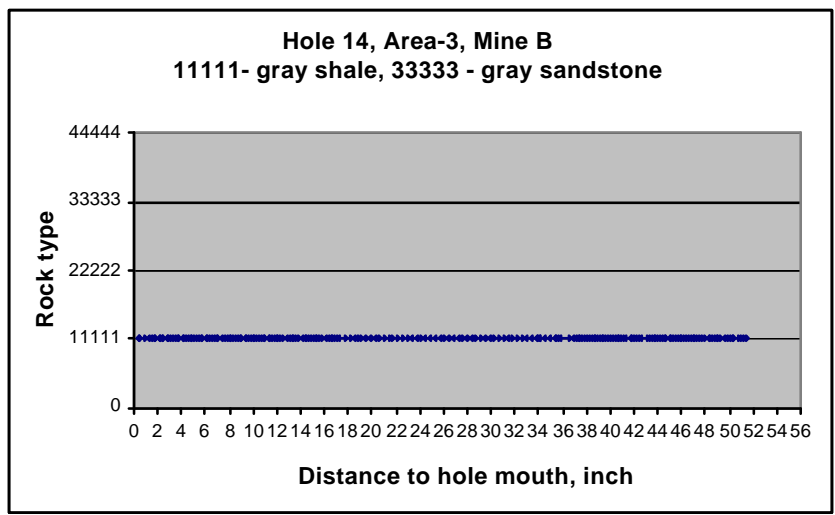

Figure IV- 19 Rock type classification result, Hole 14, Area-3, Mine B (group data obtained from Area-4)

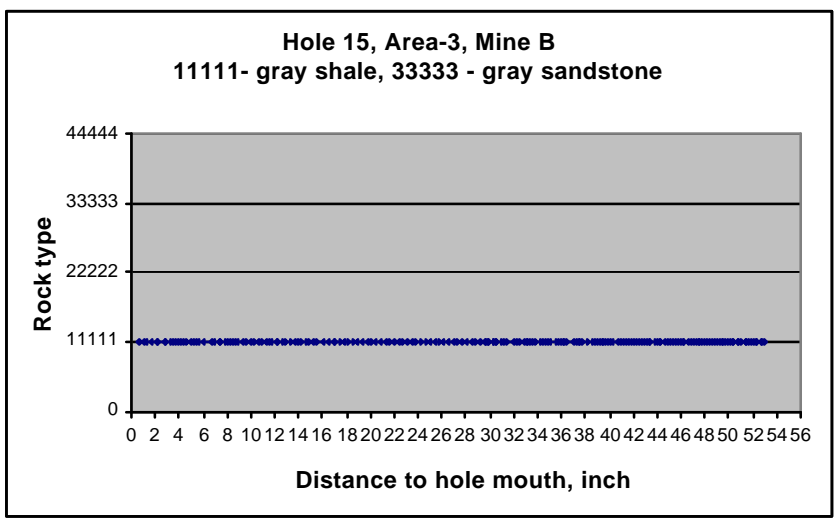

Figure IV- 20 Rock type classification result, Hole 15, Area-3, Mine B (group data obtained from Area-4) 


\section{Appendix IV}

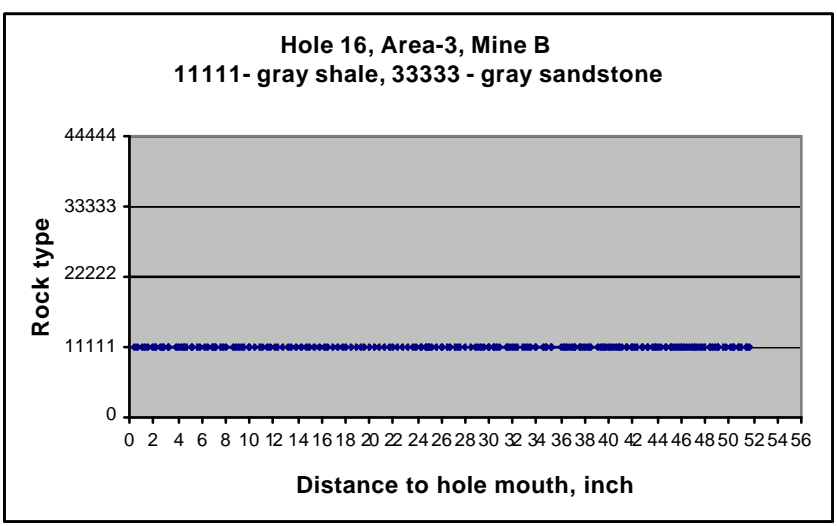

Figure IV- 21 Rock type classification result, Hole 16, Area-3, Mine B (group data obtained from Area-4)

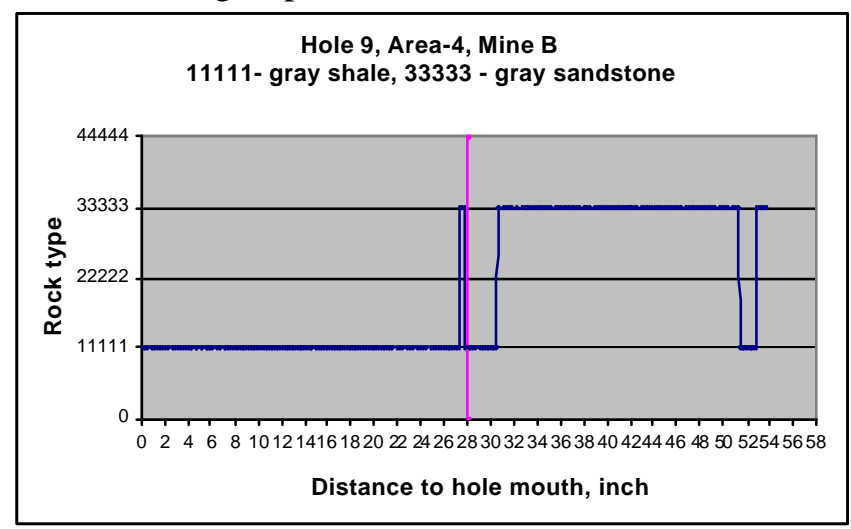

Figure IV- 22 Rock type classification result, Hole 9, Area-4, Mine B (group data obtained from Area-4)

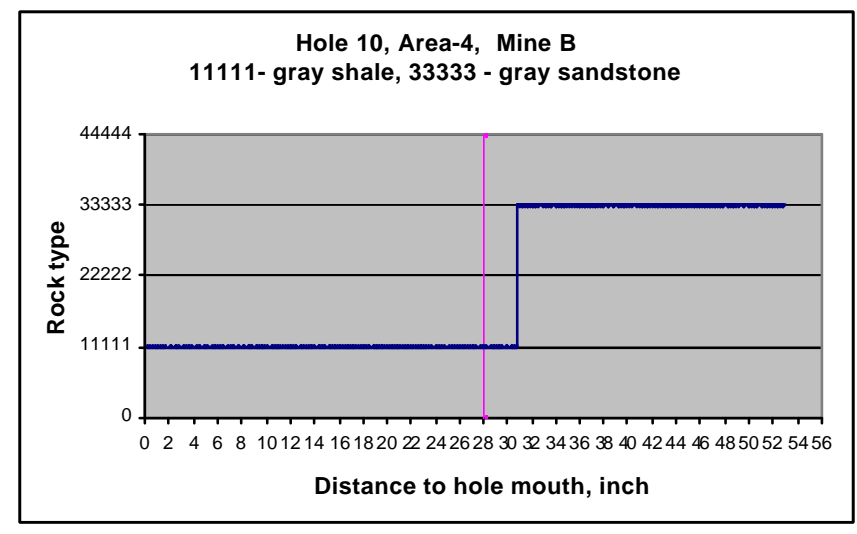

Figure IV- 23 Rock type classification result, Hole 10, Area-4, Mine B (group data obtained from Area-4) 


\section{Appendix IV}

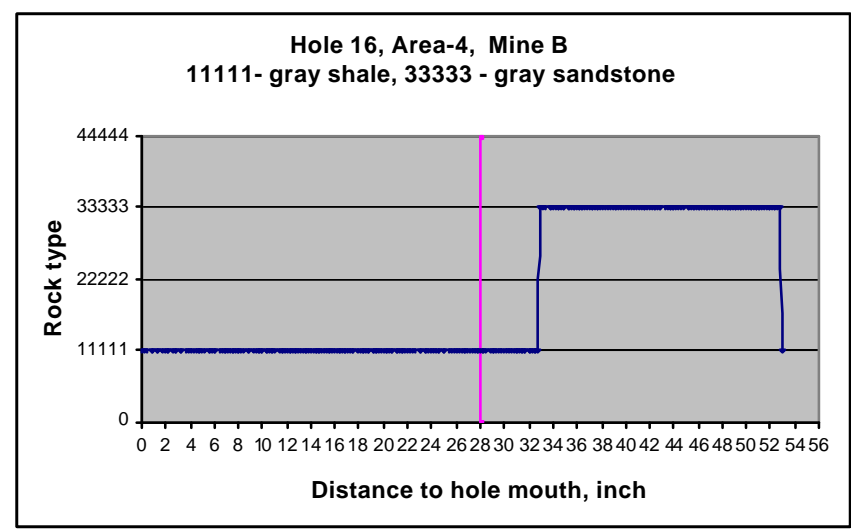

Figure IV- 24 Rock type classification result, Hole 16, Area-4, Mine B

(group data obtained from Area-4)

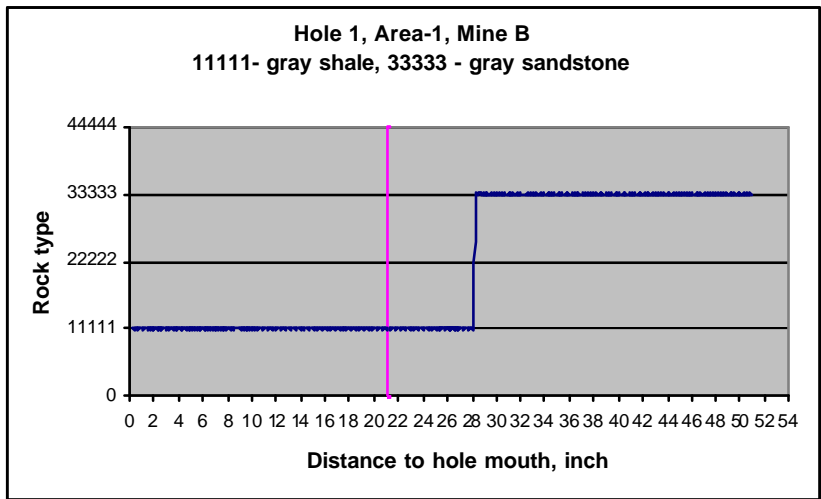

Figure IV- 25 Rock type classification result, Hole 1, Area-1, Mine B (group data obtained from Area-1, 2, 3, and 4)

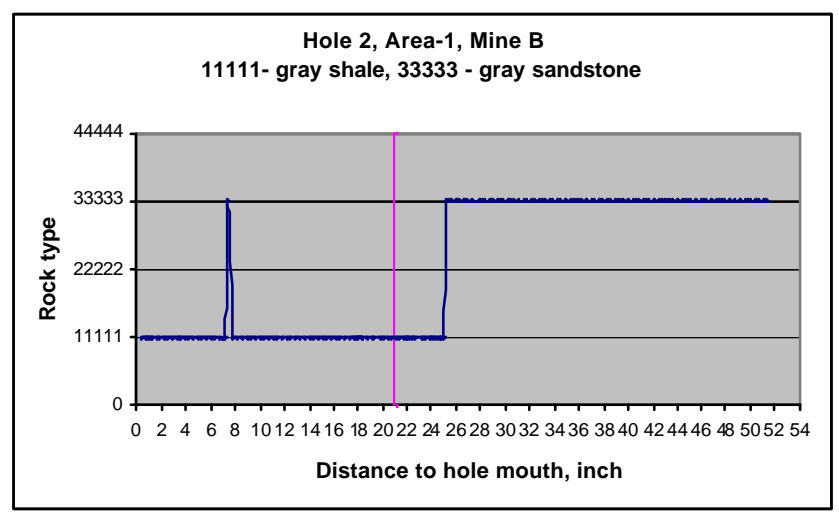

Figure IV- 26 Rock type classification result, Hole 2, Area-1, Mine B (group data obtained from Area-1, 2, 3, and 4) 


\section{Appendix IV}

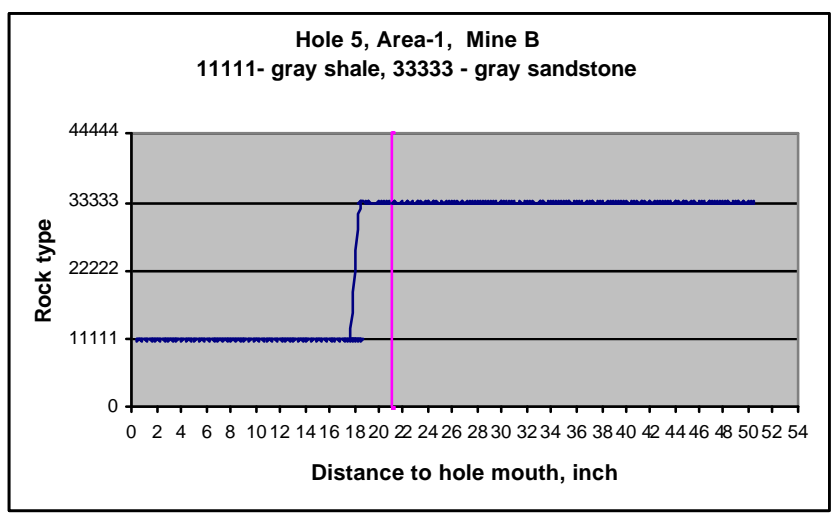

Figure IV- 27 Rock type classification result, Hole 5, Area-1, Mine B (group data obtained from Area-1, 2, 3, and 4)

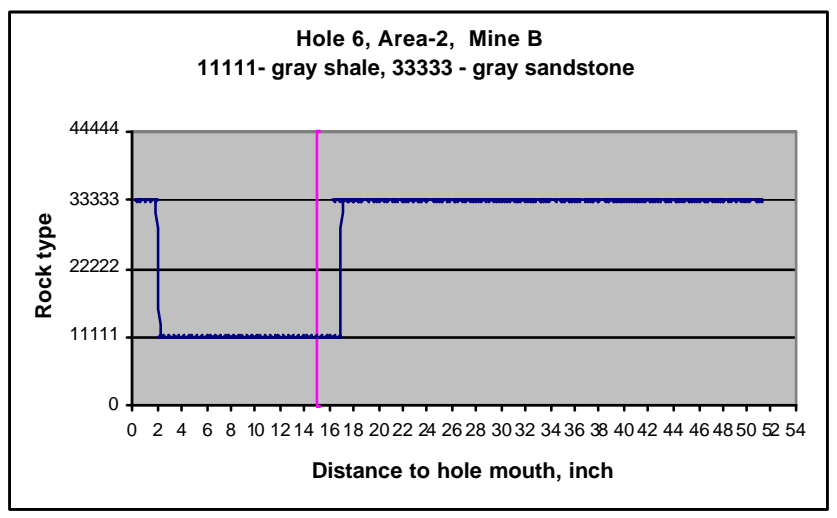

Figure IV- 28 Rock type classification result, Hole 6, Area-2, Mine B (group data obtained from Area-1, 2, 3, and 4)

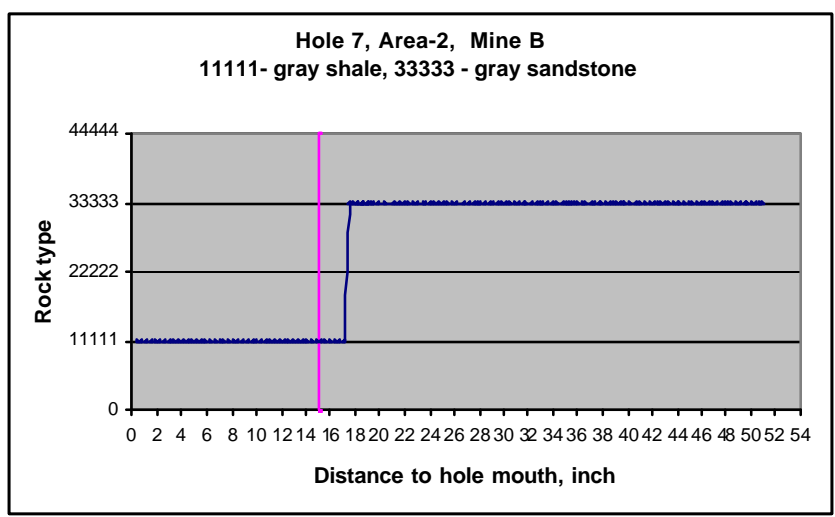

Figure IV- 29 Rock type classification result, Hole 7, Area-2, Mine B (group data obtained from Area-1, 2, 3, and 4) 


\section{Appendix IV}

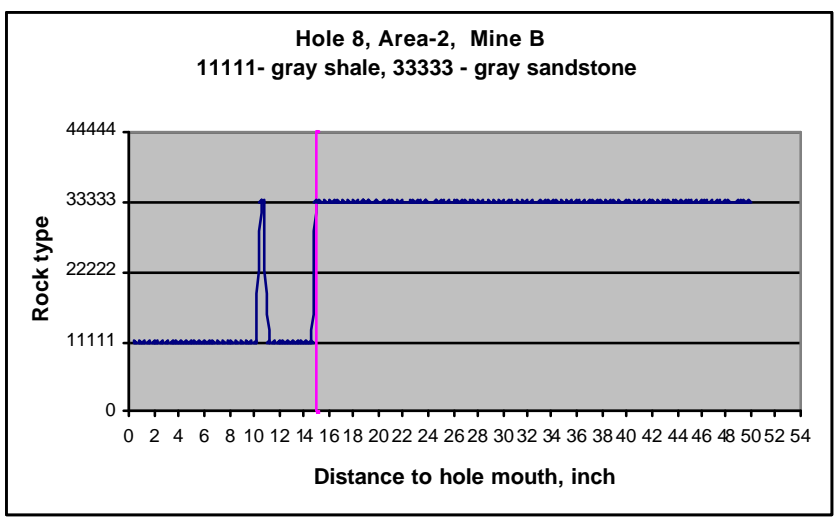

Figure IV- 30 Rock type classification result, Hole 8, Area-2, Mine B

(group data obtained from Area-1, 2, 3, and 4)

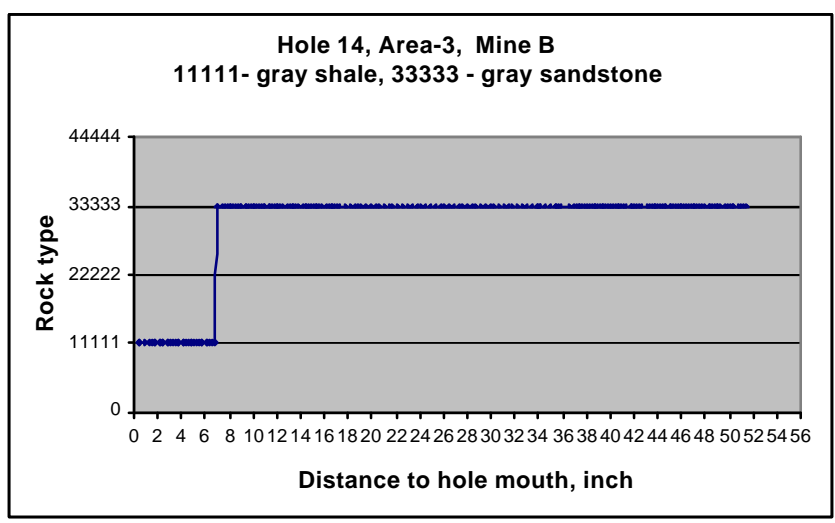

Figure IV- 31 Rock type classification result, Hole 14, Area-3, Mine B (group data obtained from Area-1, 2, 3, and 4)

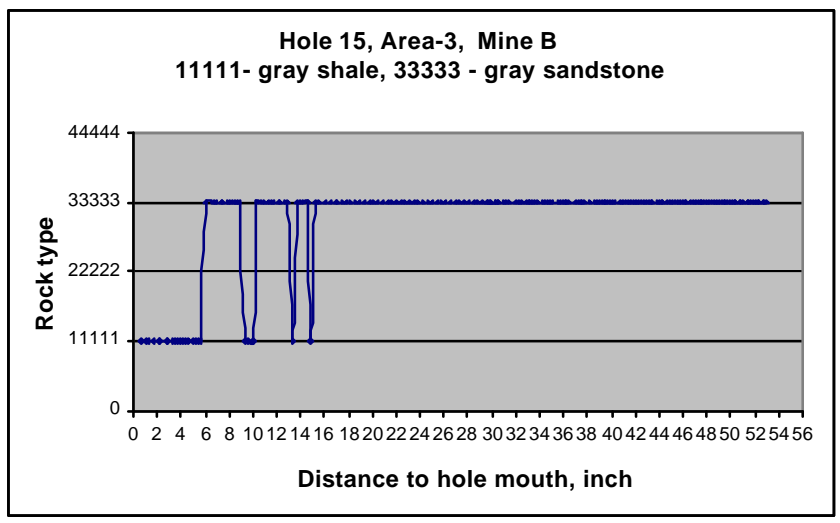

Figure IV- 32 Rock type classification result, Hole 15, Area-3, Mine B (group data obtained from Area-1, 2, 3, and 4) 


\section{Appendix IV}

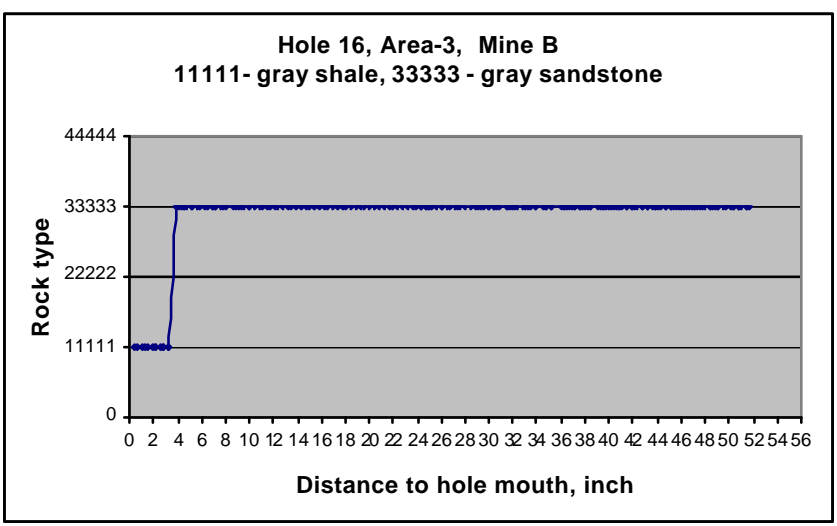

Figure IV- 33 Rock type classification result, Hole 16, Area-3, Mine B (group data obtained from Area-1, 2, 3, and 4)

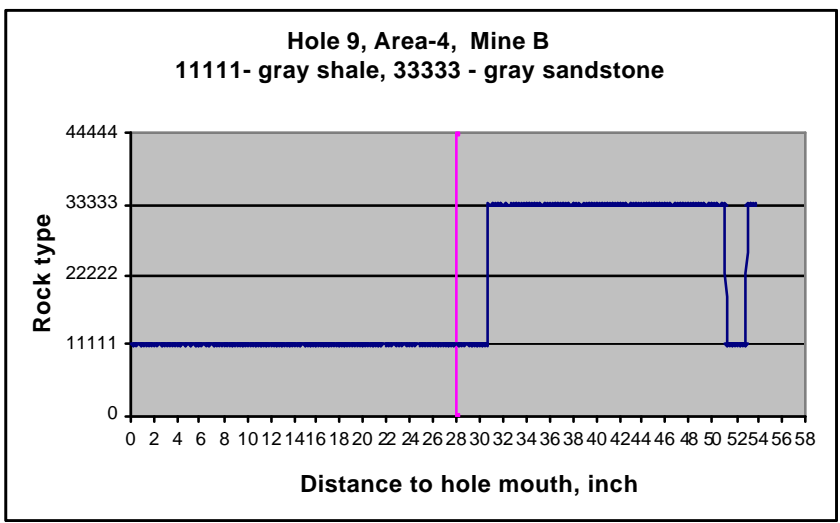

Figure IV- 34 Rock type classification result, Hole 9, Area-4, Mine B (group data obtained from Area-1, 2, 3, and 4)

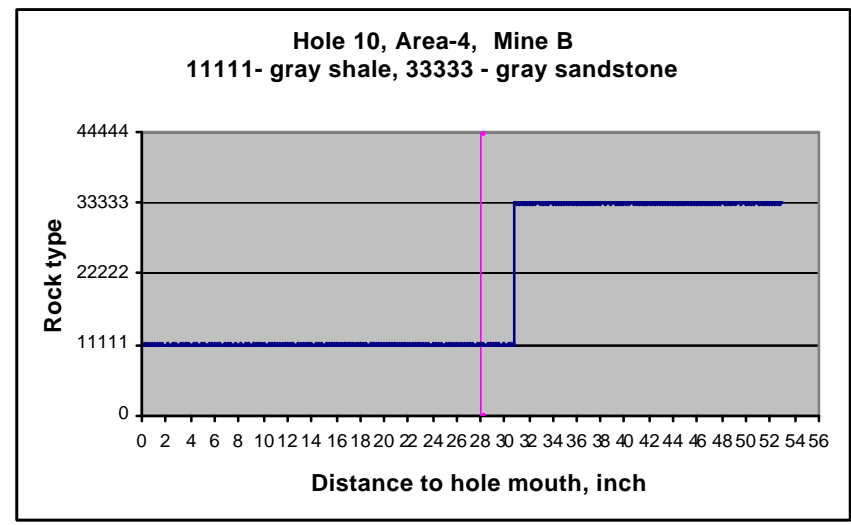

Figure IV- 35 Rock type classification result, Hole 10, Area-4, Mine B (group data obtained from Area-1, 2, 3, and 4) 


\section{Appendix IV}

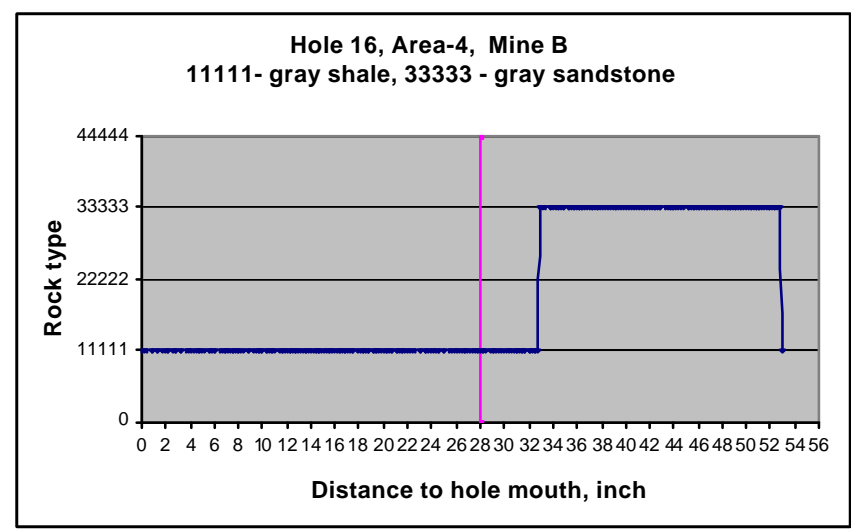

Figure IV- 36 Rock type classification result, Hole 16, Area-4, Mine B

(group data obtained from Area-1, 2, 3, and 4)

Table IV- 34 Rock type classification/prediction results from Mine B (Area-1, 2, 3 and 4)

\begin{tabular}{|c|c|c|c|c|}
\hline \multicolumn{2}{|c|}{ Drilled holes } & $\begin{array}{c}\text { Recorded numbers of } \\
\text { points }\end{array}$ & $\begin{array}{c}\text { Training data obtained } \\
\text { Area Area-4 }\end{array}$ & $\begin{array}{l}\text { Training data obtained } \\
\text { from Area-1,2, } 3 \text { and } 4\end{array}$ \\
\hline \multirow{6}{*}{$\begin{array}{l}\text { Hole 1, } \\
\text { Area-1 }\end{array}$} & \multirow{3}{*}{ Gray shale } & Number of original points & 76 & 76 \\
\hline & & Number of predicted points & 76 & 76 \\
\hline & & Hit rate $(\%)$ & 100 & 100 \\
\hline & \multirow{3}{*}{ Gray sandstone } & Number of original points & 118 & 118 \\
\hline & & Number of predicted points & 11 & 93 \\
\hline & & Hit rate $(\%)$ & 9.32 & 78.81 \\
\hline \multirow{6}{*}{$\begin{array}{l}\text { Hole 2, } \\
\text { Area-1 }\end{array}$} & \multirow{3}{*}{ Gray shale } & Number of original points & 65 & 65 \\
\hline & & Number of predicted points & 65 & 64 \\
\hline & & Hit rate $(\%)$ & 100 & 98.46 \\
\hline & \multirow{3}{*}{ Gray sandstone } & Number of original points & 129 & 129 \\
\hline & & Number of predicted points & 50 & 110 \\
\hline & & Hit rate $(\%)$ & 38.76 & 85.27 \\
\hline \multirow{6}{*}{$\begin{array}{l}\text { Hole 5, } \\
\text { Area-1 }\end{array}$} & \multirow{3}{*}{ Gray shale } & Number of original points & 62 & 62 \\
\hline & & Number of predicted points & 62 & 53 \\
\hline & & Hit rate $(\%)$ & 100 & 85.48 \\
\hline & \multirow{3}{*}{ Gray sandstone } & Number of original points & 126 & 126 \\
\hline & & Number of predicted points & 95 & 126 \\
\hline & & Hit rate $(\%)$ & 75.4 & 100 \\
\hline \multirow{6}{*}{$\begin{array}{l}\text { Hole 6, } \\
\text { Area-2 }\end{array}$} & \multirow{3}{*}{ Gray shale } & Number of original points & 40 & 40 \\
\hline & & Number of predicted points & 40 & 35 \\
\hline & & Hit rate $(\%)$ & 100 & 87.5 \\
\hline & \multirow{3}{*}{ Gray sandstone } & Number of original points & 160 & 160 \\
\hline & & Number of predicted points & 0 & 155 \\
\hline & & Hit rate $(\%)$ & $\mathbf{0}$ & 96.88 \\
\hline
\end{tabular}




\section{Appendix IV}

\begin{tabular}{|c|c|c|c|c|}
\hline \multirow{6}{*}{$\begin{array}{l}\text { Hole 7, } \\
\text { Area-2 }\end{array}$} & \multirow{3}{*}{ Gray shale } & Number of original points & 40 & 40 \\
\hline & & Number of predicted points & 40 & 40 \\
\hline & & Hit rate $(\%)$ & 100 & 100 \\
\hline & \multirow{3}{*}{ Gray sandstone } & Number of original points & 110 & 110 \\
\hline & & Number of predicted points & 87 & 104 \\
\hline & & Hit rate $(\%)$ & 79.09 & 94.55 \\
\hline \multirow{6}{*}{$\begin{array}{l}\text { Hole 8, } \\
\text { Area-2 }\end{array}$} & \multirow{3}{*}{ Gray shale } & Number of original points & 40 & 40 \\
\hline & & Number of predicted points & 40 & 37 \\
\hline & & Hit rate $(\%)$ & 100 & 92.5 \\
\hline & \multirow{3}{*}{ Gray sandstone } & Number of original points & 101 & 101 \\
\hline & & Number of predicted points & 85 & 101 \\
\hline & & Hit rate $(\%)$ & 84.16 & 100 \\
\hline \multirow{6}{*}{$\begin{array}{c}\text { Hole 14, } \\
\text { Area-3 }\end{array}$} & \multirow{3}{*}{ Gray shale } & Number of original points & 0 & 0 \\
\hline & & Number of predicted points & 215 & 34 \\
\hline & & Hit rate $(\%)$ & N/A* & N/A \\
\hline & \multirow{3}{*}{ Gray sandstone } & Number of original points & 215 & 215 \\
\hline & & Number of predicted points & 0 & 181 \\
\hline & & Hit rate $(\%)$ & $\mathbf{0}$ & 84.19 \\
\hline \multirow{6}{*}{$\begin{array}{c}\text { Hole } 15, \\
\text { Area-3 }\end{array}$} & \multirow{3}{*}{ Gray shale } & Number of original points & 0 & 0 \\
\hline & & Number of predicted points & 195 & 24 \\
\hline & & Hit rate $(\%)$ & $\mathbf{N} / \mathbf{A}$ & N/A \\
\hline & \multirow{3}{*}{ Gray sandstone } & Number of original points & 195 & 195 \\
\hline & & Number of predicted points & 0 & 171 \\
\hline & & Hit rate $(\%)$ & $\mathbf{0}$ & 87.69 \\
\hline \multirow{6}{*}{$\begin{array}{c}\text { Hole 16, } \\
\text { Area-3 }\end{array}$} & \multirow{3}{*}{ Gray shale } & Number of original points & 0 & 0 \\
\hline & & Number of predicted points & 198 & 13 \\
\hline & & Hit rate $(\%)$ & $\mathbf{N} / \mathbf{A}$ & N/A \\
\hline & Gray sandstone & Number of original points & 198 & 198 \\
\hline & & Number of predicted points & 0 & 185 \\
\hline & & Hit rate $(\%)$ & $\mathbf{0}$ & 93.43 \\
\hline \multirow{6}{*}{$\begin{array}{l}\text { Hole 9, } \\
\text { Area-4 }\end{array}$} & \multirow{3}{*}{ Gray shale } & Number of original points & 145 & 145 \\
\hline & & Number of predicted points & 142 & 145 \\
\hline & & Hit rate $(\%)$ & 97.93 & 100 \\
\hline & \multirow{3}{*}{ Gray sandstone } & Number of original points & 157 & 157 \\
\hline & & Number of classified & 134 & 130 \\
\hline & & Hit rate $(\%)$ & 85.35 & 82.8 \\
\hline \multirow{6}{*}{$\begin{array}{c}\text { Hole 10, } \\
\text { Area-4 }\end{array}$} & \multirow{3}{*}{ Gray shale } & Number of original points & 147 & 147 \\
\hline & & Number of classified & 147 & 147 \\
\hline & & Hit rate $(\%)$ & 100 & 100 \\
\hline & \multirow{3}{*}{ Gray sandstone } & Number of original points & 159 & 159 \\
\hline & & Number of classified & 145 & 137 \\
\hline & & Hit rate $(\%)$ & 91.19 & 86.16 \\
\hline \multirow{2}{*}{$\begin{array}{l}\text { Hole 16, } \\
\text { Area-4 }\end{array}$} & \multirow[t]{2}{*}{ Gray shale } & Number of original points & 151 & 151 \\
\hline & & Number of classified & 151 & 151 \\
\hline
\end{tabular}




\section{Appendix IV}

\begin{tabular}{|l|c|c|c|c|}
\hline & Hit rate (\%) & $\mathbf{1 0 0}$ & $\mathbf{1 0 0}$ \\
\cline { 2 - 5 } & \multirow{3}{*}{ Gray sandstone } & Number of original points & 149 & 149 \\
\cline { 3 - 5 } & Number of classified & 121 & 120 \\
\cline { 2 - 5 } & Hit rate (\%) & $\mathbf{8 1 . 2 1}$ & $\mathbf{8 0 . 5 4}$ \\
\cline { 2 - 5 } & &
\end{tabular}

Legend: N/A - not available. 